\title{
Findable and reusable?
}

Citation for published version (APA):

Gregory, K. (2021). Findable and reusable? Data discovery practices in research. [Doctoral Thesis, Maastricht University]. Maastricht University. https://doi.org/10.26481/dis.20210302kg

Document status and date:

Published: 01/01/2021

DOI:

$10.26481 /$ dis.20210302kg

Document Version:

Publisher's PDF, also known as Version of record

\section{Please check the document version of this publication:}

- A submitted manuscript is the version of the article upon submission and before peer-review. There can be important differences between the submitted version and the official published version of record.

People interested in the research are advised to contact the author for the final version of the publication, or visit the DOI to the publisher's website.

- The final author version and the galley proof are versions of the publication after peer review.

- The final published version features the final layout of the paper including the volume, issue and page numbers.

Link to publication

\footnotetext{
General rights rights.

- You may freely distribute the URL identifying the publication in the public portal. please follow below link for the End User Agreement:

www.umlib.nl/taverne-license

Take down policy

If you believe that this document breaches copyright please contact us at:

repository@maastrichtuniversity.nl

providing details and we will investigate your claim.
}

Copyright and moral rights for the publications made accessible in the public portal are retained by the authors and/or other copyright owners and it is a condition of accessing publications that users recognise and abide by the legal requirements associated with these

- Users may download and print one copy of any publication from the public portal for the purpose of private study or research.

- You may not further distribute the material or use it for any profit-making activity or commercial gain

If the publication is distributed under the terms of Article $25 \mathrm{fa}$ of the Dutch Copyright Act, indicated by the "Taverne" license above, 


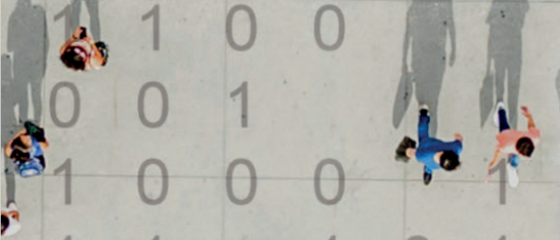

$\begin{array}{llllll}0 & 1 & 0 & 1 & 1\end{array}:$

$1=\begin{array}{llllllllll}1 & 0 & 1 & 1 & 1\end{array}$

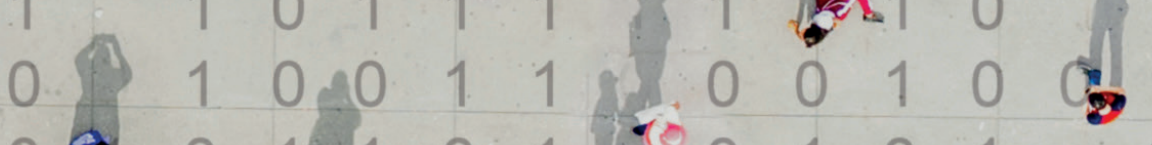

\& $\begin{array}{lllll}0 & 1 / 1 & 0 & 1 & 0 \\ 0 & 1 & 1 & 0\end{array}$

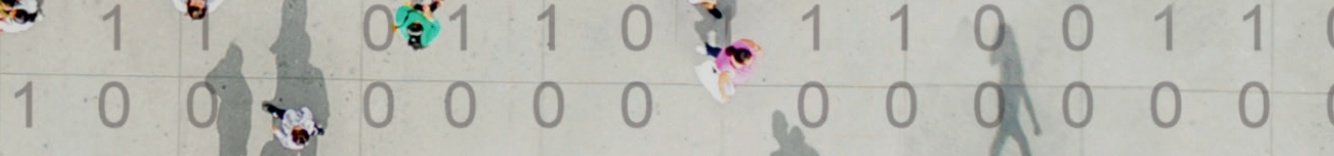

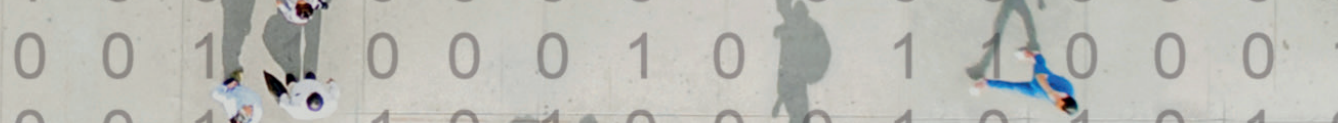

$0 \quad 0 \quad 1 * \cdots 100140$

$\begin{array}{lllllllllllll}0 & 0 & 0 & 0 & 0 & 1 & 0 & 0 & 0\end{array}$

$210^{3}$

$0 \quad 1$

$\begin{array}{llll}1 & 0 & 1 / 1 / 1 & 0\end{array}$

0100

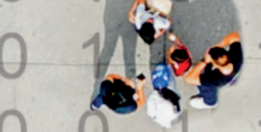

c

01

01

0

01

\section{FINDABLE AND REUSABLE?} DATA DISCOVERY PRACTICES IN RESEARCH

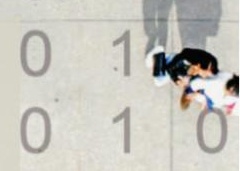

$\begin{array}{lll}0 & 0 & 0\end{array}$

$\begin{array}{lll}0 & 1 & 0\end{array}$

11

0.0

0

0.1

0

00

10

0

0

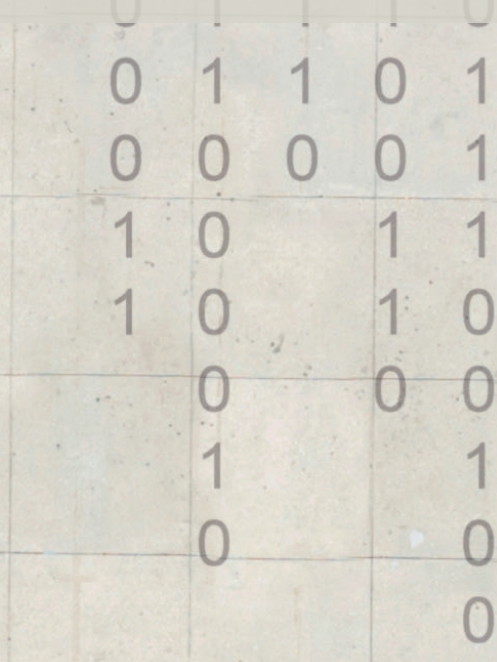

KATHLEEN MARIE GREGORY 



\section{Findable and reusable?}

Data discovery practices in research 
() copyright Kathleen Marie Gregory, Maastricht 2021

Printing: ProefschriftMaken || www.proefschriftmaken.nl

ISBN 978-94-6423-130-4

All rights reserved. No part of this publication may be reproduced, stored in a retrieval system or transmitted, in any form or by any means, electronic, mechanical, photocopying, recording or otherwise, without prior permission of the author or the copyright-owning journals for previous published chapters. 


\title{
Findable and reusable?
}

Data discovery practices in research

\author{
Dissertation \\ to obtain the degree of Doctor at Maastricht University, \\ on the authority of the Rector Magnificus, \\ Prof.dr. Rianne M. Letschert \\ in accordance with the decision of the Board of Deans, \\ to be defended in public on
}

Tuesday, 02 March 2021, at 16:00 hours

by

Kathleen Marie Gregory 


\section{Supervisors}

Prof. dr. S. Wyatt

Prof. dr. P. Groth

Co-Supervisor

Dr. A. Scharnhorst

\section{Assessment Committee}

Prof. dr. J.J.G. Schmeets

Prof. dr. ir. W.E. Bijker

Prof. dr. Christine Borgman

(chair)

Prof. dr. Vivien Petras

University of California, Los Angeles

Humboldt University of Berlin

\section{Funding}

This work was funded by the Dutch Research Council (NWO), grant 652.001.002. 


\section{Table of Contents}

List of Abbreviations $\quad 9$

1. Introduction 10

1.1. Background 13

1.2. Research context, questions and thesis composition 16

1.3. Methodology 20

1.4. Contributions to the field 23

1.5. Terminology and notes $\quad 24$

2. Theoretical framework: User-centered models meet STS 26

2.1. Introduction $\quad 29$

2.2. Information science and STS perspectives in information seeking research 29

2.2.1. Information seeking and information science $\quad 29$

2.2.2. Information seeking and STS 31

2.3. Focus on information science 33

2.3.1. Purpose of information-seeking models in information science 33

2.3.2. Review of selected user-centered models $\quad 34$

2.3.3. Context in user-centered models 38

2.3.4. The information journey 39

2.3.5. Limitations of user-centered models $\quad 41$

2.4. Focus on science \& technology studies $\quad 42$

2.4.1. Sociotechnical networks and practices $\quad 42$

2.4.2. Users 43

2.4.3. Communities $\quad 44$

2.4.4. Context in information research $\quad 46$

$\begin{array}{ll}\text { 2.4.5. Unifying concepts } & 47\end{array}$

2.5. Integrated theoretical framework $\quad 48$

3. Searching data: A review of observational data retrieval practices in selected disciplines $\quad 52$

3.1. Introduction $\quad 55$

3.2. Analytical frameworks $\quad 55$

3.2.1. Framework \#1: A broad view of interactive information retrieval 55

3.2.2. Framework \#2: A broad view of data communities 56

3.3.3. Purpose of the frameworks $\quad 57$

3.3. Methodology 58

3.4. Findings $\quad 58$

3.4.1. Users and needs $\quad 58$

$\begin{array}{ll}\text { 3.4.2. User actions } & 64\end{array}$

3.4.3. Evaluation 69

$\begin{array}{ll}\text { 3.5. Discussion } & 73\end{array}$ 
3.5.1. Users and needs

3.5.2. User actions $\quad 74$

3.5.3. Evaluation $\quad 75$

3.6. Conclusion: Towards a model for data retrieval 76

4. Understanding data search as a socio-technical practice $\quad 78$

4.1. Introduction $\quad 81$

4.2. Bibliometric study and analysis - Method I 82

4.3. Interview design and analysis - Method II 86

4.3.1. Development of interview protocol 86

$\begin{array}{ll}\text { 4.3.2. Interviews } & 86\end{array}$

4.3.3. Interview findings $\quad 88$

4.4. Discussion 102

4.4.1. A broader understanding of the user 102

4.4.2. A broader understanding of data 103

$\begin{array}{ll}\text { 4.4.3. Liminality } & 103\end{array}$

4.4.4. Data search as a sociotechnical practice 104

4.5. Conclusion: Ramifications for system design 104

5. Lost or found? Discovering data needed for research 108

5.1. Introduction 111

5.2. Background 111

5.3. Methodology 113

5.3.1. Survey design $\quad 113$

$\begin{array}{lr}\text { 5.3.2. Sampling and recruitment } & 115\end{array}$

5.3.3. Analysis 116

$\begin{array}{ll}\text { 5.3.4. Reporting } & 117\end{array}$

$\begin{array}{ll}\text { 5.3.5. Limitations } & 117\end{array}$

5.3.6. Ethics and data availability 119

5.4. Results and analysis $\quad 119$

5.4.1. RQ 1: Who are the people seeking data? 119

5.4.2. RQ 2: What data are needed for research and how are those data used? $\quad 125$

5.4.3. RQ 3: How are people discovering data? 132

5.4.4. RQ 4: How are people evaluating and making sense of data for (re)use? $\quad 140$

$\begin{array}{ll}\text { 5.5. Discussion } & 146\end{array}$

5.5.1. Communities of use 146

$\begin{array}{ll}\text { 5.5.2. Interwoven practices } & 147\end{array}$

$\begin{array}{ll}\text { 5.5.3. Social connections } & 148\end{array}$

5.5.4. Practices in flux 149

5.6. Conclusion $\quad 149$

5.6.1. Diversity is normal, not abnormal 149

5.6.2. Communities of use as an entry point to design 150

5.6.3. Metadata to support sensemaking and reuse 151

5.6.4. Tenacity and value of the social 151 
5.7. Supplementary material 153

5.7.1. Appendix A: Survey questionnaire 153

5.7.2. Appendix B: P-value tables 169

5.7.3. Appendix C: Sources used in disciplinary subset 174

6. A dataset describing data discovery and reuse practices in research $\quad 176$

6.1. Background \& summary 179

6.2. Methods $\quad 180$

6.2.1. Questionnaire design 180

6.2.2. Sampling, recruitment and administration $\quad 182$

6.2.3. Ethical review and informed consent 183

$\begin{array}{ll}\text { 6.3. Data records } & 183\end{array}$

6.3.1. Preparation of data files 183

6.3.2. Description of data and documentation files 185

6.3.3. Sample characteristics 186

$\begin{array}{ll}\text { 6.4. Technical validation } & 188\end{array}$

6.4.1. Questionnaire development 188

6.4.2. Questionnaire completion 188

6.4.3. Data quality and completeness 189

6.5. Usage notes 191

6.5.1. Notes for data analysis 191

6.5.2. Potential questions and applications 193

6.6. Code Availability 193

6.7. Reflection: On data papers: Toil, duplication and standardization 197

$\begin{array}{ll}\text { 6.7.1. The what and why of data papers } & 197\end{array}$

$\begin{array}{ll}\text { 6.7.2. Toil } & 198\end{array}$

$\begin{array}{ll}\text { 6.7.3. Affordances and duplication } & 199\end{array}$

$\begin{array}{ll}\text { 6.7.4. Standard formats } & 201\end{array}$

6.7.5. Closing 202

7. Talking datasets: Understanding data sensemaking behaviors 204

7. 1. Introduction 207

$\begin{array}{ll}\text { 7.2. Background } & 208\end{array}$

7.2.1. Sensemaking and information seeking 208

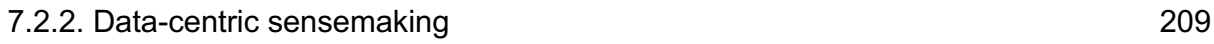

$\begin{array}{ll}\text { 7.2.3. Evaluating data for reuse } & 210\end{array}$

7.2.4. Summarization as a way to understand sensemaking 211

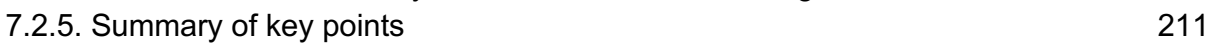

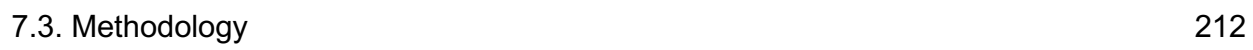

$\begin{array}{ll}\text { 7.3.1. Study design } & 212\end{array}$

$\begin{array}{ll}\text { 7.3.2. Data collection } & 213\end{array}$

7.3.3. Data analysis 216

$\begin{array}{ll}\text { 7.4. Findings } & 218\end{array}$ 
7.4.1. RQ1: What are common patterns in sensemaking activities, both for known and for unknown data, in the initial phases of data-centric sensemaking?

7.4.2. RQ2: How do patterns of data-centric sensemaking afford potential data reuse?

7.5. Discussion 235

7.5.1. Understanding shape 238

7.5.2. "Strange things" as an entry point, not an obstacle 240

$\begin{array}{ll}\text { 7.5.3. Perspectives in placing } & 241\end{array}$

7.6. Study Limitations $\quad 242$

7.7. Conclusions 244

8. Conclusions $\quad 246$

8.1. Introduction 249

8.2. Review of chapters $\quad 249$

8.3. Recurring themes 250

$\begin{array}{ll}\text { 8.3.1 Users and data needs } & 252\end{array}$

8.3.2. Discovering $\quad 254$

8.3.3. Evaluating / Sensemaking 256

8.3.4. Reusing 258

8.3.5. Revisiting STS perspectives $\quad 260$

8.4. Limitations 261

8.5. Future research and open questions 263

8.6. Closing thoughts: Findable and reusable? 264

9. Recommendations and Valorization 266

9.1. Introduction 269

9.2. Recommendations 269

9.2.1 Design recommendations 269

9.2.2. Policy recommendations 274

9.3. Valorization statement 276

References $\quad 281$

Summary / Samenvatting $\quad 325$

Publications list $\quad 335$

$\begin{array}{ll}\text { Acknowledgements } & 339\end{array}$

$\begin{array}{ll}\text { About the author } & 345\end{array}$ 


\section{List of Abbreviations}

\begin{tabular}{|c|c|}
\hline CENS & Center for Embedded Networked Sensing \\
\hline CMOP & Center for Coastal Margin Observation and Prediction \\
\hline CoP & Communities of practice \\
\hline CS & Computer science \\
\hline DANS & $\begin{array}{l}\text { Data Archiving and Networked Services [Dutch research data } \\
\text { repository and center for expertise] }\end{array}$ \\
\hline DBK & $\begin{array}{l}\text { The Data Catalogue, Datenbestandkatalog [German catalog for } \\
\text { social science data] }\end{array}$ \\
\hline ECRs & Early career researchers \\
\hline EDA & Exploratory data analysis \\
\hline FAIR & $\begin{array}{l}\text { Findable, accessible, interoperable and reusable [Guiding } \\
\text { principles for data management] }\end{array}$ \\
\hline $\mathrm{HCl}$ & Human computer interaction \\
\hline IB & Information behavior \\
\hline ICPSR & $\begin{array}{l}\text { Inter-university Consortium for Political and Social Research } \\
\text { [established archive for social science data and research] }\end{array}$ \\
\hline IP & Information practices \\
\hline IR & Information retrieval \\
\hline IS & Information science \\
\hline ISP & $\begin{array}{l}\text { Information Search Process [identifies key stages involved in } \\
\text { information searching] }\end{array}$ \\
\hline MMI & Multiple marginal independence \\
\hline MRCV & $\begin{array}{l}\text { Multiple response categorical variable [variables for survey } \\
\text { questions allowing more than one answer]; R package for statistical } \\
\text { analysis [used to analyze multiple response categorical variables] }\end{array}$ \\
\hline NWO & $\begin{array}{l}\text { Dutch Research Council, Nederlandse Organisatie voor } \\
\text { Wetenschappelijk Onderzoek }\end{array}$ \\
\hline PACS & Picture Archiving and Communication Systems \\
\hline SCOT & $\begin{array}{l}\text { Social Construction of Technology [approach for studying } \\
\text { technology development and adoption] }\end{array}$ \\
\hline SDSS & Sloan Digital Sky Survey \\
\hline SPMI & Simultaneous pairwise marginal independence \\
\hline STS & Science and technology studies \\
\hline
\end{tabular}


CHAPTER 1 
Introduction 
12 | Chapter 1 
Can Malaysia's water supply support its population? Nayla, a water resources researcher, is developing a computational model to find out. To do this, she relies solely on data created by other people, needing satellite imagery, topographical maps, and historical data about population and streamflow. For Nayla, "data collection" is synonymous with finding data created by other people.

How does the researcher in this opening example find the data she needs for her work? Which sources does she consult and which search strategies does she use? How does she make sense of the data she discovers and make decisions about the data's trustworthiness and relevance? In short, what are her practices for discovering data?

Searching for data is not like searching for literature, which has been well-studied and is well-supported (Kern \& Mathiak, 2015). Data discovery requires extra considerations for various actors, such as taking into account issues of data provenance, granularity of content and diverse access restrictions (Chapman et al., 2020). Although there is currently a limited amount of information about how people search for and interact with data (Noy et al., 2019), designing sustainable data search systems is contingent upon understanding and building on extant practices (see Schatzki et al., 2001).

This dissertation expands current knowledge about the practices involved in data discovery by examining what researchers are actually doing as they find, make sense of and use data which they have not created. This work brings together perspectives from information science (IS), computer science (CS) and science and technology studies (STS) to examine these practices with the aim of informing and intervening into the design of search solutions for research data. To do this, it builds on a theoretical framework integrating user-centered models of information seeking behavior with perspectives from STS to paint a rich picture of the diversity of data discovery practices.

\subsection{Background}

Information science and computer science have long studied information seeking behaviors (Sanderson \& Croft, 2012; Courtright, 2007), often employing user-centered models to explore how people search for, evaluate and use information (Case \& Given, 2016). Although not without critique (see Van House, 2004), such models are pervasive in computer and information science. They are used not only to frame research into the search practices of particular groups (i.e. Ellis, et al., 1993; Ellis \& Haugan, 1997) but they are also 
used to practically guide the design and development of new search interfaces (Marchionini \& Komlodi, 1998; Marchionini \& White, 2009).

Despite changes in search technologies and calls for new theoretical framings, the useroriented perspective in information research remains dominant (Savolainen, 2007). Although new metaphors and models have been proposed, for example ecological frameworks (i.e. Nardi \& O'Day, 1999), these perspectives have not yet gained a secure foothold (Tang, et al.; 2019). User-centered models, many developed in the last decades of the 20th century, continue to motivate new research and be relevant for systems design (White, 2016).

User-centered models do not generally mention data explicitly, perhaps recognizing that attempting to differentiate between "data" and "information" places one on a slippery slope. Both information and data are notoriously difficult to define (Floridi, 2010, Borgman, 2015a); and the point at which data become information or vice versa (if at all) is nearly impossible to isolate (Pasquetto et al., 2017).

This difficulty reflects the relational nature of scholarly data. What count as data often lies in the eye of the beholder (Borgman, 2015a), as data are defined differently within particular epistemic communities (Borgman, Wallis, Mayernik, et al., 2007; Wallis et al., 2007) and at different timepoints in the inquiry process (Leonelli, 2015). A computational biblical scholar, for example, will have very different notions of data than a lepidopterist studying the evolution of butterflies. Leonelli proposes that scientific data are best understood as a relational category defined by both their perceived usefulness to a researcher in making evidential claims and by their portability, or ability to circulate to establish those claims (Leonelli, 2015).

Across disciplines, work is being done to make data "portable" in new ways. Research data are made available in multidisciplinary or discipline-specific data repositories (Tenopir et al., $2011 ; 2015)$, often at varying levels of granularity. New ontologies and metadata schemas are being created (Sansone et al., 2017, DataCite Metadata Working Group, 2019) or modified (Gray et al., 2017; Benjelloun et al., 2020) to describe data. Access to data is also mediated differently, as researchers weigh which data to make open when and for whom (Levin \& Leonelli, 2017) and consider how to make sensitive data available while protecting the privacy of research participants (see European Commission, 2018b). These practices of how data are made available, how they are described, and how they can be accessed have potential implications for data search (Koesten, Mayr, et al., 2019). 
The very idea of data search or data seeking is intertwined with claims about the potential of data reuse, using data created by others. These claims are multiple and have been made by funders, researchers, policy makers and publishers. Some see data reuse as an antidote to problems with reproducibility in science (see Pasquetto et al., 2017; Baker, 2016), providing an avenue for the validation of experimental findings. Others tout the economic benefits of data reuse, claiming that millions of euros and research hours could be saved by limiting the amount of possibly redundant, and almost always costly, data collection (European Commission, 2018a). Still others embrace the possibilities that shared data pools offer for new data-driven approaches to tackle the most complex problems of our time (CODATA, n.d., United Nations, n.d.).

There are many suppositions and assumptions underlying these claims. It is assumed that researchers will share their data to build up the needed data supplies (Borgman, 2012). It is assumed that data can travel to new situations and be understandable to actors, both humans and machines, who were not involved in their creation (Leonelli, 2016; Garnett, 2017). Another assumption predicating reuse is that data will actually be discovered by researchers, although relatively little empirical work exists to support this belief.

Contrary to these presumptions, data do not flow smoothly and unproblematically to new situations (Leonelli, 2016). As I suggest through the course of this dissertation, data discovery and reuse rely on dynamic relationships between people, technology, materials and policy. Most research into data discovery to date focuses on one aspect of this relationship: the technological challenges involved in making data searchable. Motivated by the fact that techniques for document-based retrieval do not work well for structured data, computer scientists have experimented with applying keyword searches across tables rather than metadata (Zhang \& Balog, 2018), developing ontologies and standards (OhnoMachado et al., 2017; Sansone et al., 2017) and creating general (Noy et al., 2019; Scerri, et al., 2017) and discipline-specific (Park, et al., 2017; Megler \& Maier, 2013) data search tools.

Despite the wealth of research investigating information seeking practices (Julien \& Duggan, 2000), data discovery practices, how people are discovering, making sense of and reusing data, are just beginning to be empirically investigated. A small number of studies examines how individuals seek and evaluate data within disciplines (Zimmerman, 2003; Davis et al., 2014; Martin-Mora, 2020), across data-related professions (Koesten et al., 2017), or within data repositories (Borgman et al., 2020; Murillo, 2015; Wu et al., 2019). Details about data discovery practices are often embedded within investigations of other data practices, such 
as explorations of data sharing, data management, and data reuse in specific disciplines (Wynholds, et al., 2012; Wallis et al., 2013; Curty, et al., 2016).

This dissertation addresses this gap by directly examining how researchers, across disciplines, discover, understand and use data which they do not create themselves. Usercentered models of information seeking provide an overarching framework for this work, while STS is used to engage with these models to explore the sociotechnical nature of data discovery.

\subsection{Research context, questions and thesis composition}

\subsubsection{Research context}

This research was part of an interdisciplinary project, Re-SEARCH, funded by the Dutch Research Council (NWO, Grant number 652.001.002). The purpose of Re-SEARCH was to investigate and develop search solutions for research data. Researchers from three universities, a data archive and an academic publisher pursued separate lines of research within the project. My work on data discovery and reuse practices took place alongside research into the development of semantic technologies and relevance ranking algorithms for data search.

The academic publisher, Elsevier, provided logistical support for all projects, with the aim of implementing findings from all research lines into their prototype search engine for research data, DataSearch ${ }^{1}$. One of the goals of my research was to contribute to this project by informing the design of this search engine.

\subsubsection{Research questions}

The principal questions addressed in this dissertation can therefore be summarized as:

(1) How do researchers across disciplines discover, make sense of and (re)use data in their work?

(2) How can insights from science and technology studies be used to deepen usercentered perspectives for understanding data discovery practices?

\footnotetext{
${ }^{1}$ DataSearch has now been integrated into another Elsevier platform, Mendeley Research Data, and is available at: https://data.mendeley.com/research-data/
} 
(3) How can these findings about data discovery practices inform the design of systems for data search?

\subsubsection{Thesis composition}

The dissertation builds on four empirical studies, resulting in five published articles, in which I investigate these questions (Table 1.1). In this thesis, I consolidate the work presented in these studies and examine it in relation to the theoretical framework presented in Chapter 2. The contributions of other researchers in these publications are detailed in Table 1.2.

\begin{tabular}{|c|c|}
\hline & Citation \\
\hline Chapter 3 & $\begin{array}{l}\text { Gregory, K., Groth, P., Cousijn, H., Scharnhorst, A., Wyatt, S. (2019). Searching data: A } \\
\text { review of observational data retrieval practices in selected disciplines. Journal of the } \\
\text { Association of Information Science and Technology, 70, 419-432. } \\
\text { https://doi.org/10.1002/asi.24165 }\end{array}$ \\
\hline Chapter 4 & $\begin{array}{l}\text { Gregory, K., Cousijn, H., Groth, P., Scharnhorst, A., Wyatt, S. (2019). Understanding } \\
\text { data search as a socio-technical practice. Journal of Information Science. } \\
\text { https://doi.org/10.1177/0165551519837182 }\end{array}$ \\
\hline Chapter 5 & $\begin{array}{l}\text { Gregory, K., Groth, P. Scharnhorst, A., Wyatt, S. (2020). Lost or found? Discovering } \\
\text { data needed for research. Harvard Data Science Review. } \\
\text { https://doi.org/10.1162/99608f92.e38165eb }\end{array}$ \\
\hline Chapter 6 & $\begin{array}{l}\text { Gregory, K. (2020). A dataset describing data discovery and reuse practices in } \\
\text { research. Scientific Data, } 7(232) \text {. https://doi.org/10.1038/s41597-020-0569-5 }\end{array}$ \\
\hline Chapter 7 & $\begin{array}{l}\text { Koesten, L. }{ }^{*} \text {, Gregory, K. }{ }^{*} \text {, Groth, P., Simperl, E. (2020). Talking datasets - } \\
\text { Understanding data sensemaking behaviours. International Journal of Human-Computer } \\
\text { Studies. https://doi.org/10.1016/j.jjhcs.2020.102562. *Equal contributions }\end{array}$ \\
\hline
\end{tabular}

Table 1.1. Citations for the five published articles included in this dissertation. 


\begin{tabular}{|c|c|c|c|c|c|}
\hline & Chapter 3 & Chapter 4 & Chapter 5 & Chapter 6 & Chapter 7 \\
\hline $\begin{array}{l}\text { Theory } \\
\text { development }\end{array}$ & $\begin{array}{l}\text { Helena Cousijn } \\
\text { Paul Groth } \\
\text { Andrea Scharnhorst } \\
\text { Sally Wyatt }\end{array}$ & $\begin{array}{l}\text { Helena Cousijn } \\
\text { Paul Groth } \\
\text { Andrea Scharnhorst } \\
\text { Sally Wyatt }\end{array}$ & & & Laura Koesten \\
\hline $\begin{array}{l}\text { Research } \\
\text { design }\end{array}$ & & $\begin{array}{l}\text { Helena Cousijn } \\
\text { Paul Groth } \\
\text { Andrea Scharnhorst } \\
\text { Sally Wyatt }\end{array}$ & $\begin{array}{l}\text { Paul Groth } \\
\text { Ricardo Moriera } \\
\text { Andrea Scharnhorst } \\
\text { Sally Wyatt }\end{array}$ & & $\begin{array}{l}\text { Paul Groth } \\
\text { Laura Koesten }\end{array}$ \\
\hline Data analysis & & & & & Laura Koesten \\
\hline Writing & & & & & Laura Koesten \\
\hline $\begin{array}{l}\text { Review and } \\
\text { editing }\end{array}$ & $\begin{array}{l}\text { Helena Cousijn } \\
\text { Paul Groth } \\
\text { Andrea Scharnhorst } \\
\text { Sally Wyatt }\end{array}$ & $\begin{array}{l}\text { Helena Cousijn } \\
\text { Paul Groth } \\
\text { Andrea Scharnhorst } \\
\text { Sally Wyatt }\end{array}$ & $\begin{array}{l}\text { Paul Groth } \\
\text { Andrea Scharnhorst } \\
\text { Sally Wyatt }\end{array}$ & $\begin{array}{l}\text { Paul Groth } \\
\text { Andrea Scharnhorst } \\
\text { Sally Wyatt }\end{array}$ & $\begin{array}{l}\text { Paul Groth } \\
\text { Elena Simperl }\end{array}$ \\
\hline
\end{tabular}

Table 1.2 Overview of contributions of co-authors and others to the published articles

Chapters 3 through 7 consist of reprints of the published articles. Table 1.3 presents an overview of the purposes, relevant research questions, methodologies and ethical approval details (when appropriate) for Chapters 2 through 7. Ethical approval was received from university ethics boards for all studies involving human subjects. Chapter 8 summarizes and discusses key findings and conclusions drawn from the entirety of the research presented in the dissertation. Chapter 9, the final chapter, synthesizes the design recommendations proposed throughout the thesis, poses potential policy considerations and reflects on the broader impact of this research. (Other publications produced during the course of completing this doctoral work are also discussed in Chapter 9 and are listed at the end of the dissertation). Each chapter is now briefly summarized, before the methodological approaches are described in more detail. 


\begin{tabular}{|c|c|c|c|c|}
\hline & Purpose & Research questions & Methodologies & Ethical approval \\
\hline Chapter 2 & theoretical framework & RQ2: theory & & \\
\hline Chapter 3 & empirical research & RQ1: practices & $\begin{array}{l}\text { analytical literature } \\
\text { review }\end{array}$ & \\
\hline Chapter 4 & empirical research & $\begin{array}{l}\text { RQ1: practices } \\
\text { RQ3: recommendations }\end{array}$ & $\begin{array}{l}\text { bibliometric analysis; } \\
\text { interviews }\end{array}$ & Maastricht University \\
\hline Chapter 5 & empirical research & $\begin{array}{l}\text { RQ1: practices } \\
\text { RQ3: } \\
\text { recommendations }\end{array}$ & survey & Maastricht University \\
\hline Chapter 6 & $\begin{array}{l}\text { data descriptor; } \\
\text { reflection }\end{array}$ & & & \\
\hline Chapter 7 & empirical research & $\begin{array}{l}\text { RQ1: practices - } \\
\text { sensemaking } \\
\text { RQ3: } \\
\text { recommendations }\end{array}$ & $\begin{array}{l}\text { interviews; } \\
\text { think-aloud task; } \\
\text { observations }\end{array}$ & University of Southampton \\
\hline RQ summary: & $\begin{array}{l}\text { RQ1: Practices - how } \\
\text { RQ2: Theory - how car } \\
\text { RQ3: Recommendatio }\end{array}$ & $\begin{array}{l}\text { researchers across disci } \\
\text { insights from STS be use } \\
\text { - how can these findings }\end{array}$ & $\begin{array}{l}\text { plines discover, make } \\
d \text { to nuance user-cente } \\
\text { inform the design of } d\end{array}$ & $\begin{array}{l}\text { se of and (re)use data? } \\
\text { perspectives? } \\
\text { search systems? }\end{array}$ \\
\hline
\end{tabular}

Table 1.3 Overview of chapter purposes, methodologies and relevant research questions

Chapter 2 introduces my theoretical framework, which integrates user-centered models of information seeking common in information science with perspectives from STS. The chapter addresses how information seeking has been studied in both of these disciplines, before reviewing influential user-centered models and discussing how STS has approached key elements implicated in them, namely users, communities and context. I then discuss how I integrate these perspectives to frame my research.

Chapter 3 presents the first piece of empirical work, an analytical review of the literature documenting how individuals discover and evaluate observational data in selected disciplines. This chapter employs an iteration of the theoretical framework presented in Chapter 2, to suggest that sociotechnical practices of data discovery cannot be adequately examined through user-centered models alone.

Chapter 4 further probes the sociotechnical nature of data discovery, drawing on a bibliometric analysis and semi-structured interviews conducted with data seekers across disciplines. This chapter bridges areas that are often disconnected, providing an example of how scientometric studies can inform and shape qualitative research.

Building on the findings of the first two studies, the fifth chapter presents the results of a global survey investigating the data discovery practices of individuals involved in research. The work presented in this chapter incorporates descriptive statistics and pairwise 


\section{0 | Chapter 1}

correlations to identify commonalities and differences in practices; it also draws heavily on qualitative, open-response answers to represent the complexity and diversity of discovering data.

The sixth chapter represents my first-person experience with sharing and preparing data for reuse. This chapter consists of two parts. The main body of the chapter, which was published as a data descriptor paper, details the openly-available data collected from the survey described in Chapter 5 (Gregory, 2020a). This is followed by a reflection piece, in which I consider the process of sharing and describing my data, the effect of these practices on data discovery and reuse and the role of data papers in the broader landscape of scholarly communication.

Chapter 7 presents the final piece of empirical work, a collaboration with experts in humancomputer interaction at the University of Southampton ${ }^{2}$ and the University of Amsterdam, which investigates the initial stages of data-centric sensemaking. In this work, we analyze how researchers make sense of both known and unknown data and identify common activity patterns to propose a series of recommendations to support sensemaking.

The dissertation ends with two concluding chapters. In Chapter 8, I examine key findings and conclusions drawn from all studies in light of the theoretical framework introduced in Chapter 2, before I suggest lines for future research. Chapter 9 synthesizes the recommendations made throughout the dissertation, proposes potential considerations for policy work and reflects on the broader impact of this research.

\subsection{Methodology}

Many valuable findings in STS come from in-depth ethnographic studies providing "thick descriptions" (Geertz, 1973) of particular cases. This approach is extremely rich and has yielded insights which have informed policy and technological developments (Wyatt \& Balmer, 2007). Despite this, given my research aims and the interdisciplinarity of the research topic, I took a different methodological approach.

My work seeks to both represent the complexity of practice while at the same time finding patterns which could be used to inform the design of data search systems, or, as Hine summarizes, to perform "analysis adequate to guide intervention beyond a particular case"

\footnotetext{
${ }^{2}$ As of February 2020, the researchers from the University of Southampton are employed at King's College London.
} 
(Hine, 2007, p.652). This involves balancing between identifying commonalities potentially useful for systems design while also providing rich descriptions reflective of actual practice.

To address this challenge, I employ a mixture of quantitative and qualitative approaches, often within the same study, to identify common patterns while also highlighting differences. In an effort to further communicate my findings to a variety of audiences interested in data discovery (i.e. repository managers, librarians, computer scientists, research data managers), I present my qualitative data in ways normally reserved for quantitative data, such as tables and visualizations.

The methods used throughout the dissertation built sequentially upon each other, as shown in Figure 1.1. A systematic literature search was conducted to gather the literature analyzed in Chapter 3. This literature corpus was further analyzed using bibliometric techniques and informed the development of a protocol for semi-structured interviews with data seekers (Chapter 4). The findings from both the literature analysis and the interviews informed the design of the survey questionnaire. Data from the survey are analyzed and discussed in Chapter 5; Chapter 6 further details the survey data, as well as the process used to prepare the data for sharing and archiving. 
22 | Chapter 1

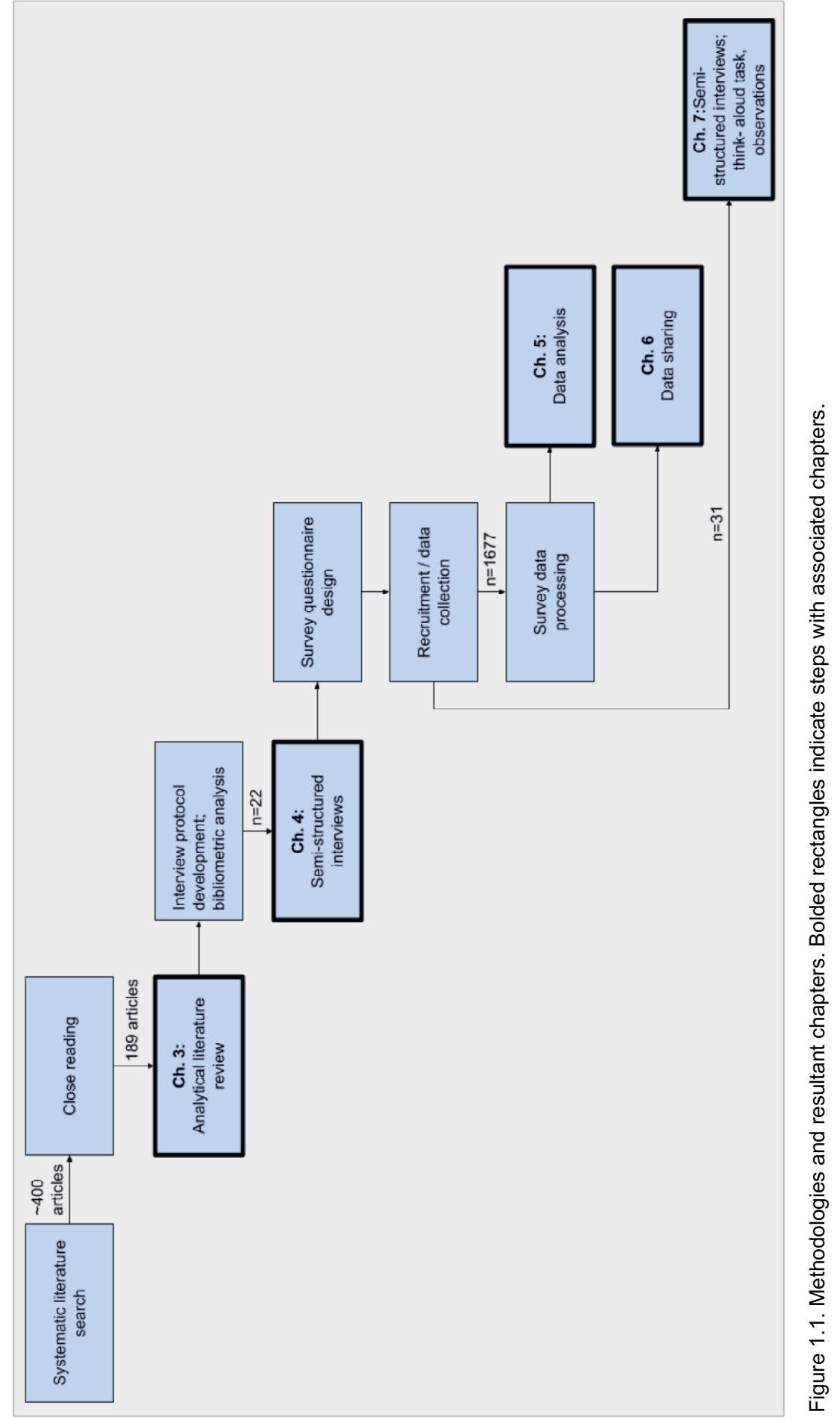


Identifying researchers who engage in data reuse and data discovery is challenging (Park et al., 2018; Yoon, 2014a). The collaboration with Elsevier facilitated the recruitment of research participants for both the interviews and for the survey study. Participants for the interview study described in Chapter 4 were drawn from a pool of individuals who had visited the website of Elsevier's prototype search engine, DataSearch, and had indicated they would be willing to provide feedback. Survey respondents were recruited from the list of authors publishing an article indexed in Elsevier's Scopus literature database within the last three years.

Of the 1,677 respondents to the survey, 1,057 indicated that they would be willing to participate in future research on the topic. In my collaborative work with researchers from the University of Southampton investigating data-centric sensemaking (Chapter 7), we recruited interview participants from this pool of survey respondents. In this study, we asked researchers to describe and interact with both familiar and unfamiliar data. Here, we also combined quantitative and qualitative approaches, complementing our semi-structured interviews and observations with a think-aloud task and a quantitative analysis of participants' interactions with data.

\subsection{Contributions to the field}

First and foremost my work provides evidence of practices of data discovery, augmenting the technical discourse of data search by examining researchers' practices. This dissertation empirically investigates the data which researchers use, the purposes for which those data are sought, and the sources and search strategies employed to locate those data. It also explores how researchers make sense of data and nuances the factors influencing decisions about data reuse. This dissertation highlights the interplay between the social and technical spheres in these practices and their relations with other research and data practices. It also describes in detail an openly available dataset which can be used as the basis for future research.

Secondly, this work can inform the design of community-centric discovery tools and policies for data reuse. Design recommendations for data repositories and discovery systems are given throughout the dissertation and are summarized in the concluding chapter, as are recommendations and considerations for policy makers.

Third, this research provides a connecting point between the various disciplinary domains and stakeholders involved in researching data discovery. This is achieved through 


\section{4 | Chapter 1}

interweaving quantitative and qualitative methods, presenting findings in a variety of ways and introducing a framework integrating information science and STS perspectives. The chapters in this dissertation were originally published in journals catering to various disciplinary subfields: information science, data science and human-computer interaction, further facilitating communication across disciplines.

Fourth, the integrated theoretical framework which I apply here could potentially be adopted as a methodology to complement purely user-centered approaches in other areas of information research. This framework consolidates broad perspectives from user-centered models and core STS findings to pose questions that can be used to explore both data and information practices.

Ultimately, this dissertation contributes to the broader discourse on data discovery, data sharing and data reuse and the position of these practices in scholarly communication and knowledge production. Through both the evidence and analyses presented in these chapters, I emphasize that diversity is normal, not abnormal when it comes to discovering and reusing data, recognizing that a one-size-fits-all approach to developing tools, documentation guidelines and policies does not resonate with actual practice.

\subsection{Terminology and notes}

I gradually came to embrace the term "data discovery," which I use in this introductory chapter, to refer to the entire process of finding, evaluating and using data in research. In other chapters, I use the language of "data retrieval," "data search," or "data seeking" to refer to this same process, although each of these terms arguably has a more specific meaning. Applying distinctions drawn from the information-seeking literature (Wilson, 1999), "data search" or "data retrieval" could refer to the process of individuals actively engaging with search systems to locate data. "Data seeking" would refer to a broader set of goaldirected practices and resources, which do not only involve interacting with systems. "Data discovery" is a term that allows for serendipity to occur, not limiting practices to goaldirected behaviors. I adopted this term, as it is more inclusive of the diverse, at times serendipitous practices which I observed throughout my research.

Similarly, although I use the broad term models of information-seeking behavior here and in the following chapter, some of these models were developed within the sub-field of interactive information retrieval, as discussed further in Chapter 2. In my initial research, particularly in Chapter $3, \mathrm{I}$ refer to them using this more specific terminology. 
The following chapter presents the theoretical approach which frames the majority of the work presented in this dissertation. Although it is presented as a cohesive whole in the Chapter 2, this framework was iteratively refined and formalized throughout my research.

The language and references used in the empirical chapters reflect this development. Early chapters do not specifically refer to the model of the information journey (Blandford and Attfield, 2010), which structures the framework in Chapter 2; rather, I reference specific models of information seeking and retrieval. Chapter 3 in particular utilizes an earlier iteration of the framework.

Finally, early empirical chapters call for the development of new models for describing data discovery practices. While this was an initial possible outcome of my research, this goal changed, as I instead saw space for frameworks for investigating the sociotechnical nature of data discovery practices. Chapter 2 represents one possibility for such a framework.

I now turn to describing my theoretical approach and its foundations in more detail, building first on explorations of information seeking in both information science and science and technology studies. 


\section{CHAPTER 2}


Theoretical framework:

User-centered models meet STS 
28 | Chapter 2 


\subsection{Introduction}

Researchers from across disciplinary domains have explored how individuals encounter, understand and engage with information using multiple theoretical approaches (Blandford \& Attfield, 2010). Work in information science (IS) and science and technology studies (STS), for example, has explored information discovery through the lens of cognitive, user-centered perspectives or theories influenced by social constructivism. Studies have used these approaches to investigate information activities in everyday life (i.e. Savolainen, 1995), in workplaces and organizations (i.e. Choo, 2007), and in academic and research settings (i.e. Ellis \& Haugan, 1997; Palmer, 1999).

This dissertation examines data discovery practices in research with the intent to inform the design of data search tools. I situate this work within the well-investigated area of information-seeking, bringing together perspectives from both information science and STS. This allows me to build on existing work in information-seeking across disciplines as well as to facilitate the communication of my findings to a range of stakeholders, including systems designers, who often incorporate principles of user-centered design in their work (Holtzblatt \& Beyer, 2017). At the same time, it allows me to experiment with how STS can help to counter idealized notions of user-centered data search.

This chapter presents this theoretical framework and its foundations in more detail. The chapter opens with an overview of information-seeking research in both information science and science and technology studies. Each perspective, IS and STS, is then explored in more depth, beginning with a review of five influential user-centered models. I then discuss how STS has approached key concepts implicated in these models, namely users, communities and context. The chapter ends by bringing together two cornerstones drawn from these approaches to structure the framework - a modified version of the information journey model (Blandford \& Attfield, 2010) and a series of guiding concepts and questions drawn from STS.

\subsection{Information science and STS perspectives in information seeking research}

\subsubsection{Information seeking and information science}

Information science is a broadly defined field with close ties to a diversity of other disciplines, including computer science, library science and telecommunications (Yan, 2011). The field is 
primarily concerned with the representation, classification and storage of information, as well as how information is sought, retrieved and used (Stock \& Stock, 2013).

Information seeking has been a topic of significant interest within information science (Courtright, 2007; Wang, 2011), particularly within the areas of information behavior (IB) and information retrieval (IR) research. These two fields are closely-related, yet separate, with different areas of emphasis (Stock \& Stock, 2013).

Information behavior can be defined as the broad area of research examining information needs, seeking and use (Courtright, 2007). Wilson proposed a nested model to further classify research in this area into information-seeking behaviors and information search behaviors (Wilson, 1999, Figure 2.1). Information-seeking is generally taken to refer to activities undertaken to acquire information to fulfill a need, want or gap in knowledge (Case \& Given, 2016). Research into information search behaviors, a subset of informationseeking, focuses on specific interactions between users and search technologies (Wilson, 1999). Traditionally, researchers examining information seeking behaviors from the IB perspective tend to be trained in the field of library and information science (Jansen \& Rieh, 2010).

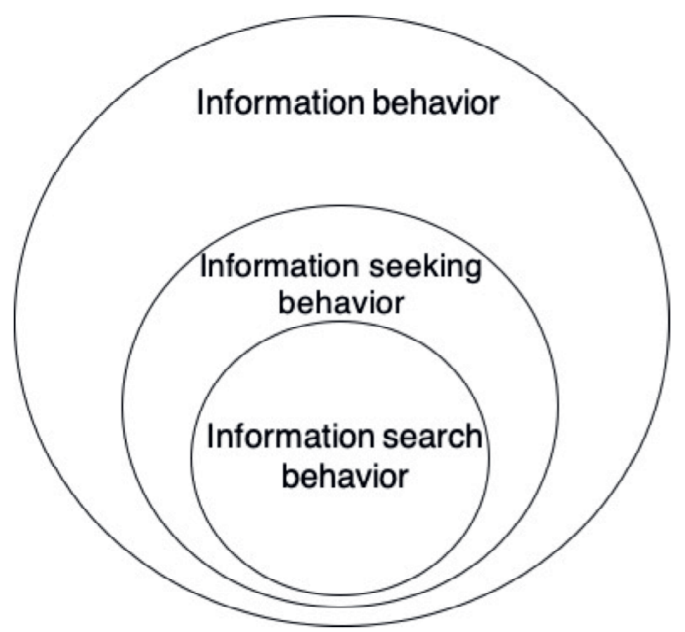

Figure 2.1. Nested model classifying the field of information behavior research. Adapted from Wilson, 1999.

Information retrieval is defined as the study of finding and extracting information from large collections of computerized resources to meet an information need (Jansen \& Rieh, 2010). 
Researchers engaging in information retrieval research are usually trained in computer science or related programs and focus on developing new technologies and search systems (White, 2016). IR is therefore inextricably linked with computer science (Sanderson \& Croft, 2012), although it is considered by some to be a well-crystallized subfield of information science, with its own conferences and journals (Stock \& Stock, 2013; Jansen \& Rieh, 2010). The research area of interactive information retrieval draws on work from both IB in information seeking and search behaviors as well as work in IR regarding the development of methods to support interacting with electronic resources (Ruthven, 2008).

Jansen \& Rieh analyze the similarities, differences and overlaps between the theoretical constructs used in both IB and IR research along three lines: their treatment of people, information and technology (2010). One difference between IB and IR research lies in the representation of information. IR research tends to adopt an information-as-thing perspective, where information is viewed as a physical object (i.e. a document) which can be acted upon, for example by indexing, parsing or encoding (Buckland, 1991). IB research, however, views information as something that can be multiply defined, often only in relation to its context. Both IB and IR research converge on their conceptualization of people, who are defined as "users" of information systems (Jansen \& Rieh, 2010).

This convergence reflects the predominant user-centered perspective within information science. The user-centered view has held sway since the mid-1980's (Tang et al.; 2019; Savolainen, 2007), when the field was seen to have moved from a systems-oriented view to one foregrounding the information seeker's standpoint (Dervin \& Nilan, 1986). The predominance of the user-centered perspective is also reflected in the focus on usercentered design within neighboring fields in computer science (Norman \& Draper, 1986; Holtzblatt \& Beyer, 2017).

\subsubsection{Information seeking and STS}

STS is a diverse and interdisciplinary field whose boundaries are difficult to define. Growing out of domains such as sociology, anthropology, and the philosophy and history of science, STS has the broad aim of studying the processes and outcomes of science and technology (Sismondo, 2004). Researchers in STS employ a range of diverse and innovative methodologies (Wyatt et al., 2007), although ethnographic case studies tend to form the core of STS work (Sismondo, 2004). 
Perspectives from STS have been applied to understand practices in various domains, including healthcare (i.e. see Webster \& Wyatt, 2020), energy and sustainable development (i.e. Shove, et al., 2015) and information science (i.e. Ribes et al., 2019, Bowker \& Star, 1999). The focus of STS-inspired research within information science is not typically on the user or on the system. Rather, these studies reflect the STS tenet that society and technology are not separate entities, but are instead co-constituents of a seamless web of dynamic social, material, political and economic elements (see van House, 2004). Scientific knowledge itself is not a naturally existing phenomena in this view, but is rather something that is socially and actively constructed (Sismondo, 2004).

These views are apparent within the sub-field of "information practices" (IP) research, an approach that began to gain increased attention within information science during the first decade of the 21st century (Savolainen, 2007). This approach examines how informationseeking activities are influenced and shaped by both social and cultural factors (Tuominen et al., 2005). Information seeking and use are seen as constructing activities, as is information itself, which Dervin defines as the "sense that is made" at particular moments in space and time (1983).

The information practices approach emphasizes that individuals act as members of communities and social groups, often in diverse roles, rather than as isolated actors, and that information seeking is a social practice (Talja \& Hansen, 2006). The focus in information practices research is not on the users per se, but rather on the sociotechnical infrastructures, practices and contextual factors surrounding and shaping information seeking (Savolainen, 2007). The boundaries between the IP and user-centered approaches, particularly within interactive information retrieval, are not always as clear cut as this presentation may suggest. Despite possible overlaps, communication between these approaches remains a recognized challenge (Tabak, 2014; Blandford \& Attfield, 2010).

I now examine both perspectives in more detail, beginning with a review of influential models of user-centered information-seeking, situated within the area of IB/IR. I then explore how STS has studied key elements implicated in user-centered models, namely users, communities and context. 


\subsection{Focus on information science}

\subsubsection{Purpose of information-seeking models in information science}

A multitude of conceptual models have been developed over the last sixty years to describe information-seeking behaviors. Such models usually consist of diagrams describing relationships among concepts (Case \& Given, 2016), and are rooted in the IB paradigm, although they have also been developed and used in (interactive) information retrieval and human computer interaction (see Xie, 2008; White, 2016).

Models have varying levels of specification; some provide a high-level summary of the elements and activities involved in finding and using information (Xie, 2008). Other models are more stage-like and analytical in their design (Savolainen, 2018), representing processes and strategies users take when searching for information (White, 2016). The majority of models focus on search behaviors (Järvelin \& Wilson, 2003) and posit a user taking action to fulfill an information need.

Different types of models serve different research purposes. Some models facilitate developing research questions, interpreting observations, or systematizing knowledge (Järvelin \& Wilson, 2003). Others are more suitable for developing and testing hypotheses (Xie, 2008) or have been used to inform the design of search interfaces (see Marchionini \& Komlodi, 1998) or to investigate the search practices of particular groups (i.e. Ellis, et al., 1993; Ellis \& Haugan, 1997).

Both broad and more stage-like models have provided unique contributions, leading to understanding information seeking as an iterative, dynamic process defined through interaction (i.e. Saracevic, 1996; 1997; Ingwersen, 1992; 1996; Bates, 1989). In order to contextualize these models and their contributions, I now review five influential models of information-seeking, many developed during the last decade of the 20th century. These models were chosen as they are some of the most highly-cited models (Xie, 2008), they span both the IB and interactive IR traditions, and they bring forth ideas (indicated in bold) which informed the research presented in subsequent chapters of this dissertation.

After reviewing these models, I present the model of the information journey (Adams and Blandford, 2005; Blandford \& Attfield, 2010), which brings together many of the insights from the reviewed models and provides the structure for the integrated framework presented in Section 2.5 . 


\subsubsection{Review of selected user-centered models}

Three of the most pivotal models providing a high-level view of information seeking include Ingwersen's cognitive model of information transfer $(1992,1996)$, Saracevic's stratified model $(1996,1997)$ and Belkin's episodic model $(1993,1996)$.

In his model, Ingwersen emphasizes the "cognitive transformations" and communication of "cognitive structures" that occur between five elements involved in information seeking: a user's cognitive space, the user's socio-organizational environment, the interface/intermediary, information objects, and the search system $(1992,1996)$.

Cognitive structures, also referred to by Ingwersen as knowledge structures, are the systems of categories and concepts that constitute a human's (or a machine's) model of the world.

These structures are not static, but are determined at a particular point in time by an individual's social/collective experience (Ingwersen, 1992). In information seeking, transformations in cognitive structures are performed by various human actors involved with the different elements. These include users, but also other actors, i.e. systems designers and indexers.

Ingwersen's model brings together systems-oriented and user-oriented perspectives in information science, as it includes information objects (i.e. text/knowledge representations, pictures, semantic entities) as well as elements related to the user, such as task, cognitive state and information need. It also names contextual elements in the socio-organizational environment, such as scientific or professional domains, which influence a user's interactions.

Saracevic's stratified model of information retrieval interaction (1996) also incorporates elements representing systems and users. This model depicts interaction in information seeking as an interplay between levels representing users and systems that occurs at a surface level. Different levels, or strata, describe interactions on the user side (i.e. situational, affective and cognitive interactions) and the system side (i.e. interactions involved in content, processing and engineering) (Figure 2.2). 


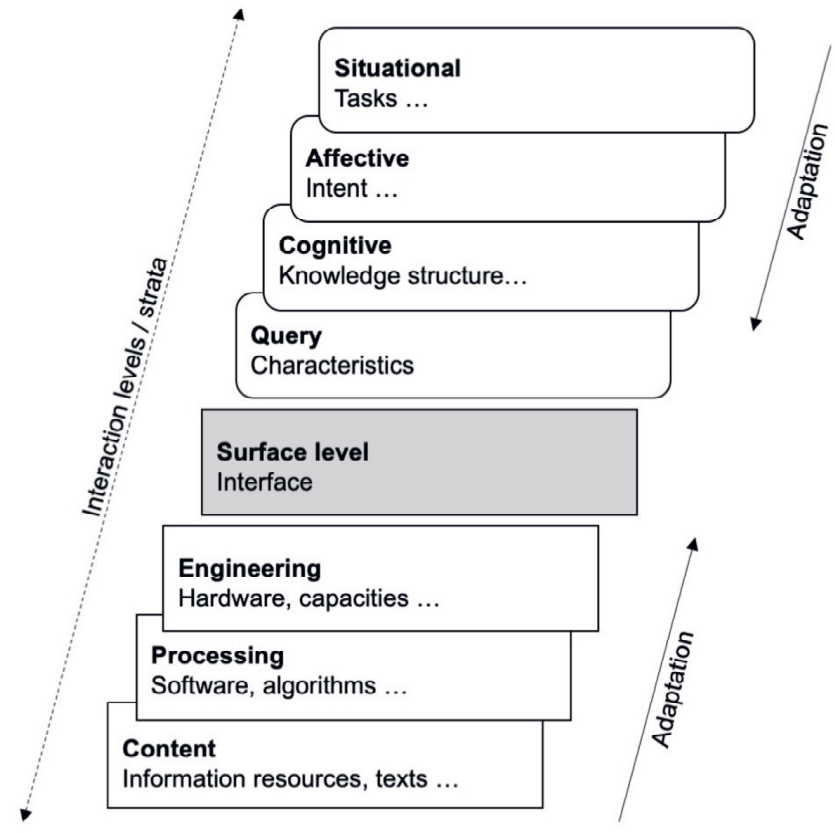

Figure 2.2. Stratified model of information retrieval interaction. Adapted from Saracevic, 1996.

A key part of this model is that it portrays search as a dynamic conversation between a user and a search system that occurs at the surface interface. Both the user and the system contribute to this conversation, the user by entering search queries, browsing, navigating etc. and the system by providing results or suggestions.

Another unique contribution is that of "adaptation," the recognition that changes in one level of interaction will affect the other levels. Poor indexing in the content level, for example, may result in poor results, even with a good algorithm (White, 2016).

Belkin's episode model of interaction with texts (1996, Figure 2.3) draws on his earlier work, which characterizes information-seeking along four dimensions with associated information seeking strategies (1993). 


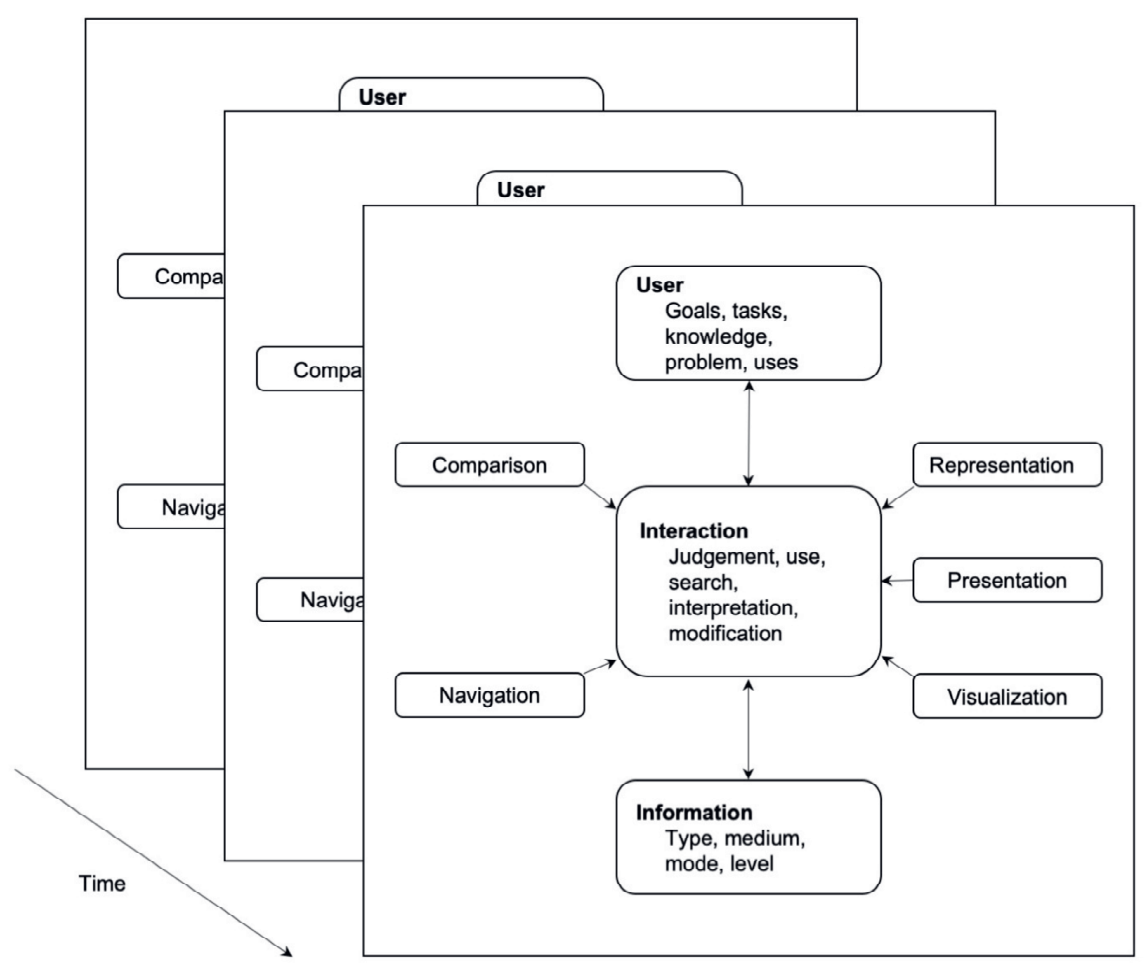

Figure 2.3. Episode model of interaction with texts. Adapted from Belkin, 1996.

This model emphasizes the interactions involved in information-seeking and recognizes that a searcher will employ a wide variety of search strategies over multiple episodes. It is unique in that it includes the user as an inherent part of the system (Xie, 2008), which is composed of three components: the searcher, the knowledge resource and an intermediary (human or machine) that supports the user's interactions. Unlike the models reviewed so far, the only elements depicted in this model are the user, the information and the interactions, which are supported by various processes: representation, comparison, presentation, navigation and visualization.

Shifting focus, I look now at two of the most well-known models detailing the processes or activities involved in searching for information: Bates's berrypicking model (1989) and Kuhlthau's model of the information search process (1991).

Bates' berrypicking model (1989) provides a different interpretation of the information seeking process, as she challenges the traditional document matching model of information retrieval. Her model depicts a distributed information space; a searcher moves through this 
space, collecting bits of information as if picking berries from bushes. A searcher's path evolves in response to cues picked up along the way in text and through context (Figure 2.4). The searcher continues gathering information until the information need becomes redundant - either the searcher is satisfied or her need has changed and is no longer important.

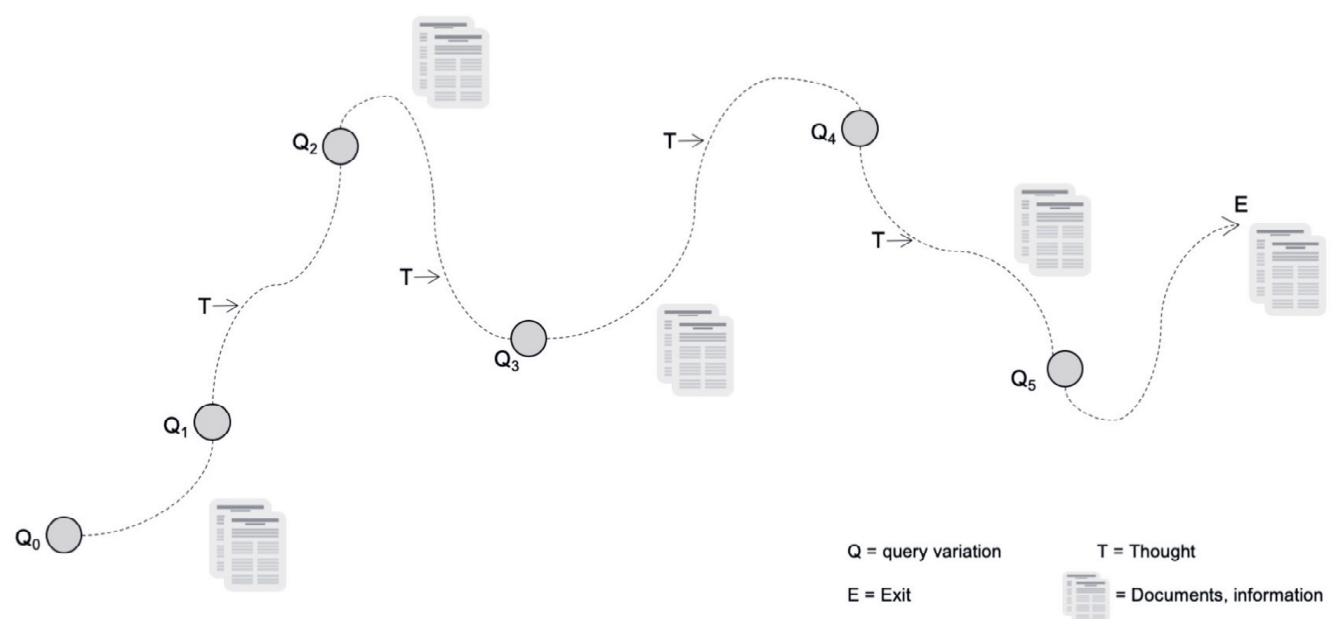

Figure 2.4. An evolving, berrypicking search. Adapted from Bates, 1989 and the depiction of this model in White, 2016.

The key contributions of the berrypicking model are twofold. First, it highlights the dynamic nature of information needs, recognizing that they are formed by cues collected during an evolving search process. Second, it suggests that information is distributed and a searcher must piece together relevant bits, rather than finding a single best set of results.

Kuhlthau's model of the information search process (ISP) was based on a series of longitudinal studies of students and library patrons (1991). She identifies six key stages involved in the search process: initiation, the recognition of a general need for information; selection, the attempt to identify a general topic for searching; exploration, exploring the general topic; formulation, creating a focused idea of the topic; collection, the actual search for information; and presentation, the end of the search and preparation for reporting (Table 2.1).

Her model foregrounds the changing feelings, thoughts and actions that people experience across these stages. She emphasizes that such feelings are a normal and influential part of the search process, and that particular affective and cognitive states are related to stages in 
searching. This integration of affective (feelings), cognitive (thoughts) and physical (search actions) elements make this model unique (Table 2.1).

\begin{tabular}{|c|c|c|c|c|c|c|}
\hline \multirow[b]{3}{*}{$\begin{array}{l}\text { Feelings } \\
\text { common to each stage }\end{array}$} & \multicolumn{6}{|c|}{ Stages in Information Search Process (ISP) } \\
\hline & Initiation & Selection & Exploration & Formulation & n Collection & Presentation \\
\hline & Uncertainty & Optimism & $\begin{array}{l}\text { Confusion / } \\
\text { Frustration / } \\
\text { Doubt }\end{array}$ & Clarity & $\begin{array}{l}\text { Sense of direction / } \\
\text { confidence }\end{array}$ & $\begin{array}{l}\text { Relief / } \\
\text { Satisfaction or } \\
\text { disappointment }\end{array}$ \\
\hline $\begin{array}{l}\text { Thoughts } \\
\text { common to each stage }\end{array}$ & Vague & & & $\begin{array}{l}\text { Narrowed / } \\
\text { Clearer }\end{array}$ & Increased interest & Clearer or focused \\
\hline $\begin{array}{l}\text { Actions } \\
\text { common to each stage }\end{array}$ & $\begin{array}{l}\text { Seeking background } \\
\text { information }\end{array}$ & & $\begin{array}{l}\text { Seeking relevant } \\
\text { information }\end{array}$ & & $\begin{array}{l}\text { Seeking relevant or } \\
\text { focused information }\end{array}$ & \\
\hline
\end{tabular}

Table 2.1. Information search process (ISP). Adapted from Kuhlthau, 1991.

\subsubsection{Context in user-centered models}

A key point in user-centered models in information science is that information discovery is defined through interactions - interactions between users and systems, but also interactions between contexts and users.

Context is a complex and variously defined concept in information research. In 1997, Dervin proposed three approaches information researchers take to conceptualizing context, which still resonate today: viewing context as a container or backdrop for a phenomena, attempting to itemize context from a list of inexhaustible elements, or seeing context as a carrier of meaning (Dervin, 1997).

Agarwal builds on Dervin's work to propose that the varying descriptions of context exist on a spectrum between positivist, objective conceptualizations and more interpretivist, subjective views (2018). Positivist views, as described in (Dourish, 2004), depict context as being a list of nameable, stable elements that can be enumerated by a researcher; this view of context is common in the user-centered models reviewed above.

These models portray context as a list of primarily cognitive and affective factors influencing information activities (Courtright, 2007). In the stratified model, however, Saracevic not only names cognitive elements on the user side (i.e. tasks, intent and knowledge structures) but also highlights factors on the system side (indexing, software algorithms, etc.) that shape information-seeking interactions (1996). Ingwersen's model also discusses other aspects of context, recognizing the role of socio-organizational factors, including professional domain (1992, 1996). 
These user-centered models emphasize that context shapes a user's activities; with the exception of changes in a user's cognitive or affective states, they do not discuss how search activities affect context. The positivist view of context typical in these models is mirrored in their treatment of information. Although interactions such as indexing, representation and visualization may affect how a user interprets or interacts with content, information itself is seen as being an object which can be acted upon, aligning again with Buckland's view of information-as-thing (Buckland, 1991).

\subsubsection{The information journey}

The user-centered models reviewed in the previous section reveal important insights. Ingwersen's model, for example, suggests that cognitive structures are determined locally by a person's social experience. Saracevic's idea of adaptation suggests evolving relationships between systems and users. Belkin's model attempts to foreground search practices and interactions, rather than portraying a user and a system with fixed boundaries. Bates' berrypicking model highlights the changing nature of information needs and the distribution of information.

Taken together, these models characterize information-seeking as a dynamic process, defined through interactions, that involves multiple strategies over time; they also highlight different elements involved in discovering information. These models do not detail how information is used per se, although there is a recognition that the searcher's task shapes and is shaped by information-seeking. Similarly, these models do not detail the sensemaking processes that are inextricably linked with searching for information; sensemaking in information search is often taken up specifically in other work, notably (Dervin,1992; 1998; Russell et al., 1993; Pirolli \& Card, 2005).

Blandford and colleagues (Adams and Blandford, 2005; Blandford and Attfield, 2010) build on a series of their past studies to propose the idea of the information journey, which reflects many key insights from across research fields and information-seeking models (Figure 2.5), while including notions of sensemaking and use. 


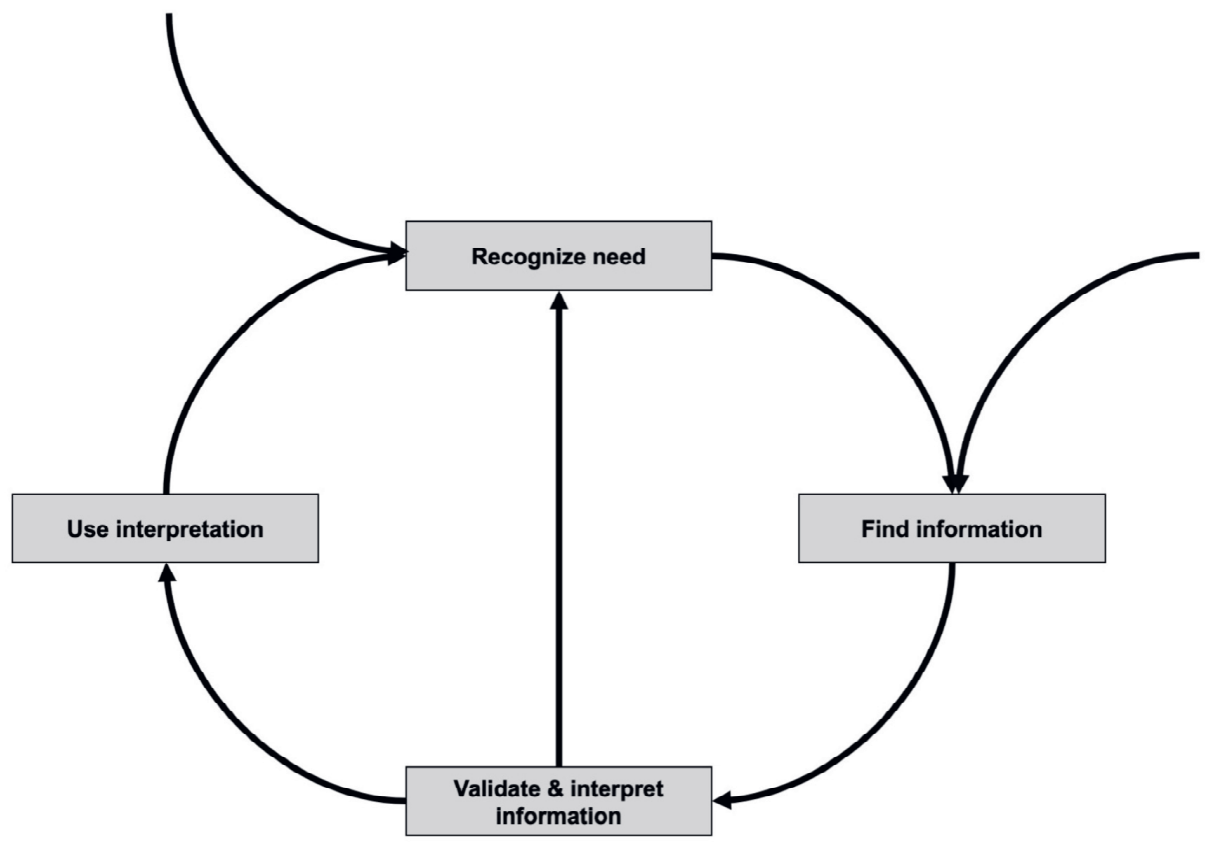

Figure 2.5. Information journey. Adapted from Blandford \& Attfield, 2010.

In this model, a searcher progresses through four stages: recognizing the need for information; acquiring information, either through active searching, serendipitous discovery or being told about it; interpreting and evaluating the information, and using the interpreted information. Information needs can be triggered by external factors and situations, as represented by the arrow at the top left of Figure 2.5. Although information needs may at times be clearly articulated, they may also be tacit, evolving or unrecognized (as represented by the arrow on the top right of Figure 2.5).

Information-seeking is seen as a cycle of interactions, shaped by these needs and by an individual's intended use(s) for the information. Although the arrows in the model imply an order of events, the information journey is not necessarily sequential; nor will an individual always enact all of the phases.

A slightly modified version of the information journey (Figure 2.6), adapted to reflect my research questions, provides the cornerstone of the integrated framework which I present in Section 2.5. In particular, this modified version highlights users and their needs and their practices of discovering, evaluating or making sense of and (re)using information, or in the case of my research questions, data. I make the users explicit in Figure 2.6, but otherwise, 
as in the original model, the focus remains on the practices involved in the information journey.

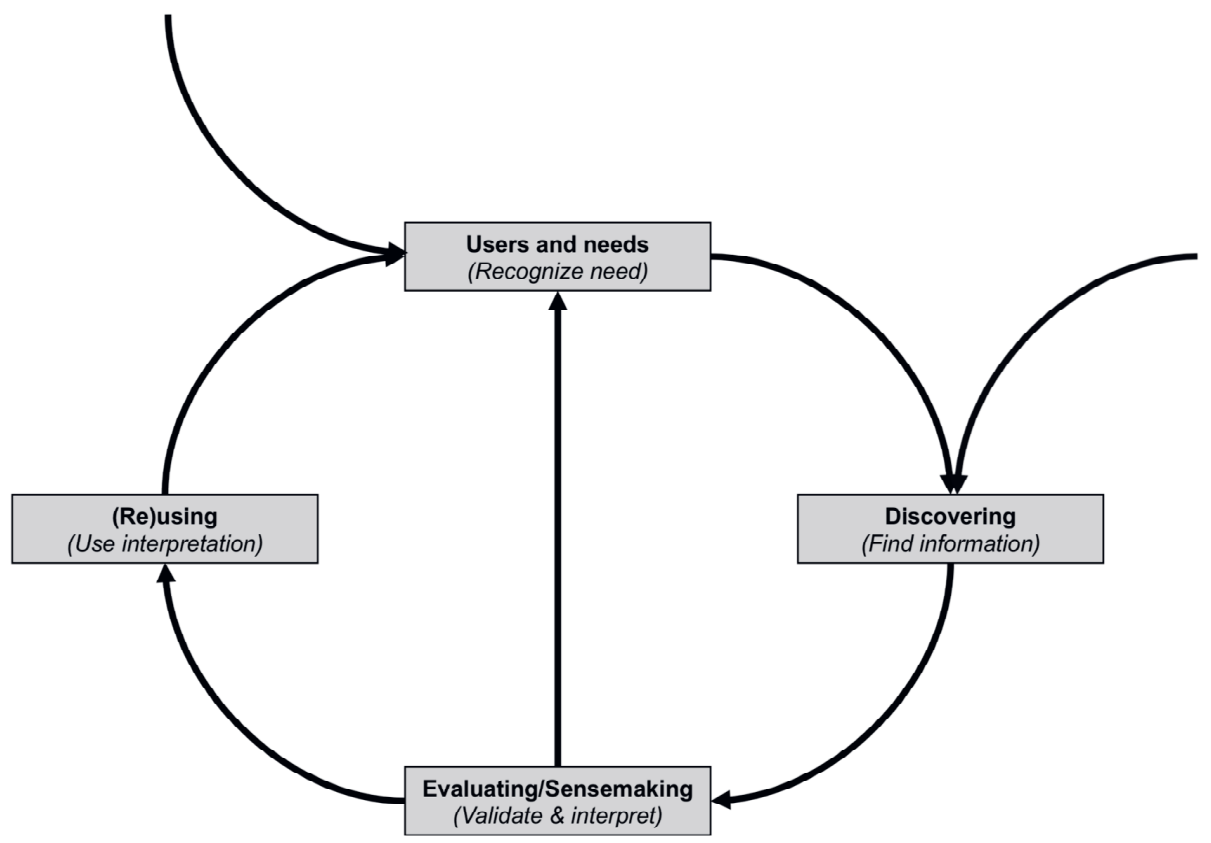

Figure 2.6. Modified version of the information journey model. Original names for the elements of the information journey indicated in italics.

\subsubsection{Limitations of user-centered models}

Van House summarizes many of the limitations of user-centered models (2004). Perhaps obviously, the user-centered models focus on the viewpoint of one individual acting in a particular role: the person looking for information. This person is defined in terms of his/her interactions with an information system; other roles that a person enacts, which may influence information practices, are not addressed. Individuals who do not interact with systems, non-users, are also not typically represented. Perhaps with the exception of the model proposed by Ingwersen, user-centered models do not meaningfully draw out other actors involved in information discovery processes; nor do they account for shared or distributed actions (Talja \& Hansen, 2006).

The idea of an information need, which undergirds these models, places the actor within a model of perceived lack. An individual may not have, or may not be aware of, particular needs. The information journey model described above recognizes that individuals may not always be aware of their information needs, and that discovery may occur serendipitously, addressing another criticism of many models - the assumption of goal-directed behavior. 
Perhaps most notably, the plethora of user-centered models and their pervasive use in information science creates a situation where the triad of users, needs and goal-directed behavior becomes "naturalized rather than understood as one possible model" (Van House, 2004, p.64).

\subsection{Focus on science $\&$ technology studies}

Science and technology studies offers many alternative approaches to the user-needs-goals structure common in models of information seeking. Here, I discuss two broad themes present in STS research, sociotechnical networks and an emphasis on studying practices, that surface in how STS has treated concepts important in information-seeking models, in particular users, communities and context.

\subsubsection{Sociotechnical networks and practices}

Much of STS embraces the idea of some form of "sociotechnical ensemble" (Bijker, 1995) or network (Law, 1992). Sociotechnical networks situate knowledge and technology within dynamic configurations of people, practices and materials, shaped by changing political, economic and cultural factors (van House, 2004). Elements in these networks are not inherently "social" or "technical." Rather, they are part of a seamless web where social elements are technically constructed and the technical are socially constructed (Bijker, 1993). In STS, these elements, as well as the study of practices themselves, cannot be analyzed in isolation, as they form part of an inseparable whole (Sismondo, 2004).

This reflects another core theme in STS, the principle of symmetry, which is grounded in the idea that both true and false beliefs should be analyzed using the same conceptual lens (Bloor, 1991/1976). In the context of sociotechnical networks, the idea of symmetry is extended to mean that no single element - human or non-human (Latour, 1983), working technology or non-working technology (Pinch \& Bijker, 1984) - is privileged over another. Each element is therefore of equal importance and can be analyzed in the same terms (Bijker, 1993).

This affords numerous entry points for studying sociotechnical networks. A particular "sociotechnology," i.e. a system of dikes in the Netherlands (Bijker, 1995) or a particular practice, i.e. showering (Hand et al., 2005), can be used as an entry to tracing the connections and relationships between different elements. In this way, the sociotechnical ensemble itself becomes the primary unit of analysis (Bijker, 1993). 
Studying practices provides an avenue to further examine relationships between the social, material and technical. Although the practice approach in STS is extremely diverse, studies converge on viewing practices as materially mediated arrays of activity centering around shared, embodied understandings (Schatzki, 2001). Focusing on practices can reveal how artefacts, people and technologies are brought together or fall apart (Shove, et al., 2012). Perhaps most importantly, foregrounding practices allows STS researchers to discover what people actually do, thus providing a way to counter often idealized accounts of technology use or scientific knowledge production (Knorr Cetina, 1999).

\subsubsection{Users}

"Users" are not a typical focus of research in STS, which has a history of examining the practices of scientists, engineers and technologists. When users of a technology are studied as such, they are examined reciprocally. Studies investigate how technology shapes user practices, but also how users shape the development of technologies (Oudshoorn \& Pinch, 2003).

One well-known approach that brings attention to users is the social construction of technology (SCOT), proposed by Bijker \& Pinch (Pinch \& Bijker, 1984; Bijker \& Pinch, 1987). This approach begins by identifying relevant and non-relevant social groups around a particular technology and describing their viewpoints. A key idea in SCOT, known as interpretive flexibility, is that different social groups will construct very different meanings for the same technology. There is therefore no single essential meaning or use which can be derived just from an artefact itself. Eventually, through a series of "closure mechanisms" the interpretive flexibility of a technology disappears and a technology stabilizes.

An example of how SCOT has been applied can be seen in the analysis of the development of the modern safety bicycle (Bijker \& Pinch, 1987). Early bicycles had a high-wheeled design, which was interpreted very differently by various social groups. Young men with money to burn, the first users of the bicycle, embraced the design, seeing the bicycle as a way to prove and test their daring. Nearly everyone else, particularly elderly men and women, saw the bicycle as an unsafe technology to be avoided. Eventually, the modern version of the bicycle stabilized and became dominant, as the design was accepted by the larger social group valuing safety over daring. The success of this technology was therefore not the result of a working design, but rather was the result of it being accepted by particular social groups. 
Others argue that users of a technology can be taken as an entry point into studying sociotechnical networks. Focusing on the "consumption junction," the point at which users choose between competing technologies, can help to understand the diffusion of technologies and identify their unintended uses or consequences (Cowan, 1987). Pushing back against the term "users," work in social informatics proposes the idea of "social actors," which recognizes that individuals using technologies are not primarily defined by that use, but rather enact multiple roles and inhabit multiple contexts where technologies are present (Lamb \& Kling, 2003).

Focusing only on the people who engage with a technology ignores the people who do not and reinforces the idea that technology use is the norm (Wyatt, 2003). As with users, nonusers are not a homogenous group. As Wyatt discusses in her analysis of non-users of the Internet, people may not have access to a technology, they may actively choose not to engage with it, or they may have tried it once or twice and decided that it was not for them. Users should therefore be "“'conceptualized along a continuum, with degrees and forms of participation that can change" (Wyatt, 2003, p. 77). Looking at the frequency and various types of use and non-use can help to contextualize the meaning of both, as well as potentially inform design (Wyatt, 2003).

The above work, as well as that presented in (Oudshoorn \& Pinch, 2003), emphasizes key findings about users within STS. Users are not passive recipients of technology, but are rather active participants in its development and adoption. Users' actions are not shaped by technology alone, but are a result of reciprocal relationships and are situated within dynamic social contexts. Both users and uses of technology are diverse and multiple. Varying social groups will see and use technologies in different, perhaps unexpected ways; this provides a motivation for studying practices of use - to understand how technologies are actually used or not used.

\subsubsection{Communities}

Much work in STS argues that the community or collective, rather than the individual, is the entity that "knows" (van House, 2004). Knowledge production is situated within these collectives, which have unique norms, practices and tools. The actions of an individual are representative of these collective ways of knowing and producing knowledge (Knorr Cetina, 1999); the study of individual actors is therefore inextricably linked with the study of communities. 
One often used framing for studying communities is Knorr Cetina's idea of epistemic cultures (Knorr Cetina, 1999). Epistemic cultures are not communities per se, but are defined as social and material arrangements and mechanisms that together constitute how knowledge is constructed within a given field. Knorr Cetina emphasizes that these groups are not equivalent to disciplinary domains, but can be best conceived of as "machineries of knowing composed of practices" (p.10).

The key to unravelling how these machines operate is to study practices. Knorr Cetina builds on her studies in two settings, high energy physics and molecular biology, to both illustrate the idea of epistemic cultures and to highlight the diversity which exists within and between them. To do this, she compares the highly theoretical, communitarian practices of physics, with its shared resources and practice of hyper-authorship, to the more individual, experiential practices in biology. Scientists themselves are enfolded within and are derivative of the conventions, devices and ways of thinking present in these different epistemic cultures.

Another oft-used framing is that of communities of practice (Lave \& Wenger, 1991; Wenger, 1998), which, at a simple level, can be defined as relations between people who engage in an activity together (see Bowker and Star, 1999). Communities of practice (CoP) are defined by a common enterprise, a shared repertoire of activities and understandings, and processes of mutual engagement, in which people negotiate the meaning of practice together.

Learning is a critical part of becoming a member of a community of practice, which are intrinsically dynamic groups. An individual needs to first learn the skills and meanings of a particular practice before becoming a member, through a process termed "legitimate peripheral participation." It takes even more effort to become an established member of a community, although not everyone who gains membership will choose to remain.

Bowker \& Star also emphasize that membership in a CoP is mediated not only by shared understandings and activities, but also by the co-use of objects and tools (Bowker \& Starr, 1999). They claim that most people are members of multiple communities of practice, and that becoming a member of certain CoPs may be an easier and speedier practice than for others. Learning to play the violin in an orchestra, for example, requires extensive lessons, shared exercises and personal practice; learning to play croquet with one's neighbors will most likely require less time and effort. 
Both communities of practice and epistemic cultures illustrate core factors of how STS conceptualizes communities. Communities are dynamic groups of individuals that are forged through common practices, epistemic norms, and shared objects. Disciplinary domains alone do not define communities. Individuals can belong to multiple communities, and individual actions are shaped by communities themselves.

\subsubsection{Context in information research}

As can be seen from the discussion thus far, STS involves an inherently contextual research approach. Knowledge, communities, users and technology are situated within and constructed by dynamic contexts. I focus here on how similar views of context are present within information research.

STS views of context correspond to what Agarwal terms subjective or interpretivist views (see Section 2.3.3; Agarwal, 2018), and Dervin's conceptualization of context as a carrier of meaning (1997). In these views, context is portrayed as a set of relationships between actors, objects and activities that is enacted and local to each activity (Dourish, 2004). As Dourish summarizes, "context isn't described as a setting; it is something that people do" (Dourish, 2004, p.22).The boundaries of context are therefore seen as evolving, local and dynamic.

As Courtright (2007) notes, such views of context are often discussed using alternative framings, such as information worlds or environments (i.e. Lievrouw, 2001), information ecologies (Nardi \& O'Day, 1999) or information grounds (Pettigrew, 1999). Lievrouw, for example, proposes that information environments are the result of interactions between two evolving aspects: institutional practices and personal/relational practices. Institutions produce, broker and distribute information while at the same time, individuals engage in smaller-scale, interpersonal information creation and sharing. These two aspects, which differ by place or group, are either facilitated or hindered by information technologies. Again, technology is seen as both shaping and being shaped by the practices which compose an information environment, and it is possible for an individual to inhabit multiple, overlapping contexts at the same time (Lievrouw \& Farb, 2003).

Although context is often framed in information research from the point of view of an individual actor, this is not always the case. Talja and colleagues examine how information researchers themselves contribute to context (1999). Drawing on Dervin's contentions that the knower and the known are bound together and that context cannot be conceived of as a single entity (1997), Talja et al. define context as the crossroads between researchers and 
the data (about information practices) which they are analyzing. Agarwal also recognizes a "shared" or collective view of context, where context is created from the viewpoint of individuals with shared identities (Agarwal, 2018). This resonates with other work, such as Chatman's theory of "life in the round" (Chatman, 1999), developed through the study of underserved populations, which sees social communities as bounding information practices.

Agarwal reaches twelve conclusions about how context is represented in the broader information research literature (Agarwal, 2018). I highlight three of these conclusions which are especially resonant with concepts in STS. First, context is given meaning through practice, as it is locally defined at the point of interaction between actors and objects. Secondly, context is always about a relationship between actors and external objects, thoughts, feelings, colleagues, and tasks. These elements do not determine context on their own, but are rather held together through relationships. Finally, context looks different from varying perspectives; an information researcher's view of context will be different from a user's perception which will be different from a system developer's view.

\subsubsection{Unifying concepts}

Through the above analysis of users, communities and context, the core STS tenets of sociotechnical networks, symmetry and practice-oriented approaches are evident. Other common themes, related to these core ideas, also surface.

The first is the importance of examining objects of research from different perspectives. This becomes clear in Wyatt's analysis of non-users to further contextualize the concept of users and use; in SCOT's approach to describing the viewpoints of varying social groups surrounding a technology; and in Knorr Cetina's comparative analysis of high energy physics and molecular biology to understand epistemic cultures. It also arises in understanding context as something that is constructed differently from different viewpoints, such as that of individual actors, communities or information researchers.

Secondly, the diversity and multiplicity woven throughout science and technology becomes visible. Users and non-users are not homogenous groups; nor are communities defined by a single characteristic, such as disciplinary domain. Different social groups construct a variety of meanings and uses for technologies. Context and communities are formed in diverse ways, as people, technologies and information are brought together in local configurations. This diversity and multiplicity provides a driving motivation for studying practices. 
Finally, users, communities and context are reciprocally shaped by dynamic elements in relation with each other. Users are not passive recipients of technology, but actively participate in its construction and adoption. Communities are forged through dynamic processes of learning, where individuals gain membership, establish themselves, or leave. Contexts and communities are defined through practices, which are situated within seamless webs of epistemic norms, objects and technologies.

\subsection{Integrated theoretical framework}

This chapter has thus far presented the foundations for the theoretical framework used in this dissertation. It has examined user-centered models for information-seeking, rooted in information behavior and information retrieval, and explored how STS has approached key elements of these models - users, communities and context - drawing out common concepts across this work (Section 2.4.5). This final section brings these two perspectives together to structure and frame the research presented in the remainder of this thesis.

The user-centered models reviewed in Section 2.3.2 portray information-seeking as an evolving process defined through interactions between users, systems and context. They emphasize the activities undertaken by users to discover information, highlighting the dynamic nature of information needs, as well as an individual's cognitive and affective structures. A slightly modified version of the information journey model (Figure 2.6) structures my research, where I take users and their data needs as an entry point to investigating practices of discovering, making sense of, and using data in research.

The analysis of users, communities and contexts in STS presented in Section 2.4 provides an avenue for expanding the information journey model. Users are not atomic individuals, but are rather social actors enfolded in epistemic communities who engage with technologies in various ways. Contexts are not enumerable stable entities, but are instead dynamic and enacted. Examining objects of research from different perspectives helps to uncover actual practices of use, to reveal the diversity and multiplicity of practice and to explore relationships between dynamic, sociotechnical elements (Section 2.4.5).

A series of guiding questions, when considered together, provides a way to tease out these unifying concepts drawn from STS (Section 2.4.5), in relation to practices of data discovery (Box 2.1). Identifying the various communities, types of data and technologies used is an important step in understanding data discovery practices. This involves asking "which" 
questions, i.e. "which communities" or "which data;" it also involves paying attention to which entities are not being taken up or which communities are not engaging in a practice (Q1).

(Q1) Which (people, roles, communities, data, technologies, etc.) / which not?

(Q2) What are people actually doing? How are these practices forming, changing or stabilizing?

(Q3) For which purposes and to which effects?

(Q4) How are practices related to other elements (social, technical, material, etc.) and to other practices?

Box 2.1 Guiding questions to draw out STS perspectives

Studying practices requires observing what people are doing, as well as questioning how these practices are changing or stabilizing in relation to technologies, materialities and norms (Q2). Understanding the motivations behind and consequences of practice helps to provide context as well as to trace relationships between particular practices and other elements (Q3; Q4). Repeatedly asking these questions is likely to expose new connections and interdependencies, leading to deeper, more diverse representations and understandings.

Figure 2.7 brings together and visualizes these two perspectives, the modified version of the information journey model and the questions posed in Box 2.1 which draw out the identified guiding concepts from STS. The figure explicitly situates both perspectives within a web of other sociotechnical elements, including, but not limited to, data, search systems, communities and other practices of (re)search. 


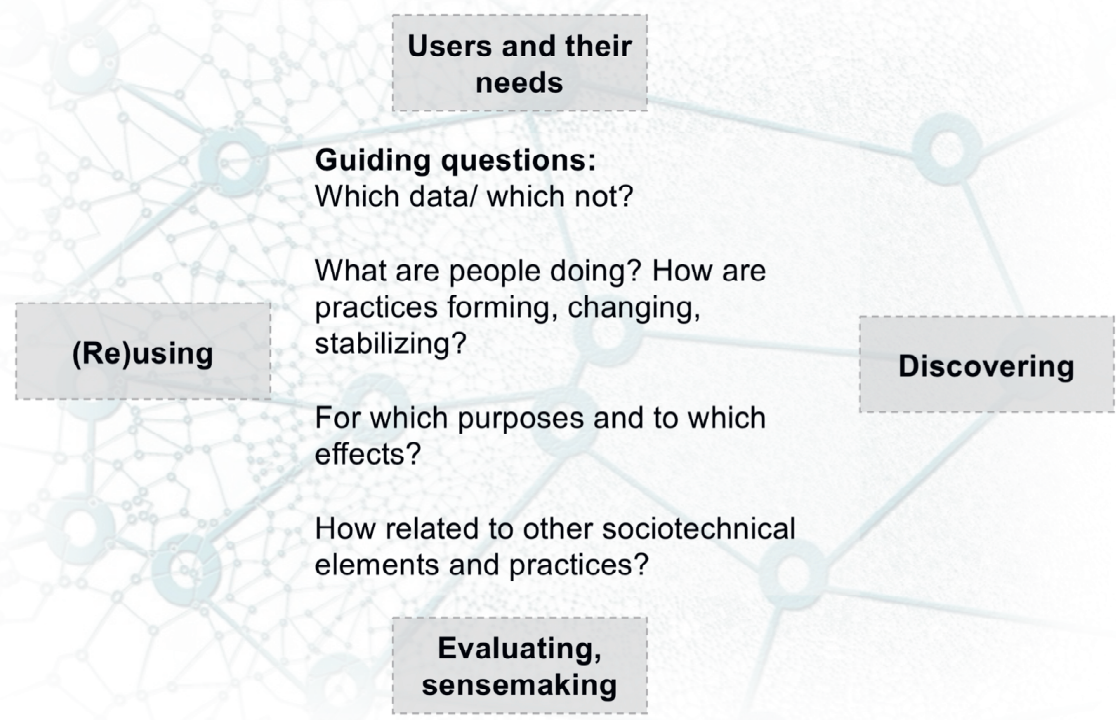

Figure 2.7. Visualization of theoretical framework

As shown throughout this chapter, the ideas represented in this figure are well-established in information science and science \& technology studies. I bring these ideas together in a new way, using guiding concepts drawn from STS to engage with a user-centered paradigm of data discovery. Using this approach, I build on insights from across areas of information research, while also looking for alternative perspectives, explanations and relationships. This allows me to explore data discovery as a situated practice while still using a framework that is translatable to systems development and design.

This theoretical framework structures the research presented in Chapters 3-5. These chapters are organized into sections roughly corresponding to the components of the modified information journey model; STS-inspired perspectives are drawn out in the analysis, presentation of findings and discussions. Chapter 6 , which describes the data collected from the survey study, does not use the framework. Chapter 7 , the result of a collaboration with researchers in human computer interaction, hones in on a particular practice implicated in the framework - data-centric sensemaking. As the focus of this chapter is not on data discovery as a whole, it does not follow the same structure of the other chapters, although it does draw on some of the ideas presented here.

The following chapter begins the presentation of empirical work by examining how the data discovery practices of a particular "community," individuals using observational data within 
selected disciplines, have been documented in the literature. This chapter employs an early iteration of the above theoretical approach, applying instead two separate frameworks - one drawing on work in interactive information retrieval and one rooted in STS-inspired work on data communities - to analyze the literature.

The chapter begins with the stated aim of identifying commonalities in practice to support design through the creation of a new model for data retrieval. It concludes, however, that diversity is in fact a common theme, and suggests that perspectives rooted in information retrieval alone are insufficient for understanding data discovery practices. 


\section{CHAPTER 3}




\section{Searching data: A review of observational data retrieval practices in selected disciplines}

Originally published as: Gregory, K., Groth, P., Cousijn, H., Scharnhorst, A., \& Wyatt, S. (2019). Searching data: A review of observational data retrieval practices in selected disciplines. Journal of the Association for Information Science and Technology, 70, 419-432.

https://doi.org/10.1002/asi.24165 


\title{
54 | Chapter 3
}

\begin{abstract}
A cross-disciplinary examination of the user behaviors involved in seeking and evaluating data is surprisingly absent from the research data discussion. This review explores the data retrieval literature to identify commonalities in how users search for and evaluate observational research data in selected disciplines. Two analytical frameworks, rooted in information retrieval and science and technology studies, are used to identify key similarities in practices as a first step toward developing a model describing data retrieval.
\end{abstract}




\subsection{Introduction}

Open research data is touted as having the potential to transform science and fast-track the development of new knowledge (Gray, 2009). In order for data to fulfil this potential, users must first be able to find the data that they need. This is not a simple task. Facilitating data discovery relies on developing underlying infrastructures, support systems, and data supplies (Borgman, 2015a). It is equally important to understand the behaviors involved in data retrieval, but a user-focused, cross-disciplinary analysis of data retrieval practices is lacking. This review explores the existing data retrieval literature and identifies commonalities in documented practices among users of observational data as a first step towards creating a model describing how users search for and evaluate research data.

Although information retrieval (IR) has been extensively studied for over sixty years (Sanderson \& Croft, 2012), data retrieval is a nascent field. Recent studies surrounding the issue examine how data are made available via data sharing (Tenopir et al., 2011, 2015), how researchers reuse data (Faniel et al., 2016; Pasquetto et al., 2017), and how systems are designed to optimize data discoverability and retrieval (Pallickara et al., 2012). Information documenting data retrieval behaviors is buried throughout other disciplinary and data-related literature and is not easy to identify (Gregory, Cousijn, et al., 2018).

We draw on work in information retrieval and science and technology studies (STS) to guide the identification of this buried literature and to develop our analysis frameworks. The first framework is based on established models of interactive information retrieval; the second framework builds on STS-inflected work examining data practices and communities. We begin by discussing the frameworks in more detail before using them to present and synthesize the data retrieval behaviors documented in the collected literature. We end with a discussion of commonalities across disciplinary communities and identify gaps in the literature and areas for future work.

\subsection{Analytical frameworks}

\subsubsection{Framework \#1: A broad view of interactive information retrieval} Information retrieval is an interactive process, involving a dynamic interplay between users and IR systems (Xie, 2008). Numerous models describe user-oriented interactive IR. Three of the most pivotal are Ingwersen's cognitive model (Ingwersen, 1992, 1996), Belkin's episode model (Belkin, 1993, 1996), and Saracevic's stratified interaction model (Saracevic, 
1996, 1997). Detailed characterizations of the strategies (e.g. Bates, 1990) and cognitive and affective stages in user-oriented information seeking (Kuhlthau, 1991) have also been proposed. Despite their differences, established models assume that users are actively involved in the search process and that context influences search behaviors (Rieh \& Xie, 2006; Xie, 2008).

Interactive IR models share a few key stages $^{3}$ (Wolfram, 2015) that are used to structure the first framework and to provide the main divisions of this paper:

- Users and Needs: describes user contexts and data needs

- User Actions: describes the sources and search strategies used to locate research data

- Evaluation: describes criteria and processes used when evaluating data for reuse

The term data retrieval is used in this review to refer to this entire complement of needs, actions, and evaluation behaviors.

\subsubsection{Framework \#2: A broad view of data communities}

Data practices can define communities in different ways (Birnholtz \& Bietz, 2003). Data communities form around disciplinary domains, (Faniel, Kansa, et al., 2013; Palmer et al., 2004), research approaches and data collection methodologies (Birnholtz \& Bietz, 2003; Weller \& Monroe-Gulick, 2014), and particular data sources (Brown, 2003; Sands et al., 2012). Both macro-level characteristics, such as using quantitative vs qualitative data (Birnholtz \& Bietz, 2003) and micro-level characteristics, such as participation in a specific research project, (Borgman, Wallis, \& Enyedy, 2007) can define community membership. A researcher may belong to multiple data communities simultaneously, or she may choose to define her community in unique ways (Birnholtz \& Bietz, 2003).

Here, we embrace a broad approach to conceptualizing data communities. The overarching data community used in this framework is based on accepted classifications of research data. While classifying data is a notoriously difficult task (Borgman, 2015a), broad categories that have proven to be useful are observational, experimental, or computational data (National Science Board, 2005; National Science Foundation, 2007). As a first step in testing

\footnotetext{
${ }^{3}$ IR systems are also an important part of these models. The first version of the article preprint (arXiv:1707.06937) includes an additional review of data retrieval systems.
} 
the validity of this conception of data communities, we focus on a community bounded by the use of a particular data type: observational data.

Observational data result from recognizing, recording, or noting occurrences. They are often produced with the help of instruments, and include weather observations, polling data, photographs, maps, and economic indicators (Borgman, 2015a; National Science Board, 2005). Observational data are used across disciplines; we therefore introduce disciplinary communities into the second framework to provide another level of analysis.

Science and technology studies research explores the role of disciplinary norms and behaviors in data practices (e.g. Leonelli, 2016). Subdisciplines and individual research groups may have unique data practices, different than those of the broader disciplinary community (Gregory, Khalsa, et al., 2018); while these differences are important, we suggest that commonalities are also important. In order to identify possible commonalities, we group the disciplines represented in the retrieved literature into five broad domains: astronomy, earth and environmental sciences (EES), biomedicine, field archaeology and social sciences.

This review centers on the role of the researcher as data user. While the discussion of data communities often takes the perspective of data producers, researchers play multiple roles, often mixing data production and consumption (Borgman, Van de Sompel, et al., 2015). We focus on consumers/users of observational data who use data they did not create either for new purposes and/or to support existing projects.

\subsubsection{Purpose of the frameworks}

Many studies employ case studies, interviews and ethnographic research to depict particular data practices in fine detail (Cragin et al., 2011; Weber et al., 2012) and are spread across disciplinary domains. While these studies provide great depth, it is challenging to bring them together in meaningful ways to identify similarities (Faniel, Barrera-Gomez, et al., 2013). The primary goal of this review is to use the macroscopic perspectives of the frameworks introduced above to identify commonalities in reported practices. Such a broad approach comes with two drawbacks: the loss of some of the complexity and detail of the original studies and a bias in the disciplinary scope.

Each section begins with a table synthesizing the reviewed literature through the lens of both frameworks. We then present the literature used to create these syntheses, structuring the 
findings by disciplinary community. In the discussion, we summarize and discuss the key findings from each section and identify common themes.

\subsection{Methodology}

Our literature collection methodology was informed by the first framework. We performed keyword searches related to information retrieval (e.g. user behavior, information seeking) and data practices (e.g. data sharing, data reuse, research practices) across all fields, primarily in the Scopus database. We also performed searches related to data search and data discovery and used bibliometric techniques such as citation chaining and related records. ${ }^{4}$

We closely read the nearly 400 retrieved documents to identify papers referring to observational data. As we read, we again applied the first framework, seeking descriptions of data users and their needs, sources and strategies used to locate data, and the criteria used to evaluate data for potential reuse. Few studies examine data retrieval practices directly; much of the information is buried within investigations of data sharing and data reuse or found in user studies of particular repositories.

\subsection{Findings}

\subsubsection{Users and needs}

In this section we analyze the diversity of users' data needs within the context of disciplinary communities. We adopt the characterization of background uses of data which support research and foreground uses which drive new research (Wynholds et al., 2012).

\footnotetext{
${ }^{4}$ For a detailed methodology and machine-readable bibliography, including references regarding data retrieval systems, see: https://doi.org/10.17026/dans-zgu-qfpi
} 


\begin{tabular}{|c|c|c|}
\hline $\begin{array}{l}\text { Users in this } \\
\text { community... }\end{array}$ & Need this type of data & $\begin{array}{l}\text { For these purposes } \\
\text { (italicized = foreground, normal = background) }\end{array}$ \\
\hline Astronomy & $\begin{array}{l}\text { Data from sky surveys, telescopes, archives, } \\
\text { repositories, data catalogs, virtual observatory } \\
\text { systems }\end{array}$ & $\begin{array}{l}\text { New questions of old data, baselines, instrument } \\
\text { calibration, physical properties, model inputs, data } \\
\text { integration }\end{array}$ \\
\hline $\begin{array}{l}\text { Earth \& Environmental } \\
\text { Sciences }\end{array}$ & $\begin{array}{l}\text { Plant, animal, water, weather, solar observations; } \\
\text { soil analyses, rock thin-section and satellite images; } \\
\text { maps, geographic, demographic and census data; } \\
\text { continuously collected and transmitted data, data at } \\
\text { temporal/spatial scales, raw and summarized data }\end{array}$ & $\begin{array}{l}\text { New questions of old data, meta-analyses, } \\
\text { calibration, context, baselines, reference, model } \\
\text { inputs, verification, comparison, environmental } \\
\text { planning, policy- and decision making, education, } \\
\text { instrument monitoring; data integration }\end{array}$ \\
\hline Biomedicine & $\begin{array}{l}\text { Images, complete fMRI studies, pathology results, } \\
\text { patient observations and demographics; population- } \\
\text { level disease data, behavioral data }\end{array}$ & $\begin{array}{l}\text { Disease/disorder research, new visualizations, } \\
\text { evaluations, } 3-D \text { anatomical pictures, preparing } \\
\text { research outputs, education, patient care }\end{array}$ \\
\hline Field Archaeology & $\begin{array}{l}\text { Field notebooks, photographs, artefacts, } \\
\text { stratigraphic baselines; data at temporal/spatial } \\
\text { scales }\end{array}$ & $\begin{array}{l}\text { New insights from data aggregation, comparison, } \\
\text { triangulation; training, dissertations, assignments, } \\
\text { preparing tours, inventories of local excavations }\end{array}$ \\
\hline Social Sciences & $\begin{array}{l}\text { Survey data (often only one question is of interest), } \\
\text { long-running datasets/surveys, interviews, archival } \\
\text { documents, images, videos }\end{array}$ & $\begin{array}{l}\text { Re-interpret datasets; new questions, comparative } \\
\text { research, comparison, preparations, training, } \\
\text { dissertations }\end{array}$ \\
\hline
\end{tabular}

Table 3.1. Users' observational data needs by disciplinary community.

\section{Astronomy}

Much astronomical research can be classified as big science, involving large international projects supported by extensive knowledge sharing infrastructures (Borgman, Wallis, \& Enyedy, 2007). Big science is not the only approach, as astronomers also conduct research falling within the long tail of science (Wynholds et al., 2011). Access to the vast amount of available research data is remarkably open, and data sharing is generally encouraged (Hoeppe, 2014; Pepe et al., 2014).

\section{Data needed.}

Data from large-scale sky surveys, such as the Sloan Digital Sky Survey (SDSS), form the foundation for many research projects (Pepe et al., 2014). Similarly, the data practices of researchers working with the SDSS are the cornerstone of the data retrieval literature in astronomy (Borgman, Darch, et al., 2016; Sands et al., 2012; Wynholds et al., 2011).

Sky survey data fuel studies involving further data processing; derived data are then used as the basis for publications (Pepe et al., 2014). Direct data from ground- and space-based telescopes, data located in data repositories and catalogs, and data found through federated queries of virtual observatory systems are important sources (Sands et al., 2012; Wynholds et al., 2012). Theoretical researchers also use observational data from established archives as model inputs (Sands et al., 2012). 


\section{Data uses.}

Astronomers combine multiple datasets, often from multiple archives or telescope types, during a single project (Sands et al., 2012; Wynholds et al., 2011). Merging data about the same target from different instruments poses a significant challenge (Hoeppe, 2014; Zinzi at al., 2016).

Astronomers use external data for foreground purposes driving new scientific inquiries and leading to new discoveries (Wynholds et al., 2012; Wynholds et al., 2011), and for background purposes supporting research, such as study baselines, calibrating instruments, and searching for specific physical properties (Wynholds et al., 2012).

\section{Earth and environmental sciences}

A variety of disciplines and subdisciplines are represented in the literature at differing levels of granularity. Data retrieval practices are sparsely documented in fields such as volcanology, but discussions are increasing in other disciplines, i.e. the water sciences (e.g. Dow et al., 2015). This is partly due to a change in data collection techniques. As researchers transition from primarily manual field work to using sensors enabling continuous collection, they must find new ways to manage their data (Maier et al., 2012). The ecologists involved in the multidisciplinary Center for Embedded Networked Sensing (CENS) are an example of researchers caught in this transition (see Borgman, Wallis, \& Enyedy, 2007; Wallis et al., 2013).

\section{Data needed.}

Biodiversity researchers require an incredible multiplicity of data. Potentially any information about life on earth, from satellite photos to forest inventories, could be important (Bowker, $2000 \mathrm{~b})$. Scientists need information about species distribution and occurrence, population trends, and geographic raw data (Davis et al., 2014). The needs of CENS researchers exemplify what Bowker terms "data diversity," as they use weather, solar, and river observations, as well as remote sensing and demographic data (Bowker, 2000a; Wallis, et al., 2013). Data diversity is also the norm in the geo- and water sciences. Volcanologists rely on images of thin rock sections, chemical analyses and characterizations of the earth's crust. Additionally, stratigraphers use astronomical observations and numerical data extracted from graphs to study geologic history (Weber et al., 2012). Geographers need data spanning the physical and social sciences, requiring topographic, geologic and demographic maps, satellite images and drawings, and census data (Borgman et al., 2005). Water scientists need streamflow, evaporation, groundwater level, and water quality measurements (Beran et 
al., 2009). Although they do not exist for every condition, continuously collected data that can be analyzed by location and time are expected (Dow et al., 2015).

This need for data at different geographic and temporal scales connects the disciplines. Atmospheric scientists need large amounts of observational data from specific regions and times for their models (Pallickara et al., 2012). Data collected at local levels can be more important than data collected at national or state levels, as shown by a user survey from (Davis et al., 2014).

The Davis et al. survey is one of the few that differentiates between the data needs of different types of users; another example is a study at the Center for Coastal Margin Observation and Prediction (CMOP) (Maier et al., 2012). Internal and external researchers using CMOP data want succinct data overviews. Policy and decision makers need thematic collections summarized on one page, with salient data clearly marked; users in education sectors are also interested in CMOP data, although their specific needs have not yet been studied (Maier et al., 2012).

Like researchers, environmental policy and decision makers need information from different locations and times, but they have difficulties accessing the information (McNie, 2007) or finding the right type. Data produced by scientists are not automatically useful for policy makers (Cash et al., 2003). Environmental planners, i.e., may not need the same depth of information as researchers (Van House et al., 1998); reflecting this, differentiated data products for diverse users are being explored (see Baker et al., 2015).

\section{Data uses.}

CENS researchers use external data solely for background purposes, such as contextualizing their own data and calibrating instruments (Wallis et al., 2013; Wynholds et al., 2012). Other background uses include benchmarking and as references (Bowker, 2000 b). Some ecologists do reuse external data to answer new questions (Zimmerman, 2007) or to create meta-analyses (Michener, 2015).

Integrating diverse data is problematic across the environmental sciences. Data collected at different scales and using different nomenclatures are difficult to merge (Dow et al., 2015; Maier, et al., 2014; Bowker, 2000b). Natural variances in systems and populations further complicate fitting biodiversity data together (Bowker, 2000b; Zimmerman, 2007).

Stratigraphers use one dataset to calibrate another as they construct geologic timelines used as baseline data by other researchers (Weber et al., 2012). Atmospheric scientists and 
climatologists grapple with problems stemming from metadata variation (Pallickara et al., 2012) and differences in community data practices (Edwards et al., 2011).

Modelers use external data at specific points in the research process. After reformatting and regridding data to fit model specifications, earth scientists use observational data to initially force models and for parameterization; data availability limits the types of studies undertaken (Parsons, 2011). Coastal modelers engage in similar behavior, continually calibrating and benchmarking their models, and comparing outputs to external observational data (Maier et al., 2012; Weber et al., 2012).

Environmental planners use data not only to make decisions, but also to defend their viewpoints, to persuade, and in education. (Van House et al., 1998). Although detailed studies of non-scientists' data needs are lacking (Faniel \& Zimmerman, 2011), reported "background uses" of oceanographic data include preparation for triathlons, search and rescue operations or fishing expeditions (Weber et al., 2012)

\section{Biomedicine}

The biomedical literature focuses on fields centering on imaging, such as neuroscience and radiology.

\section{Data needed.}

As neuroscience embraces big science methodologies, the field is struggling with how to make data available, discoverable, and usable (Choudhury et al., 2014). Researchers rely on visualizations of normal and abnormal brains, although they also consult brain bank samples (Beaulieu, 2004). Sometimes researchers need raw fMRI studies, including detailed metadata; sometimes images and scans suffice (Key Perspectives, 2010; Van Horn \& Gazzaniga, 2013). Neuroimaging data are complex, consisting of numerous brain section slices, time-points, and other variables (Honor et al., 2016). Neuroscientists incorporate more than just imaging into their work, using demographic, genetic, and behavioral data (Williams et al., 2009).

Clinicians and medical researchers also use a mixture of images and other observational data, such as pathology results, clinical data (e.g. progression of tumor grades), patient demographics, and population-level disease data (Kim \& Gilbertson, 2007). Medical images are an essential part of workflows in fields such as radiology (Markonis et al., 2012), where health care professionals tend to search for two types of images: general medical images (e.g. images of anatomic organs) and specific medical images, which are used for clinical or 
comparison purposes (Sedghi et al., 2011). Users need images collected with different modalities (X-rays, CT scans, and MRIs) (Kim \& Gilbertson, 2007); medical students need images corresponding to their current courses (Müller et al., 2006). All reusable medical data must be provided in a way protecting patient privacy (Erinjeri et al., 2009).

\section{Data uses.}

Neuroscientists use imaging data for comparisons, evaluations, and creating threedimensional pictures of brain anatomy (Beaulieu, 2004). A single scan is of little value unless incorporated into a larger database of scans. Aggregating individual scans creates complete virtual brains that can be manipulated to facilitate new discoveries (Beaulieu, 2004), as in the case of combining fMRI scans from different populations to yield insights about Alzheimer's biomarkers. (Van Horn \& Gazzaniga, 2013).

In a study of clinicians, researchers, educators, librarians, and students, users incorporate images in research, patient care and education (Hersh et al., 2005). A follow-up study further characterizes these needs, showing that images are used for self-education; educating medical students, patient education, making difficult diagnoses, and developing research ideas, grant proposals, and publications (Kalpathy-Cramer et al., 2015).

\section{Field archaeology}

Archaeology is another field in transition. Methodologies and data practices are changing, as data move away from being published in analog-only formats to being made available in digital repositories (e.g. Arbuckle et al., 2014); this facilitates data aggregation to study phenomena such as domestic livestock expansion (Arbuckle et al., 2014; Atici et al., 2017). Interdisciplinarity and data diversity are thriving in archaeology, as research projects can involve soil scientists, zooarchaeologists, and material scientists (Faniel, Kansa, et al., 2013)

Metadata and documentation of methods and site conditions are extremely important in archaeology, as original sites are often "decomposed" during the research process (Faniel, Kansa, et al., 2013). Data recording and metadata standards do not exist (Faniel \& Yakel, 2017); making integration across contexts and collection methodologies challenging (Niccolucci \& Richards, 2013; Faniel \& Yakel, 2017).

Field archaeologists need field notes, photographs, and artefacts in museum collections (Faniel, Kansa, et al., 2013). Geographic, stratigraphic and chronological baseline data are also vital (Atici et al., 2013). Archaeologists compare finds from the field to museum collections, often triangulating data from multiple sources (Faniel, Kansa, et al., 2013). 


\section{$64 \mid$ Chapter 3}

Researchers are not the only "consumers" of archaeological data; students, hobbyists, and employees of museums and companies use data for diverse background and fewer foreground purposes, e.g. aggregating discrete units of "raw data" (Borgman et al., 2018).

\section{Social sciences}

Reusing quantitative data in the social sciences is well-established (Kriesberg et al., 2013; Faniel \& Yakel, 2017); the reuse of qualitative data is complicated by issues of participant confidentiality and the embeddedness of the researcher in data creation (Broom et al., 2009).

Social scientists need data from surveys and long-running datasets (Shen, 2007).

Researchers are often interested in only one data point or survey question. Details about the operationalized variables or measured constructs usually are not present when examining individual questions in isolation (Dulisch et al., 2015). Social scientists also need archival documents, images, videos, and interview data (Karcher et al., 2016).

Data can be reused for comparative research or to ask new questions, reinterpret datasets, or verify findings (Corti, 2007). Background uses, i.e. preparing for data collection, are common (Parry \& Mauthner, 2005).

Kriesberg and colleagues examine the needs of early career researchers (ECRs) in quantitative social sciences, archaeology and zoology. External data are used in training and dissertations; young researchers may reuse data more often, due to difficulties collecting their own data (2013).

\subsubsection{User actions}

This section examines the resources and strategies used within different communities to locate data. 


\begin{tabular}{|c|c|c|}
\hline $\begin{array}{l}\text { Users in this } \\
\text { community... }\end{array}$ & Use these resources & In this way \\
\hline Astronomy & $\begin{array}{l}\text { NASA archives, journals, personal exchanges, } \\
\text { personal websites, general search engines }\end{array}$ & $\begin{array}{l}\text { Querying archives, extracting data from articles into } \\
\text { new tables, informal personal requests }\end{array}$ \\
\hline $\begin{array}{l}\text { Earth \& Environmental } \\
\text { Sciences }\end{array}$ & $\begin{array}{l}\text { Journals, personal exchanges, repositories, } \\
\text { databases, natural history collections, general } \\
\text { search engines, industry }\end{array}$ & $\begin{array}{l}\text { Extracting data from articles, email/ } \\
\text { telephone/letters, metadata searches, faceted } \\
\text { searching, filtering, aggregating data to create new } \\
\text { datasets, "bounded" strategies (by journal, location, } \\
\text { time) }\end{array}$ \\
\hline Biomedicine & $\begin{array}{l}\text { Online image repositories, local image and patient } \\
\text { information systems, personal image collections, } \\
\text { Google Images, journals }\end{array}$ & $\begin{array}{l}\text { Local systems - patient name/identifier; Online } \\
\text { sources - keyword and hierarchical searches, short } \\
\text { queries for images }\end{array}$ \\
\hline Field Archaeology & $\begin{array}{l}\text { Personal connections - museum staff and data } \\
\text { producers, natural history collections, museums, } \\
\text { repositories/archives, publications }\end{array}$ & $\begin{array}{l}\text { Searching by location (keywords, browsing), } \\
\text { collaborations to gain additional data }\end{array}$ \\
\hline Social Sciences & $\begin{array}{l}\text { Survey banks, data catalogs (i.e. DBK), } \\
\text { repositories, governmental/ statistical offices, } \\
\text { databases, commercial providers, personal } \\
\text { connections, publications }\end{array}$ & $\begin{array}{l}\text { Following publication references; } \\
\text { survey banks - short queries, mismatch between } \\
\text { strategies and database design, DBK - more time } \\
\text { spent than in literature searching, keyword } \\
\text { searching followed by browsing, filters and author } \\
\text { names not used }\end{array}$ \\
\hline
\end{tabular}

Table 3.2: Actions taken to locate data

\section{Astronomy}

Astronomers are generally efficient information seekers, in part due to strong disciplinary infrastructures and tools (Meyer et al., 2011). SDSS users download data directly from NASA archives or obtain them from public data releases (Sands et al., 2012). Discovering and tracking down smaller datasets is challenging; SDSS users sometimes browse personal websites or use general search engines. They then contact research groups directly with their data requests. Despite well-developed infrastructures, personal networks remain an important means for identifying and obtaining data (Sands et al., 2012).

Journal articles are another important data source. Astronomers copy and paste or transcribe data from articles into new tables for further manipulation (Pepe et al., 2014). Direct citation of archival accession numbers facilitates data discovery from journals (Swan \& Brown, 2008).

\section{Earth and environmental sciences}

Finding and accessing biodiversity data can be challenging, although academics have an easier time than government employees and program managers. A lack of training, time, and knowing where to look hinders effective data search among these groups (Davis et al., 2014). Knowing where to search can be especially problematic in areas outside of a researcher's primary expertise (Devarakonda et al., 2011) and is contingent on knowing that 
data even exist (Zimmerman, 2003). Personal experiences with data collection and a familiarity with research trends help researchers estimate whether data are extant and findable (Zimmerman, 2007).

Compounding this problem, data are distributed across numerous repositories (Dow et al., 2015). Users must first discover the repository, and then invest significant time and energy becoming familiar with each search environment (Ames et al., 2012; Beran et al., 2009). Given the diversity of interfaces, it is not surprising that water scientists desire a "Google for data" (Megler \& Maier, 2012).

In a global survey of the environmental research community, the majority of respondents discover data through journal articles, search engines, and disciplinary repositories; $40 \%$ request data directly from data providers (Schmidt et al., 2016). Although some environmental planners are interested in using journals and primary sources, they find it too time-consuming (Miller et al., 2009), and may instead turn to colleagues for biodiversity information (Janse, 2006; Pullin et al., 2004).

Stratigraphers extract data from journals, laboriously re-creating tables from published graphs. They are willing to spend money as well as time obtaining data, sometimes purchasing expensive high-resolution data from drilling companies (Weber et al., 2012). Geographers utilize journals and search engines to locate maps, images, and repositories, but poor indexing and metadata derail their efforts (Borgman et al., 2005). Ecologists in Zimmerman's studies gather single data points from multiple sources and then aggregate them to create new datasets $(2007 ; 2008)$, an approach that is increasingly common in biodiversity research (Davis et al., 2014).

Personal exchanges are valuable, if complex, sources of external data. Requesting data from CENS, for example, is a multistep process. Data seekers identify CENS as a potential source, contact the CENS researcher, and discuss the availability and suitability of the data. The CENS researcher then gathers, processes, and delivers the requested data (Wallis et al., 2013). Ecologists employ a variety of tactics (emails, letters, and telephone calls) to obtain data mentioned in articles. As organizations grow and such requests increase, personal exchanges cease to be an effective way to obtain data (Wallis et al., 2007).

Ecologists reusing data employ "bounding" strategies, limiting searches to particular journals, times or locations to collect representative samples (Zimmerman, 2007). As data seeking is data collection, these researchers use strategies that minimize error, can be 
publicly defended, and increase the likelihood of accessing data (Zimmerman, 2007). They have specific search criteria; the general information in databases usually does not meet their detailed needs (Zimmerman, 2007). Before building specific search tools, CMOP researchers struggled with similar problems, retrieving either zero or thousands of hits. If researchers found searching too frustrating, they would simply stop searching (Maier et al., 2012; Megler \& Maier, 2012).

Large atmospheric datasets, encoded in binary formats to facilitate storage and transfer, cannot effectively be searched with text-based search engines. Rather, users must browse collections using metadata schemas (Pallickara, Pallickara, Zupanski, \& Sullivan, 2010). For other data, i.e. datasets in the DataONE platform, users prefer keyword searches, followed by filtering (Murillo, 2015).

\section{Biomedicine}

While it has become easier to locate data, for example in neuroscience (Beaulieu, 2004), access restrictions still frustrate researchers (Honor et al., 2016)

Medical image retrieval studies show that users search both local restricted-access systems and free internet sources. Local systems, including Picture Archiving and Communication Systems (PACS), electronic patient records, hospital archives, and teaching files, house images and patient data (Müller et al., 2006). Radiologists also curate their own collections of images stored on personal computers (Markonis et al., 2012).

Despite access to specialized collections, internet searches, particularly with Google Images, are common (Markonis et al., 2012; Müller et al., 2006). Limitations of such searches include sifting through irrelevant results and a dearth of highly-specialized images. Nevertheless, online image repositories are unpopular among health care professionals, perhaps because of their limited scope (Sedghi et al., 2011). Academic journals, however, facilitate locating specialized, cutting-edge images with contextual information that are difficult to locate on the web (Sedghi et al., 2011).

Search strategies vary depending on the searcher's professional role, although commonalities do exist. Users often search by patient names or identifier in PACS for diagnostic purposes; brief keyword or hierarchical searching is typical in non-diagnostic searching (De-Arteaga et al., 2015; Markonis et al., 2012; Müller et al., 2006). 
Success is not assured when searching for images. In a study of radiologists, users fail to find desired images in almost $25 \%$ of cases. Users believe these images exist, but that they simply cannot be found (Markonis et al., 2012). Possible search difficulties stem from a lack of time and available relevant papers, the newness of certain topics, and a lack of domainspecific search tools (Sedghi et al., 2011).

\section{Field archaeology}

Data discovery is a significant problem in field archaeology. Data are scattered among collections or sometimes are only in unpublished field reports (Niccolucci \& Richards, 2013). Although publications are used in data discovery (Faniel \& Yakel, 2017), they do not consistently include data; a significant delay between data collection and publication exacerbates the problem (Kriesberg et al., 2013). Researchers often do not know what data are available (Aloia et al., 2017). ECRs circumnavigate difficulties by collaborating with supervisors to locate data (Kriesberg et al., 2013). Other archaeologists turn to personal networks, museums, and, as the shift toward digital data continues, data archives (Faniel, Kansa, et al., 2013; Faniel \& Yakel, 2017). Details about how users search archives are sparse (Borgman, van de Sompel, et al., 2015), although searching and browsing by location are important strategies often complicated by differences in geographic terminology (Borgman et al., 2018).

\section{Social sciences}

Social scientists use data from governmental/statistical offices and specialized databases (Shen, 2007). Economists also obtain data from statistical offices but may purchase data directly from commercial providers (Bahls \& Tochtermann, 2013). Researchers easily locate data from national, publicly funded datasets, but struggle to locate smaller datasets and video data for reuse (Key Perspectives, 2010). Researchers tap publications or make direct requests to find this more specialized data (Swan \& Brown, 2008).

Personal networks, including advisors, co-workers of advisors or former employers, are key sources of qualitative data (Yoon, 2014b), especially for ECRs, who rely on journal recommendations from advisors and observations of their colleagues (Kriesberg et al., 2013; Faniel \& Yakel, 2017). Not knowing who to contact or where to begin searching makes locating relevant data difficult (Curty, 2016).

Searchers of the DBK, the primary catalog for social science data in Germany, expend more time and effort when seeking datasets than they do for publications. These researchers do not frequently use author names; rather, keyword searching, followed by browsing long 
results lists, are more frequent strategies. Researchers complain about a lack of filtering options, but do not use available filters (Kern \& Mathiak, 2015). Social scientists search a survey bank by short keyword queries or social construct, even though these strategies do not match the database's structure (Dulisch et al., 2015).

\subsubsection{Evaluation}

We identify major frames used in the literature to discuss data evaluation criteria, including trust, quality, necessary contextual information, and relevance. The frames overlap, as the characteristics composing these frames vary from paper to paper, both within and across disciplines. In the table below, we present the evaluation criteria and associated frames as they are discussed in the literature.

\begin{tabular}{|c|c|}
\hline $\begin{array}{l}\text { Users in this } \\
\text { community... }\end{array}$ & Use these criteria to evaluate data \\
\hline Astronomy & $\begin{array}{l}\text { 1. Contextual Information: instrumentation, observational conditions, data processing, original } \\
\text { research questions } \\
\text { 2. Trust: author reputation, source reputation }\end{array}$ \\
\hline $\begin{array}{l}\text { Earth \& Environmental } \\
\text { Sciences }\end{array}$ & $\begin{array}{l}\text { 1. Contextual Information: instrumentation, observational conditions, data collection procedures, } \\
\text { data processing, provenance, original research questions } \\
\text { 2. Quality: meet community standards, comprehensiveness/continuity over time, estimations and } \\
\text { uncertainties, resolution } \\
\text { 3. Trust: source, knowledge of object and data collector, author reputation/affiliation, funder, } \\
\text { community membership } \\
\text { 4. Understandability: familiarity with practices, data type, subject; consult experienced } \\
\text { researchers, first decode data } \\
\text { 5. Ease of access }\end{array}$ \\
\hline Biomedicine & $\begin{array}{l}\text { 1. Quality: noise, resolution, anatomical coverage, image acquisition details } \\
\text { 2. Trust: supporting documentation, social networks } \\
\text { 3. Relevance: experience, combination of textual/visual/medical criteria, visual relevancy, } \\
\text { background information, understandability, image quality, modality, source }\end{array}$ \\
\hline Field Archaeology & $\begin{array}{l}\text { 1. Contextual information: collection methods, instrumentation, observational conditions, } \\
\text { provenance, original research goals, baseline geographic/stratigraphic/chronological data } \\
\text { 2. Suitability for analysis: consistent data recording practices } \\
\text { 3. Trust: reputation/affiliation/skill of authors, repository features, language in supporting } \\
\text { documentation }\end{array}$ \\
\hline Social Sciences & $\begin{array}{l}\text { 1. Contextual Information: collection methods, instrumentation, other analyses, } \\
\text { definition/measurement of variables, data handling/processing } \\
\text { 2. Quality: completeness, accessibility, ease of use, credibility, reputation of repository, reputation } \\
\text { of author/journal not important } \\
\text { 3. Relevance: time frame of study, keywords, citing literature, title and publication year not as } \\
\text { important } \\
\text { 4. Trust: prior reuse, reputation of data repository, reputation of data producer }\end{array}$ \\
\hline
\end{tabular}

Table 3.3: Evaluation criteria with frames used in the literature 


\section{Astronomy}

Astronomers rely on detailed documentation of instrumentation, collection methods and conditions, data processing, and original research questions (Borgman et al., 2016; Wynholds et al., 2011). They know which authors to trust and believe data in NASA archives and established projects are valid, accurate, and trustworthy. Researchers must completely understand data and the creation processes; they would rather recreate data before using poorly documented secondary data products (Wynholds et al., 2011).

\section{Earth and environmental sciences}

When evaluating data for reuse, researchers use contextual information about data provenance (Dow et al., 2015; Murillo, 2015), technical instrumentation (Wallis et al., 2007), and original research questions (Zimmerman, 2008). Researchers reuse data they understand, seeking data collected via practices they have used themselves (Zimmerman, 2007; Zimmerman, 2008) and with familiar data types (Murillo, 2015). Contextual details are found in field notebooks (Weber et al., 2012) and articles (Carlson \& Stowel-Bracke, 2013), but additional metadata attached to datasets are the preferred method of conveying context (Bowker, 2000b). Formal metadata has limitations, though, as they cannot always contain enough detail or inspire the confidence needed for reuse. Researchers may instead base decisions on the word-of-mouth reputation of the dataset (Weber et al., 2012) or rely on more experienced researchers to develop understanding or alternative evaluation strategies (Zimmerman, 2008)

Data must have sufficient quality, often defined by community standards, to be reused (Zimmerman, 2007). Water researchers and earth science modelers consider comprehensiveness and continuity over time and space (Dow et al., 2015; Parsons, 2011) as well as uncertainties and error estimates (Larsen et al., 2016; Parsons, 2011) when determining data quality. Volcanologists use image resolution as a quality indicator (Weber et al., 2012).

Ecologists trust data from well-known sources, such as databases and literature (Zimmerman, 2007), and make decisions based on authors' reputations and affiliations (Murillo, 2015; Weber et al., 2012). Initial evaluations are based on the reputation of the source where the data were discovered, even if researchers eventually obtain them through other means (Zimmerman, 2007). Standardized collection practices are not enough to establish trust, as practices themselves say nothing about the data collector's skill (Zimmerman, 2008). The sponsor of research (McNie, 2007) and membership in the same 
community of practice (Van House et al., 1998) facilitate trust among environmental planners and policy makers.

Both ecologists and modelers reuse data that are easy to access (Zimmerman, 2007; Parsons, 2011). Modelers, however, face an extra step in the evaluation process, needing first to decode numerically encoded datasets before deciding if they are appropriate (Pallickara et al., 2010).

\section{Biomedicine}

Visual, medical, and textual criteria are used to evaluate biomedical images. Health care workers rank visual relevance, background information, and image quality as being most important, although they also mention image modality and understandability (Clough et al., 2008). Radiologists rely on a mixture of image properties, image quality, supporting documentation, and information about the source to determine suitability (Markonis et al., 2012).

Evaluation criteria vary depending on users' professional specialties and particular situations (Clough et al., 2008). Users rely on visual attributes when evaluating general medical images but incorporate textual information and credibility criteria for specific images used for background purposes (Sedghi et al., 2011).

Definitions of quality also vary by user. A neurosurgeon, for example, uses noise levels, resolution, and anatomical coverage, while a radiologist focuses mostly on motion artefacts to determine image quality (Heckel et al., 2016). Resolution and acquisition details (e.g. slice thickness in tomographic images) are other proxies for quality (Müller et al., 2006).

Healthcare professionals determine relevance through a combination of textual background information, visual inspection, and mental comparison to imagined ideals (Sedghi et al., 2012). Personal experience trumps other criteria, however, when determining image relevance (Markonis et al., 2012; Müller et al., 2006).

Clinicians build trust in images through supporting documentation, such as attached exams or biopsies. Systems allowing researchers to comment on images online can also build trust normally created through informal "hallway" communications (Jirotka et al., 2005; Markonis et al., 2012). 


\section{2 | Chapter 3}

\section{Field archaeology}

Archaeologists require contextual information about collection methods, instrumentation, observational conditions, and artefact provenance (Faniel, Barrera-Gomez, et al., 2013). Other fundamental metadata include information about original research goals and baseline geographic, stratigraphic, and chronological data (Atici et al., 2013). Current metadata schemas are not rich enough to provide this level of contextual description. Archaeologists either make do with the available information or seek other ways to further develop context (Faniel, Kansa, et al., 2013).

Consistent data recording practices (e.g. an absence of misspellings or translational errors) (Atici et al., 2013), and detailed language in supporting documentation (Faniel, Kansa, et al., 2013) help to establish credibility and trustworthiness. Author reputation and affiliation and repository features, such as metadata type and level of transparency, help to establish trust (Faniel, Kansa, et al., 2013)

\section{Social sciences}

DBK users spend more time evaluating data results compared to literature results, consulting additional documentation when needed. Researchers appear to think this is normal, perhaps because choosing the correct dataset is more important than selecting the correct article (Kern \& Mathiak, 2015). Title and publication year are not as important as study time frame and keywords in evaluations. Users would like access to literature citing a dataset to determine if a research question has already been answered (Kern \& Mathiak, 2015); prior reuse of data is also an important way of developing trust (Faniel \& Yakel, 2017).

Data seekers rank accessibility as the most important factor determining satisfaction with data reuse in the ICPSR repository. Data completeness (ranked $2^{\text {nd }}$ ), credibility $\left(4^{\text {th }}\right)$ and ease of use $\left(5^{\text {th }}\right)$ are also contributing factors; in this study, journal/author reputation do not appear to impact satisfaction (Faniel et al., 2016). Other work suggests that the repository reputation is an important signal of data quality and credibility (Curty, 2016) and is used to develop trust in data (Faniel \& Yakel, 2017). Data re-users tend to either make do with available data or adapt their research projects to use data that they can find. The more researchers have to "reshape" their projects, the less satisfied they are (Faniel et al., 2016).

Users need contextual information about collection methods, instrumentation, other analyses, and how variables are defined and measured (Curty, 2016; Faniel, Kansa, et al., 2013; Kern \& Mathiak, 2015; Yoon, 2014a). When necessary, researchers turn to other 
sources to develop the necessary context (Fielding \& Fielding, 2008), consulting colleagues, codebooks (Faniel \& Yakel, 2017) or bibliographies (Faniel, Barrera-Gomez, et al., 2013). Ideally, specialized metadata schemas would provide enhanced context (Kern \& Mathiak, 2015). Debate remains, however, if documentation can build the context needed to reuse qualitative social science data (Broom et al., 2009; Parry \& Mauthner, 2005).

Novice researchers especially need supporting contextual information. They want details about coding procedures, collection methods and dataset merging and matching (Faniel et al., 2012). More experienced researchers can make do more easily with limited documentation (Yoon, 2016).

\subsection{Discussion}

Having presented the documented practices of observational data users, we use the frameworks to synthesize our key findings and to identify commonalities and themes spanning the reviewed disciplinary communities.

\subsubsection{Users and needs}

Researchers across and within the reviewed disciplines need a diversity of observational data, requiring data of different types from different sources and disciplines, collected at different scales using different instruments. Users have very specific requirements, needing data from particular locations (geographic, anatomical, or astronomical), at particular resolutions or collected using particular mechanical or survey instruments.

Integrating diverse data is necessary but challenging. Astronomers struggle to bring together data from different telescopes, neuroscientists try to combine neuroimages with clinical data, and archaeologists need to integrate data collected in different contexts with different methodologies. Some of these challenges may be augmented by changes in research practices, such as automated data collection in EES (Borgman et al., 2007), or by shifts in community data practices, such as increased data sharing, as in archaeology (Arbuckle et al., 2014) or neuroscience (Choudhurry et al., 2014).

Background and foreground uses are reported across disciplines, although background uses are better documented. These include making comparisons, benchmarking, preparing research projects, calibrating instruments, and as model inputs. Reported foreground uses are vaguer, often limited to reports of "asking new questions of data." This does not mean that foreground uses do not occur; examples of new research fueled by data reuse could 
likely be found in all of the reviewed disciplines (e.g. Atici et al., 2017). This could indicate a mismatch between what studies of data practices report and actual practices, or it could be a sign of changing practices. Even with a broad analysis, we see that data use varies within disciplines. One group of biodiversity researchers uses secondary data only to support projects, for example, while another study only examines cases of foreground use. Other possible data uses, i.e. in teaching, clinical practice, or environmental planning, are hinted at, although rarely explored in detail.

A generic view of the user is also common. Similar to our approach, disciplines are often broadly represented; the social sciences in particular tend to be treated as a homogenous group. Few studies document the needs and behaviors of specific user groups, such as early career researchers (Kriesberg et. al, 2013; Faniel et al. 2012), policy makers (Janse, 2006; McNie, 2007; Cash et. al, 2003) or students (Carlson \& Stowel-Bracke, 2013). Understanding the data practices of ECRs sheds light on processes of acculturation (Kriesberg et al., 2013) and is important, as large-scale data reuse depends on adoption by ECRs (Faniel et al., 2012). Understanding the practices of specific user groups is also critical in designing user-oriented data discovery systems.

\subsubsection{User actions}

Across communities, users find data in repositories, journals, on websites, and through personal networks. This variety could be due to differing infrastructures available within disciplines; however, even in fields with established data repositories, i.e. astronomy and quantitative social science, researchers seek data outside of these systems (Sands et al., 2012; Faniel \& Yakel, 2017).

Personal exchanges are valuable sources of external data. While locating large, well-known datasets is straightforward, tracking down smaller, specialized datasets is challenging and often requires personal communication (Sands et al., 2012). Existing repository search functionalities may not meet the specific needs of researchers, or users may not develop appropriate search strategies in these resources (Sedghi et al., 2011). Users may also simply not be aware of the existence of data or databases; this may be especially true for researchers seeking data outside of their primary disciplines.

The distributed nature of observational data compounds these problems. A variety of data repositories exist within these disciplines (e.g. Dow et. al, 2015); within each new resource, users must start from scratch - first discovering the resource, then investing significant time 
and energy becoming familiar with it and the available data. A lack of time and accessible data also complicates the search process.

\subsubsection{Evaluation}

Researchers across disciplines need as much contextual information as possible, requiring documentation about instruments, methodologies, research questions, and observational conditions. This information is combined with the reputation of the repository and often that of the data author to establish trust, data quality, and relevance. Although much of the reviewed literature uses frames such as trust and quality to discuss evaluation, the characteristics used to develop these frames varies. This variation may result from disciplinary or individual differences or from how the papers' authors define these frames. One commonality that we can identify is the association of more social criteria - such as the reputation of authors and data sources - in developing trust.

Enriched metadata are often the desired, although imperfect, method of conveying contextual information. Perhaps because of limitations in metadata, researchers build the needed information by combining a variety of sources, from codebooks and academic literature to unpublished reports and museum records (Faniel \& Yakel, 2017). Researchers across communities also use social connections and personal exchanges to evaluate data. The discussion about how researchers evaluate data is still developing, although the process seems to differ from how researchers evaluate academic literature.

The following themes bridging both frameworks emerge from this synthesis:

- A tension between breadth and specificity

- The social aspects of data retrieval

- Absent practices and communities

When developing the frameworks for this paper, we presented the tension involved in applying broad perspectives to understand individual practices. This tension between breadth and specificity is also present in the reviewed data retrieval practices. Even within disciplines, researchers need a diversity of observational data and employ a wide variety of search and evaluation strategies. At the same time, users seek data with very precise characteristics. They appear to balance breadth and specificity as they work to integrate datasets from diverse sources to meet specific needs or to piece together a variety of evaluation criteria to make decisions about reuse. 
Social connections and personal exchanges permeate observational data retrieval. Users rely on personal connections and their own networks to locate, obtain, and evaluate data, even in disciplines with extensive infrastructures. This suggests that it is not enough to understand data retrieval as a series of interactions between users and search systems; rather, data retrieval is in fact a complex sociotechnical process.

The absence of many communities and practices in the literature is also apparent. A relatively small number of disciplines are represented in our literature corpus. Among the broad disciplinary categories that we employ, certain subdisciplines are well represented; others are briefly mentioned, and others are treated homogeneously. Building a robust picture of observational data retrieval requires a deeper understanding of practices in other disciplines and of understudied user groups such as non-scientists or early career researchers. Deeper studies of how data retrieval practices change when seeking data for foreground purposes, or when seeking data from different disciplines, are also absent. Although Faniel \& Yakel have recently identified five "trust markers" important in data reuse in archeology, social sciences and zoology (2017), common frameworks for discussing evaluation criteria across the observational data community are lacking.

\subsection{Conclusion: Towards a model for data retrieval}

Through our analysis we have achieved the following:

- Shown that a framework based on interactive information retrieval is applicable to understanding the data retrieval literature

- Tested the boundaries of defining data communities, using broad classifications to identify commonalities in practices

- Revealed absent practices and highlighted areas where more research is necessary

- Suggested that a framework based on IR alone is insufficient for completely understanding the complexity of data retrieval practices.

The literature also points to ways that information retrieval and data retrieval differ. Data needs are specific, requiring high precision in IR systems (Stempfhuber \& Zapilko, 2009). Textual queries and ranking algorithms do not work well for retrieving numeric or encoded data (Pallickara et al., 2010). Users employ different search strategies when seeking data than literature (Kern \& Mathiak, 2015) and take different roles when interacting with data repositories (e.g. as consumers and creators), which can impact system design (Borgman, 
van de Sompel, et al., 2015). Researchers also spend more time evaluating datasets (Kern \& Mathiak, 2015), perhaps because lists of data cannot be efficiently evaluated in the same way as document lists (Kunze \& Auer, 2013).

These differences, in conjunction with the themes identified in the discussion, suggest that current information retrieval models may not completely describe data retrieval practices. Identifying commonalities in observational data retrieval practices is a first step in exploring possible characteristics of a new model for data information retrieval. Further studies of different data communities, such as users of experimental and computational data, big and long-tail data seekers, and members of underrepresented user groups are needed. A model describing data retrieval would provide insight into the needs and practices of users that could be applied to both systems design and policy developments for facilitating data discovery.

\section{Acknowledgements}

KG developed the frameworks, collected the data, and wrote the manuscript. PG, HC, SW, and AS contributed to theory development and editing. This work was funded by the Dutch Research Council (NWO), Grant 652.001.002. 
CHAPTER 4 


\section{Understanding data search as a socio-technical practice}

Originally published as: Gregory, K. M., Cousijn, H., Groth, P., Scharnhorst, A., \& Wyatt, S. (2019). Understanding data search as a socio-technical practice. Journal of Information Science. https://doi.org/10.1177/0165551519837182 


\section{0 | Chapter 4}
Abstract
Open research data are heralded as having the potential to increase effectiveness, productivity, and reproducibility in science, but little is known about the actual practices involved in data search. The socio-technical problem of locating data for reuse is often reduced to the technological dimension of designing data search systems. We combine a bibliometric study of the current academic discourse around data search with interviews with data seekers. In this article, we explore how adopting a contextual, socio-technical perspective can help to understand user practices and behavior and ultimately help to improve the design of data discovery systems. 


\subsection{Introduction}

The reuse of open research data is heralded as having the potential to increase effectiveness, productivity, and reproducibility in science (Gray, 2009; Wilkinson et al., 2016). However, data do not flow easily between users, situations, and disciplines (Borgman, 2015a). Instead, they rely on dynamic relationships between people, context and technology.

We aim to explore these relationships in an ongoing project that integrates science and technology studies and information science to inform and intervene into the design of tools for searching for research data. In this paper, we answer recent calls to integrate scientometric studies and qualitative methods (Wyatt et al., 2017) by combining bibliometrics and interviews to gain empirical evidence about researchers' data search practices.

Interest in facilitating data sharing and reuse is high. Funding agencies, research organizations, and repositories are all increasingly engaged in drafting policies regulating data sharing and management (Digital Curation Centre, n.d.). Many studies mirror recent policy developments, focusing on data sharing and management practices, usually presenting the researcher in the role of data creator (Tenopir et al., 2011; Kennan \& Markauskaite, 2015). As more work is done to investigate how data are used, researchers' multiple roles are being recognized. Researchers are not only data producers, but they also act as consumers, curators, and evaluators of data (Borgman, Van de Sompel, et al., 2015).

Before data can be reused, they must first be discovered. Researchers seek, access, and evaluate data they have not created themselves as they engage in the process of searching for data. Data search has recently emerged as a separate topic of inquiry within the core information retrieval community (Groth et al., 2018). Here, research has focused primarily on finding technical solutions (Gregory, Groth, et al., 2019), with the development of ontologies, standards, and search tools taking precedence (Ohno-Machado et al., 2017; Sansone et al., 2017). Investigations into the social aspects of data search, such as data discovery and reuse practices, are far less common (Borgman, Darch, et al., 2016; Borgman, Wallis, \& Enyedy, 2007.

Our aim is to build on these investigations to understand data search from a socio-technical perspective. How do data (re)users locate and make sense of research data, and for which purposes? How are these practices situated with regard to technical resources and within communities? 
We tackle these questions using two methods. We begin by reviewing the data search literature with a bibliometric analysis, further revealing the technical bias, the distributed nature of the discourse, and gaps in terms of data search processes. We then present the results of interviews across disciplinary domains. The interview questions, informed by both our bibliometric study and dialogue with the designers of a data search system, explore data users' needs and contexts, their strategies for locating data, and the criteria brought to bear when evaluating reuse potential. Inspired by the quantitative analysis, we present our qualitative interview data using a unique tabular presentation

In our analysis, we view context as a collection of interacting, dynamic components (Lievrouw, 2001), where users are often simultaneously embedded in multiple contexts with varying social norms (Burnett et al., 2014). We also draw on the conceptualization of users as "social actors" (Lamb \& Kling, 2003), who internalize and act on the social and information norms of their communities (as summarized by Courtright, 2007). We conclude by discussing how data search can be understood as a socio-technical process rooted in context and pose suggestions for integrating these insights into the design of data discovery systems.

\subsection{Bibliometric study and analysis - Method I}

Research on data search currently focuses on technical challenges and solutions for searching data, as techniques for document-based retrieval do not work well for structured data (Cafarella et al., 2011). In response, researchers seek ways to apply keyword searching to datasets (Lopez-Veyna et al., 2012), to semantically enhance datasets (Khan et al., 2016), and to create new ontologies and standards (Ohno-Machado et al., 2017; Sansone et al., 2017). These approaches are used to create a variety of search tools for specific disciplines (Park et al., 2017; Megler \& Maier, 2013) and data types (Markonis et al., 2017; Dulish et al., 2015).

Studies of users' practices are not as prevalent. A small body of work examines how users seek and evaluate data within disciplines (Davis et al., 2014; Zimmerman, 2007; Zimmerman, 2008), across data-related professions (Koesten et al., 2017) or within data repositories (Kern \& Mathiak, 2015; Borgman, Van de Sompel, et al., 2015; Borgman, et al., 2018; Murillo, 2015). Much information on data search practices is buried within investigations of other data behaviors, such as studies investigating the characteristics of data sharing and (re)use in specific research teams and disciplines (Wynholds et al., 2012; Wallis, et al., 2013; Curty et al., 2016; Faniel, Kansa, et al., 2013). Work investigating the 
qualities of successful data reuse (Yoon, 2016) and that examine criteria that researchers use to establish data trustworthiness, e.g. the identity of the data creator, the reputation of a repository, or prior usage, (Faniel \& Yakel, 2017) is especially relevant when examining how users evaluate and make sense of data. Trust development in particular is recognized as a complex, non-linear, social enterprise (Yoon, 2017a).

A broader bibliometric analysis of the data search discourse makes this imbalance between technical and social research explicit. Building upon our earlier review of observational data users (Gregory, Groth, et al., 2019), we searched the literature using different keyword combinations across all fields, primarily in Scopus. We combined keywords related to information retrieval (e.g. user behavior, information seeking, information retrieval), data practices (e.g. research practices, community practices) and research data (see Figure 4.1). We performed other keyword searches for data search and discoverability and applied bibliometric techniques such as citation chaining and related records. Pertinent sources (journals, book series, proceedings, etc.) not indexed by Scopus were searched directly using similar keywords. We closely read the 400 retrieved documents to identify relevant publications, resulting in a final corpus of 189 documents published between 1990 and 2017 .

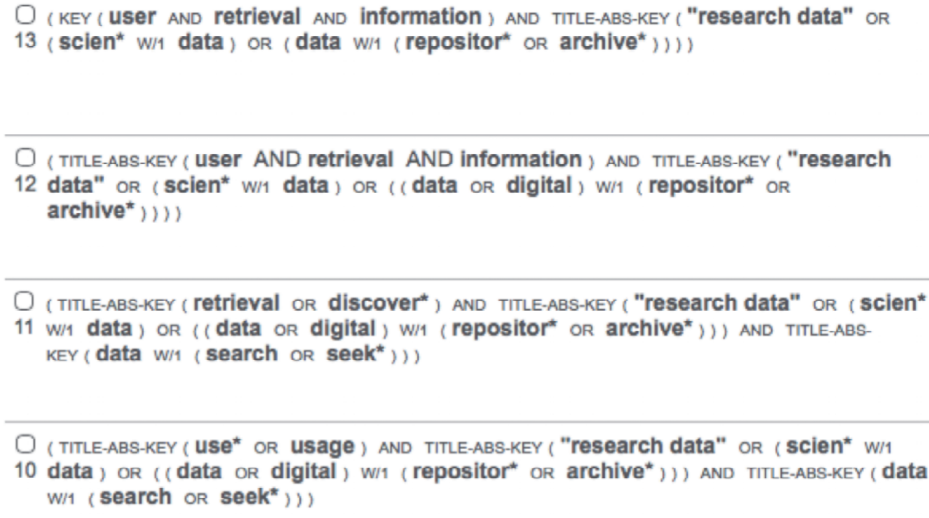

Figure 4.1. A sampling of keyword combinations used in Scopus

Restricting our results to journals and conference proceedings produced a corpus containing 102 sources titles and 182 publications. As Bradford's law predicts (Bradford, 1950), a small, core group of sources accounts for the majority of publications; only ten sources have published more than three publications. Most publications in these ten sources were published after 2012, indicating that interest in the topic has only recently begun to solidify (Figure 4.2). Scopus classifies seven of these top sources as computer science or 
engineering titles, highlighting the technical dominance in the discourse. We also see some hint of discipline-specific interest, evidenced by the appearance of Nucleic Acids Research and Bioinformatics.

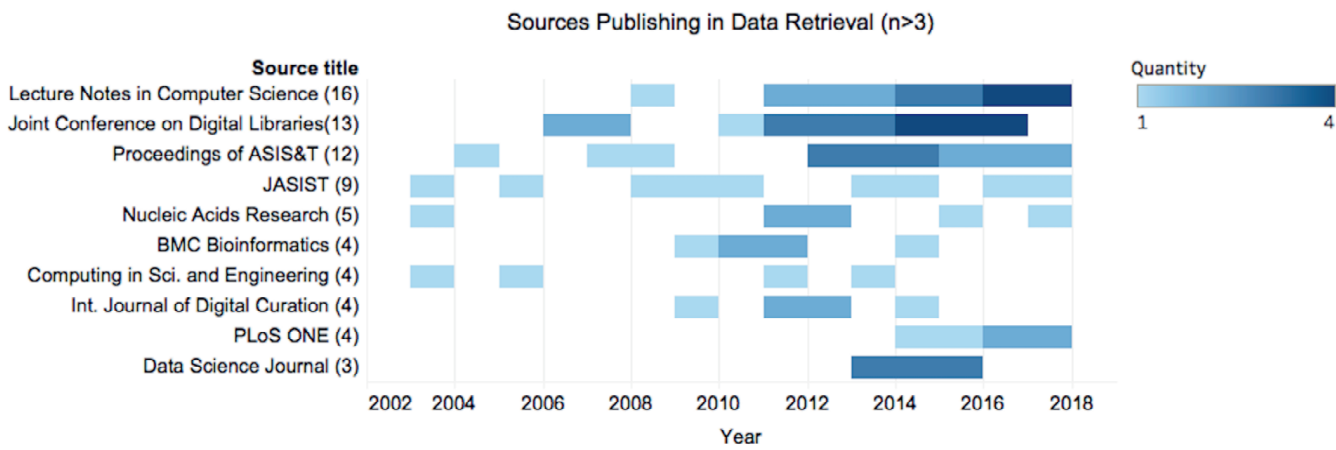

Figure 4.2. Sources in the corpus with more than three publications

The publications in Figure 4.2 include approximately $40 \%$ of the corpus. The remaining $60 \%$ are spread across other sources, including disciplinary journals which only appear once. The two earliest publications come from molecular biology and astronomy, indicating an early interest within traditionally data-intensive disciplines.

To augment the source-level analysis, we created a keyword co-occurrence map for all keywords using VOSviewer (Van Eck \& Waltman, 2010; Figure 4.3). The minimum cooccurrence was set to five; general keywords such as "article" or "procedures" were excluded. 


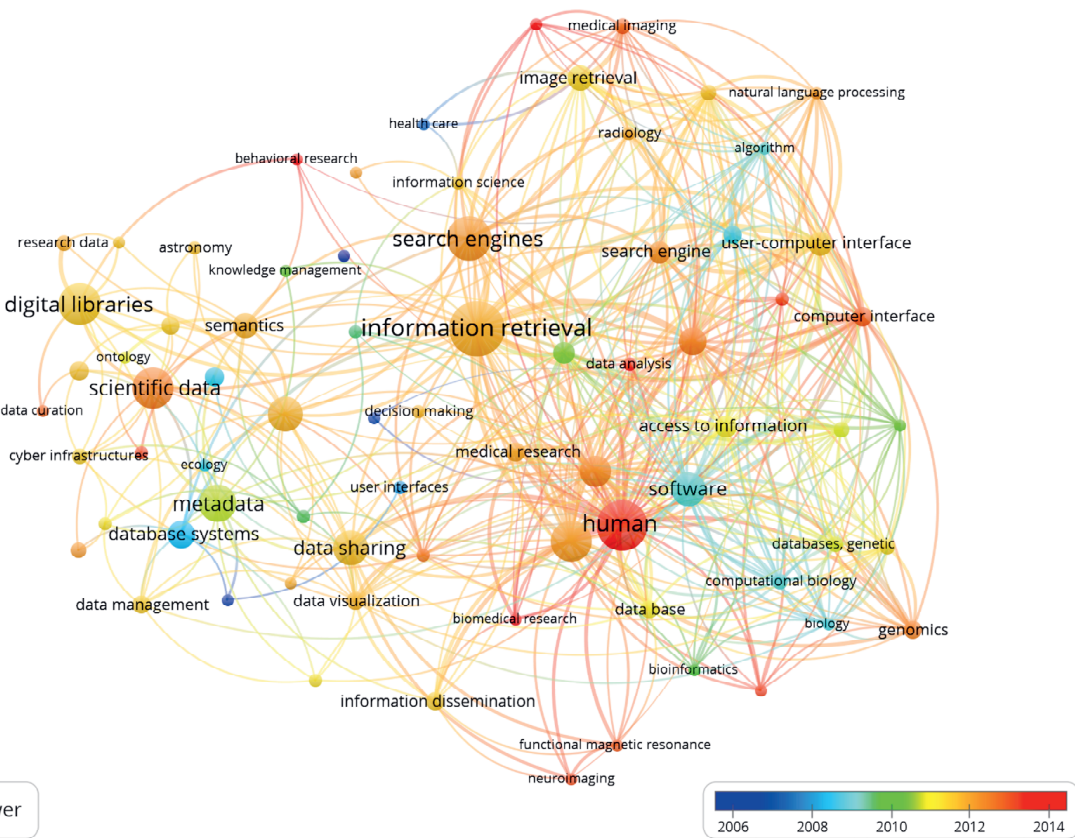

Figure 4.3. Keyword co-occurrence map with temporal overlay

Figure 4.3 demonstrates the spread of the discourse and its technical focus. We see the dominance of disciplinary keywords (e.g. genomics, astronomy) and the prominence of more technical areas (information retrieval, search engines, and image retrieval). The concept of human solidifies only recently; the highest average number of keywords appears in 2014, both in connection to technical concepts (information retrieval, search engines) and keywords associated with the study of humans (neuroscience, genomics).

The keyword mapping highlights gaps in the field. Only a handful of disciplinary keywords are present, mirroring the disciplinary bias in the source analysis. Fields with well-developed data infrastructures, such as high-energy physics (Dallmeier-Tiessen \& Mele, 2014), are surprisingly under-represented as are the social sciences. "Data search" or "data retrieval" are not identified as stand-alone topics, suggesting that information about these practices is buried within other discussions and that these terms are not yet codified labels. The analysis also suggests that social factors have only recently begun to be addressed.

Kacprzak and colleagues echo this idea, noting that existing data search applications are often based on preliminary or anecdotal evidence (Kacprzak et al., 2017). Query log 
analyses can help to develop insights about data search practices (Kacprzak et al., 2017), but log data cannot explain the reasoning behind search behaviors (Borgman et al., 1996). Our work begins from a deeper engagement with users themselves, conducting interviews informed by the findings of this bibliometric analysis and conversation with creators of a data search system. We thereby attempt to facilitate a dialogue between system designers and end users, bridging the social and the technical aspects of the problem while using scientometrics to inform qualitative research.

\subsection{Interview design and analysis - Method II}

\subsubsection{Development of interview protocol}

We drafted our interview questions to draw out the data needs, search practices, and evaluation behaviors of participants in a variety of disciplines. The development of our interview protocol was informed by the bibliometric study, our previous survey of the data search literature (Gregory, Groth, et al., 2019) and consultations with the Elsevier Data Search ${ }^{5}$ design and implementation team. Data Search is a publicly available data search engine that harvests data from multiple data and publication repositories (Scerri et al., 2016). It is currently under development; its design is not yet finalized. In the development process, the design team has conducted preliminary evaluations of user needs and behaviors. We used these evaluations as input to our interview protocol.

\subsubsection{Interviews}

We conducted 22 one-hour, semi-structured interviews between October and December 2017, using Skype, GoToMeeting or in-person meetings. Participants were recruited via email from a pool of 186 individuals who had visited the Data Search portal and had indicated willingness to provide feedback. We postulated that these individuals would have interest in searching for research data. We spoke with 19 respondents from the Data Search pool and recruited three additional participants using convenience sampling. We obtained ethical review for the study, and all participants provided written informed consent.

The majority of participants are active researchers, although some are active in other areas or have numerous roles (Table 4.1); participants also spoke about previous experiences in other roles or the experiences of their colleagues. Participants work in twelve countries. The

\footnotetext{
${ }^{5}$ datasearch.elsevier.com
} 
most frequently represented countries are the United States $(n=6)$ and the Netherlands $(n=3)$. Some participants currently work outside of their home countries or have past experience working abroad.

Participants self-identified their broad disciplinary area. Although a range of disciplines are present, computer science $(n=3)$ and information science $(n=3)$ are the most common. Individuals working in support roles, especially in libraries, have insight into the needs and practices of various disciplines $(n=3)$. Our participants are in diverse career stages: early career $(0-5$ years, $n=5)$, mid-career $(6-15$ years, $n=10)$, experienced $(16+$ years, $n=6)$, and retired $(n=1)$.

\begin{tabular}{|c|c|c|c|c|c|}
\hline $\begin{array}{l}\text { Partici- } \\
\text { pant }\end{array}$ & Gender & Discipline & $\begin{array}{l}\text { Country of } \\
\text { employment }\end{array}$ & Career Stage & Current role \\
\hline 1 & $\mathrm{M}$ & Medical statistics & USA & Retired & Citizen \\
\hline 2 & $M$ & Business and & UK & Experienced & Industry \\
\hline 3 & $\mathrm{~F}$ & $\begin{array}{l}\text { Cognitive } \\
\text { psychology, }\end{array}$ & Netherlands & Early & Researcher \\
\hline 4 & $\mathrm{~F}$ & Evolutionary & France & Early & Researcher \\
\hline 5 & $M$ & Information & Singapore & Early & Researcher \\
\hline 6 & $\mathrm{~F}$ & Water resources & Malaysia & Early & $\begin{array}{l}\text { Researcher, } \mathrm{PhD} \\
\text { candidate }\end{array}$ \\
\hline 7 & $\mathrm{~F}$ & Computer science & Australia & Middle & Researcher \\
\hline 8 & M & Computer science & USA & Middle & Researcher \\
\hline 9 & $M$ & Information & Spain & Middle & Researcher \\
\hline 10 & M & $\begin{array}{l}\text { Information } \\
\text { science, }\end{array}$ & Netherlands & Middle & Researcher \\
\hline 11 & $M$ & Psychology & Spain & Middle & Researcher \\
\hline 12 & M & $\begin{array}{l}\text { Psychiatry, } \\
\text { medicine, } \\
\text { neuroscience }\end{array}$ & Portugal & Middle & $\begin{array}{l}\text { Researcher, } \\
\text { clinical practice, } \\
\text { PhD candidate }\end{array}$ \\
\hline 13 & $\mathrm{~F}$ & $\begin{array}{l}\text { Cellular/molecular } \\
\text { biology, medical }\end{array}$ & USA & Middle & $\begin{array}{l}\text { Researcher, } \\
\text { industry }\end{array}$ \\
\hline 14 & M & $\begin{array}{l}\text { Computer } \\
\text { science, data }\end{array}$ & Guatemala & Middle & $\begin{array}{l}\text { Researcher, } \\
\text { industry }\end{array}$ \\
\hline 15 & $M$ & Acoustical & Canada & Experienced & Researcher \\
\hline 16 & $M$ & Industrial ecology & Brazil & Experienced & Researcher \\
\hline 17 & $M$ & Palaeontology & Netherlands & Experienced & Researcher \\
\hline 18 & $\mathrm{~F}$ & Popular culture & USA & Experienced & Researcher \\
\hline 19 & M & Chemistry & Australia & Experienced & $\begin{array}{l}\text { Researcher, } \\
\text { industry }\end{array}$ \\
\hline 20 & $\mathrm{~F}$ & Libraries & USA & Early & Support \\
\hline 21 & $\mathrm{M}$ & Libraries & UK & Middle & Support \\
\hline 22 & $\mathrm{~F}$ & $\begin{array}{l}\text { Scientific literature } \\
\text { manager }\end{array}$ & USA & Middle & Support \\
\hline
\end{tabular}

Table 4.1. Participant description 
Although participants were recruited from Data Search, the interviews did not focus on this tool. Rather, our aim was to learn more about general data search practices. We therefore asked open-ended questions, encouraging rich discussions about contexts and data needs, strategies for locating data and criteria for evaluation. We focused on data not created by participants, but otherwise left the term "data" open for definition by participants.

When necessary, we followed our questions by prompts to elicit more detail, some reflecting the interests of the search engine team. We audio-recorded the interviews and created detailed summaries for each interview. These summaries were uploaded into the qualitative data analysis program QDA Miner Lite for coding and analysis.

\subsubsection{Interview findings}

Our main findings are presented along three dimensions, informed by the analytical framework we have discussed elsewhere (Gregory, Groth et al., 2019). These three dimensions - user contexts and data needs, search strategies, and evaluation criteria - are further divided into non-hierarchical subsections designed to orient the reader to the interpretations presented in the discussion. We summarize our interview findings in tabular form; we do this not to indicate statistical meaning, as our sample size is small. Rather, we use the tables to provide an overview that facilitates navigating the discursive analysis of our interview findings.

\section{User contexts and data needs}

An important contextual aspect is a user's disciplinary community. For some, data sharing and reuse are normal, as in computer science, biomedicine, and astronomy $(7,13,20)$. For others, seeking data involves calculated exchanges, where information is only shared with individuals who share reciprocally (2).

Within disciplines, ideas of data ownership, data sharing regulations, data types, and cultural differences also affect participants' practices.

We have to be very careful sharing the raw data, otherwise we can be sued by the lab or by the university...In terms of the clinical data (in Portugal), the situation is different... it is not that formal as in the United States. It is quite frequent to share clinical data with other researchers (12). 
A lack of standardization in collection, description and sharing practices, even within disciplines where data sharing is established, can negatively affect data discoverability, evaluation, and reuse (8).

The data user is not always the person searching for data. Experienced researchers delegate some search responsibilities to graduate students, training students to find data for background purposes primarily by searching the literature $(13,16)$. Librarians and those in support roles assist students (20), external researchers (21), and those working in industry (22) to locate data.

Not all data seekers are involved in research. One participant does not have an academic research background but is interested in finding data from medical studies in the press (1). Others work in industry; an increasing number of students are seeking data (20).

\section{Diverse and changing needs.}

Participants need a variety of data for a variety of purposes. Chemists need numerical data such as superconducting temperatures and non-numerical spectral data; an evolutionary ecologist requires physiological data and field observations; and a humanist seeks social media posts for textual analysis. Data are needed for background purposes supporting research (Table 4.2) and for foreground purposes (Table 4.3) driving new research. 


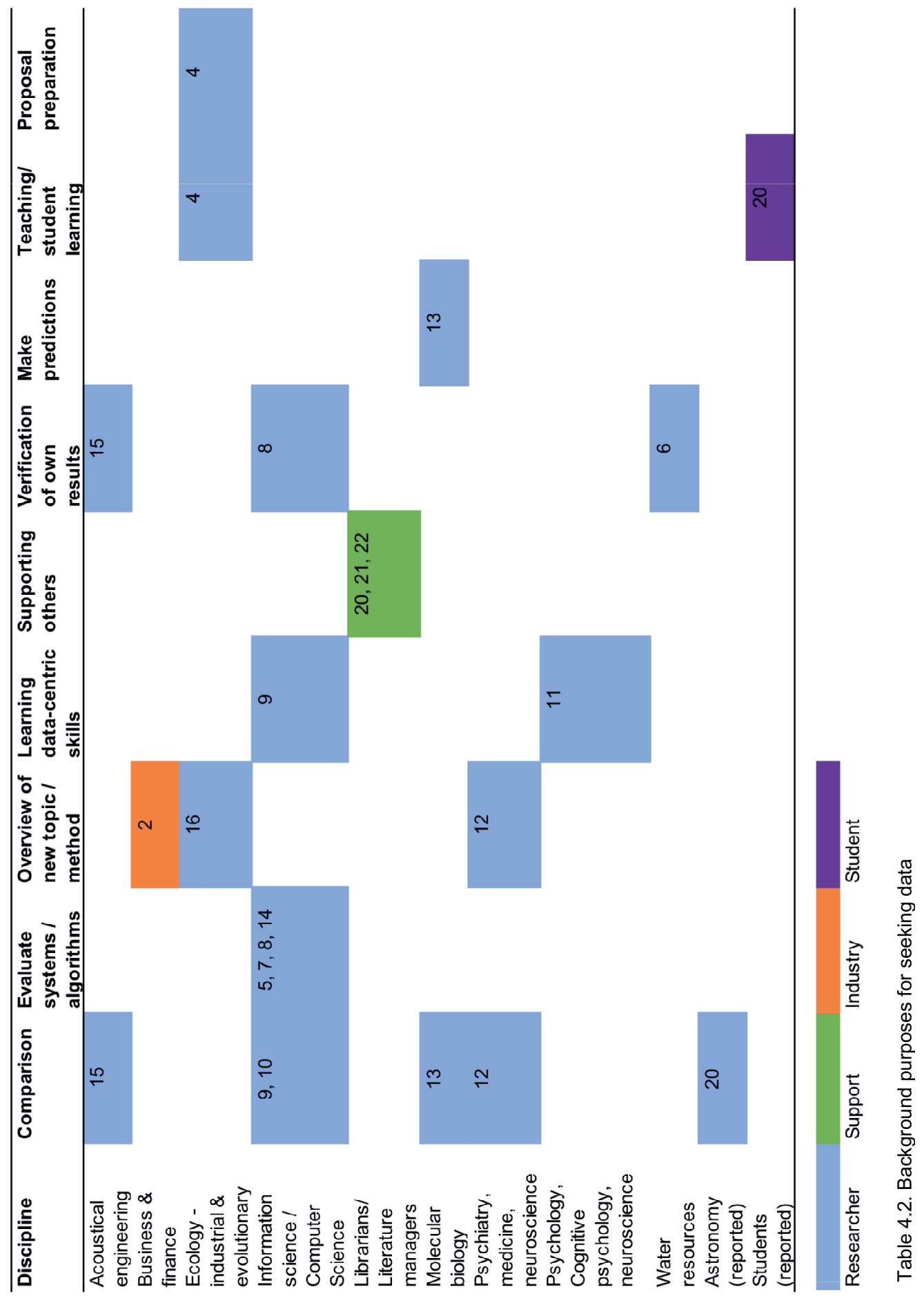




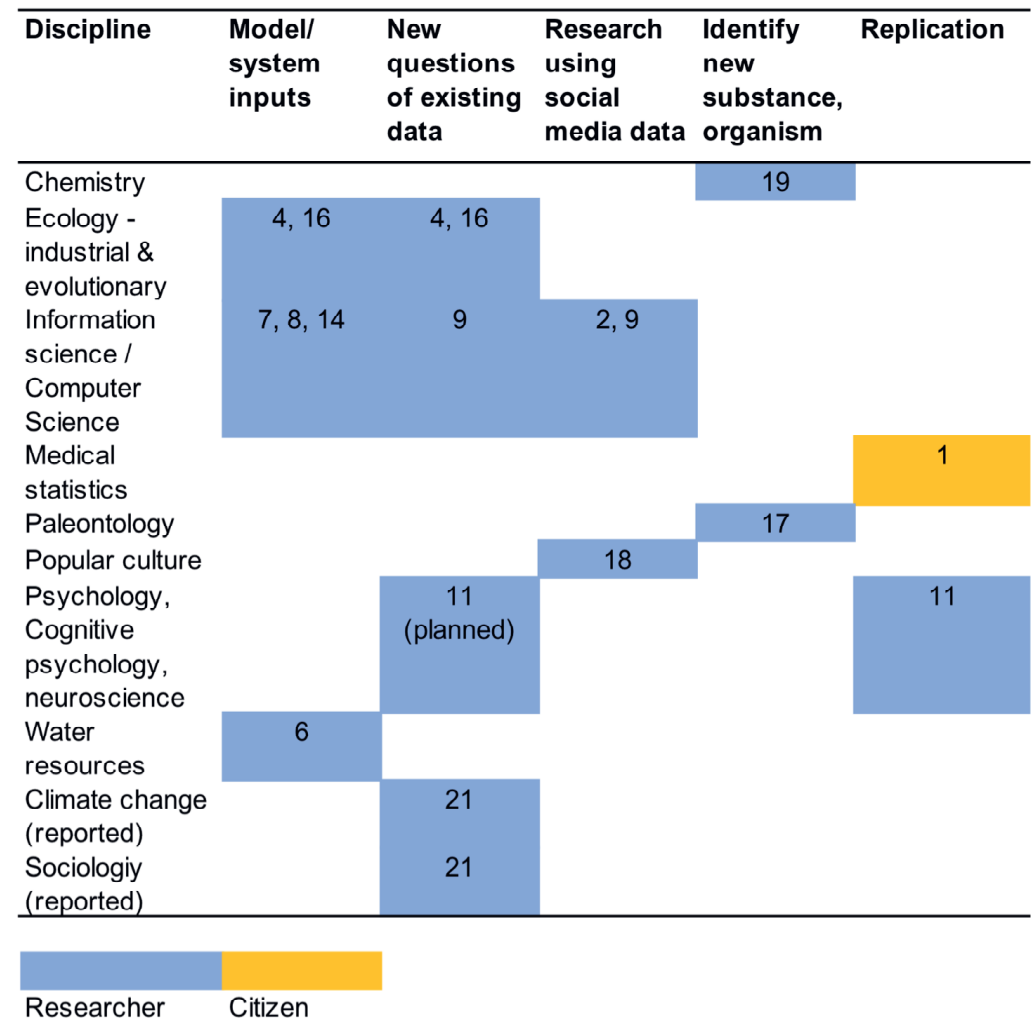

Table 4.3. Foreground purposes for seeking data

Participants need data from their own disciplines, but they also require other data. Across domains, participants use data created on social media platforms or data produced by governments. Researchers are also interested in data created for another purpose in other disciplines, e.g. sociologists seeking data collected by female freshwater biologists in the early $20^{\text {th }}$ century (21), or engineers building a biomimicry database (17).

Descriptions about data can be as important as the data themselves. Paleontologists rely on descriptions about physical specimens in the literature to trace evolution over time. Specimens are sometimes destroyed or inaccessible, bestowing extra value to these descriptions (17).

Metadata are crucial for computer and information scientists, who need rich metadata to design and test systems. 
I am not really using observation values. I am more using the description of the datasets, the metadata...Also I am using secondary metadata. The datasets are linked to publications, and I am using metadata about the publications (7).

At other times, computer and data scientists need large multidisciplinary datasets with minimal indexing to create indexing systems or to test algorithms (14).

Research interests determine the data that participants need. As interests change, so do data needs. Sometimes these changes result from new subject interests (11) or job changes (4). Needs can also evolve in response to the changing nature of data and the research environment. As more data become openly available, some researchers are curious about using open data or experimenting with data science approaches:

A new approach would be pulling large datasets into some data mining process. This is something that big data scientists do. Sometimes they find specific relationships between variables, that apparently don't have anything to do (with each other). They don't know how to interpret these relationships. But we psychologists and social scientists actually have the theory and the background to interpret these relationships. (11)

These projects demand datasets of increasing size and require researchers to develop new data analysis skills (9).

Participation in interdisciplinary projects is common. This is mirrored in an observed increase in interdisciplinary research institutes at the university level; these institutes provide an opportunity for researchers across disciplines to encounter and use each other's data (21).

\section{Search strategies}

Multiplicity of resources and strategies.

Most participants employ a mixture of strategies and visit multiple resources to find data (Tables 4.4 and 4.5). The majority use Google either to search for data or to locate repositories; the success of these searches is mixed.

I just search for keywords [in Google] - prostate, MRI, segmentation...but it is not very effective. I can't remember a single instance when I found something useful. What people don't realize is that in medical imaging, data has to be in a special format. If 
you just search for prostate images, you will see a lot of pretty pictures, JPEGS, but that is not suitable for the analysis (8).

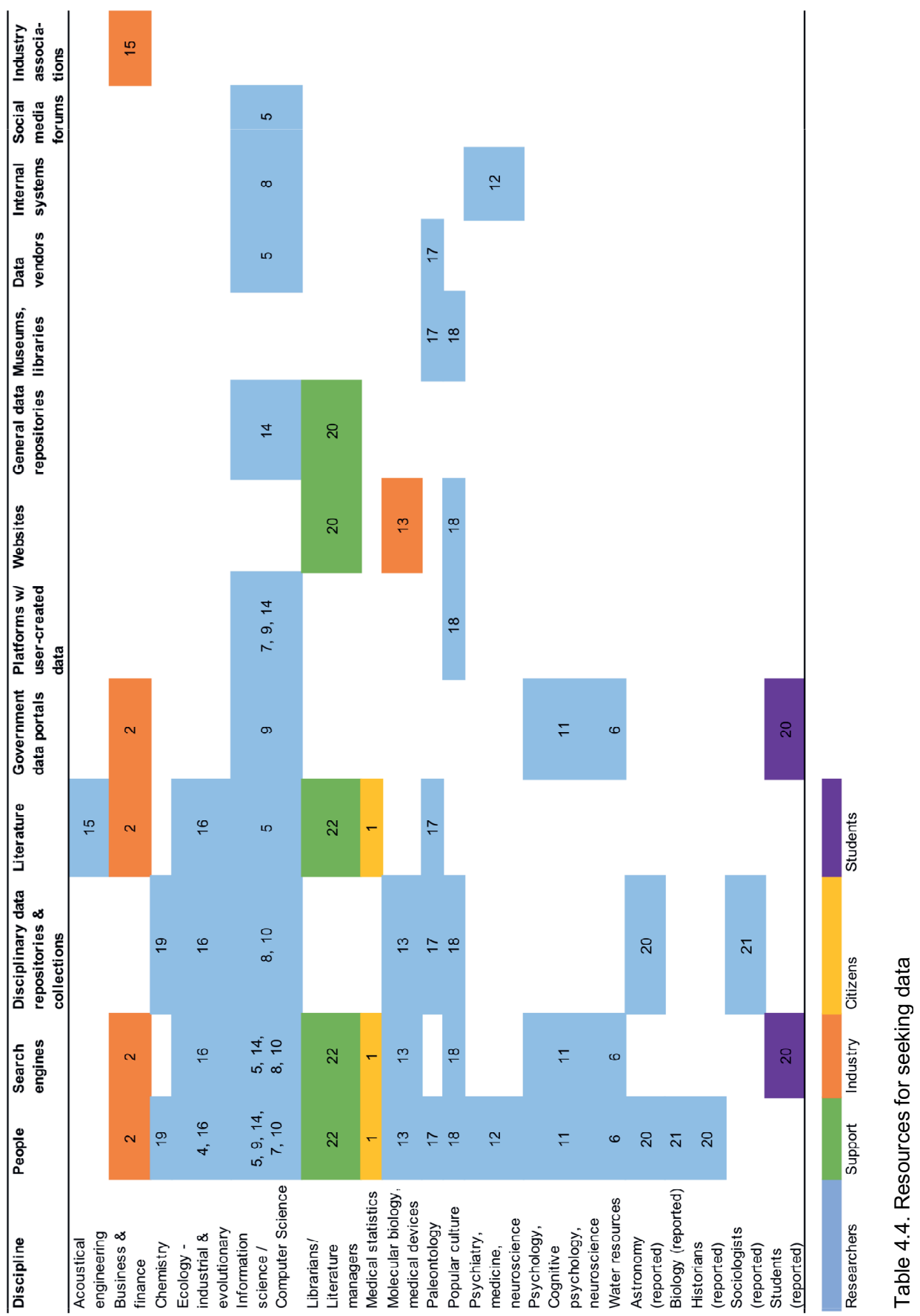




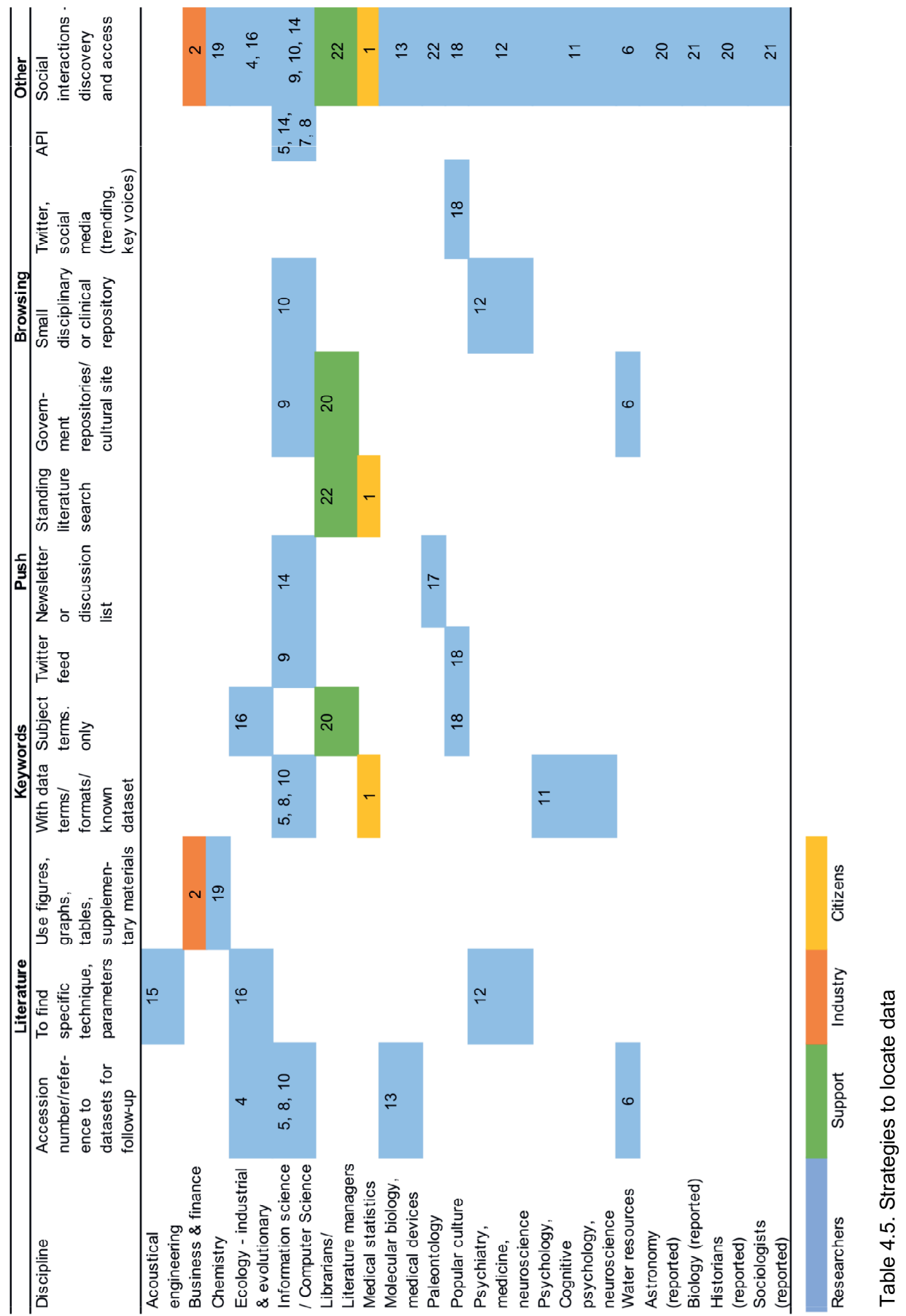


Disciplinary data repositories are important sources. Certain resources are considered to be "gold standards" within disciplines. Researchers also discover resources as they create data management plans or share their own research data in repositories $(21,4)$.

Many individuals discover data serendipitously while reading the literature; fewer actively search for data by conducting literature searches. Those who do are outside academia (1, $22,2)$, see the literature as part of their data $(5,17)$ or only need values commonly reported in the literature $(15,16)$. When participants encounter data serendipitously in the literature, they follow up by contacting authors or using dataset accession numbers or titles to locate the dataset online.

Other search strategies (Table 4.5) include keyword searches (with and without data-specific terms) and browsing using metadata or graphical interfaces. Some participants feel limited by keyword-only search boxes and desire greater browsing capabilities to increase precision.

Participants also find data through push-based strategies such as saved literature searches, subscriptions to newsletters or discussion lists, and carefully constructed Twitter feeds.

Often these services are set up to meet other information needs, and participants discover data through them serendipitously $(9,14,17)$. Other participants create feeds with the explicit purpose of finding textual data or data in the literature $(18,22)$.

Computer and information scientists use application program interfaces (APIs) to efficiently gather large amounts of data. They envision searching capabilities that incorporate APIs and link with computational tools (8) and systems that proactively search for data and present them in new ways.

\section{Social interactions in finding data.}

Participants locate data from colleagues, collaborators, supervisors, data authors, and support staff both serendipitously and intentionally. Data are encountered serendipitously during conference presentations $(19,13,22)$, informal conversations with colleagues (3) or because of geographic proximity (21). Interdisciplinary networking and training events provide an opportunity to unexpectedly learn about others' data; some interactions lead to data reuse. 
After these events, there is a lot of time for networking, and we have seen collaborations starting... between the School of Computing, who do the (data) visualization, and Sociology, who create interesting datasets and are interested in visualizing their data (21).

Personal connections are the most efficient and accurate route to data search for some participants.

Actually, most of the times that I have looked for external data, it has been through (personal) connections (11).

The human network of contacts is still the best way to find the information you want, especially if it is a small group...that is the most powerful and accurate source of information that I use at this point. (17)

Support staff engage in dialogue with their patrons to more accurately locate needed data $(20,22)$. Researchers also seek input from colleagues to design efficient queries (14). Some participants educate colleagues about the limitations of databases and how to search effectively $(13,20,16)$.

Professional networks and connections are also key to accessing data once found. Access to medical records and images is only possible through hospital affiliations $(12,8)$. Data are viewed as a gift in some communities; access is only granted to a small trusted circle of colleagues $(20,2)$. Historians must develop close relationships with the family members of people they are researching in order to be given access to documents (20). In countries with developing digital infrastructures, personal connections can be the only way to access nondigital data.

In my country, you don't get all of the data online. Sometimes you need to do the personal approach with some people in the (governmental) agency or department...I went directly to the department and met the person in charge, and then it was easier (6).

Collaborations provide a safe way for researchers to share and access data $(3,7)$. If an industrial ecologist needs data collected by industry or from another discipline, he forms new collaborations (16). Collaborations developed to access data can help early career researchers to grow their professional networks $(4,6)$. 
Finding data through social connections has limitations. Researchers risk operating in "filter bubbles" by only seeking information within their network $(3,18)$. Some assume there is no valuable data outside of their circle (21). Even within one's own lab, one may not know the details of others' data (8). It can be especially difficult to know who to contact to obtain data when operating outside of one's area of expertise $(12,14)$.

\section{Success.}

Participants using literature searches to locate data are satisfied with their methods or cannot think of better ways to meet their needs $(1,5,15,16,22)$. Researchers seeking data in other ways also feel that their methods are sufficient $(11,6,4,7)$, and believe they must combine multiple strategies to be successful (6), especially when they have goals beyond locating data.

I think if there was a good search engine, then I could get the dataset directly. I would still get in touch with the data author anyway, both for social reasons - developing the network and eventual collaboration - and also because most of the times the metadata are not enough to really understand the biology behind the species (4).

If researchers cannot find the data they seek, they assume that they are not available online $(17,4)$. Some participants will make do with the data they find or will give up the search $(14$, $10,2)$. Others will create their own data, believing that the data they need do not exist ( 9 , 10).

Searching skills also affect success. Students are often unskilled at finding and evaluating data (20). Although experts are assumed to be highly skilled at finding data in their field, this is not always true (21).

\section{Not all data are findable.}

Metadata quality determines whether or not data are findable $(8,21)$. Even in disciplines with well-developed metadata standards, researchers do not always describe their data in ways that facilitate discovery $(8)$ or follow standardized sharing methods $(14,10)$. When researchers do follow best practices, discovery can be hindered by poor links between data and publications (8). When physical data are digitized, valuable information and metadata risks being lost $(17,21)$. 
Not everyone has access to the same data. Often data are proprietary, owned by pharmaceutical or industrial firms $(1,12,2)$ or only searchable via expensive databases (19). Certain disciplines do not have domain repositories (5); multidisciplinary data repositories do not provide the search capabilities for specialized data, e.g. medical images (8). Existing data resources are also restricted by errors due to human indexing (13), unindexed information (7), or "ghost datasets" that are no longer curated or accessible (17). Research teams in numerous disciplines have built homegrown data repositories, but these resources are often not comprehensive $(10,13)$. Similarly, museum collections are built through networks and donations; curators decide what is included (17). Numerical data are often buried within larger numerical datasets (12) or the literature (19). Data are also not available for all regions of the world (17); if they are, the level of detail is not as good for certain regions (6).

\section{Evaluation}

Evaluation is intertwined with data analysis and occurs throughout the data search process $(13,7,6)$. Participants work extensively with data to determine its fitness for purpose (7). They also bring together information from multiple sources and perspectives to build fuller understandings and identify errors and biases $(13,2)$.

\section{Social interactions in sensemaking.}

Participants seek out others to make sense of data, carefully choosing whom to contact. Some contact data authors directly $(4,5)$; others seek advice from colleagues $(17,7)$ or from carefully nurtured personal networks (2). Contacting experts is especially important when using data from outside of one's discipline (14). One participant initiates collaborations in order to make sure he has team members with the necessary data expertise.

I am used to working with experts from different areas of knowledge. For me it is usual to have partners with different expertise: biology, agronomy, economy...l know the language of LCA (life cycle assessment), not of electronics or agricultural biology. My limit is not the data that I cannot find, but people that can work with these data (16).

Data reuse or implementation in a new situation requires more than metadata and documentation $(7,6,4)$. While metadata and documentation may provide enough information for a paper (7) or for background information (4), dialogues with data creators are imperative in ensuring appropriate and efficient reuse. 
Participants also use a variety of information about the data in their evaluations (Table 4.6); these are combined with other strategies to build trust and establish data quality (Table 4.7).

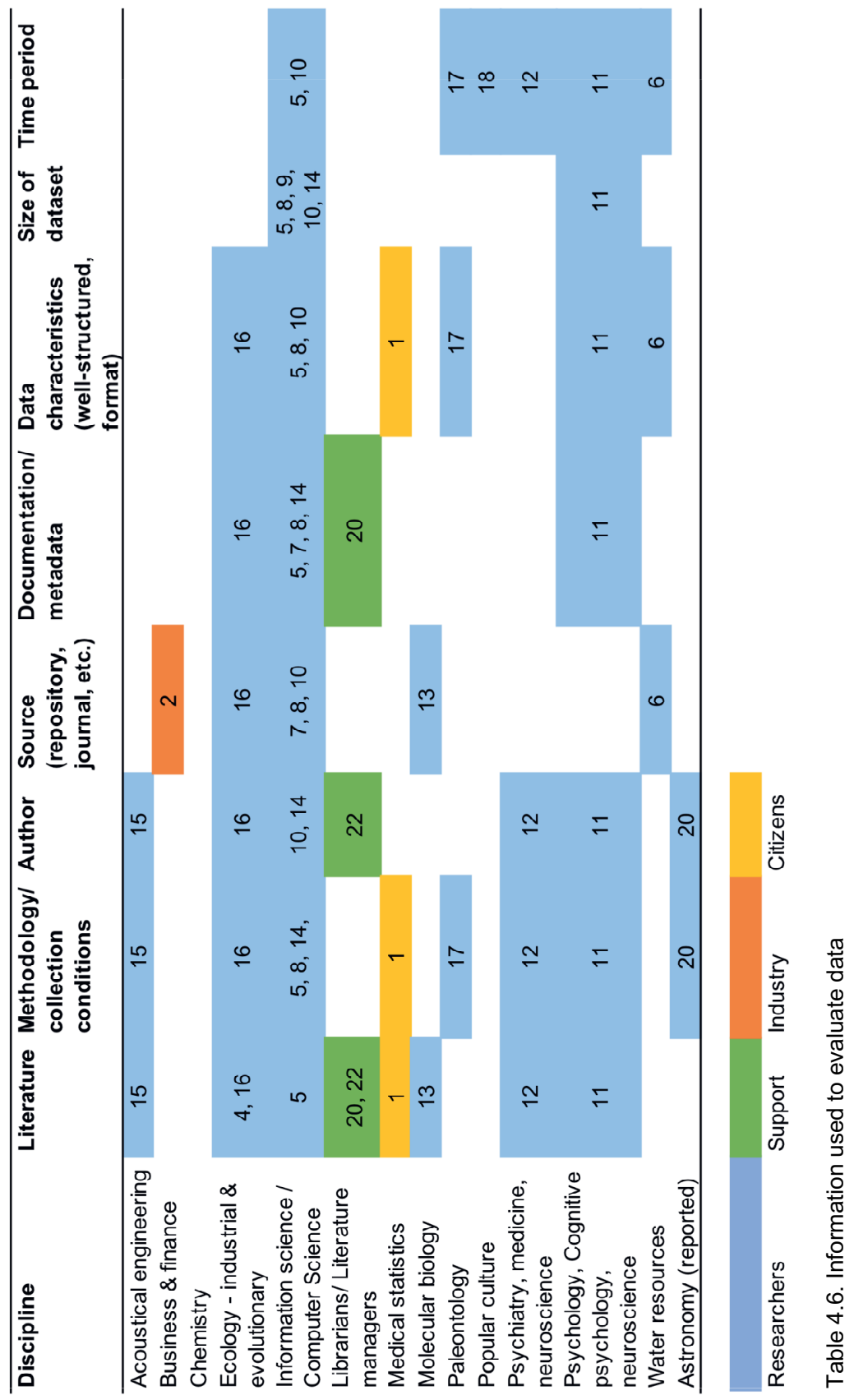




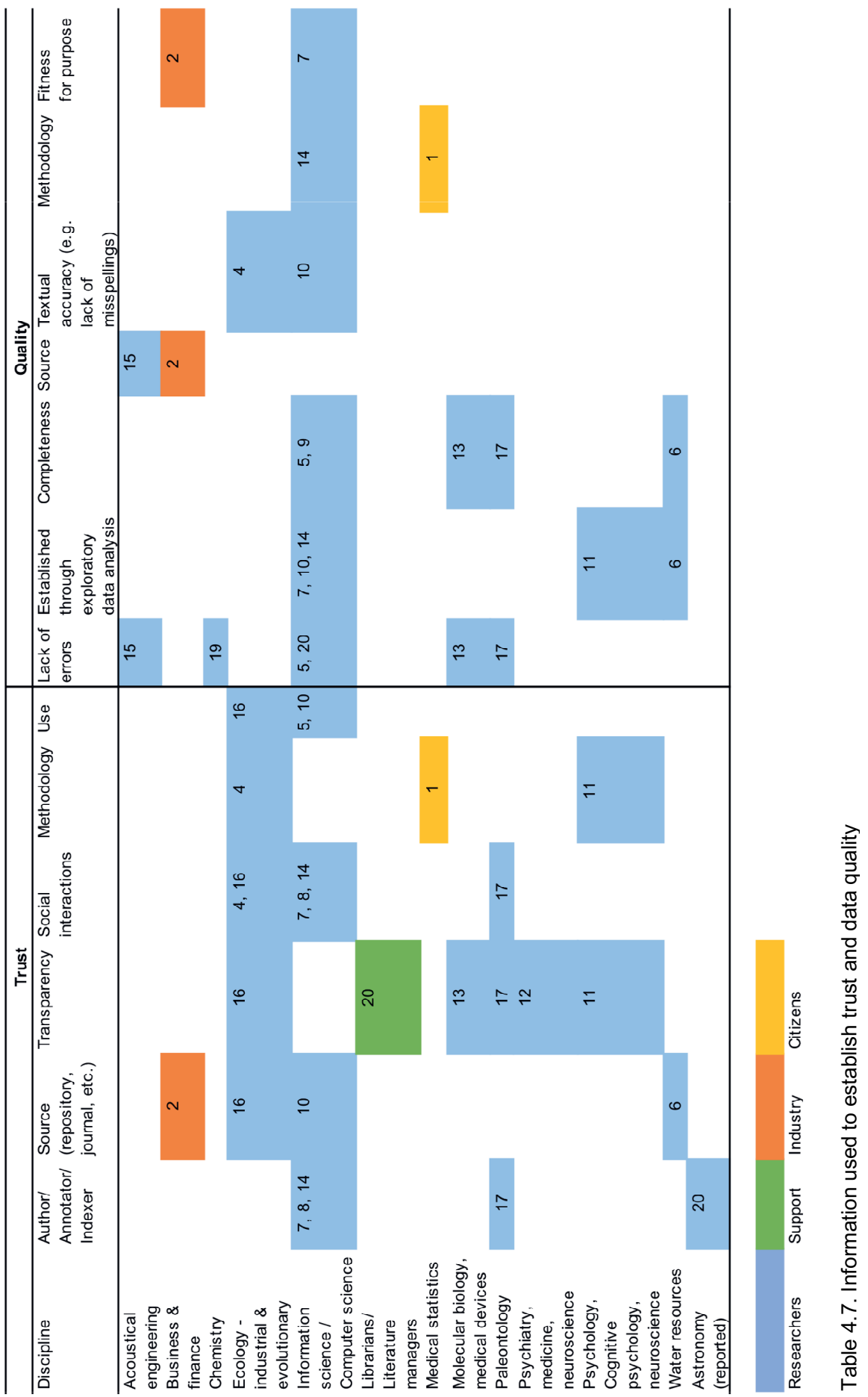




\section{Information used to evaluate data.}

The reputation, affiliation and size of repositories factor into reuse decisions. Some participants only use data from collaborators (7). Those in support roles rely heavily on the credibility of sources $(20,22)$; sometimes source information is the only evaluation criteria available to those outside of academia (2). The prolificacy (14), reputation $(15,22,11)$, and expertise (8) of the data author are important for some participants, but not for others $(1,5)$.

Participants require details about data collection and handling. They need information regarding environmental conditions (17), geographic coordinates (6), instrumentation and calibration (15), creation parameters $(15,16,14,4,8)$ and experimental protocols (4). Information about data provenance (14), processing (4) and statistical (1) or computational (20) tools is also used. Data must be well-structured, clearly labelled $(11,4)$, and in the desired format $(10,1)$ or resolution $(6)$.

Participants find the needed information in metadata, documentation and codebooks, and the literature. Individuals in support roles rely exclusively on these sources to decide which information to pass on to end users. One participant finds information solely through complex literature searching, as she does not trust herself to evaluate medical data.

I just would never trust myself enough; these are people's lives we are talking about. They (the customers) probably get more information than they need, but I think that it is helpful to them (22).

\section{Trust.}

We asked participants how they establish trust in secondary data. Some participants trust datasets commonly used in their disciplines or those used in peer-reviewed journals. Others need easy access to information about collection and analysis methods. Even small errors in the data or in the accompanying documentation will make researchers suspicious of the data (13). If a dataset is of sufficient size, worries about error are mitigated, as possible errors will be less significant. Errors can be identified by downloading and exploring data; if this process is facilitated, data are considered trustworthy (11).

The source of data, whether repository, journal, or governmental agency, is important in establishing trust. Social interactions with data owners provide a level of trust that cannot be easily replicated in an infrastructural system (17). Participants also consider the skill and reputation of the data annotator. This is used in conjunction with quality checks and 
knowledge of the data collection systems to develop trust (8).

Human and machine errors in indexing and information systems limit trustworthiness (13). Participants are aware that there is no such thing as a perfect dataset $(13,7)$, and that even seemingly trustworthy data may have been cleaned and presented in order to hide errors (4).

\section{Quality.}

We asked how participants think about data quality. Most associate data quality with minimal error; strategies to evaluate potential error include checking methodologies (1), choosing data in peer-reviewed journals $(15,16)$, or finding large datasets that minimize the effects of possible errors $(5,10)$. Participants also engage in exploratory data analysis, such as performing basic count checks (14); qualitative statistical checks (11) and calculating ratios between variables (e.g. precipitation to stream flow) (6) to evaluate quality. Completeness of metadata fields and coverage also indicates quality, although a certain level of incompleteness is to be expected in some cases $(13,7,6)$.

\subsection{Discussion}

Having presented empirical evidence about data search practices, we now synthesize and discuss our findings through a conceptual framework positing data seeking as a contextual, socio-technical practice. Examining our findings using this perspective, we see that both the data seeker and data themselves are often narrowly conceptualized, particularly by system designers. We follow this discussion with a proposal for how the theoretical points we develop could be transformed into practical considerations for system developers.

\subsubsection{A broader understanding of the user}

Both the literature study and interviews reveal that it is not enough to think of data users as researchers in a discipline with fixed practices. Communities, research interests, and practices are dynamic, at times influenced by the development of new research and analysis techniques (e.g. data science). Interdisciplinary projects create new communities and contexts, which necessitate new negotiations of data norms and blur disciplinary lines while enabling data discovery and reuse.

Not everyone who seeks data is a researcher. Librarians, literature managers, and students also seek data, using different strategies and evaluation methods. Individuals outside 
academia, including people working in industry and concerned citizens, are interested in finding and using data as well. The hint of another possible "user" is also emerging: the machine. As information retrieval systems develop to include proactive searching, some of the work currently done by humans may be automated in the future.

\subsubsection{A broader understanding of data}

Data needed for research are not always research data. Metadata, texts, server logs, device specifications, social media posts - all are used for foreground and background purposes in research but do not fall into what is traditionally thought of as "research data." This finding reflects the idea that people define data differently (Borgman, Wallis, Mayernik, et al., 2007), perhaps as a result of how they intend to use them. Applying our analytical perspective, it becomes possible to view data themselves as part of the dynamic ensemble of factors constituting context, with their very definition shaped by a user's intentions.

Data are also not always findable or reusable. Limitations in infrastructure, such as unstandardized metadata, un-curated datasets, or incomplete collections determine what data can be found, and thus reused. At the individual level, inconsistent data sharing practices, search abilities, social networks, and access rights hamper data search and reuse. Once data are found, there is no guarantee that users can access the resources (including humans) necessary to interpret and appropriately use the data.

While our findings support the idea of background and foreground data use (Wynholds et al., 2012; Wallis et al., 2013), we also find that data act as hubs for collaboration and creativity. Researchers form new collaborations in order to share, access and make sense of data. These collaborations help to grow professional networks and can inspire new approaches or future projects.

\subsubsection{Liminality}

Data are not static, of interest only to the community where they are produced. They move between situations and communities, existing in different contexts and being adapted to different purposes. The pathways that data travel also depend heavily on context - the context of their creation and the contexts of discovery and reuse.

While data search exists as an independent practice, it is also liminal, at times situated in other practices and spanning their thresholds. When users are engaged in data analysis, for example, they also negotiate meaning. When they manage or share their own data, they 
discover new resources. When they engage with their professional networks, they both find and make sense of data.

\subsubsection{Data search as a sociotechnical practice}

Some aspects of data seeking practices may seem clearly social, such as contacting authors or forming collaborations to access and understand data. Some aspects may seem clearly technical, such as retrieving data through an API or using exploratory data analysis.

The aim of socio-technical research is not to examine the social and the technical in isolation, but to examine the interactions that occur where the two intersect (Meyer, 2014). Applying this perspective to our findings, we see that data search practices are situated within and formed by interactions between the social and the technical spheres. For example, as users search for and evaluate data, they rely on metadata and documentation. Metadata schemas are created by humans; human practices and contexts also determine how those fields are populated. Users negotiate the social and technical worlds almost simultaneously, crossing the threshold between the two seamlessly, pointing again to the liminality of the process.

\subsection{Conclusion: Ramifications for system design}

Incorporating such theoretical understandings into the practical realm of system design can be valuable (Kling \& Star, 1997); we therefore conclude by suggesting the following points for designers of data discovery systems to consider, before highlighting areas for future work.

There is great variation in data sharing and description practices. Designers could engage with disciplinary communities and repository managers to improve metadata standardization.

Systems could also incorporate techniques for enriching metadata automatically or consider how to operationalize best practices such as the FAIR data principles, as do Doorn and colleagues (Doorn et al., 2017). Researchers find data in numerous repositories; discovery systems should therefore index both disciplinary and multidisciplinary repositories.

Data definitions and needs are changing. In order to support users' changing needs, systems could point to other data besides "research data." Disciplinary categories may also need rethinking to reflect the increasing interdisciplinarity of research. 
Given the variety of users, needs, and search preferences, systems should support keyword searching, browsing, and include an API. Differentiated search interfaces for user groups and support for students or disciplinary novices could also be implemented. Interactive maps, or "macroscopes," providing visual overviews of repository contents (Börner \& Record, 2017; Scharnhorst, 2015) could provide this support.

Users discover data serendipitously - either through networks or when searching for other information. They also find data when engaging in data sharing or data management. Systems could be designed for serendipitous discovery and be integrated with infrastructures supporting other data practices.

Social interactions are used to locate, evaluate and develop trust in data. Data themselves can facilitate new collaborations. Designing ways to contact data authors and ranking datasets via social signals could support social interactions. More speculatively, integrating offline and online interactions around data, including links to in-person training opportunities, would be worth investigating.

The inherent social nature of data search exceeds what can be implemented in a discovery system and needs to be addressed by various stakeholders, including policy makers. Policies and guidelines are often drafted from perspectives that bury the complexity and cultural specificity of data sharing and reuse (Borgman, 2015b; Tsoukala et al., 2016). While guidelines such as the FAIR data principles recognize the importance of making data findable, accessible, interoperable, and reusable, the importance of embedded social communication and the relevance of data practices in domain-specific epistemic processes, particularly in reusability, still need to be made explicit and deserve further study.

Our principal contributions in this work bridge areas that are often disconnected. First, our work provides an example of how scientometric studies can inform and shape qualitative research. This connection between the quantitative and qualitative is further strengthened in our tabular presentation of our interview data. We also present evidence that data search is a complex phenomenon grounded in the interplay between technology and social practices, but not reducible to either. Finally, we connect the theoretical and practical realms, suggesting how our findings could be implemented in system design.

More remains to be done. In particular, there is a need to further connect social and technical research by integrating broad query log analyses with in-depth case studies. 
Applying existing models of information seeking behaviors to examine data seeking behaviors could also offer a way to explore similarities and differences in practices, perhaps leading to new models describing data search practices.

\section{Acknowledgements}

KG developed the conceptual frameworks, collected and analyzed the data, and wrote the manuscript. HC, PG, AS, and SW contributed to theory development and editing. This work was funded by the Dutch Research Council (NWO), Grant 652.001.002. 
CHAPTER 5 


\section{Lost or found? Discovering data needed for research}

Originally published as: Gregory, K., Groth, P., Scharnhorst, A., \& Wyatt, S. (2020). Lost or Found? Discovering Data Needed for Research. Harvard Data Science Review.

https://doi.org/10.1162/99608f92.e38165eb 


\section{Abstract}

Finding data is a necessary precursor to being able to reuse data, although relatively little large-scale empirical evidence exists about how researchers discover, make sense of and (re)use data for research. This study presents evidence from the largest known survey investigating how researchers discover and use data that they do not create themselves. We examine the data needs and discovery strategies of respondents, propose a typology for data reuse and probe the role of social interactions and literature search in data discovery. We consider how data communities can be conceptualized according to data uses and propose practical applications of our findings for designers of data discovery systems and repositories. Specifically, we consider how to design for a diversity of practices, how communities of use can serve as an entry point for design and the role of metadata in supporting both sensemaking and social interactions. 


\subsection{Introduction}

Stakeholders from funders to researchers are increasingly concerned with the sharing and reuse of research data (e.g. Digital Curation Centre, n.d.; Tenopir et al., 2015). Policy makers draft guidelines, systems designers create repositories and tools, and librarians develop training materials to encourage opening and sharing data, often without empirical evidence about community-specific practices (Noorman et al., 2018). It is assumed that data can and will be reused if they are shared (Borgman, 2015b). Another assumption predicating reuse is that data will actually be discovered by researchers, although relatively little empirical work exists to support this assumption.

In this article, we present the results of the largest known survey examining how researchers discover and (re)use research data that they do not create themselves, so-called secondary data (Allen, 2017). We consider commonalities in practices but also examine differences, looking at how data needs and search practices vary not only by disciplinary domain but also by types of data uses. Past work has explored data search practices via in-depth interviews (Koesten et al., 2017; Borgman, Scharnhorst, \& Golshan, 2019; Gregory, Cousijn, et al., 2019). This study employs a broader approach, using a globally distributed multidisciplinary survey, with nearly 1,700 respondents, to explore these practices at a larger scale.

Our study produced a rich dataset including both qualitative and quantitative data. Here, we present the discovery phase of exploring the quantitative data, relying on descriptive statistics and tests for pairwise correlations, and we draw deeply on the qualitative responses. We use these analyses to paint a detailed picture of data discovery and propose a typology for data (re)use which we use to explore the data needs and practices of participants. We also probe the role of social interactions in searching for data and explore how data search is related to other practices, such as searching for academic literature. We consider how communities of data seekers can be conceptualized and discuss our findings in light of recent efforts to increase the discoverability of research data in a theoretical discussion. We conclude with practical considerations for the application of our findings by data discovery systems designers and repository managers and suggest further directions for research.

\subsection{Background}

Although information seeking is an extensive research field, work investigating data-seeking practices is nascent. Practices of data seeking, which we refer to here also as data search or 
data discovery practices, are commonly examined through user studies of particular data platforms and repositories (e.g. Borgman, Scharnhorst \& Golshan, 2019; Murillo, 2015; Wu et al., 2019). Zimmerman investigates data search practices directly, looking at the needs, discovery strategies, and criteria for evaluating data for reuse for a small group of environmental scientists. $(2003,2007,2008)$. Recent work characterizes data search and evaluation practices across disciplinary domains (Gregory, Groth, et al., 2019; Gregory, Cousijn, et al., 2019) and by data professionals within and outside of academia (Koesten et al., 2017), relying primarily on in-depth interviews with data seekers or log analyses (Kacprzak et al., 2017).

Surveys investigating data practices tend to use quantitative methods and focus on data sharing behaviors across disciplines (e.g. Tenopir et al., 2011; Tenopir et al., 2015; Kim \& Zhang, 2015), within specific domains (Tenopir et al.,2018; Schmidt et al., 2016) or in different geographic locations (Ünal et al., 2019). Publishers and data repositories conduct annual surveys tracing data sharing and management practices over time (e.g. Digital Science et al., 2018; Berghmans et al., 2017). Information about data search strategies, criteria important for reuse, and the role of social communications is found within surveys designed to develop data metrics (Kratz \& Strasser, 2015) and to determine factors affecting data reuse (Kim \& Yoon, 2017; Yoon, 2017b).

Interest in designing tools specifically for data search is growing (Chapman et al., 2020), evidenced by the development of search engines for research data (Noy et al., 2019; Scerri et al., 2017). Despite this trend, the limited amount of user interaction data restricts how these search tools are developed (Noy et al., 2019). There are also a growing number of policies regulating open data and data sharing (European Commission, 2019), which are seen as precursors to creating the ecosystem necessary for data discovery (Borgman, 2015a). These policies often do not accurately reflect the way that opening data and data sharing are enacted within communities (Noorman et al., 2018).

The sustainability and adoption of both search systems and data policies rely on understanding and building on extant practices (see Schatzki et al., 2001). Our work aims to provide evidence of practices of data seeking and to inform the design of community-centric solutions and policies. To do this we take a broad approach, looking for commonalities which can be used for design, while also highlighting differences. We present a detailed examination of the behaviors of our respondents as they engage in discovering, evaluating and reusing data; this work also provides a starting point for future analyses. 


\subsection{Methodology}

\subsubsection{Survey design}

We drew heavily on the findings of our earlier interviews with data seekers (Gregory, Cousijn, et al., 2019) and our analytical literature review (Gregory, Groth, et al., 2019) to design a survey addressing our principle research questions (see Table 5.1). Our research questions were informed by user-centered models of interactive information retrieval (e.g. Ingwersen, 1992; 1996; Belkin, 1993; 1996), particularly the synthesized model of an information journey proposed by Blandford and colleagues (Blandford \& Attfield, 2010; Adams \& Blandford, 2005) which generally posits an actor/user with an (at times unrecognized) information need who engages in an iterative process of discovery, evaluation and use.

Our survey employed a branching design consisting of a maximum of 28 individual items; nine of these items were constructed to allow for multiple responses. In addition to write-in responses for expanding on "other" answers, the survey included three open-response questions. Respondents working as librarians or in research/data support also answered a slightly modified version of the survey. ${ }^{6}$ Although we include some data from this group of "research support professionals" in the results presented here, most notably in Figure 5.10, the primary focus of this article is on researchers.

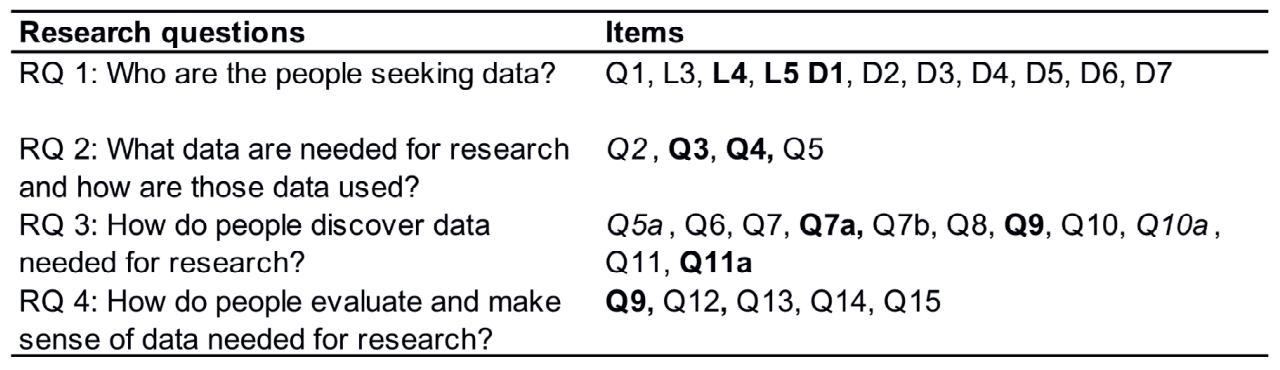

Table 5.1. Survey questions addressed by each research question. "L" questions were asked only to librarians/research support professionals; "D" questions were assigned to the demographics section. Multiple response questions indicated in bold; open response questions in italics.

\footnotetext{
${ }^{6}$ The survey instrument is available in the supplementary material for this article.
} 
Four multiple response questions and their associated variables are of particular importance in our analysis; we include an overview of these variables to aid in navigating our results (Table 5.2).

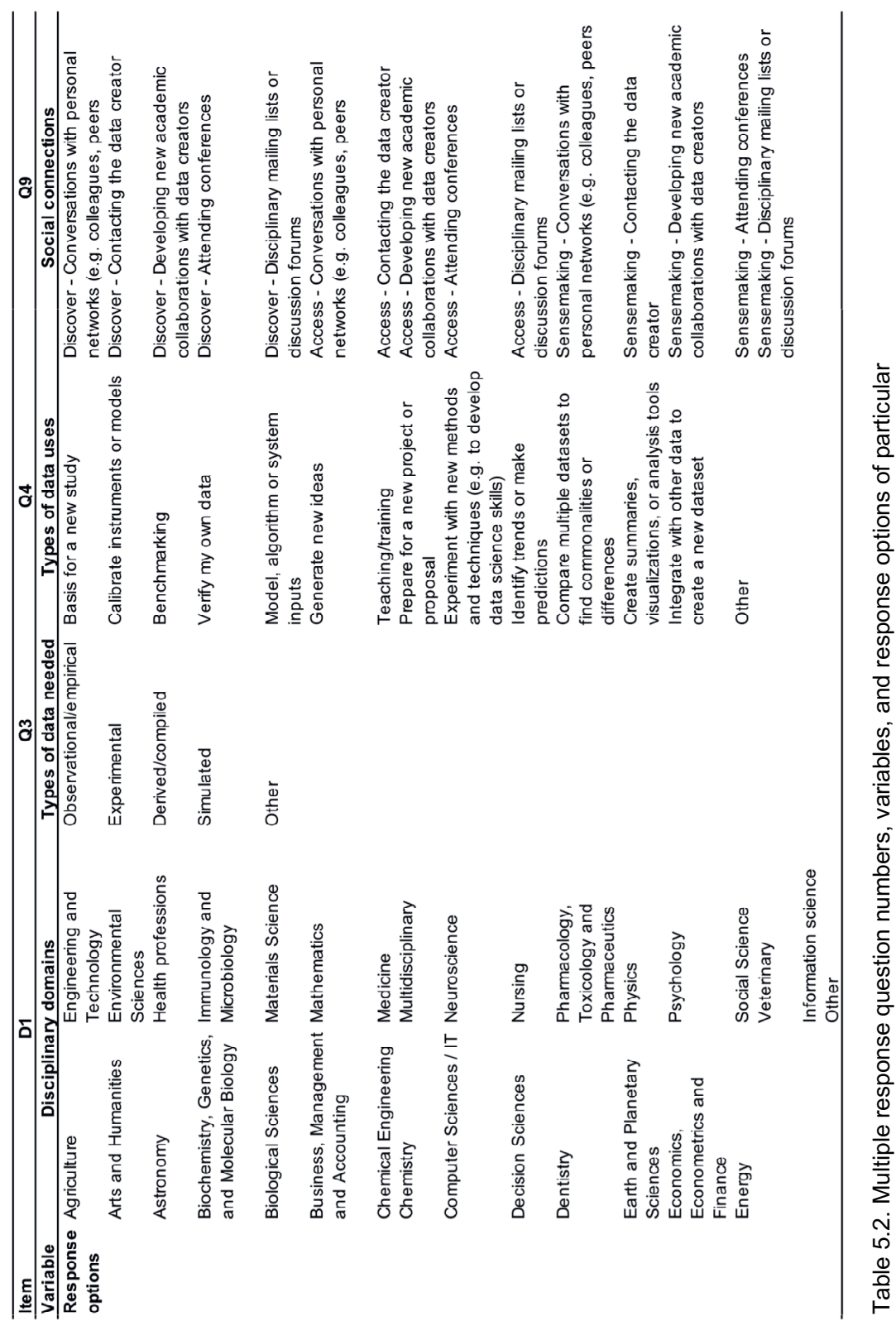


The survey was scripted and administered with the Confirmit software. ${ }^{7}$ We piloted the survey instrument in two phases. We scheduled appointments with four researchers, recruited via convenience sampling, and observed them as they completed the online survey. During these observations, we encouraged participants to think out-loud and ask questions. We used these comments to modify our questions before the next pilot phase. We then recruited an initial sample of 10,000 participants (using the recruitment methodology detailed below). Once one hundred participants had begun the survey, we measured the overall completion rate $(41 \%)$, taking note of the points in the survey where people stopped completing questions. We used this information to further streamline the question order and to clarify the wording of some questions before recruiting our sample. A demographic analysis of the non-complete responses was not possible, as demographic information was collected at the end of the survey questionnaire.

\subsubsection{Sampling and recruitment}

Our population of interest consisted of individuals involved in research, in multiple disciplinary domains, who seek and use data they do not create themselves. This is a challenging population to target specifically, as information about who seeks and reuses data, particularly in a diversity of disciplines, is difficult to isolate (i.e. Yoon, 2014a; Park et al., 2018). We therefore recruited our sample from a very broad population of researchers, hoping to capture a diverse sample of data seekers.

We sent recruitment emails to a random sample of an additional 150,000 authors who are indexed in Elsevier's Scopus database and who have published in the past three years. The recruitment sample was constructed to mirror the distribution of published authors by country within Scopus. Recruitment emails were sent in two batches, one of 100,000 and the other of 50,000 , two weeks after the first batch. One reminder email was sent to encourage participation. A member of the Elsevier Research and Academic Relations team created the sample and sent the recruitment letter, as access to the authors' email addresses was restricted. Potential participants were informed that the purpose of the survey was to investigate data discovery practices. We therefore assume that participants who completed the survey are in fact data seekers.

We received 1637 complete responses during a four-week survey period in SeptemberOctober 2018. We recruited an additional 40 participants by posting to discussion lists in the library and research data management communities, for a total sample of 1677 complete

\footnotetext{
${ }^{7}$ https://www.confirmit.com
} 
responses, yielding a response rate of $1.1 \%$. This response rate is calculated using the total number of recruitment emails distributed. It is likely that not all 150,000 individuals receiving recruitment emails match our desired population of individuals who search for and reuse data.

Of the recruited participants, 2,306 individuals clicked on the link to the survey, but did not complete it. Forty-two percent of people who engaged with the survey completed the questionnaire, similar to the completion rate in our pilot phase. Of the individuals who did not complete the survey, fifty percent viewed the introduction page with the informed consent statement, but did not click through to the survey itself. This could be because participants were not interested in the scope of the survey, had negative feelings about the funders or institutions involved, or that they did not agree with the information presented in the consent form. Seventy-five percent of individuals who did not complete the survey stopped responding by the fifth question, which was still within the first of the four sections of the survey.

\subsubsection{Analysis}

We used the statistical program $\mathrm{R}$ to perform our analysis, in particular the Multiple Response Categorical Variable (MRCV) package (Koziol \& Bilder, 2014a; Koziol \& Bilder, $2014 b$ ) to analyze questions with multiple possible responses. For these questions, we tested for multiple marginal independence (MMI) or simultaneous pairwise marginal independence (SPMI) between variables using the Bonferroni correction method. This method calculates a Bonferroni-adjusted p-value (Dunn, 1961) for each possible 2x2 contingency table that can be constructed from a question's variables. These individually adjusted $p$-values are then used to create an overall adjusted $p$-value which indicates if $\mathrm{MMI}$ or SPMI exist; specific associations between variables can then be identified by comparing the $p$-values from the individual $2 \times 2$ tables to the set significance level $(\boldsymbol{\alpha}=0.05)$ (Bilder, \& Loughin, 2015). This method was used to detect the correlations presented in Figure 5.6, Figure 5.8, Figure 5.15 and Table 5.4. This test can produce overly conservative results, particularly when analyzing questions with many variables. Nonetheless, this approach is preferred to traditional tests for independence, as it takes into consideration the fact that a single individual can contribute to multiple counts within a contingency table. We coded and analyzed open response questions in NVivo using a combined deductive and general inductive approach to thematic analysis (Thomas, 2006) and used R, Gephi and Tableau to create plots and visualizations. 


\subsubsection{Reporting}

Significant associations between variables are often reported by listing $p$-values in tabular format for each combination of possible responses. Due to the complexity of our questionnaire, in particular the number of multiple response variables (Table 5.2), we present significant associations using visualizations. These visualizations indicate if an association between variables exists; they do not indicate the value of the p-values themselves. We do this in order to increase the understandability of our results and to make them usable for a wider audience. Tables with the adjusted $p$-values are included in the supplementary material for this article.

\subsubsection{Limitations}

The sampling methods, the survey design, and our analytical methods have both strengths and limitations (see Box 5.1). Our data and analysis are descriptive, not predictive, and only represent the practices of our respondents - a group of data-aware people already active in data sharing and reuse and confident in their ability to respond to an English-language survey. We also have limited knowledge about individuals who did not respond to the survey. The analysis presented here is not generalizable to broader populations, but rather depicts the behaviors and attitudes of the approximately 1700 respondents to our survey. 


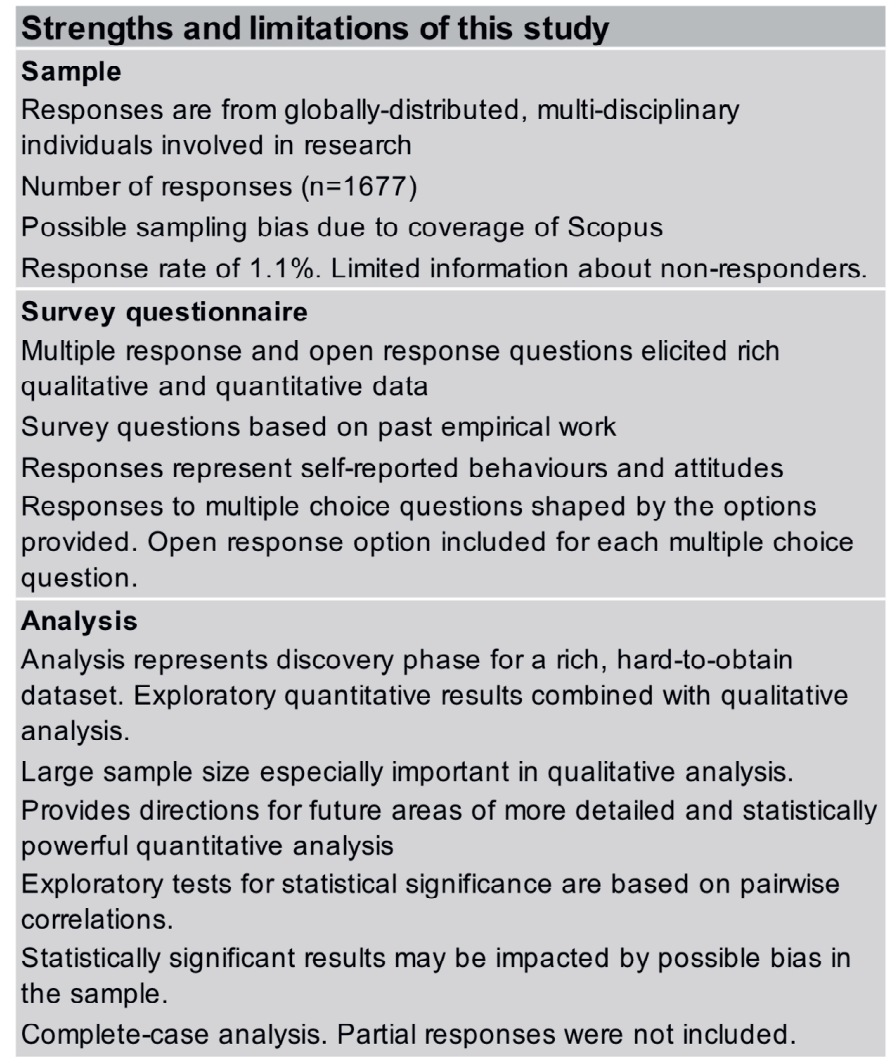

Box 5.1. Strengths and limitations of sampling, questionnaire design and analysis methods

The majority of our sample consists of researchers who have published in a journal indexed in the Scopus database. Certain disciplinary domains are better represented within Scopus; most notably the arts and humanities are not as well covered (Mongeon \& Paul-Hus, 2016; Vera-Baceta et al., 2019). Scopus has an extensive and well-delineated review process for journal inclusion; as of January 2020,30.4\% of titles in Scopus are from the health sciences; $15.4 \%$ from the life sciences; $28 \%$ from the physical sciences and $26.2 \%$ from the social sciences (Elsevier, 2020b). While the policies and content of Scopus could produce a potential bias in our sample, drawing from this population also helped us to target our desired population of researchers across domains.

Self-reported responses could be impacted by respondents' desire to give socially acceptable answers. Respondents may also have interpreted the survey questions in different ways; responses could be influenced by English-language fluency, individual interpretations of the wording of questions, the multiple choice options and Likert scales 
provided, and the ordering of the questions themselves. We attempted to counter these issues by implementing a two-stage pilot phase and by providing open response options for multiple choice questions.

Our findings are based only on complete survey responses. While this facilitated our analysis, it is possible that this introduced additional biases to our sample as well as reducing potential statistical power (as described in Pigott, 2001). The test for independence that we apply to identify correlations among questions with multiple response variables has been suggested to err on the conservative side, (Bilder \& Loughin, 2015), making it possible that some correlations were not detected with this method. The Bonferroni adjustment is an approximation; known issues include the identification of false negatives, particularly when comparing large numbers of variables, and determining the number of comparisons to use in the calculation (McDonald, 2014). For these reasons, we present our quantitative results as an initial exploration of the data, to guide deeper future statistical analyses.

\subsubsection{Ethics and data availability}

We received ethical approval from Maastricht University for the study. Participants had the opportunity to review the informed consent form prior to beginning the survey and indicated their consent by proceeding to the first page of questions.

The data from this survey are available in the DANS-EASY data repository under a CC-BY4.0 license (Gregory, 2020a).

\subsection{Results and analysis}

We present our results according to the research questions presented in Table 5.1. Each subheading provides an answer to the proceeding research question, which is then further discussed and supported by the survey data. The research questions are therefore answered in the course of the results section, before the primary takeaways are summarized in the Conclusion. We first examine characteristics of the data seekers responding to our survey, and then proceed to look at their data needs, search and discovery strategies, and evaluation and sensemaking practices.

\subsubsection{RQ 1: Who are the people seeking data?}

Respondents are globally distributed and have research experience 
Respondents employed in 105 countries completed the survey. The United States, Italy, Brazil and the United Kingdom were among the most represented countries (Figure 5.1a). This does not directly correspond with the sampling distribution (Figure 5.1b), where the largest difference between recruited participants and respondents occurred in China. This lower response rate could be due to language differences, perceived power differences (Harzing, 2006), or a lack of tradition in responding to research requests from researchers from other countries (Wang \& Saunders, 2012). It could also indicate that data seeking is not a common practice.

The majority of survey respondents were researchers (82\%) and worked in universities (69\%); $40 \%$ of respondents have been professionally active for $6-15$ years (Table 5.3 ). With the exception of participants recruited specifically from the library and research data management communities (or "research support professionals"), our recruitment methodology ensures that all respondents are published authors, making it likely that they have been involved in conducting research in either their past or current roles. Nearly half of those working in research support also need secondary data for their own research, in addition to supporting researchers or students.

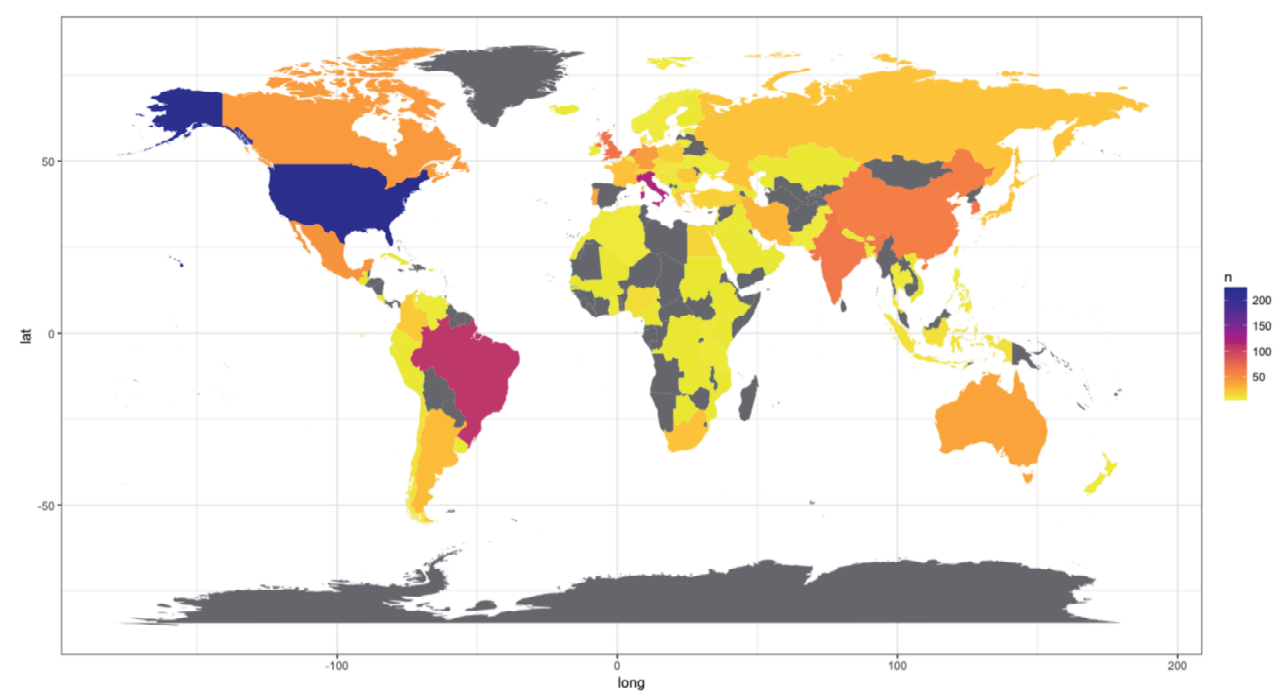

Figure 5.1. a) Number of respondents by country of employment ( $n=1677)$. 


\begin{tabular}{lc|lc}
\hline Percent of recruited sample & \multicolumn{2}{c}{ Percent of respondents } \\
\hline United States & $19 \%$ & United States & $13 \%$ \\
China & $15 \%$ & Italy & $7 \%$ \\
United Kingdom & $5 \%$ & Brazil & $7 \%$ \\
Germany & $5 \%$ & United Kingdom & $4 \%$ \\
Japan & $4 \%$ & India & $4 \%$ \\
France & $4 \%$ & South Korea & $4 \%$ \\
India & $4 \%$ & Netherlands & $4 \%$ \\
Italy & $3 \%$ & China & $4 \%$ \\
Canada & $3 \%$ & Mexico & $3 \%$ \\
Spain & $3 \%$ & Canada & $3 \%$ \\
Australia & $3 \%$ & Germany & $3 \%$ \\
South Korea & $3 \%$ & Australia & $2 \%$ \\
Brazil & $2 \%$ & Portugal & $2 \%$ \\
Netherlands & $2 \%$ & Iran & $2 \%$ \\
Russian Federation & $1 \%$ & Argentina & $2 \%$ \\
Taiwan & $1 \%$ & France & $2 \%$ \\
Iran & $1 \%$ & Greece & $1 \%$ \\
Swizerland & $1 \%$ & Japan & $1 \%$ \\
Turkey & $1 \%$ & Russian Federation & $1 \%$ \\
Poland & $1 \%$ & South Africa & $1 \%$ \\
Other & $19 \%$ & Romania & $1 \%$ \\
\hline
\end{tabular}

Figure $5.1 \mathrm{~b}$ ) Percent of recruited participants by country compared to percent of respondents by country $(n=1677)$

\begin{tabular}{lcc}
\hline Role & Number & Percent \\
\hline Researcher & 1372 & 82 \\
Student & 73 & 4 \\
Manager & 54 & 3 \\
Research support & 47 & 3 \\
Other & 131 & 8
\end{tabular}

\section{Organization}

\begin{tabular}{lcc} 
University & 1150 & 69 \\
Research institution & 287 & 17 \\
Government agency & 74 & 4 \\
Corporate & 68 & 4 \\
Independant archive & 2 & 0 \\
Other & 96 & 6 \\
Professional experience & & \\
$0-5$ & 206 & 12 \\
$6-15$ & 677 & 40 \\
$16-30$ & 502 & 30 \\
$31+$ & 292 & 17 \\
\hline
\end{tabular}

Table 5.3. Role, place of employment and years of professional experience of respondents $(n=1677)$. 


\section{Respondents support data sharing and reuse}

While eighty percent of all respondents reported sharing their own data in the past, participants with longer careers have done so slightly more often. Eighty-nine percent of respondents who have worked for $31+$ years reported having shared their data, compared to $77 \%$ percent of respondents working for less than five years. Personal attitudes towards data sharing and reuse differ from the perceived attitudes of peers, disciplinary communities and institutions (Figure 5.2). The majority of survey respondents are proponents of sharing their research data; they believe that the other groups identified in Figure 5.2 are less supportive of data sharing. This also points to a possible bias in our data, suggesting that people who have not shared their own data or who do not support data sharing were less likely to respond to our survey. Alternately, respondents could have felt compelled to provide a socially desirable answer, feeling a positive response was more acceptable. Respondents indicated that they believe data sharing is more strongly supported by their direct co-workers than by their disciplinary communities or institutions; they are most uncertain about the attitudes of their institutions. A similar pattern exists for attitudes toward data reuse, although there is more uncertainty involved.

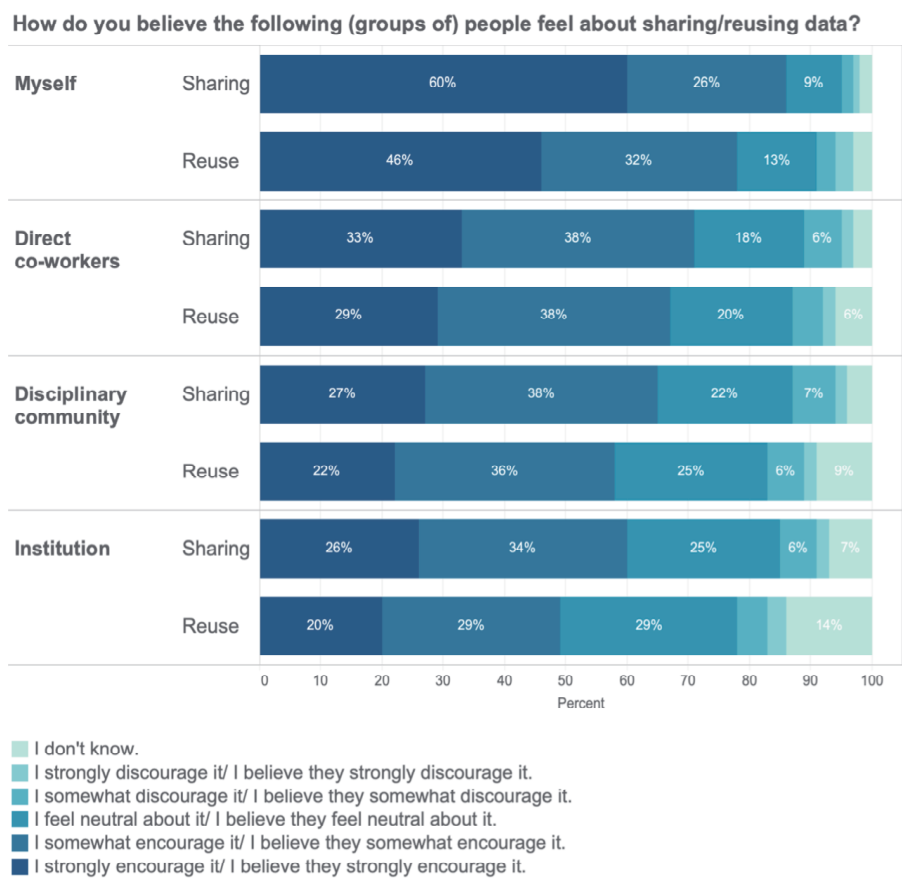

Figure 5.2. Respondents' beliefs about how they and other groups feel about data sharing and reuse $(n=1677)$. Percent denotes percentage of respondents. 


\section{Respondents belong to multiple, overlapping domains}

Respondents indicated their domain(s) of specialization from a list of 31 possibilities. This disciplinary list was originally used in a survey measuring data sharing practices across disciplines (Berghmans et al., 2017). Engineering and technology was selected most often, followed by biological, environmental, and social sciences (Figure 5.3). Approximately half of the respondents selected two or more domains, with one quarter selecting more than three. This could be a factor of varying levels of granularity of the disciplinary list; it could also indicate that participants found it challenging to choose a single domain that captures the complexity of their expertise.

Figure 5.3 depicts the disciplinary overlaps among respondents, showing which domains were selected in conjunction with each other. The figure reveals expected disciplinary overlaps, e.g. between information science and computer science, between medicine and health professions and between material science and chemistry. Other overlaps are perhaps less expected, for example between social science and engineering and technology or between arts and humanities and computer science, which could be indicative of the use of digital humanities methodologies among our participants. Seventy-one percent of respondents who selected engineering and technology chose at least one other discipline, most frequently computer science, environmental science, material science, and energy, as is visible in Figure 5.3. 
Dentist

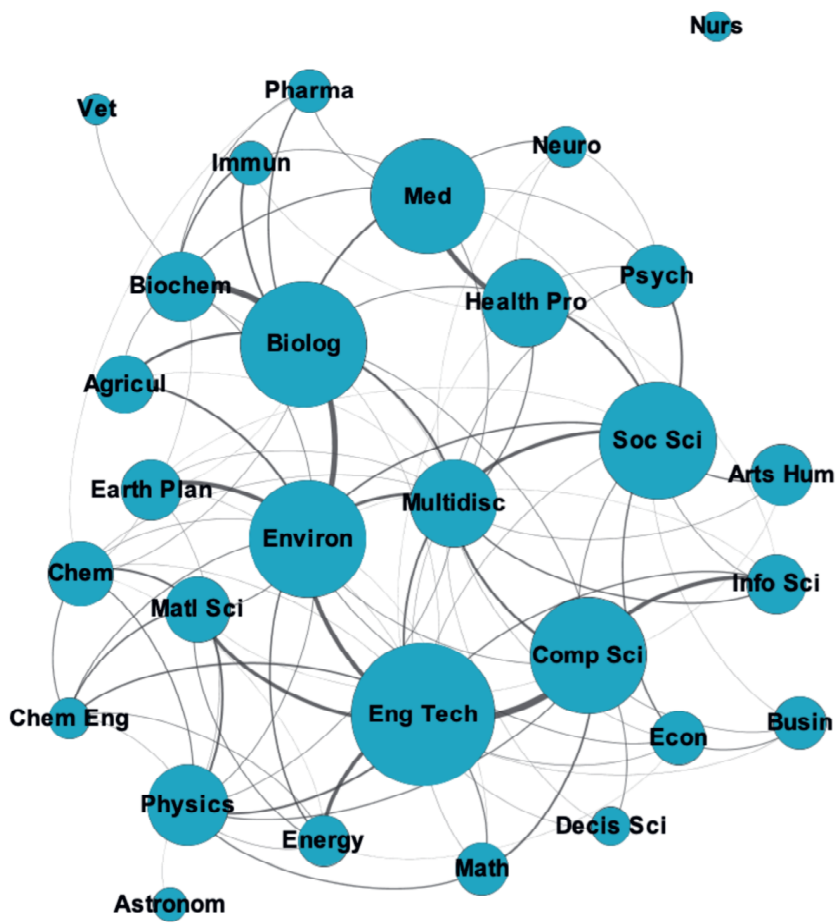

Figure 5.3. Disciplinary domain responses $(n=3431)$. Node size represents the number of responses from a discipline. Width of the edges represents the number of times two disciplines were selected in conjunction with each other. Only edges with a weight greater than 20 are shown; some edges pass behind other nodes. Graph created in Gephi; graph shape set using ForceAtlas algorithm, repulsion strength $=9000$; other parameters set to default.

We also identified individuals who selected only one discipline. The greatest number of single-discipline responses were in the domains of medicine, social science, engineering and technology and computer science (Figure 5.4). In future sections of this paper, we augment our analysis of respondents across all disciplinary domains with an occasional analysis of a subset of the individuals selecting only one discipline.

In this disciplinary subset, we included domains with more than 40 respondents, with the exception of the "other" category, as well as other domains whose data practices have been well-documented in the literature, i.e. astronomy, environmental sciences and earth and planetary sciences (see Gregory, Groth, et al., 2019 for a review of the literature exploring 
these disciplines). Disciplines which are included in this disciplinary subset are marked with a double asterisk in Figure 5.4.

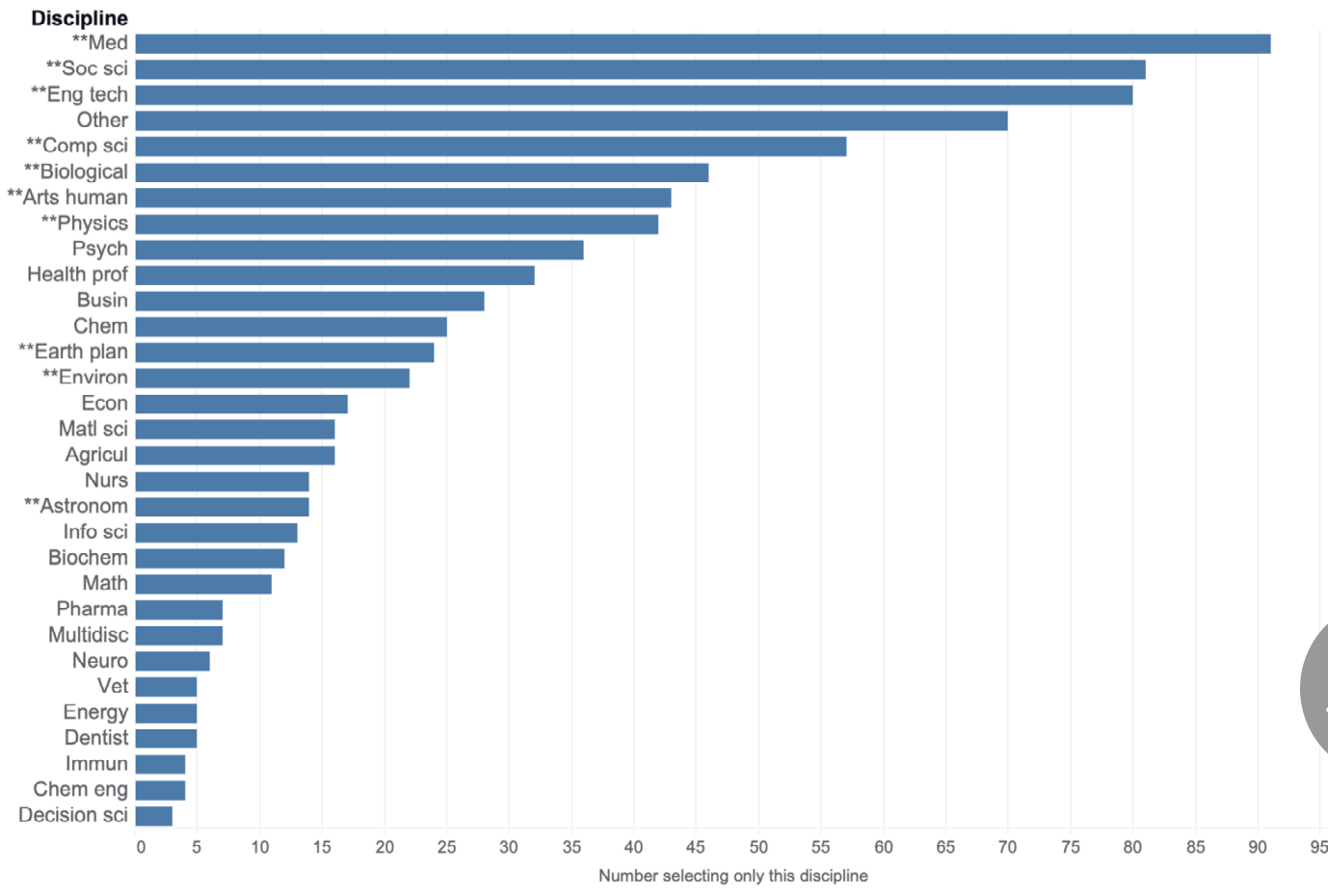

Figure 5.4. Number of respondents who selected only one discipline $(n=836)$. Disciplines included in the subset for further analysis marked with **.

\subsubsection{RQ 2: What data are needed for research and how are those data used?}

\section{Data needs are diverse and difficult to pigeonhole.}

To provide a high-level view of their data needs, respondents selected the type(s) of data that they need in their work from a list derived from the categories of research data proposed by the United States National Science Board (2005) and used in (Berghmans et al., 2017; Gregory, Groth, et al., 2019). While observational/empirical data were selected most frequently, $50 \%$ of participants also indicated that they need more than one type of data. Figure 5.5 represents the number of respondents selecting individual and multiple data types. 
Please select the options that describe the secondary data that you (might) need.

Observational or empirical (e.g. sensor data, survey data,

interview transcripts, sample data, neuroimages, ethnographic data, diaries)

Experimental (e.g. gene sequences, chromatograms, toroid magnetic field data)

$\square$ Simulation (e.g. climate models, economic models)

Derived or compiled (e.g. text and data mining, compiled database, 3D models)

$\square$ Other, Please specify

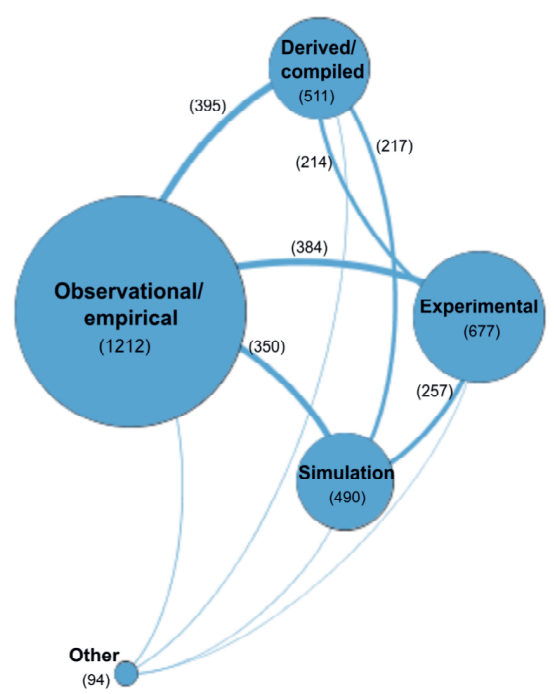

Figure 5.5. Question from survey with descriptions of data types. Node size in visualization represents number of responses for each data type $(n=2984)$. Edges represent number of times both data types were selected in conjunction with each other.

We asked participants to further expand on their data needs in an open response question, which seventy-eight percent of respondents completed. We compared these responses to the types of data that participants selected, paying special attention to those who chose multiple data categories. Respondents selecting multiple data types appear to do so for different reasons. Some require a variety of topic-specific data to conduct their research; others use a variety of data, regardless of topic, as long as it matches format and structure requirements. The following participant indicated needing observational, experimental and derived data.

[I need] large and small datasets that students can use for Data Science skill development. 
For example, transactional data from business, medical data such as interactions between patients and doctors, descriptive medical histories. Data need to be complex enough to be interesting and able to be parsed. (Respondent ID 613).

Data are difficult to categorize (Borgman, 2015a; Leonelli, 2019); in part because people may define data categories differently. While the majority of respondents stating they use census data selected observational data, others did not, choosing instead derived/compiled data. While the majority of individuals using literature corpora selected derived/compiled data, a minority selected "other," apparently not knowing which category best fit their data.

Associations (detected using the test for independence described in the Methodology section above) between disciplinary domain and the type of data needed are shown in Figure 5.6. The highest number of disciplinary associations were detected for experimental data; arts and humanities was associated with the greatest number of data types, although surprisingly not with observational data. There are also domains where no associations were detected. This could be due to the composition of our sample; it could also be taken as evidence for the diversity of data types that participants within disciplines need.

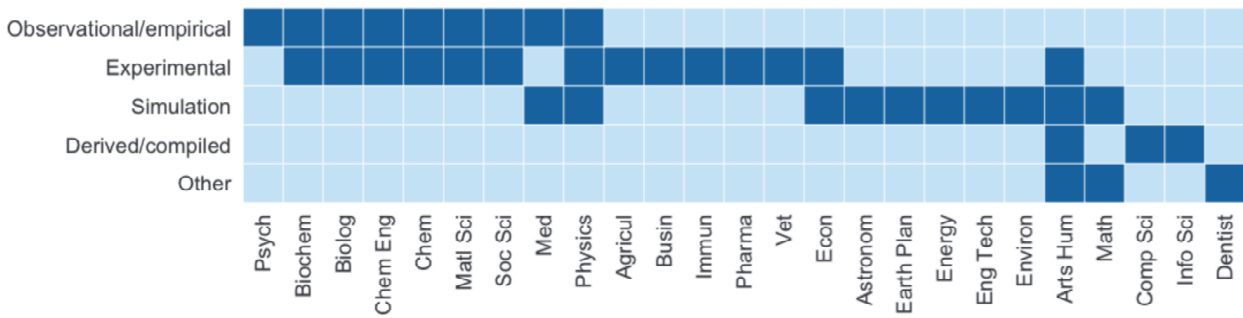

Figure 5.6. Associations between disciplinary domain and needed data. Associations detected using adjusted Bonferroni test for simultaneous pairwise marginal independence $(n=1677$; significance level: $p<0.05)$.

Slightly more than half of respondents reported needing data outside of their disciplines. This pattern holds across domains, with the exception of environmental science, where more individuals need data external to their domain (65\%), and medicine, where the trend was reversed, with less than half needing this type of data (43\%). 


\section{Data uses are diverse but limited to a core set}

Although using data in ways that support research is well-documented (e.g. Wallis, Rolando, \& Borgman, 2013), using data to drive new research or in teaching is not as well documented (Gregory, Groth, et al., 2019). The majority of respondents (71\%) selected using data as the basis for a new study, which is in line with other very recent research (Pasquetto et al., 2019); half of respondents selected needing data to use in teaching (Figure 5.7). Other uses include experimenting with new methodologies and techniques such as developing data science skills or completing particular data-related tasks, i.e. trend identification or creating data summaries (as suggested by Koesten et al., 2017). Less than two percent of respondents indicated needing data for other purposes, suggesting that the list of uses in our survey is fairly complete.

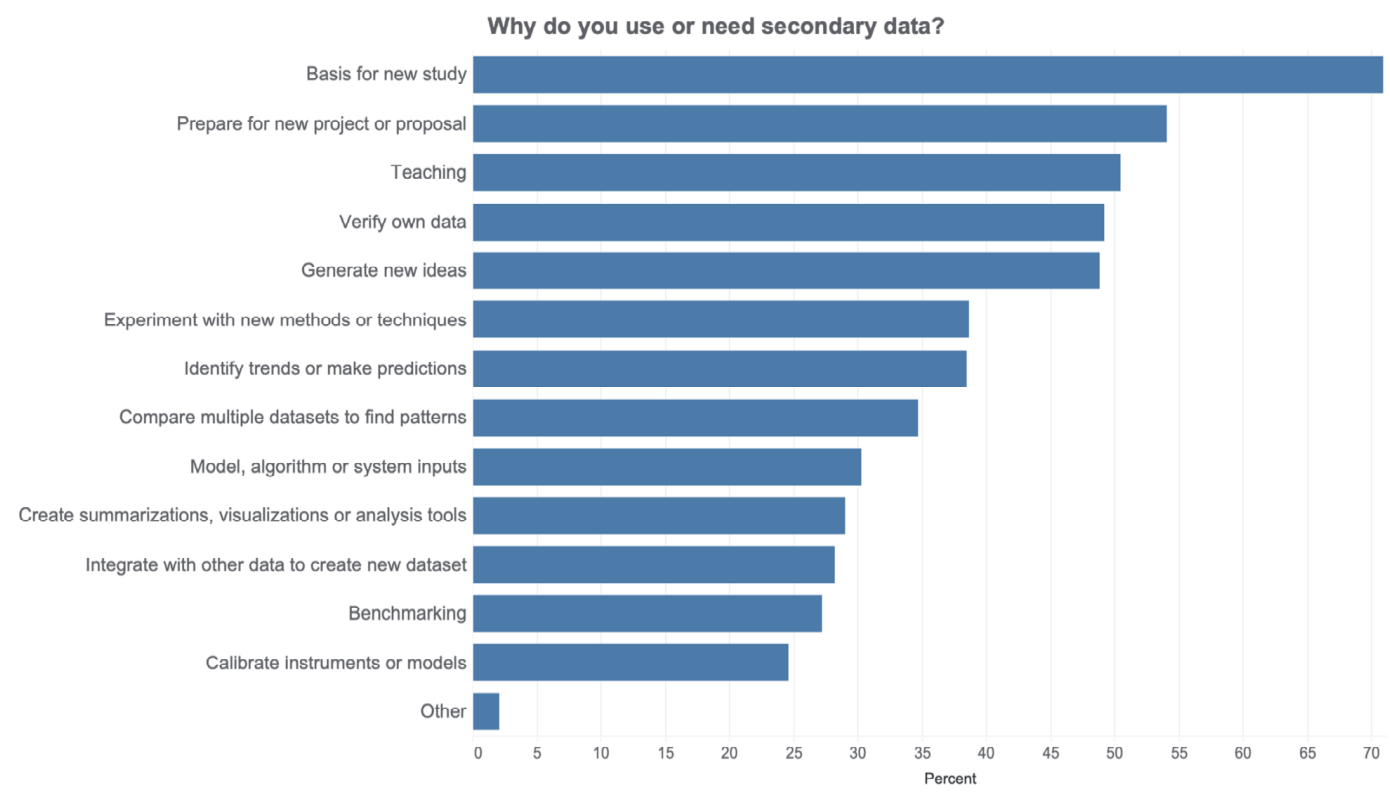

Figure 5.7. Reasons why respondents need secondary data. Multiple responses possible. Percent denotes percentage of respondents $(n=1677)$.

\section{Data uses span research phases}

Table 5.4 presents significant associations between the data uses shown in Figure 5.7 and the types of needed data shown in Figure 5.5. We also tested to see if significant associations exist between the different types of data uses (Table 5.4: Associated with these types of data uses). We recognize that associations between uses are difficult to interpret; if an individual selected teaching, i.e., it may or may not be related to their selection of another 
data use. To examine these associations through a different perspective, we organize them along a conceptual typology, which we propose here, based on different phases of research.

To create this typology, we drew on the associations we identified, existing literature, and extant models of research lifecycles. In Table 5.4, we agree with van de Sandt, DallmeierTiessen, Lavasa, \& Petras (2019) that reuse is one type of data use, but similar to Fear (2013), we view reuse as referring to using data as the basis for a new study. Our typology has similarities with models of the research lifecycle process (e.g. Jisc, 2013), but it also differs from these models. Typical models of research lifecycles portray research that uses data created by the researcher, rather than secondary data. They also tend to reduce the complexity of research processes (Borgman, 2019), ignore the interwoven nature of tasks involved in research (Cox \& Wan Ting Tam, 2018) and depict data cycles as independent workflows (e.g. UK Data Service, 2019). With this typology, we attempt to nuance the uses of secondary data throughout phases of research, recognizing how data uses are associated with multiple data types and uses and highlighting that these uses are associated with multiple work phases.

Depending on research practices, the data uses in our typology could occur in different phases. Uses that could particularly fall into two bordering categories are marked in grey. Integrating different datasets could occur when conducting research, e.g., or verification of data could also be considered to be part of data analysis. Although not marked in grey, data analysis and sensemaking tasks are likely to occur throughout all phases of research. This is reflected in the results, where analysis activities are associated with every other data use, with the exception of instrument or model calibration. 


\begin{tabular}{|c|c|c|c|}
\hline Research/work phase & Types of data uses & $\begin{array}{l}\text { Associated with these types of } \\
\text { needed data }\end{array}$ & $\begin{array}{l}\text { Associated with these types of } \\
\text { data uses }\end{array}$ \\
\hline Reuse & Basis for new study (new study) & observational/empirical & $\begin{array}{l}\text { new project, new ideas, } \\
\text { integration, comparison, trends, } \\
\text { teaching }\end{array}$ \\
\hline \multirow[t]{3}{*}{$\begin{array}{l}\text { Project creation and } \\
\text { preparation }\end{array}$} & $\begin{array}{l}\text { Prepare for new project or } \\
\text { proposal (new project) }\end{array}$ & & $\begin{array}{l}\text { new study, new ideas, integration, } \\
\text { new methods, verification, trends, } \\
\text { comparison, summaries/vis/tools, , } \\
\text { teaching }\end{array}$ \\
\hline & Generate new ideas (new ideas) & & $\begin{array}{l}\text { new study, new project, } \\
\text { integration, new methods, } \\
\text { verification, trends, comparison, } \\
\text { summaries/vis/tools, teaching }\end{array}$ \\
\hline & $\begin{array}{l}\text { Integrate with other data to create } \\
\text { new dataset (inlegration) }\end{array}$ & $\begin{array}{l}\text { observational/empirical, } \\
\text { derived/compiled }\end{array}$ & $\begin{array}{l}\text { new study, new project, new ideas, } \\
\text { new methods, inpuls, trends, } \\
\text { comparison, summaries/vis/tools }\end{array}$ \\
\hline \multirow[t]{5}{*}{ Conducting research } & $\begin{array}{l}\text { Experiment with new methods or } \\
\text { techniques - e.g. data science } \\
\text { skills (new methods) }\end{array}$ & $\begin{array}{l}\text { experimental, simulation, } \\
\text { derived/compiled }\end{array}$ & $\begin{array}{l}\text { new project, new ideas, } \\
\text { integration, inputs, calibration, } \\
\text { benchmarking, verification, trends, } \\
\text { comparison, summaries/vis/tools }\end{array}$ \\
\hline & $\begin{array}{l}\text { Model, algorithm or system inputs } \\
\text { (inputs) }\end{array}$ & simulation, derived/compiled & $\begin{array}{l}\text { integration, new methods, } \\
\text { calibration, benchmarking, } \\
\text { verification, trends, comparison } \\
\text { summaries/vis/tools }\end{array}$ \\
\hline & $\begin{array}{l}\text { Calibrate instruments or models } \\
\text { (calibration) }\end{array}$ & $\begin{array}{l}\text { experimental, simulation, } \\
\text { derived/compiled }\end{array}$ & $\begin{array}{l}\text { new methods, benchmarking, } \\
\text { inputs, verification }\end{array}$ \\
\hline & Benchmarking (benchmarking) & simulation, derived/compiled & $\begin{array}{l}\text { new methods, inputs, calibration, } \\
\text { trends, summaries/vis/tools }\end{array}$ \\
\hline & Verify own data (verification) & $\begin{array}{l}\text { experimental, simulation, } \\
\text { derived/compiled }\end{array}$ & $\begin{array}{l}\text { new project, new ideas, new } \\
\text { methods, calibration, } \\
\text { benchmarking, comparison, } \\
\text { teaching }\end{array}$ \\
\hline \multirow[t]{3}{*}{$\begin{array}{l}\text { Data analysis and } \\
\text { sensemaking }\end{array}$} & $\begin{array}{l}\text { Identify trends and make } \\
\text { predictions (trends) }\end{array}$ & $\begin{array}{l}\text { observational/empirical, simulation, } \\
\text { derived/compiled }\end{array}$ & $\begin{array}{l}\text { new study, new project, new ideas, } \\
\text { integration, new methods, inputs, } \\
\text { benchmarking, comparison, } \\
\text { summaries/vis/tools }\end{array}$ \\
\hline & $\begin{array}{l}\text { Compare multiple datasets to find } \\
\text { commonalities or differences } \\
\text { (comparison) }\end{array}$ & experimental, derived/compiled & $\begin{array}{l}\text { new study, new project, new ideas, } \\
\text { integration, new methods, } \\
\text { inputs, verification, trends, } \\
\text { summaries/vis/tools, }\end{array}$ \\
\hline & $\begin{array}{l}\text { Create summaries, visualizations or } \\
\text { analysis tools (summaries/vis/tools) }\end{array}$ & $\begin{array}{l}\text { observational/empirical, } \\
\text { derived/compiled }\end{array}$ & $\begin{array}{l}\text { new project, new ideas, } \\
\text { integration, new methods, inputs, } \\
\text { benchmarking, trends, comparison, } \\
\text { teaching }\end{array}$ \\
\hline Teaching & Teaching/training (teaching) & observational/empirical & $\begin{array}{l}\text { new study, new ideas, new project, } \\
\text { verification, summaries/vis/tools }\end{array}$ \\
\hline
\end{tabular}

Table 5.4. Associations between types of data use, needed data type and other data uses. Grey areas represent uses that could fall within multiple bordering research phases. Colors correspond to research/work phases. Associations detected using adjusted Bonferroni test for simultaneous pairwise marginal independence (significance level: $p<0.05$ ).

Using data as the basis for a new study is associated in our results with needing observational data. Among our respondents, observational data are not significantly associated with the tasks in the "conducting research" phase, but they are associated with activities in both data analysis and with teaching. Experimental data are related to uses involved in conducting research, as well as to comparison. Derived data are associated with all activities in both the research process and data analysis/sensemaking phases; simulation data are primarily associated with conducting research. 
Using data as the basis for a new study is associated with uses such as project creation and preparation, data analysis and sensemaking, or teaching, but is not associated with any uses in the conducting research phase. Teaching is associated with only a few other uses, most of which fall within the project creation phase. Data integration is associated with activities across phases of research; calibration, however, is exclusively associated with other research process tasks.

\section{Data uses are common, but their enactments are complex}

Disciplinary domains also shape how data are used. Figure 5.8 indicates significant associations between disciplines and uses in our sample. Most of these associations are for data uses that fall within the conducting research phase of the above typology, particularly using data as inputs and for calibration, and domains that typically make use of modelling or computational research methods. Due to the large number of variables compared in Figure 5.8 and the limitations of our test for significance, these associations in particular represent initial results.

To complement the associations presented in Figure 5.8, we also looked at the subset of our data for individuals selecting only one discipline (indicated in Figure 5.4). Within these disciplinary groups, we also found that respondents use data for a variety of purposes, rather than for just one type of use. While researchers in multiple domains may use data for the same purposes, uses will be enacted in different ways and have different meanings in various disciplines and contexts (Borgman, 2015a, Leonelli, 2015). While 39\% of computer scientists in the disciplinary subset and $35 \%$ of those in the arts and humanities selected using data for verification, for example, the actual practice and the meaning of verifying data will be different in each of these disciplines. 


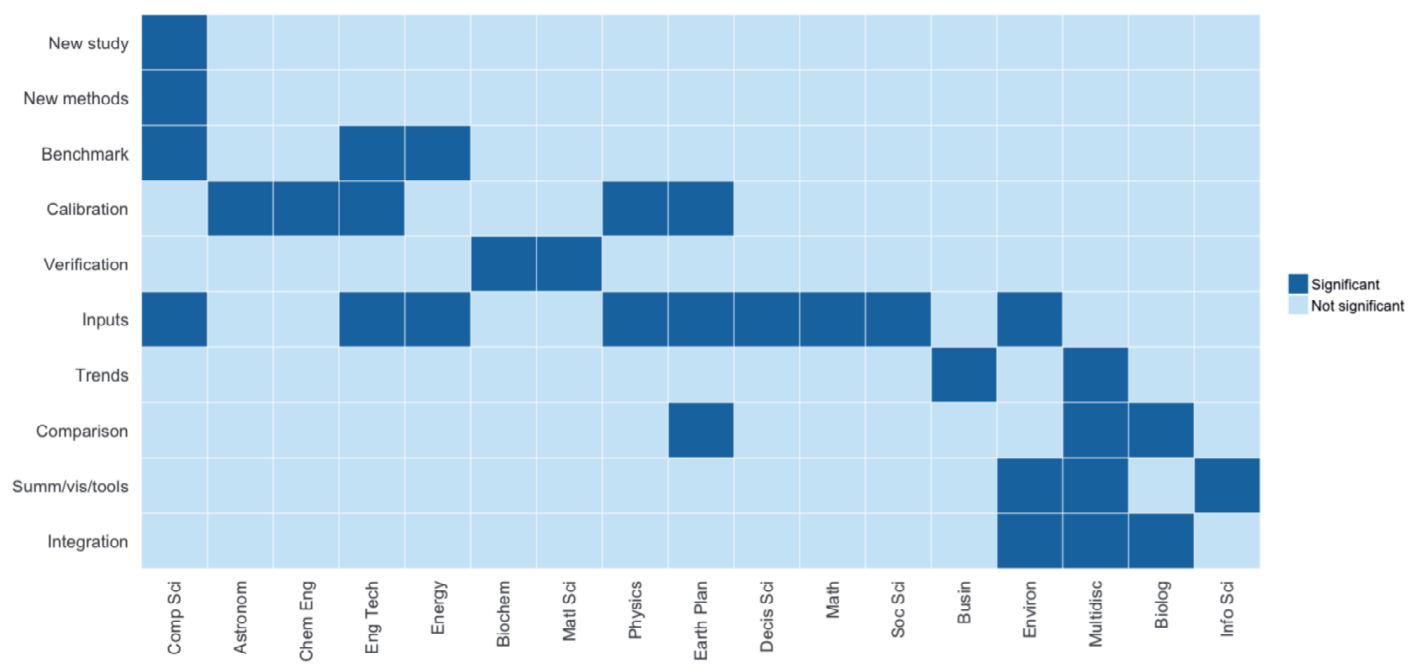

Figure 5.8. Associations between disciplinary domain and data use. Associations detected using adjusted Bonferroni test for simultaneous pairwise marginal independence $(n=1677$; significance level: $p<0.05)$.

\subsubsection{RQ 3: How are people discovering data?}

Researchers believe that discovering data is a sometimes challenging (73\%) or even difficult $(19 \%)$ task. The greatest challenge researchers face is that data are not accessible, i.e. data are not available for download or analysis, ( $68 \%$ of researcher respondents), followed by the fact that data are distributed across multiple locations (49\%). One third of these respondents identified inadequate search tools, a lack of skill in searching for data, or the fact that their needed data are not digital as being additional challenges.

\section{Via academic literature}

Thirty percent of respondents reported no difference in how they find literature and how they find data. Figure 5.9 presents the disciplines selected by these respondents. Some of these respondents chose multiple disciplines; the analysis in Figure 5.9 is therefore not limited to respondents in the disciplinary subset. Disciplines with data repositories that are closely linked with systems for searching the academic literature of that field, such as in the biomedical sciences and physics (i.e. the resources from the National Library of Medicine ${ }^{8}$

\footnotetext{
${ }^{8}$ https://www.ncbi.nlm.nih.gov/guide/all/
} 
and HEP-INSPIRE ${ }^{9}$ database, respectively) rank more highly. Respondents in disciplines where data repositories and academic literature databases are traditionally less integrated with each other, i.e. in the social sciences (e.g. the ICPSR ${ }^{10}$ database) or the environmental sciences (e.g. PANGAEA ${ }^{11}$ ), tend to have more distinct discovery practices.

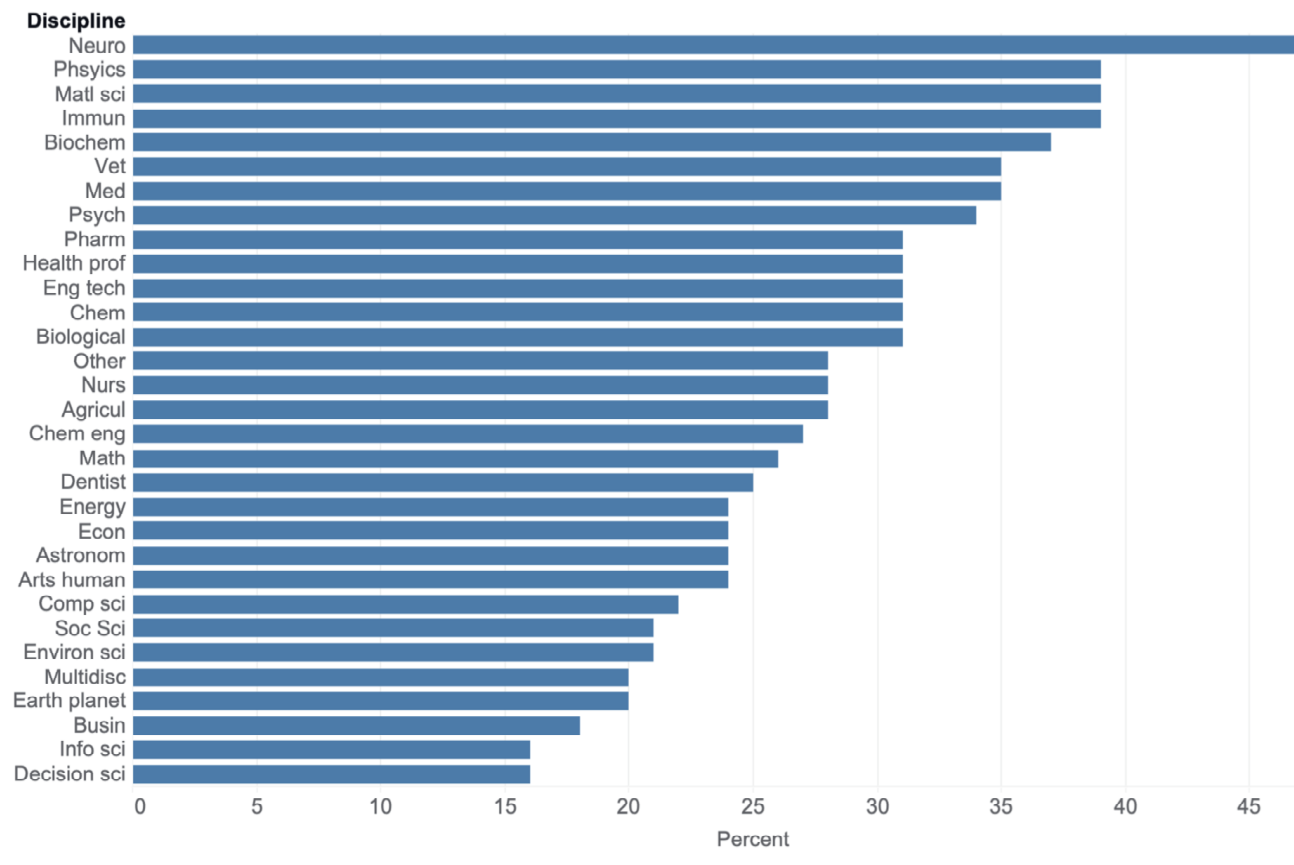

Figure 5.9. Percentage of responses $(n=959)$ by discipline for individuals reporting no difference in literature and data search strategies. Multiple responses possible.

Fifty-two percent of respondents stated that their processes for finding literature and data are sometimes the same and are sometimes different; whereas they were always different for $18 \%$. Respondents saying that the two processes are sometimes or always different $(n=$ 1178 ) were asked to explain the differences in an open response question.

One of the key differences participants identified between literature search and data discovery are the sources that are used.

\footnotetext{
${ }^{9}$ http://inspirehep.net

${ }^{10}$ https://www.icpsr.umich.edu/icpsrweb

${ }^{11}$ https://www.pangaea.de
} 
I check other channels for data than for literature, e.g. if a project produces data, I check the project's site directly for their data and hope for links to repositories.

(Respondent ID 4001)

Academic literature could be found through different portals...To receive data, one often needs to know where to find it. For example, the name on the database and then contact the administrator for the database if you can't extract the data directly from the database. (Respondent ID 4008)

Yet the academic literature itself is a key source for discovering data for researchers, as are general search engines (e.g. Google) and disciplinary data repositories (Figure 5.8).

Research support professionals rely less on the literature, more frequently turning to a variety of sources in their search for data (Figure 5.10). The importance of literature, search engines and domain repositories across disciplines supports the findings from earlier work (Kratz and Strasser, 2015).

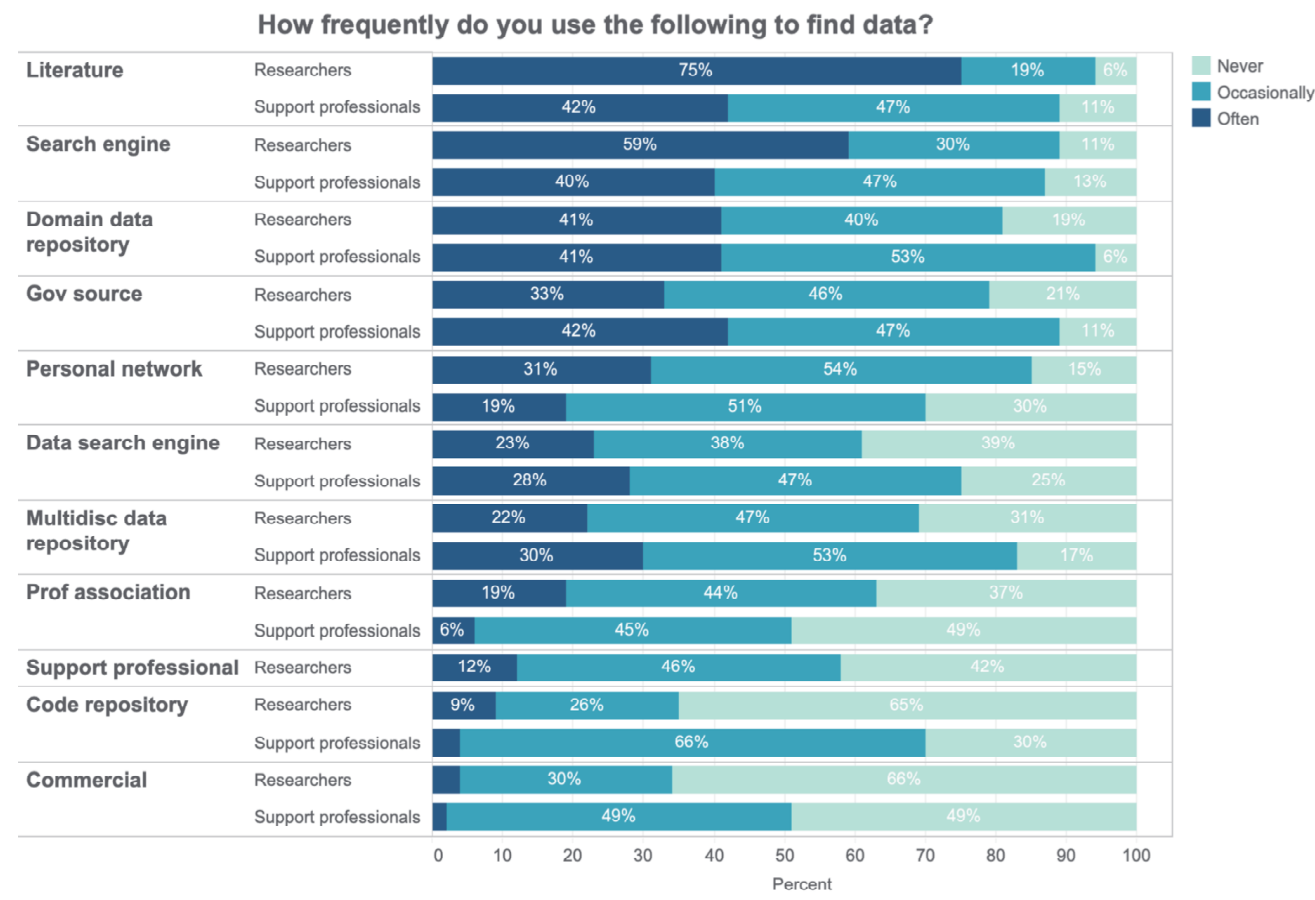

Figure 5.10. Sources used to find data by researchers (including students, managers, and others, $n=$ $1630)$ and research support professionals $(n=47)$. Percent denotes percentage of respondents for each category. Listed in order of decreasing importance for researchers. 
The distribution of sources presented in Figure 5.10 generally holds across disciplines for researchers; literature, followed by search engines or domain repositories, are reported as being used most often in nearly all domains. There are some disciplinary differences, identified by looking at the subset of respondents selecting only one discipline. In the arts and humanities, e.g., turning to research support professionals was selected more often than in other disciplines; in computer science, $76 \%$ of respondents occasionally or often consult code repositories. (A breakdown of the use of sources according to domain for our disciplinary subset is included in the supplementary materials).

Respondents use literature as a source of data - plucking data from reported tables and graphs $^{12}$ - but they also use the literature to track down the original data, making use of behaviors common in literature searching, such as citation chasing (Figure 5.11; the distribution presented in Figure 5.11 remains the same when looking at the percentage of overall responses to this question.) It is common for respondents to first find the literature, and then use the literature as a gateway to locating the data. This strategy is often planned, but it also happens serendipitously while reading or searching for literature. Roughly two thirds of participants also often or occasionally find data serendipitously outside of the literature (e.g. via email or conversations with colleagues) or in the course of sharing or managing data.

Finding data is different because it often occurs as a result of finding academic literature. (Respondent ID 738)

Literature is more direct; data is more like "bonus" finds. One finds interesting data in other contexts of work in a publication, one can contact the author to ask for the data. (Respondent ID 3179)

\footnotetext{
${ }^{12}$ This practice in particular confirms the importance of asking users what they actually do, rather than assuming an ideal notion of data search and reuse.
} 
Which statements describe how you discover data using the academic literature?
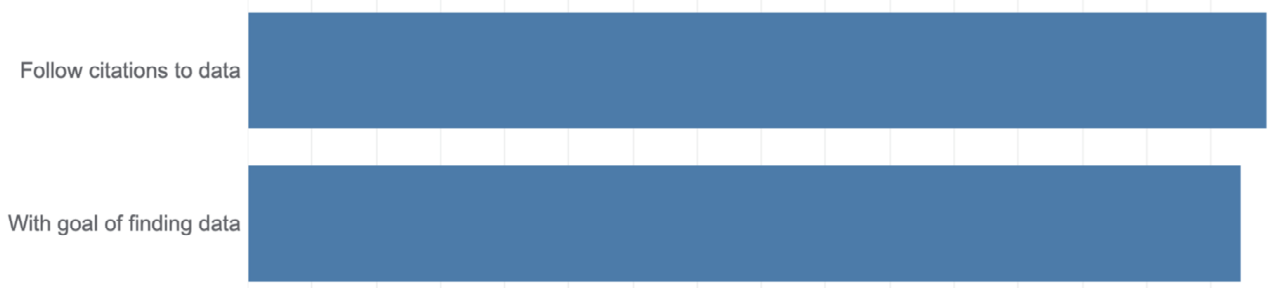

Serendipitously, while reading or searching for literature

Etract data directly from literature, tables or graphs
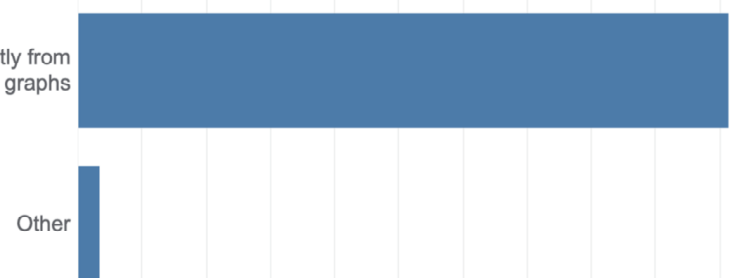

Figure 5.11. Strategies for using the academic literature to discover data. Question asked to respondents who indicated using literature as a source. Percent denotes percentage of respondents; multiple answers were possible $(n=1573)$.

\section{Via social connections}

Using social connections and personal outreach to discover and access data is another important difference identified between literature search and data search. This is reflected in Figure 5.10 where only $15 \%$ of researchers never make use of personal networks in data discovery.

Unlike academic literature where you get the data by accessing the journal, finding data often requires contacting the institution that created the data. (Respondent ID 2357)

I use personal networks and public access datasites to discover data, then I usually have to submit a proposal and get it accepted in order to get access to the data. I have not had the experience of just downloading data directly without going through a permission process. (Respondent ID 1416) 
Attending conferences and having discussions within personal networks are the most frequent ways of mobilizing social connections to discover data (Figure 5.12). While personal networks remain important in actually gaining access to data, contacting data authors directly is the most often reported method for accessing data. Forming new collaborations with data creators also appears to be more important in accessing data than in first discovering them. These patterns hold, regardless of the types of data that respondents need or their intended use for the data, although there are some disciplinary differences in the percentage of respondents discovering or accessing data via conference attendance or forming new collaborations. The need to use personal connections in accessing data also reflects the finding that access remains the largest hurdle for participants.

Do you use the following to discover, access or make sense of data?
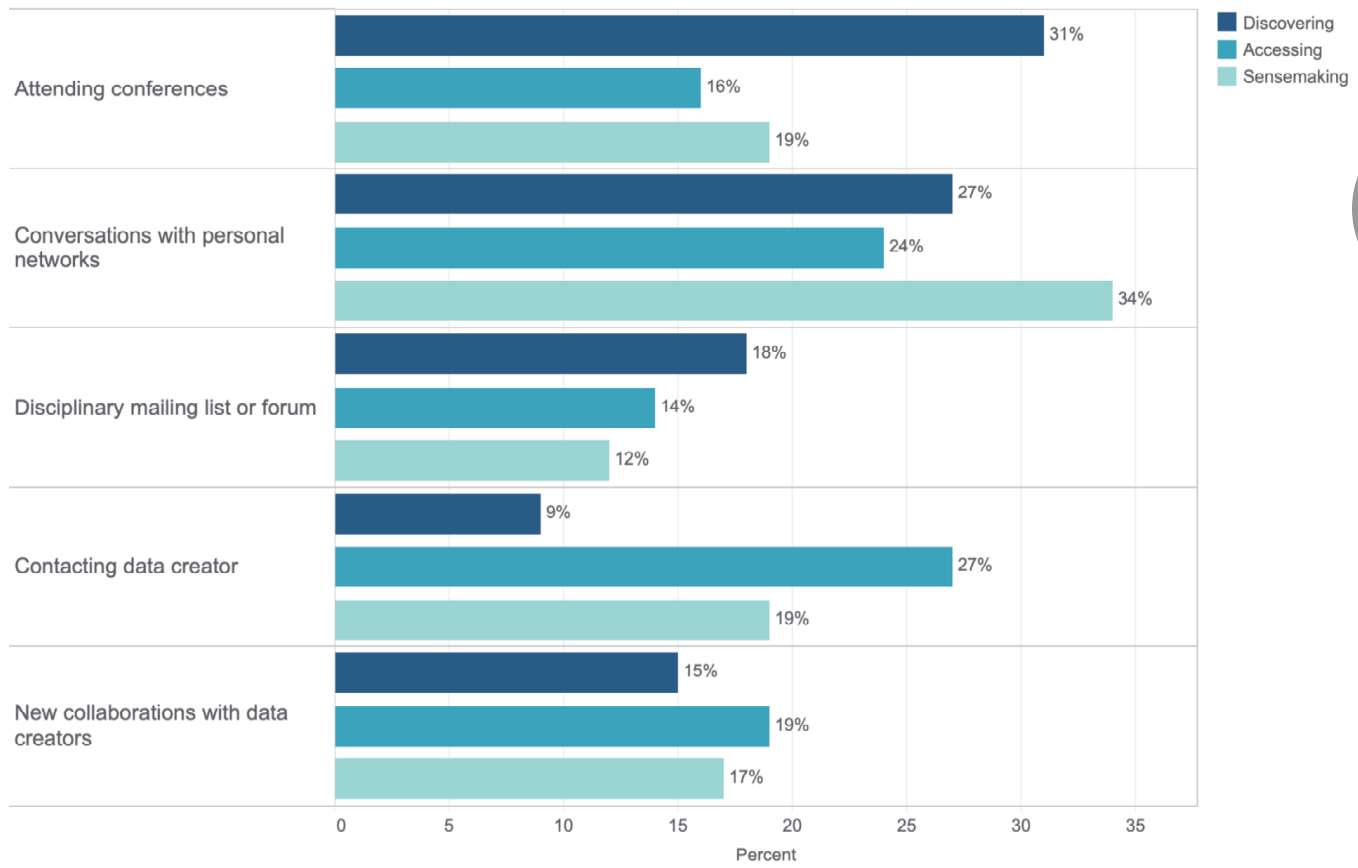

Figure 5.12. How respondents make use of social connections in: discovering data $(n=3311)$, accessing data $(n=3589)$ and making sense of data $(n=3031)$. Percent denotes percentage of responses for each option; multiple responses possible.

Social connections are a two-way street. Not only do respondents seek data from their networks, but experts also receive data without solicitation or in exchange for their knowledge. Participants receive data from individuals both within and outside of their domains of expertise. 
Specialist organizations often seek my help and their members send me data. I write to schools to invite them to participate in the experimental work I do and I analyze their data for them and send them reports and suggestions to overcome the difficulties that I observe. (Respondent ID 1242)

I am an expert in statistics. I didn't have to find the data; the researchers that owned the data find me. I have published a few original articles not being part of my field of expertise as a co-author - as the researcher responsible for the statistical analysis. (Respondent ID 3253)

\section{Via "mediated" search}

For some respondents, actually locating and accessing data is a mediated process, mediated not through the work of information professionals (although this sometimes happens - see Figure 5.10), but rather through the literature and through personal connections. Numerous respondents first discover or encounter data via an "intermediary" source - an article, a conversation with a colleague; they then turn to another source - a data repository, Google - to search specifically for the known data.

I generally do not search for data blindly but I would normally know that it already exists through some previous interaction (reading scientific publication, personal communication). (Respondent ID 679)

General search engines (e.g. Google) can also serve in intermediary roles, as respondents use them not only in order to find data themselves, but also to locate data repositories. These two practices - using Google for known-item searches and to locate repositories rather than data - could contribute to the fact that $38 \%$ of respondents who use general search engines found their searches to be either successful or very successful. However, the majority of respondents using general search engines reported mixed success $(55 \%)$, with $7 \%$ being rarely or never successful, perhaps reflecting the higher failure rate in general in academic search compared to general web searching (Li et al., 2017).

\section{Via specific searches plus casting a wide net}

Much of data searching is very specific. Participants rely on particular, known data repositories and sources. Respondents have specific requirements and search parameters, and seek data for specific purposes and goals, as is evidenced in our typology of data (re)use (Table 5.4). This is in contrast to literature searching, where participants report using 
cross-disciplinary sources, such as the Web of Science or Scopus, and where the goal is often to cast a wide net to discover ideas for use in theory or concept development.

When I search for data I am pretty focused on finding only data sets that I need for a specific purpose. When I search for literature I read papers that are only peripherally related to the subject but they help me formulate new ideas. (Respondent ID 2128)

I tend to search for data by specifying parameters e.g. geographical and date coverage, or by looking for data created by a specific organization. My literature searches are more general and don't have so many search filters applied. (Respondent ID 3688)

In contrast, searching for data is haphazard and less systematic than literature search for many respondents, requiring researchers to cast a wide net to discover distributed data.

It [data search] is a little more haphazard, as I am not as comfortable with finding data. Some of this stems from my not knowing "the" sources, but some of it is also because the finding tools are not yet available. Many times, it is a "try and see" approach. (Respondent ID 3803)

With scientific literature I know for sure where to look for it, before I start the search. In other words, sources are known to me and do not change for years. With data it is always not so. I may find data in unexpected places. (Respondent ID 3152)

\section{By building new practices}

This state of development causes search practices to be in flux, as individuals figure out how best to find and access the data that they need. In contrast to their practices for searching literature, their data search practices are still in formation.

Finding academic literature is part of everyday practice. The processes for finding literature are well-established and institutionally-supported. If I need to find data I have to establish my own process to locate where they are held, get permission from owners, agree access rights etc. (Respondent ID 696) 


\subsubsection{RQ 4: How are people evaluating and making sense of data for (re)use?}

\section{By using varied evaluation criteria and sensemaking strategies}

Respondents require a variety of information about the data and make use of different sensemaking strategies (Figure 5.13). Eighty-nine percent of respondents reported that information about data collection conditions and methodology was important or extremely important in their decisions; information about data processing/handling as well as topical relevance were also ranked highly (Figure 5.13a). The ease (or difficulty) of accessing data is also very important to $73 \%$ of participants. While respondents take the reputation of the data creator into consideration, with $62 \%$ of respondents indicating this is important or extremely important, the reputation of the source of the data (e.g. the repository or journal) appears to be slightly more important, as $71 \%$ of respondents identified the reputation of the source as being important or extremely important. This is further evidenced in Figure 5.16a; $61 \%$ of respondents selected the data creator's reputation as being important/extremely important in establishing trust in secondary data, as compared to $81 \%$ who identified the reputation of the source as being key to developing trust.

How important is the following information when deciding whether to use secondary data?

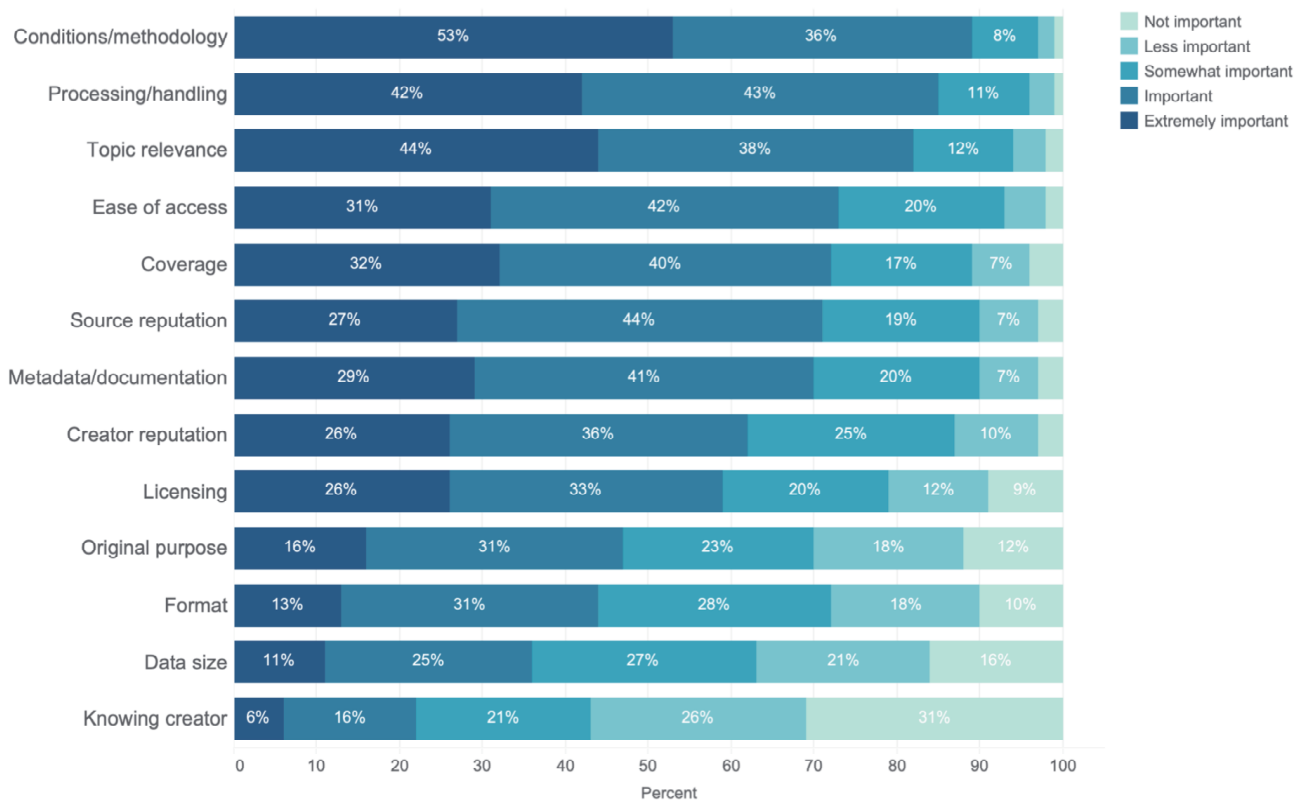

Figure 5.13a) Information used in evaluating data for reuse $(n=1677)$. Percent denotes percentage of respondents. 
How important are the following strategies when deciding to use secondary data?
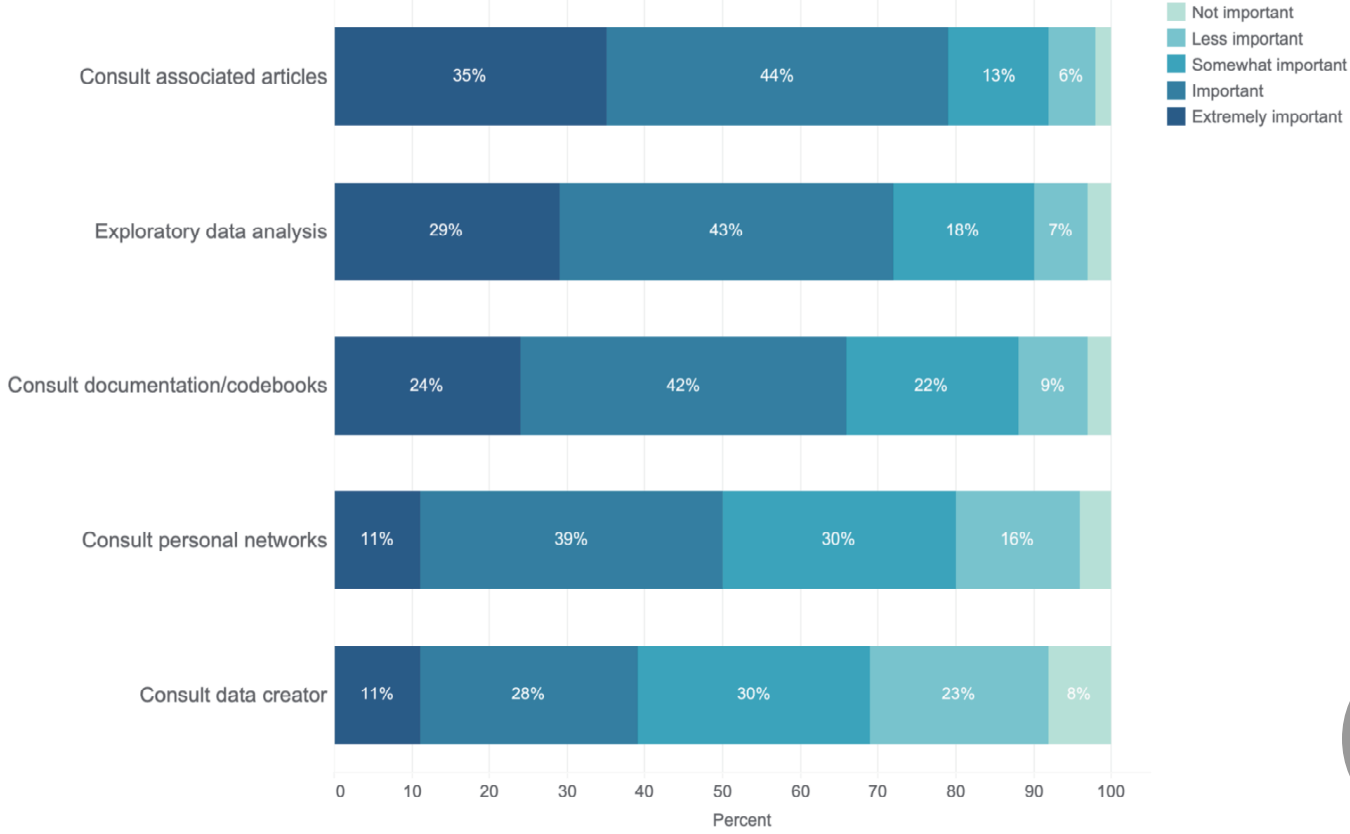

Figure 5.13b) Sensemaking strategies ( $n=1677)$. Percent denotes percentage of respondents.

Other information identified in open responses includes the timeliness of data, prior usage, and the cost of obtaining data, which can determine the type of research that is pursued.

Patent data is free to access. Data on company deals and revenue can sometimes be paid. That requires seeking research funding and typically delays the process. Using publicly free data is quicker. (Respondent ID 3220)

The academic literature plays a key role not only in discovering data, but also in understanding them. Respondents consult associated articles, as well as data documentation and codebooks (Figure 5.11b). Nearly three-quarters of respondents report engaging in exploratory data analysis, i.e. statistical checks or graphical analysis.

Participants also report triangulating data from multiple sources as a way of understanding and determining the validity of data (e.g. Respondent IDs 3131, 2444, 1949).

\section{By using social connections in sensemaking}

Fifty percent of participants reported conversations with personal networks as being key to making sense of data. Conversations with networks are used more often in sensemaking 
than in either discovering or accessing data (Figure 5.12). Contacting data creators to make sense of data does not happen as frequently as discussions with personal networks.

Respondents also attend conferences and form new collaborations to make sense of data (see Figure 5.12). Some variations in the pattern in Figure 5.12 for sensemaking exist across disciplinary domains (e.g. see Figure 5.14), although engaging in conversations with personal networks is almost always chosen most frequently. These variations are likely the result of different disciplinary norms and infrastructures, which influence patterns of collaboration and communication (e.g. the role of conference attendance and publishing norms, or the existence of disciplinary mailing lists and forums, i.e. in computer science).

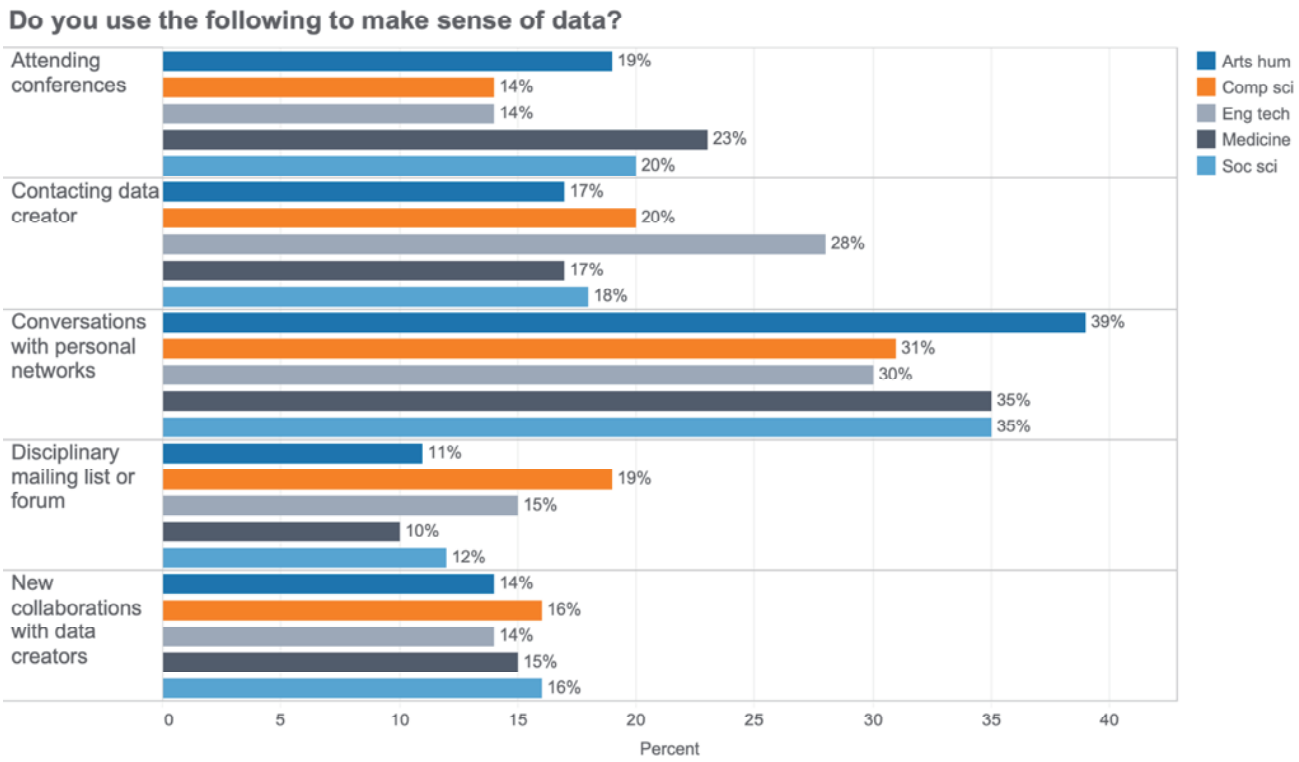

Figure 5.14. Social strategies of sensemaking in five disciplines from the disciplinary subset: arts and humanities ( $n=64)$, computer science $(n=94)$, engineering and technology $(n=109)$, medicine $(n=158)$ and social science $(n=155)$. Percent denotes percentage of responses; multiple responses were allowed.

\section{By using different contextual information for different purposes}

Different data uses are associated with needing different information about data. Figure 5.15 presents significant associations, detected using the statistical test for multiple marginal independence described in the Methodology section, between data uses and evaluation criteria. An association detected with this method indicates that responses to the survey question about evaluation criteria are correlated with responses to the question about data 
uses. In Figure 5.15, we classify the evaluation criteria presented in Figure 5.13a into content-related information (e.g. data collection methods and conditions, the relevance of data to a topic, the exact coverage of the data), structure-related information (format, size, the existence of detailed documentation and metadata), access-related information (ease of access, licensing) and social information (reputation of data creator and source, knowing the data creator). We then plot significant associations which exist between uses in the (re)use typology and these evaluation criteria.

This analysis allows us to begin to identify the types of information needed by respondents in different research phases. It also allows us to identify gaps. Most of the detected associations occur between content-related information and data uses in the project creation/preparation or analysis/sensemaking stages of our (re)use typology. The fewest number of significant associations were detected with calibration and benchmarking. Only one association was found for structure-related information, between teaching and data format; source reputation was also correlated with format and idea generation. The importance of information about how data are processed and handled span uses in all of the research phases; having access to detailed and complete metadata or documentation was found to be associated with experimenting with new techniques and methods, with integration, and with making comparisons. 


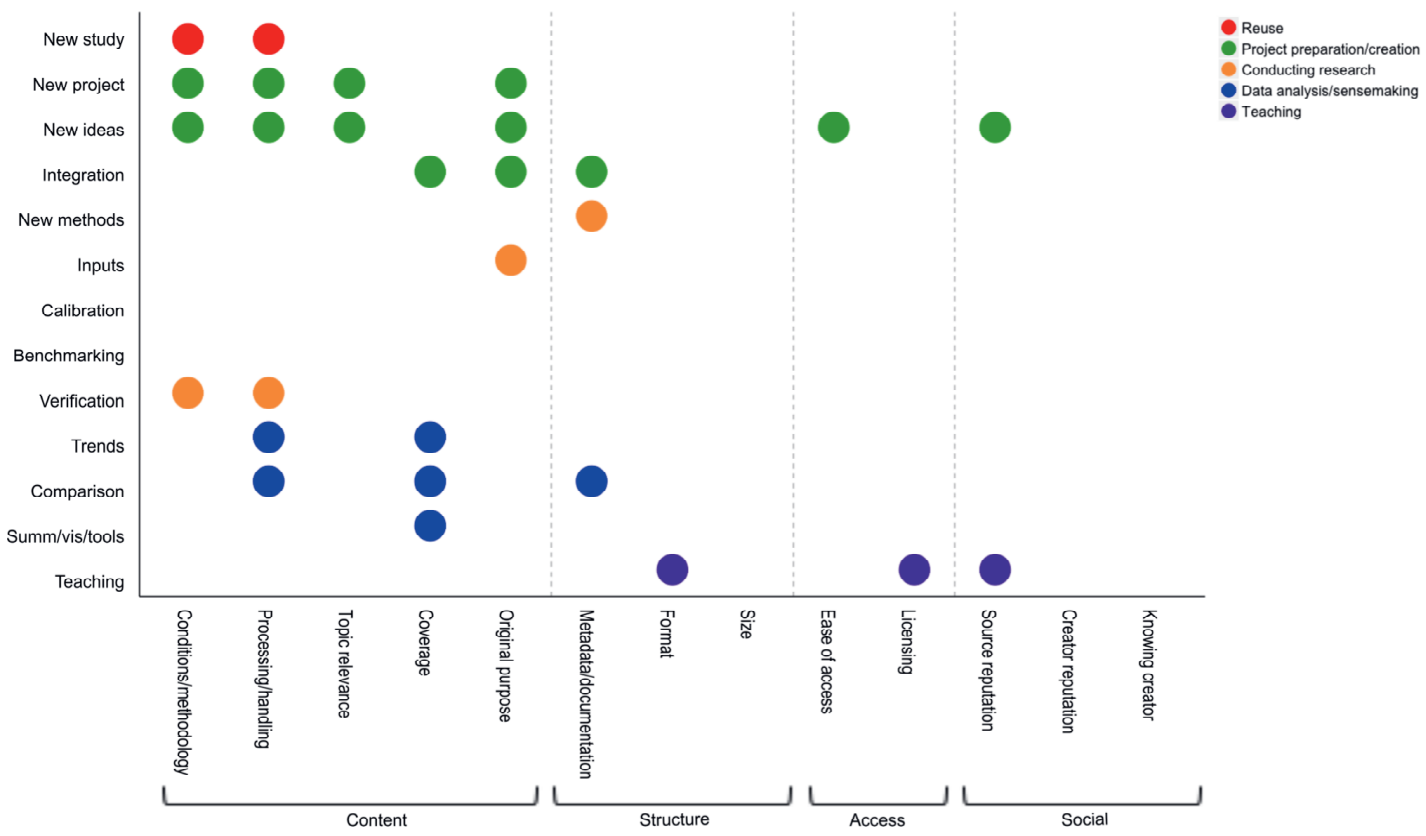

Figure 5.15. Significant associations between types of data use and information important in evaluating data. Associations detected using adjusted Bonferroni test for multiple marginal independence (significance level: $p<0.05$ ). Colors represent phases of (re)use typology. Classifications of evaluation criteria marked with brackets.

\section{By establishing trust and data quality}

The transparency of data collection methods, followed by the reputation of the source and a minimum of errors are critical in trust development (Figure 5.16a). 
What is important in helping to establish trust in secondary data?

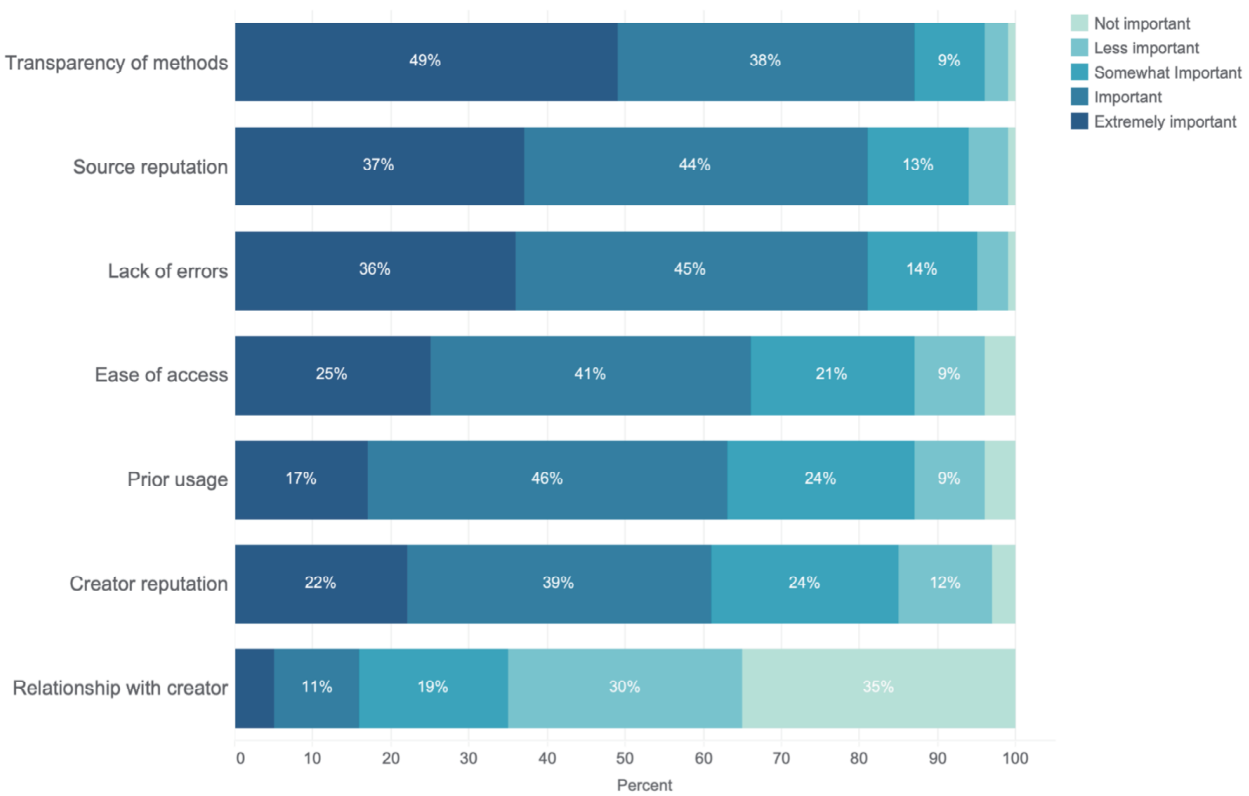

Figure 5.16a). Importance of criteria used to establish trust in secondary data $(n=1677)$. Percent denotes percentage of respondents.

What is important in helping to establish the quality of secondary data?
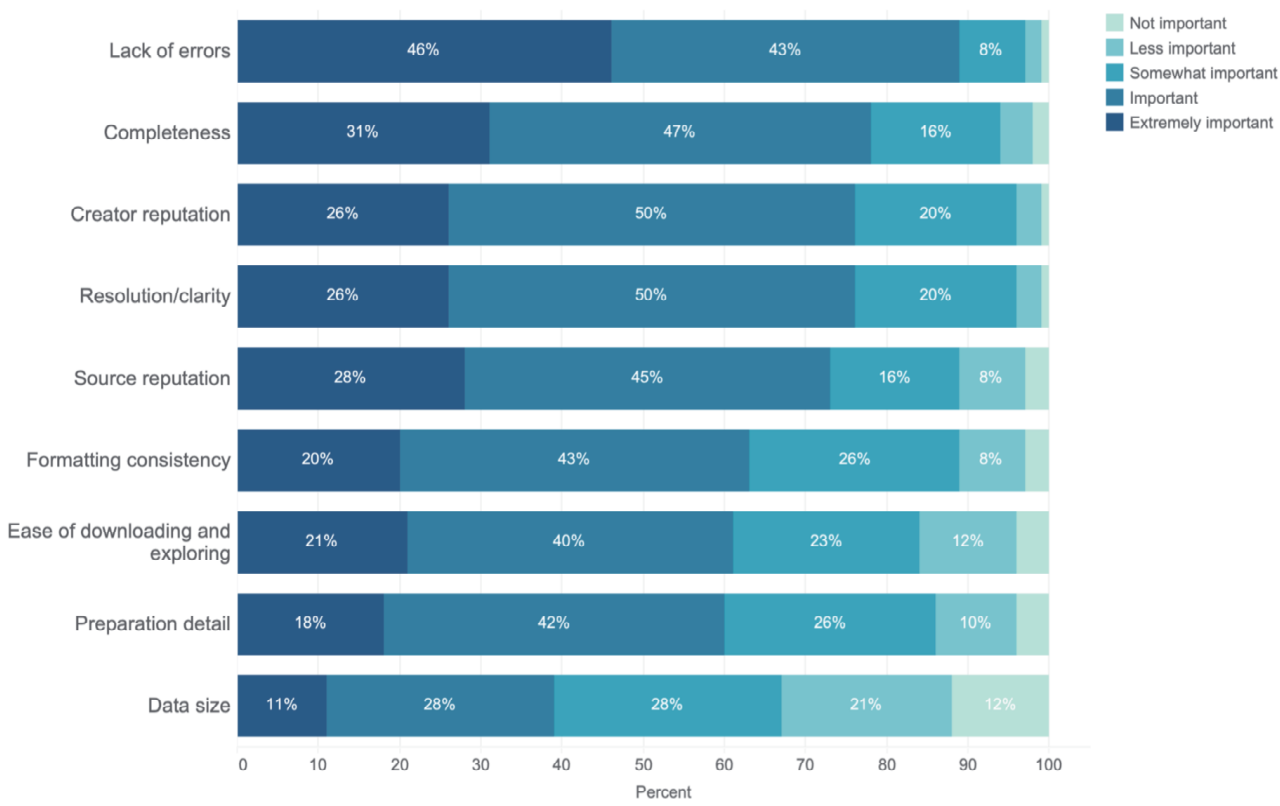

Figure 5.16b) Importance of criteria used to establish quality of secondary data ( $n=1677)$. Percent denotes percentage of respondents. 
In some disciplines, a completely error-free dataset may actually raise suspicions, as it may indicate that the data have been tampered with or manipulated.

Lack of errors would not necessarily help establish trust - errors are normal, so a perfect dataset without errors might be a fabricated dataset. (Respondent ID 3970)

If you know the field you also know what to look for with respect to unreliable data. Sometimes the occasional error actually speaks to the reliability of a dataset: It indicates a person was involved somewhere in data entry. (Respondent ID 1648)

Establishing data quality also depends heavily on the absence of errors and data completeness. Both developing trust and determining quality involve social considerations (Yoon, 2017b, Faniel \& Yakel, 2017). Although respondents across disciplines consistently ranked having a personal relationship with the data creator as being unimportant in establishing trust, they still weigh other "social" factors - i.e. thinking about human involvement in data creation or the reputation of the source - in their decisions. The reputation of the data creator appears to be more important to respondents when evaluating data quality than in trust development.

\subsection{Discussion}

We identify and apply four analytical themes to further discuss our findings about practices of data seeking and reuse. We also consider each theme's relation to recent efforts to increase the discoverability of research data before concluding by suggesting future areas for both practical and conceptual work.

\subsubsection{Communities of use}

The term "community" is often used without considering how communities are formed or their exact composition. Community boundaries are shifting and porous, rather than fixed and stable, and individuals often belong to multiple communities simultaneously (Birnholtz \& Bietz, 2003).

We see this clearly in our data. Although communities are typically thought of in terms of disciplinary domains, more than half of our respondents identified with multiple disciplines. Fifty percent also indicated needing data outside of their domains of expertise, perhaps reflecting funders' efforts to promote interdisciplinary research (Allmendinger, 2015). Data communities can also be thought of in terms of the type of data that a particular group uses 
(Cooper \& Springer, 2019; Gregory, Groth, et al., 2019). However, we show here that respondents need multiple types of data for their work, and that these data needs can be difficult to classify in broad terms.

Communities can form around particular methodologies and ways of using and working with data (Leonelli \& Ankeny, 2015), as is the case, e.g. in the digital humanities and sociology or economics (Levallois et al.,, 2013). Our (re)use typology (Table 5.4) allows for conceptualizing data communities in terms of broad uses of data, e.g. using data in conducting research, and in more specific terms, e.g. using data for calibration. We also found initial signals that particular data uses are associated with needing certain information about data (Figure 5.15). Content-related metadata, such as information about collection conditions and methodologies, is important for our respondents in preparing for new projects; structure-related metadata, e.g. format, is important in teaching. We saw a similar relationship, particularly for teaching data science, in our qualitative data. This suggests another way of conceptualizing data seeking communities - by broad research approaches. Individuals relying on data science techniques, no matter their discipline, may rely more on structure-related information when evaluating data for reuse; content-specific considerations could be more important for more traditional research approaches.

Open data policies and guidelines recognize the importance of communities, but often equate communities with disciplinary domains. The FAIR data principles, e.g. (Wilkinson et al., 2016), call for the use of domain-relevant community standards as well as relevant attributes to facilitate findability and reuse. Our analysis encourages a multi-dimensional way of thinking about communities, recognizing that community-relevant metadata can be defined by considering other factors (e.g. data use) in conjunction with disciplinary domains.

\subsubsection{Interwoven practices}

Data discovery is interwoven with other (re)search practices, particularly searching for academic literature. Roughly eighty percent of respondents stated that their practices for finding data and literature are either sometimes or always the same. The academic literature itself is the go-to source for finding data for the majority of participants. Despite the immature state of data citation practices in many disciplines (Robinson-Garcia et al., 2016), respondents use a strategy common in literature searching - following citations - to locate data from the literature. 
Data citation is not equivalent to bibliographic citation (Borgman, 2016). Most data citations indicate some type of data "usage" (Park \& Wolfram, 2017), but little is known about why people cite data or the details of how data have been used in a work (Silvello, 2018). This presents an additional challenge for people seeking data to use for a particular purpose. A citation model that typifies how data have been used could potentially facilitate data discovery and evaluation practices that begin by following data citations, as well as add value to the multiplicity of data uses that we observed.

\subsubsection{Social connections}

Discovering and accessing data are also mediated by personal networks. Respondents find out about data from their connections and then hunt the data down digitally. This process also occurs in reverse - respondents find data digitally and then access the data by personally contacting data creators. The use of social connections in discovery and sensemaking is intertwined with discipline-specific practices of communication (e.g. the role of conference attendance) and collaborations.

Participants identify using social connections as an important difference between searching and accessing data, as opposed to literature. This difference could be a result of the fact that infrastructures to support data search and access are still in development. It could also be due to the complexity of the sociotechnical issues surrounding data access. Researchers question which data to make available for whom (Levin and Leonelli, 2017), and sensitive data containing private information about participants cannot be made openly accessible.

Accessible data, as defined by the FAIR principles, do not necessarily need to be open data (Mons, et al., 2017). Access to data can be mediated by automated authorization protocols (Wilkinson et al., 2016), but automatic denials of access may not mean that data are completely closed to a human data seeker. Researchers can still contact data authors directly if access is denied to learn more about restrictions and possibly form collaborations that would enable reuse (Gregory et al., 2018). Our results also show that ease of access is a top consideration in using data, especially during early phases of research (Figure 5.15). As certain data become easier to seamlessly and automatically access, other data, those that are more challenging to access, will likely not be used as often, which will shape the research that is or is not pursued. 


\subsubsection{Practices in flux}

Practices and infrastructures are closely linked (Shove et al., 2015); this is especially true for practices of data discovery and reuse. We see this in the tension that we found between specific and haphazard search practices. For some respondents, data infrastructures are still in a state of development, which requires casting a wider net to locate appropriate sources. For others, finding data involves going directly to a particular, well-known data repository in the field.

Data infrastructures consist of assemblages of policies, people, technology and data (Borgman, Darch, et al., 2015, Edwards, 2010). As data are described in more standardized ways, repositories will have different methods of structuring data, be linked to other data and repositories more seamlessly, and will build new services. These services and linkages will change how data seekers interact with and discover data.

Innovations combining new technologies with existing practices will not only alter current practices, but will also bring new considerations to the forefront. Executable papers (e.g. Gil et al., 2016) where readers can interact with data directly, build on linkages between literature and data searching as well as the importance of exploratory data analysis in sensemaking. They also blur the line between where a paper ends and data begins. As the boundaries between data and papers become less defined, the importance of archiving those data in sustainable ways (e.g. Vander Sande et al., 2018) and questions of properly citing data creators, rather than paper authors, will become more visible.

\subsection{Conclusion}

In summary, we have examined the data needs, uses, discovery strategies and sensemaking practices for the 1677 respondents to our survey by presenting an initial quantitative analysis and by drawing on the qualitative survey data. Possible practical applications for this work are many. We conclude by highlighting some key takeaways from our analysis and draw attention to their potential applications, in particular for designers of data discovery systems and managers of data repositories.

\subsubsection{Diversity is normal, not abnormal}

Past in-depth ethnographic work has documented the diversity of data practices in particular research groups and projects within disciplines such as astronomy, environmental engineering and biomedicine (see Borgman, Wofford, et al., 2019). Both our quantitative and 
qualitative results show that this multiplicity is not limited to these specific communities. Rather than being the exception, a diversity of data needs, data uses and data sources appears to be the rule. We also see signals of other forms of diversity in our results, finding, e.g. that data needs and search practices are both specific and broad, that data uses are spread across phases of research and that practices of data discovery are intermingled with other search practices.

We have suggested elsewhere that data discovery systems implement a variety of differentiated search interfaces, including visual and graphical navigation systems, for different users (Gregory, Cousijn, et al., 2019), which would allow data seekers freedom to explore data in different ways. The diversity we observe here also supports the creation of flexible, interlinked designs for data discovery systems and repositories with different levels of specificity. Linking diverse data across locations via standardized approaches is gaining significant momentum (see, e.g. Wilkinson et al., 2016), and would pave the way for general data search engines and federated search efforts. Domain agnostic data search engines (i.e. Google Dataset ${ }^{13}$ or DataSearch ${ }^{14}$ ) allow data seekers to search for data broadly before being directed to data repositories, where more specific searching and exploration is often possible.

This puts an onus on repositories, however, as they become responsible for implementing a new generation of tools supporting more specific search and sensemaking activities within their environments (i.e. generous interfaces (Whitelaw 2015; Mutschke et al. 2014)).

Data search engines could also feasibly help searchers looking for data outside of their domain to better identify potential repositories of interest. Our findings also suggest the need to integrate data search tools with literature databases and data management tools, which we also suggested based on our earlier interviews with data seekers (Gregory, Cousijn, et al., 2019). Figure 5.9 shows that this integration may be more relevant for certain disciplines than for others.

\subsubsection{Communities of use as an entry point to design}

While we suggested differentiated interfaces for different users in the past, the multiplicity of practices we observed makes it challenging to design tools based on "user profiles;" there is also often a gap between the user profiles which designers imagine and the actual users themselves (see Wyatt, 2008). Both our quantitative and qualitative data suggest that

\footnotetext{
${ }^{13} \mathrm{https} / / /$ toolbox.google.com/datasetsearch

${ }^{14} \mathrm{https}: / /$ datasearch.elsevier.com
} 
searching for data is purpose-driven; researchers look for data for a specific purpose or use. This finding is supported by Koesten et al., who use interviews with data professionals and log analyses to suggest that data search is task-dependent (2017). We suggest here also that data communities can be conceptualized by uses of data and show in Figure 5.7 that these uses, although spread across research phases and not limited to a particular point in time (see also Pasquetto et al., 2019), appear to be limited to a core set. This set of data uses could provide an entry point for design, allowing data seekers to search for or filter by data used for these specific purposes.

The set of uses we identify is general and needs further research to test its comprehensiveness. Performing cluster analyses using the data from our survey may be one way to further identify and validate the communities of use which we propose. It is also likely that repositories may be able to identify their own specific communities of use. Identifying these will require innovative approaches by repositories and communication with both data depositors and consumers (see Section 5.6.4).

\subsubsection{Metadata to support sensemaking and reuse}

Metadata plays a key role in facilitating data reuse (Pasquetto et al., 2019; Mayernik, 2016; Mayernik \& Acker, 2018); however, the general metadata needed for discovery, is often not rich enough to support the sensemaking needed for reuse (Zimmerman, 2007). In Figure 5.13 and 5.16 , we identify common evaluation criteria and considerations in developing trust and quality which could inform the development of metadata, at both broader and more specific levels, to support making decisions about using data. Figure 5.15, in particular suggests that certain evaluation criteria can be used to support certain types of data uses, although additional testing needs to be done to validate the associations we detected and to investigate how the factors identified in Figure 5.13 and 5.16 vary by use or disciplinary domain.

\subsubsection{Tenacity and value of the social}

An increasing amount of quantitative (e.g. Digital Science et al., 2019) and qualitative work (e.g. Yoon, 2017b) shows that using secondary data requires communication and collaboration. Our findings demonstrate the importance of social interactions in discovery, access, and sensemaking, in particular showing that researchers rely on conversations with personal networks to make sense of secondary data. We believe that these social interactions will continue to be important in data discovery and reuse, and should be seen as something to support when designing systems and repositories. 
Our earlier suggestions, i.e. ranking datasets by social signals or integrating offline and online interactions around data, still hold promise (see Gregory, Cousijn, et al., 2019). We could also see a role for an expanded metadata schema as a way to open a conversation between data producers and multiple data consumers within the context of the data themselves at the repository level. In such a system, both data producers and data consumers could contribute information about the data to specific fields. Data producers could describe their own use of the data as well as their concerns about what potential reusers need to know about the data before reusing them. Data users could describe how they used or plan to use the data, as well as pose questions about the data to other users or to the producer. Such an implementation would result in a layered metadata record, including general metadata needed for discovery, metadata with common elements supporting sensemaking (e.g. those identified in Figure 5.13), and a layer of interactive, or communication-based metadata. In the future, we envision a co-evolution of metadata schemes driven both by designated communities and repository managers. Such a dynamic way to co-construct metadata schemas and indexing could enable repositories to identify new communities of use.

Our quantitative data provides the opportunity to more deeply probe and test the results we present here. Creating multi-level models to further explore the influence of data uses, data types, or disciplinary domains could be a fruitful next step, as could investigating methods to test the generalizability of our data to broader populations. This future work, in conjunction with the results in this paper, could also inform deeper theoretical work. Designing useful, sustainable tools and services requires considering the interconnections between different practices, infrastructures and communities that we have begun to investigate. Further conceptual work needs to be done to highlight these connections in a way that can be easily communicated and that can practically inform design.

\section{Acknowledgments}

We are very grateful to Ricardo Moreira for his advice and help in organizing, scripting, distributing and managing the survey and to Helena Cousijn for her advice in designing the survey. We would also like to thank Natalie Koziol for her assistance with using the MRCV package. This work is part of the project Re-SEARCH: Contextual Search for Research Data and was funded by the NWO Grant 652.001 .002 


\section{Author Contribution Statement}

KG created the survey, analyzed the data and wrote the paper. PG provided input and feedback on the survey and data analysis as well as drafts of the paper. AS and SW provided input and feedback on the survey design and earlier drafts of the paper.

\subsection{Supplementary material}

\subsubsection{Appendix A: Survey questionnaire}

\section{Introduction}

This study investigates how participants locate and evaluate data they do not create themselves.

The survey consists of three main sections:

- Part 1: Data Needs

- Part 2: Finding Data

- Part 3: Evaluating Data

Our funding comes from the Netherlands Organization for Scientific Research (NWO). The study is part of a collaborative research project between researchers at the Data Archiving and Networked Services (DANS), the University of Amsterdam, the Vrije Universiteit Amsterdam and Elsevier.

By clicking on the below button to start the survey, you indicate your consent to participate in this research. You can read more about the survey and what will be done with the data here (this will launch a new window).

Thank you for your participation.

Please click >> button to indicate consent to participate and to begin the survey. 


\section{Survey Questions}

\section{Part 1: Data Needs}

\section{Q1: Which of the following best describes you?}

Please select one answer

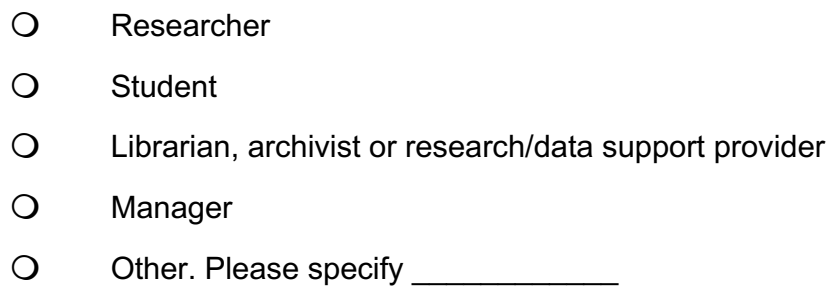

Q2: Please describe the secondary data that you (might) need. (We define secondary data as data that you do not create yourself).

Please write your answer in the box below:

Q3: Please select the options that describe the secondary data that you (might) need. Please select all that apply

- Observational or empirical (e.g. sensor data, survey data, interview transcripts, sample data, neuroimages, ethnographic data, diaries)

$\square \quad$ Experimental (e.g. gene sequences, chromatograms, toroid magnetic field data)

S Simulation (e.g. climate models, economic models)

$\square \quad$ Derived or compiled (e.g. text and data mining, compiled database, 3D models)

$\square \quad$ Other, Please specify

\section{Q4: Why do you use or need secondary data?}

Please select all that apply

As the basis for a new study

- To calibrate instruments or models

$\square \quad$ For benchmarking 
$\square \quad$ To verify my own data

$\square \quad$ As model, algorithm or system inputs

$\square \quad$ To generate new ideas

$\square \quad$ For teaching/training

- To prepare for a new project or proposal

$\square \quad$ To experiment with new methods and techniques (e.g. to develop data science skills)

To identify trends or make predictions

$\square \quad$ To compare multiple datasets to find commonalities or differences

- To create summaries, visualizations, or analysis tools

- To integrate with other data to create a new dataset

$\square \quad$ Other. Please specify

Q5: Have you ever used data outside of your area of expertise?

Please select one answer

O Yes

O No

Q5a: How did you find this data?

Please write your answer in the box below:

\section{Part 2: Finding Data}

\section{Q6: When you need data, who finds it for you?}

Please select all that apply

$\square \quad$ I find it myself

$\square \quad$ Graduate student

$\square \quad$ Research support professional (e.g. librarian, archivist, data or literature manager)

$\square \quad$ Someone else in my personal network (e.g. peers, collaborators, mentors)

$\square \quad$ Other. Please specify 


\section{Q7: How frequently do you use the following to find data?}

Please select one answer per row

\begin{tabular}{l|c|c|c|} 
& Ofte & Occasional & Neve \\
& $\mathrm{n}$ & $\mathrm{ly}$ & $\mathrm{r}$ \\
\hline Multidisciplinary data repositories & $\square$ & $\square$ & $\square$ \\
\hline Discipline-specific data repositories & $\square$ & $\square$ & $\square$ \\
\hline Governmental agencies and websites & $\square$ & $\square$ & $\square$ \\
\hline Personal networks (e.g. colleagues, peers) & $\square$ & $\square$ & $\square$ \\
\hline $\begin{array}{l}\text { Academic literature (e.g. journal articles, conference } \\
\text { proceedings }\end{array}$ & $\square$ & $\square$ & $\square$ \\
\hline Code repository (e.g. GitHub) & $\square$ & $\square$ & $\square$ \\
\hline General search engines (e.g. Google) & $\square$ & $\square$ & $\square$ \\
\hline Professional associations & $\square$ & $\square$ & $\square$ \\
\hline Data specific search engines & $\square$ & $\square$ & $\square$ \\
\hline Commercial sources & & & $\square$
\end{tabular}

Q7_open: Please specify any other resources that you use to find data:

Please write your answer in the box below:

Q7a: Which statement(s) describe how you discover data using the academic literature?

Please select all that apply

I I search the academic literature with the goal of finding data.

$\square \quad$ I find data serendipitously while reading articles or performing literature searches.

I I follow citations and references in the literature to datasets.

$\square \quad$ I extract and use data from the literature directly (e.g. from tables, graphs, or instrument specifications and parameters)

$\square \quad$ Other. Please specify 
Q7b How successful are you at finding data with a general search engine (e.g.

\section{Google)?}

Please select one answer

V Very successful

O Successful

Sometime successful, sometimes not

Rarely successful

O Not successful

Q8: How frequently do you find data in the following ways?

Please select one answer per row

\begin{tabular}{l|c|c|c|} 
& $\begin{array}{c}\text { Ofte } \\
\mathrm{n}\end{array}$ & $\begin{array}{c}\text { Occasional } \\
\text { ly }\end{array}$ & $\begin{array}{c}\text { Neve } \\
\mathrm{r}\end{array}$ \\
\hline By actively searching for data in an online resource & $\square$ & $\square$ & $\square$ \\
\hline $\begin{array}{l}\text { Serendipitously, when searching for something else (e.g. } \\
\text { when looking for journal articles or news) }\end{array}$ & $\square$ & $\square$ & $\square$ \\
\hline $\begin{array}{l}\text { Serendipitously, when NOT actively looking for something } \\
\text { else (e.g. via an email notice or interaction with a colleague) }\end{array}$ & $\square$ & $\square$ & $\square$ \\
\hline In the course of sharing or managing my own data & $\square$ & $\square$ & $\square$
\end{tabular}

Q9: Please indicate if you use the following to discover, access, or make sense of data.

Please select all that apply

\begin{tabular}{l|c|c|c|} 
& $\begin{array}{c}\text { Q10a - } \\
\text { Discover }\end{array}$ & $\begin{array}{c}\text { Q10b - } \\
\text { Access }\end{array}$ & $\begin{array}{c}\text { Q10c - Making } \\
\text { sense of data }\end{array}$ \\
\hline $\begin{array}{l}\text { Conversations with personal networks } \\
\text { (e.g. colleagues, peers) }\end{array}$ & $\square$ & $\square$ & $\square$ \\
\hline Contacting the data creator & $\square$ & $\square$ & $\square$ \\
\hline $\begin{array}{l}\text { Developing new academic collaborations } \\
\text { with data creators }\end{array}$ & $\square$ & $\square$ & $\square$ \\
\hline Attending conferences & $\square$ & $\square$ & $\square$ \\
\hline $\begin{array}{l}\text { Disciplinary mailing lists or discussion } \\
\text { forums }\end{array}$ & $\square$ & $\square$ & $\square$
\end{tabular}


Q10: Do you discover data differently than how you discover academic literature?

Please select one answer

O Yes

O Sometimes

No

Q10a: How is your process for finding data different than your process for finding academic literature?

Please write your answer in the box below:

Q11: How easy is it to find data?

Please select one answer

Easy

$\bigcirc \quad$ Sometimes challenging

O Difficult

Q11a: Why is it challenging to find the data that you need?

Please select all that apply

$\square \quad$ The data are not accessible (e.g. behind paywalls, held by industry).

$\square \quad$ I don't know where or how to best look for the data.

$\square \quad$ The data are located in many different places.

$\square \quad$ The data are not digital.

$\square \quad$ Online search tools are inadequate.

$\square \quad$ I do not have the personal network needed to find or access the data.

$\square \quad$ Other. Please specify 


\section{Part 3: Evaluating Data}

Q12: Please indicate the importance of the following information when deciding whether or not to use secondary data.

Please select one answer per row

\begin{tabular}{|c|c|c|c|c|c|}
\hline & $\begin{array}{l}\text { Extremely } \\
\text { important }\end{array}$ & $\begin{array}{c}\text { Importa } \\
\text { nt }\end{array}$ & $\begin{array}{l}\text { Somewhat } \\
\text { important }\end{array}$ & $\begin{array}{l}\text { Less } \\
\text { important }\end{array}$ & $\begin{array}{l}\text { Not } \\
\text { important }\end{array}$ \\
\hline $\begin{array}{l}\text { Data collection conditions } \\
\text { and methodology }\end{array}$ & $\square$ & $\square$ & $\square$ & $\square$ & $\square$ \\
\hline $\begin{array}{l}\text { How data has been } \\
\text { processed and handled }\end{array}$ & $\square$ & $\square$ & $\square$ & $\square$ & $\square$ \\
\hline Reputation of data creator & $\square$ & $\square$ & $\square$ & $\square$ & $\square$ \\
\hline $\begin{array}{l}\text { Personally knowing the } \\
\text { data creator }\end{array}$ & $\square$ & $\square$ & $\square$ & $\square$ & $\square$ \\
\hline $\begin{array}{l}\text { Reputation of data source } \\
\text { (e.g. repository or journal) }\end{array}$ & $\square$ & $\square$ & $\square$ & $\square$ & $\square$ \\
\hline $\begin{array}{l}\text { Detailed and complete } \\
\text { metadata and } \\
\text { documentation }\end{array}$ & $\square$ & $\square$ & $\square$ & $\square$ & $\square$ \\
\hline Data size & $\square$ & $\square$ & $\square$ & $\square$ & $\square$ \\
\hline Data format & $\square$ & $\square$ & $\square$ & $\square$ & $\square$ \\
\hline $\begin{array}{l}\text { Licensing/copyright } \\
\text { conditions }\end{array}$ & $\square$ & $\square$ & $\square$ & $\square$ & $\square$ \\
\hline $\begin{array}{l}\text { Correct coverage (time, } \\
\text { location, population, etc.) }\end{array}$ & $\square$ & $\square$ & $\square$ & $\square$ & $\square$ \\
\hline $\begin{array}{l}\text { Original purpose of the } \\
\text { data }\end{array}$ & $\square$ & $\square$ & $\square$ & $\square$ & $\square$ \\
\hline Ease of access & $\square$ & $\square$ & $\square$ & $\square$ & $\square$ \\
\hline Topic relevance & $\square$ & $\square$ & $\square$ & $\square$ & $\square$ \\
\hline
\end{tabular}

Q12_open: Please specify any other information you consider when deciding whether to use or not secondary data.

Please write your answer in the box below: 
Q13: How important are the following strategies in evaluating and making sense of data?

Please select one answer per row

\begin{tabular}{l|c|c|c|c|c|} 
& $\begin{array}{c}\text { Extremely } \\
\text { important }\end{array}$ & $\begin{array}{c}\text { Importa } \\
\text { nt }\end{array}$ & $\begin{array}{c}\text { Somewhat } \\
\text { important }\end{array}$ & $\begin{array}{c}\text { Less } \\
\text { important }\end{array}$ & $\begin{array}{c}\text { Not } \\
\text { important }\end{array}$ \\
\hline $\begin{array}{l}\text { Consulting associated } \\
\text { journal articles }\end{array}$ & $\square$ & $\square$ & $\square$ & $\square$ & $\square$ \\
\hline $\begin{array}{l}\text { Consulting data } \\
\text { documentation and }\end{array}$ & $\square$ & $\square$ & $\square$ & $\square$ & $\square$ \\
\begin{tabular}{l} 
codebooks \\
\hline $\begin{array}{l}\text { Consulting the data } \\
\text { creator }\end{array}$
\end{tabular}$\quad \square$ & $\square$ & $\square$ & $\square$ & $\square$ \\
\hline $\begin{array}{l}\text { Consulting personal } \\
\text { networks (e.g. colleagues, } \\
\text { peers) }\end{array}$ & $\square$ & $\square$ & $\square$ & $\square$ & $\square$ \\
\hline $\begin{array}{l}\text { Exploratory data analysis } \\
\text { (e.g. statistical checks, } \\
\text { graphical analysis) }\end{array}$ & $\square$ & $\square$ & $\square$ & $\square$ & $\square$ \\
\hline
\end{tabular}

Q13_open: Please specify any other strategies you consider to evaluate and make sense of data.

Please write your answer in the box below:

Q14: Please indicate the importance of the following in helping you to establish trust in secondary data.

Please select one answer per row

\begin{tabular}{l|c|c|c|c|c|} 
& $\begin{array}{c}\text { Extremely } \\
\text { important }\end{array}$ & $\begin{array}{c}\text { Importa } \\
\text { nt }\end{array}$ & $\begin{array}{c}\text { Somewhat } \\
\text { important }\end{array}$ & $\begin{array}{c}\text { Less } \\
\text { important }\end{array}$ & $\begin{array}{c}\text { Not } \\
\text { important }\end{array}$ \\
\hline $\begin{array}{l}\text { Others' prior usage of } \\
\text { the data }\end{array}$ & $\square$ & $\square$ & $\square$ & $\square$ & $\square$ \\
\hline $\begin{array}{l}\text { Reputation of source } \\
\text { (e.g. repository, } \\
\text { journal) }\end{array}$ & $\square$ & $\square$ & $\square$ & $\square$ & $\square$
\end{tabular}




\begin{tabular}{l|c|c|c|c|c|}
\hline $\begin{array}{l}\text { Reputation of data } \\
\text { creator }\end{array}$ & $\square$ & $\square$ & $\square$ & $\square$ \\
\hline $\begin{array}{l}\text { Transparency in data } \\
\text { collection methods }\end{array}$ & $\square$ & $\square$ & $\square$ & $\square$ & $\square$ \\
\hline Lack of errors & $\square$ & $\square$ & $\square$ & $\square$ & $\square$ \\
\hline Ease of access & $\square$ & $\square$ & $\square$ & $\square$ & $\square$ \\
\hline $\begin{array}{l}\text { Personal relationship } \\
\text { with the data creator }\end{array}$ & $\square$ & $\square$ & $\square$ & $\square$ & $\square$
\end{tabular}

Q14_open: Please specify any other important aspects you consider to help establish trust in secondary data.

Please write your answer in the box below:

Q15: Please indicate the importance of the following in helping you to establish the quality of secondary data.

Please select one answer per row

\begin{tabular}{l|c|c|c|c|c} 
& $\begin{array}{c}\text { Extremely } \\
\text { important }\end{array}$ & $\begin{array}{c}\text { Importa } \\
\text { nt }\end{array}$ & $\begin{array}{c}\text { Somewhat } \\
\text { important }\end{array}$ & $\begin{array}{c}\text { Less } \\
\text { important }\end{array}$ & $\begin{array}{c}\text { Not } \\
\text { important }\end{array}$ \\
\hline Lack of errors & $\square$ & $\square$ & $\square$ & $\square$ & $\square$ \\
\hline $\begin{array}{l}\text { Ease of downloading } \\
\text { and exploring data }\end{array}$ & $\square$ & $\square$ & $\square$ & $\square$ & $\square$ \\
\hline Data size & $\square$ & $\square$ & $\square$ & $\square$ & $\square$ \\
\hline Data completeness & $\square$ & $\square$ & $\square$ & $\square$ & $\square$ \\
\hline $\begin{array}{l}\text { Reputation of source } \\
\text { (e.g. repository, journal) }\end{array}$ & $\square$ & $\square$ & $\square$ & $\square$ & $\square$ \\
\hline Resolution or clarity & $\square$ & $\square$ & $\square$ & $\square$ & $\square$ \\
\hline $\begin{array}{l}\text { Reputation of data } \\
\text { creator }\end{array}$ & $\square$ & $\square$ & $\square$ & $\square$ & $\square$ \\
\hline $\begin{array}{l}\text { Detail or amount of } \\
\text { work done to prepare } \\
\text { data }\end{array}$ & $\square$ & $\square$ & $\square$ & $\square$ & $\square$ \\
\hline $\begin{array}{l}\text { Consistency of } \\
\text { formatting }\end{array}$ & $\square$ & $\square$ & $\square$ & $\square$ & $\square$ \\
\hline
\end{tabular}


Q15_open: Please specify any other important aspects you consider to help establish the quality of secondary data.

Please write your answer in the box below:

Part 4: Demographics

You are nearly at the end of the survey. Below are some questions to help us classify your answers.

D1: In which subject discipline do you specialize?

Please check all that apply.

$\square \quad$ Agriculture

口 Arts and Humanities

- Astronomy

$\square \quad$ Biochemistry, Genetics, and

Molecular Biology

$\square \quad$ Biological Sciences

- Business, Management and

Accounting

$\square \quad$ Chemical Engineering

$\square \quad$ Chemistry

$\square \quad$ Computer Sciences / IT

口 Decision Sciences

$\square \quad$ Dentistry

$\square \quad$ Earth and Planetary Sciences

E Economics, Econometrics and

Finance

$\square \quad$ Energy

$\square \quad$ Engineering and Technology $\square \quad$ Health professions

$\square \quad$ Immunology and Microbiology

․ Materials Science

$\square \quad$ Mathematics

口 Medicine

$\square \quad$ Multidisciplinary

$\square \quad$ Neuroscience

$\square \quad$ Nursing

口 Pharmacology, Toxicology and

Pharmaceutics

$\square \quad$ Physics

口 Psychology

$\square \quad$ Social Science

$\square \quad$ Veterinary

$\square \quad$ Information science

$\square \quad$ Other. Please specify 
Environmental Sciences

D2: How many years of professional experience do you have in your field? Please select one answer
O $\quad 0-5$
O 6-15
O $16-30$
O $31+$ 
D3: In which county do you currently work?

\begin{tabular}{|c|c|c|c|}
\hline$\square$ Afghanistan & 口 Brunei & $\square$ East Timor & 口 Honduras \\
\hline Albania & प Brunei Darussalam & Q Ecuador & Q Hong Kong \\
\hline Algeria & 口 Bulgaria & 口 Egypt & Q Hungary \\
\hline 口 American Samoa & 口 Burkina Faso & EI Salvador & $\square$ Iceland \\
\hline Andorra & $\square$ Burundi & 口 Equatorial Guinea & Q India \\
\hline Angola & 口 Cambodia & Q Eritrea & Q Indonesia \\
\hline Anguilla & 口 Cameroon & Q Estonia & $\begin{array}{l}\text { Iran (Islamic Republic } \\
\text { of) }\end{array}$ \\
\hline Q Antarctica & ¿ Canada & 口 Ethiopia & Q Iraq \\
\hline$\square$ Antigua and Barbuda & 口 Cape Verde & $\begin{array}{l}\text { Q Falkland Islands } \\
\text { (Malvinas) }\end{array}$ & $\square$ Ireland \\
\hline$\square$ Argentina & ૫ Cayman Islands & $\mathrm{QFiji}$ & Q Israel \\
\hline$\square$ Armenia & $\begin{array}{l}\text { Q Central African } \\
\text { Republic }\end{array}$ & u Finland & 口 Italy \\
\hline$\square$ Aruba & Q Chad & Q France & 口 Jamaica \\
\hline Australia & 口 Chile & 口 French Guiana & 口 Japan \\
\hline Austria & 口China & 口 French Polynesia & $\square$ Jordan \\
\hline 口 Azerbaijan & 口 Christmas Island & $\begin{array}{l}\text { Q French Southern } \\
\text { Territories }\end{array}$ & 口 Kazakhstan \\
\hline 口 Bahamas & $\begin{array}{l}\text { Q Cocos (Keeling) } \\
\text { Islands }\end{array}$ & 口 Gambia & 口 Kenya \\
\hline Bahrain & प Colombia & $\square$ Georgia & K Kiribati \\
\hline$\square$ Bangladesh & Q Comoros & Q Germany & North Korea \\
\hline Q Barbados & $\square$ Congo & Ghana & $\square$ Kuwait \\
\hline 口 Belarus & 口 Cook Islands & 口 Gibraltar & Kyrgyzstan \\
\hline 口 Belgium & 口 Costa Rica & $\square$ Greece & $\begin{array}{l}\text { Q Lao People's } \\
\text { Democratic Republic }\end{array}$ \\
\hline ૧ Belize & 口 Cote d'Ivoire & $\square$ Greenland & Q Laos \\
\hline$\square$ Benin & Croatia & 口 Grenada & 口 Latvia \\
\hline Q Bermuda & C Cuba & Q Guadeloupe & 口 Lebanon \\
\hline Q Bhutan & 口 Cyprus & 口 Guam & 口 Lesotho \\
\hline Bolivia & - Czech Republic & 口 Guatemala & 口 Liberia \\
\hline $\begin{array}{l}\text { B Bosnia and } \\
\text { Herzegovina }\end{array}$ & 口 Denmark & 口 Guinea-Bissau & $\begin{array}{l}\text { Q Libyan Arab } \\
\text { Jamahiriya }\end{array}$ \\
\hline Q Botswana & 口 Djibouti & 口 Haiti & 口 Lithuania \\
\hline Q Brazil & Q Dominica & $\begin{array}{l}\text { Q Heard Island and } \\
\text { McDonald Islands }\end{array}$ & Q Luxembourg \\
\hline $\begin{array}{l}\square \text { British Indian Ocean } \\
\text { Territory }\end{array}$ & 口 Dominican Republic & $\begin{array}{l}\text { u Holy See (Vatican } \\
\text { City State) }\end{array}$ & 口 Macau \\
\hline
\end{tabular}




\begin{tabular}{|c|c|c|c|}
\hline Q Madagascar & ૫Oman & ૫ Slovenia & QUSA \\
\hline Malawi & 口 Pakistan & 口 Solomon Islands & u Uzbekistan \\
\hline$\square$ Malaysia & 口 Palau & 口 Somalia & $\square$ Vanuatu \\
\hline Q Maldives & 口 Panama & Q South Africa & 口 Venezuela \\
\hline ૫ Mali & 口 Papua New Guinea & 口 South Korea & 口 Viet Nam \\
\hline$\square$ Malta & 口 Paraguay & 口 Spain & Q Virgin Islands \\
\hline$\checkmark$ Martinique & ૧ Peru & 口 Sri Lanka & ૫ Virgin Islands (US) \\
\hline$\square$ Mauritania & 口Philippines & $\square$ Sudan & ૫ Virgin Islands, British \\
\hline Mauritius & Q Pitcairn & Q Suriname & Wallis and Futuna \\
\hline Mexico & C Poland & प Swaziland & ૫Yemen \\
\hline $\begin{array}{l}\text { Micronesia } \\
\text { (Federated States of) }\end{array}$ & 口 Portugal & Q Sweden & ૫ Zambia \\
\hline Monaco & 口 Puerto Rico & 口 Switzerland & 口 Zimbabwe \\
\hline$\square$ Mongolia & 口Q Qatar & 口 Syrian Arab Republic & $\begin{array}{l}\text { Q Palestinian Territory, } \\
\text { Occupied }\end{array}$ \\
\hline$\square$ Montserrat & 口 Reunion & ૫ Taiwan & Moldova, Republic of \\
\hline Morocco & 口Romania & 口 Tajikistan & Q Marshall Islands \\
\hline 口 Mozambique & QRUSSIA & ૫ Tanzania & $\begin{array}{l}\text { Macedonia, The } \\
\text { Former Yugoslav } \\
\text { Republic of }\end{array}$ \\
\hline Q Myanmar & Q Rwanda & Q Thailand & Q Liechtenstein \\
\hline 口 Namibia & 口 Saint Helena & 口 Togo & 口 Korea, Republic of \\
\hline$\square$ Nauru & $\square$ Saint Kitts and Nevis & $\square$ Tonga & 口 Guyana \\
\hline ૫ Nepal & Saint Lucia & Q Trinidad and Tobago & $\square$ Guinea \\
\hline$\square$ Netherlands & $\begin{array}{l}\text { Saint Vincent and the } \\
\text { Grenadines }\end{array}$ & Q Tunisia & 口 Gabon \\
\hline ૫ Netherlands Antilles & ¿ Samoa & 口 Turkey & ૫ Faroe Islands \\
\hline$\square$ New Caledonia & $\begin{array}{l}\square \text { Sao Tome and } \\
\text { Principe }\end{array}$ & 口 Turkmenistan & ૫Zanzibar \\
\hline$\square$ New Zealand & 口 Saudi Arabia & $\begin{array}{l}\square \text { Turks and Caicos } \\
\text { Islands }\end{array}$ & 口 Tokelau \\
\hline ૫ Nicaragua & 口 Senegal & ૫ Uganda & \\
\hline Q Niger & $\begin{array}{l}\text { Serbia and } \\
\text { Montenegro }\end{array}$ & Q Ukraine & \\
\hline$\checkmark$ Nigeria & $\square$ Seychelles & ૫ United Arab Emirates & \\
\hline$\square$ Niue & 口 Sierra Leone & ૫ United Kingdom & \\
\hline ૫ Norfolk Island & ૫ Singapore & $\begin{array}{l}\text { Q United States Minor } \\
\text { Outlying Islands }\end{array}$ & \\
\hline Norway & 口 Slovakia & Q Uruguay & \\
\hline
\end{tabular}

\section{D4: What type of organization do you work for?}

\section{Please select one answer}

O University or college

○ Research institution

O Government agency

O Corporate

Independent archive or library 
Other. Please specify

D5: Please indicate how the following people feel about sharing their research data.

Please select one answer per row

\begin{tabular}{|c|c|c|c|c|c|c|}
\hline & $\begin{array}{c}\text { Data } \\
\text { sharing is } \\
\text { strongly } \\
\text { encourag } \\
\text { ed }\end{array}$ & $\begin{array}{c}\text { Data } \\
\text { sharing is } \\
\text { somewhat } \\
\text { encourage } \\
\text { d }\end{array}$ & $\begin{array}{c}\text { Data } \\
\text { sharing is } \\
\text { neither } \\
\text { encourage } \\
\text { d nor } \\
\text { discourage } \\
\text { d }\end{array}$ & $\begin{array}{c}\text { Data } \\
\text { sharing is } \\
\text { somewhat } \\
\text { discourage } \\
\text { d }\end{array}$ & $\begin{array}{c}\text { Data } \\
\text { sharing is } \\
\text { strongly } \\
\text { discourage } \\
\text { d }\end{array}$ & $\begin{array}{c}\text { Don't } \\
\text { know/ } \\
\text { Not } \\
\text { applicabl } \\
\text { e }\end{array}$ \\
\hline You & $\square$ & $\square$ & $\square$ & $\square$ & $\square$ & $\square$ \\
\hline $\begin{array}{l}\text { The } \\
\text { people } \\
\text { you work } \\
\text { with } \\
\text { directly }\end{array}$ & $\square$ & $\square$ & $\square$ & $\square$ & $\square$ & $\square$ \\
\hline $\begin{array}{l}\text { Your } \\
\text { disciplinar } \\
\text { y } \\
\text { communit } \\
\text { y }\end{array}$ & $\square$ & $\square$ & $\square$ & $\square$ & $\square$ & $\square$ \\
\hline $\begin{array}{l}\text { Your } \\
\text { institution }\end{array}$ & $\square$ & $\square$ & $\square$ & $\square$ & $\square$ & $\square$ \\
\hline
\end{tabular}

D6: Please indicate how the following people feel about reusing data produced by other people.

Please select one answer per row

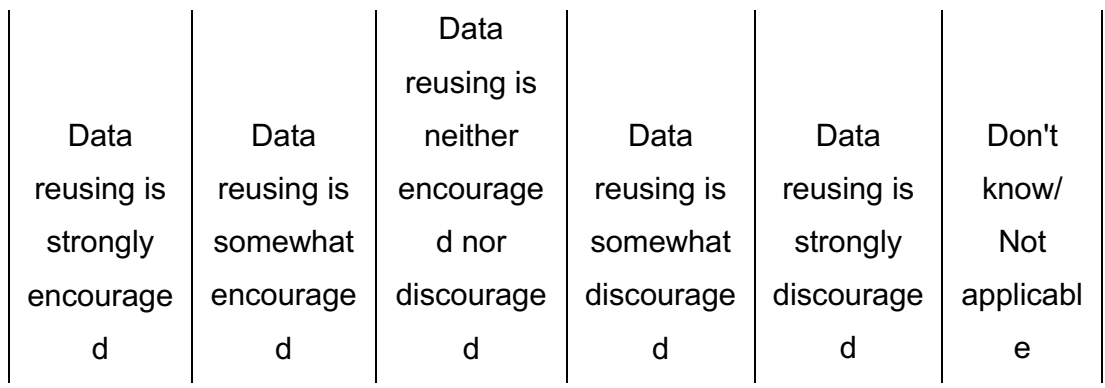




\begin{tabular}{l|c|c|c|c|c|c|}
\hline You & $\square$ & $\square$ & $\square$ & $\square$ & $\square$ & $\square$ \\
\hline The & & & & & & \\
people & & $\square$ & $\square$ & $\square$ & $\square$ & $\square$ \\
you work & $\square$ & & & & \\
with & & & & & \\
directly & & $\square$ & $\square$ & $\square$ & $\square$ & $\square$ \\
\hline $\begin{array}{l}\text { Your } \\
\text { disciplinar }\end{array}$ & & & & & $\square$ & \\
y & & & & & \\
communit & & $\square$ & $\square$ & & $\square$ & \\
y & $\square$ & & & & \\
\hline $\begin{array}{l}\text { Your } \\
\text { institution }\end{array}$ & & & & &
\end{tabular}

D7: Have you ever shared your own research data?

Please select one answer

O Yes

O No

D8: Final comments: Do you have anything else that you would like us to know?

Please write your comments in the box below:

Additional questions asked to participants selecting "Librarian, archivist or research/data support provider" as their role.

L3: Do you use or need secondary data for your own research or to support others?

Please select one answer

For my own research

O To support others

For both my own research and to support others 


\section{L4: Who are the people whom you support?}

Please select all that apply

$\square \quad$ Students

口 Researchers

Industry employees

$\square \quad$ Other. Please specify

\section{L5: How do you support people with their data needs?}

Please select all that apply

$\square \quad$ I teach people about data management planning (e.g. through consultations, workshops, etc.).

$\square \quad$ I teach people how to discover and evaluate data (e.g. through consultations, workshops, etc.).

I I find data for people.

$\square \quad$ I help people to curate their data.

- I find literature for people.

$\square \quad$ Other. Please specify 
5.7.2. Appendix B: P-value tables

\begin{tabular}{|c|c|c|c|c|c|}
\hline & Observ/empirical & Experimental & Deriv/compiled & Simulation & Othe \\
\hline Agricul & 0.647 & $\mathrm{p}<.001^{*}$ & 0.281 & 0.066 & 0 \\
\hline Arts hum & 1.000 & $\mathrm{p}<.001^{*}$ & $p<.001^{*}$ & $\mathrm{p}<.001^{*}$ & $p<.01$ \\
\hline Astronom & 0.103 & 0.92 & 0.008 & $p<.001^{*}$ & 0 \\
\hline Biochem & $p<.001^{*}$ & $\mathrm{p}<.001^{*}$ & 0.92 & 0.003 & 0.2 \\
\hline Biolog & $p<.001^{*}$ & $p<.001^{*}$ & 0.069 & 0.091 & $0 . \varepsilon$ \\
\hline Busin & 0.001 & $p<.001^{*}$ & 0.054 & 0.718 & 0.2 \\
\hline Chem & $p<.001^{*}$ & $p<.001^{*}$ & 0.647 & 0.624 & 0.4 \\
\hline Chem Eng & $p<.001^{*}$ & $p<.001^{*}$ & 1.000 & 0.002 & $0 . \varepsilon$ \\
\hline Comp Sci & 0.92 & 0.032 & $\mathrm{p}<.001^{*}$ & 0.001 & 0.2 \\
\hline Decis Sci & 0.275 & 0.138 & $p<.001$ & 0.001 & 0.7 \\
\hline Dentist & 0.39 & 0.023 & 0.296 & 0.11 & $p<.01$ \\
\hline Earth Plan & 0.003 & 1.000 & 0.02 & $\mathrm{p}<.001^{*}$ & 0 \\
\hline Econ & $p<.001$ & $p<.001^{*}$ & 1,000 & $p<.001^{*}$ & 0.2 \\
\hline Energy & 0.087 & 0.001 & 0.862 & $\mathrm{p}<.001^{\star}$ & 0.6 \\
\hline Eng Tech & 0.009 & 0.022 & 0.187 & $p<.001^{*}$ & 0.4 \\
\hline Environ & 0.098 & 0.841 & 0.647 & $\mathrm{p}<.001^{\star}$ & 0.7 \\
\hline $\begin{array}{l}\text { Health } \\
\text { Prof }\end{array}$ & $p<.001$ & 0.002 & 0.458 & 0.001 & $0 . \varepsilon$ \\
\hline Immun & 0.03 & $p<.001^{*}$ & 0.462 & 0.001 & $0 . E$ \\
\hline Info Sci & 0.002 & 0.001 & $p<.001^{*}$ & 0.031 & $0 . C$ \\
\hline Math & 0.145 & 0.45 & 0.029 & $p<.001^{*}$ & $p<.01$ \\
\hline Matl Sci & $p<.001^{*}$ & $\mathrm{p}<.001^{*}$ & 0.103 & 0.008 & 0 \\
\hline Med & $p<.001^{*}$ & 0.187 & 0.025 & $p<.001^{*}$ & 0.4 \\
\hline Multi & 0.005 & 0.92 & 0.013 & 0.002 & $0 . \varepsilon$ \\
\hline Neuro & 0.137 & 0.123 & 0.764 & 0.09 & 0.6 \\
\hline Nurs & 0.187 & 0.037 & 0.252 & 0.144 & $0 . i$ \\
\hline Pharma & 0.03 & $\mathrm{p}<.001^{*}$ & 0.639 & 0.079 & 0.5 \\
\hline Physics & $p<.001^{*}$ & $p<.001^{\star}$ & 0.806 & $p<.001^{*}$ & $0 . C$ \\
\hline Psych & $\mathrm{p}<.001^{*}$ & 0.006 & 0.458 & 0.001 & $0 . \varepsilon$ \\
\hline Soc Sci & $p<.001^{*}$ & $p<.001^{*}$ & 0.332 & 0.005 & $0 . \overline{7}$ \\
\hline Vet & 0.729 & $p<.001^{*}$ & 0.092 & 0.046 & 0 \\
\hline Other & 0.071 & 0.037 & 0.377 & 0.074 & $0 . c$ \\
\hline
\end{tabular}

Table 5.5. P-value table for Figure 5.6: associations between disciplinary domain and needed data. Significance was determined at the $p<.05$ level with a Bonferroni correction with $m=155$. $P$-values are reported before correction. Significant associations are marked with an asterisk and colored in blue. 


\begin{tabular}{|c|c|c|c|}
\hline & Observ/empirical & Experimental & Deriv/compiled \\
\hline New study & $p<.001^{*}$ & 0.044 & 0.007 \\
\hline New projects & 0.001 & 0.043 & 0.09 \\
\hline New ideas & 0.124 & 0.005 & 0.017 \\
\hline Integration & $\mathrm{p}<.001^{\star}$ & 0.512 & $\mathrm{p}<.001^{\star}$ \\
\hline New methods & 0.33 & $\mathrm{p}<.001^{*}$ & $p<.001^{*}$ \\
\hline Inputs & 0.135 & $p<.001$ & $\mathrm{p}<.001^{*}$ \\
\hline Calibration & 0.242 & $p<.001^{*}$ & $\mathrm{p}<.001^{\star}$ \\
\hline Benchmark & 0.017 & 0.095 & $p<.001^{*}$ \\
\hline Verification & 0.251 & $\mathrm{p}<.001^{*}$ & $\mathrm{p}<.001^{*}$ \\
\hline Trends & $\mathrm{p}<.001^{*}$ & 0.888 & $\mathrm{p}<.001^{*}$ \\
\hline Comparison & $\mathrm{p}<.001^{*}$ & $p<.001^{*}$ & $\mathrm{p}<.001^{*}$ \\
\hline Summ/vis/tools & $p<.001^{*}$ & 0.292 & $p<.001^{*}$ \\
\hline Teaching & $p<.001^{*}$ & 0.624 & 0.151 \\
\hline Other & 0.003 & 0.095 & 0.101 \\
\hline
\end{tabular}

Table 5.6. P-value table for Table 5.4: associations between types of data use and needed data type. Significance was determined at the $p<.05$ level with a Bonferroni correction with $m=70$. P-values are reported before correction. Significant associations are marked with an asterisk and colored in blue. "Other" options are not shown as there were no significant associations present. 

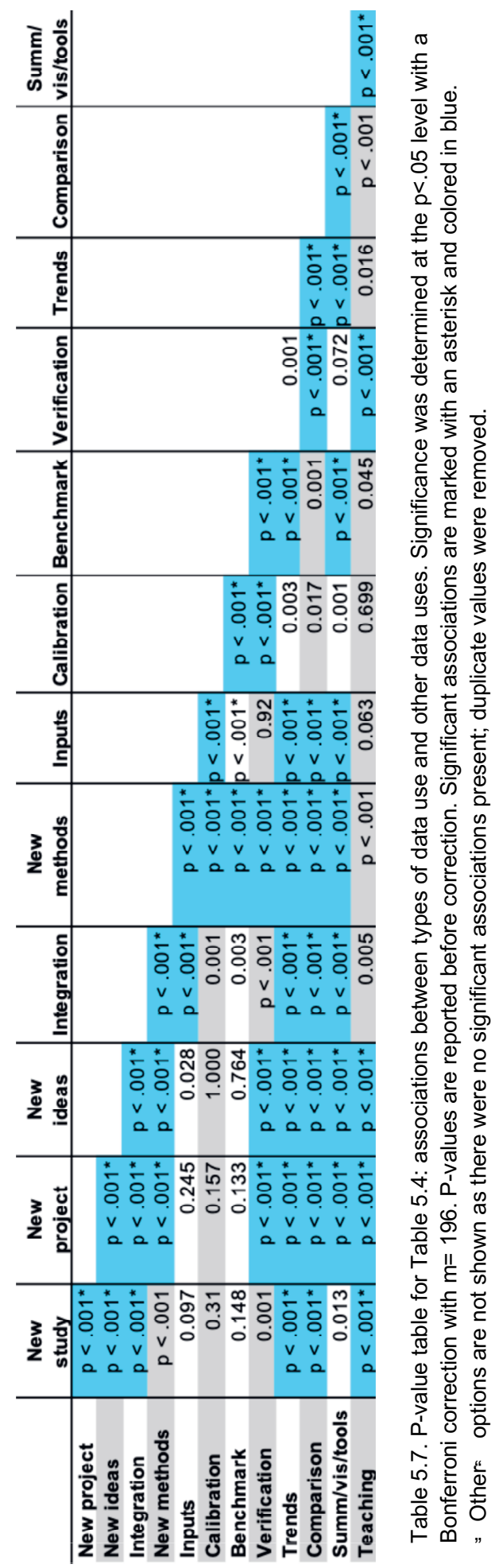


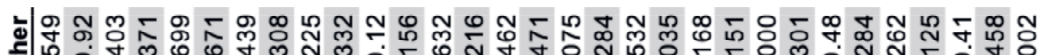

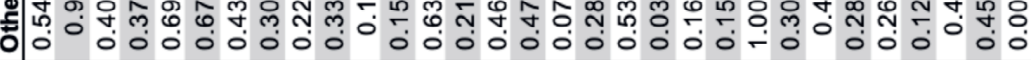

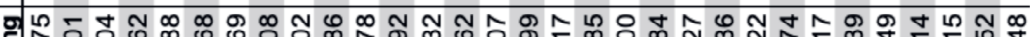

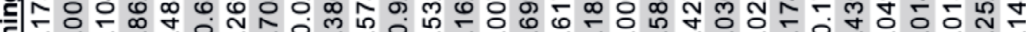

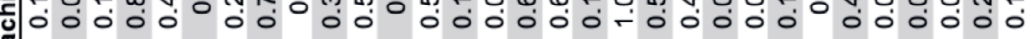

ॄ

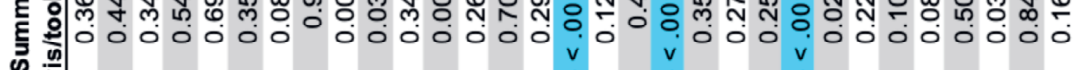

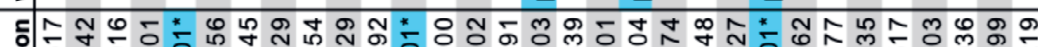

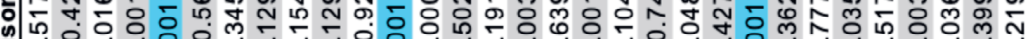

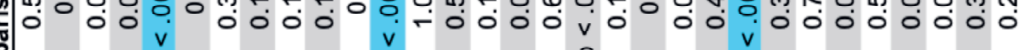

m

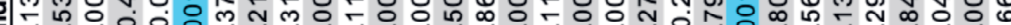

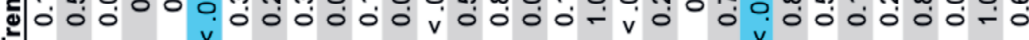

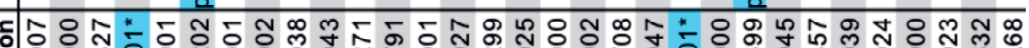

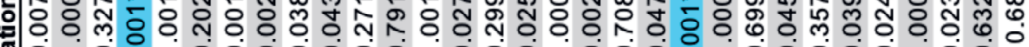

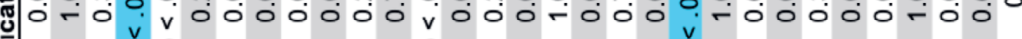

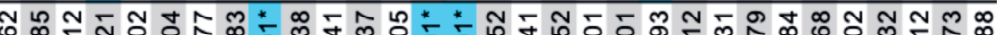
œ =00 $00000^{0} 00$ v 000000000000000

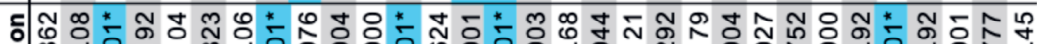

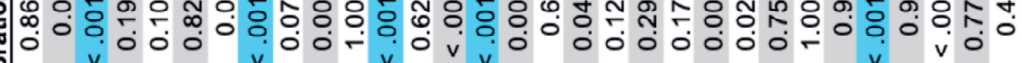

t

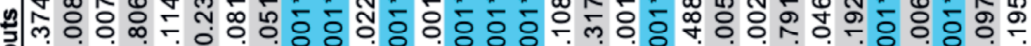

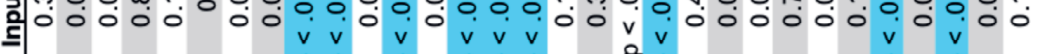

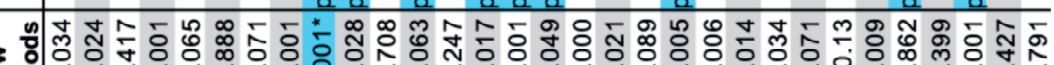

z ह 2 a

โปน์

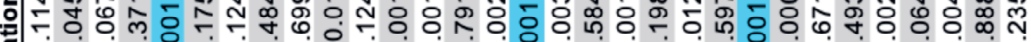

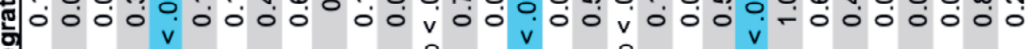
竎 z z 음 a a a

ว

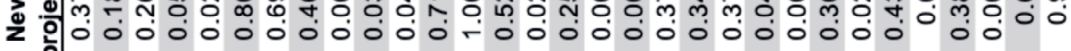

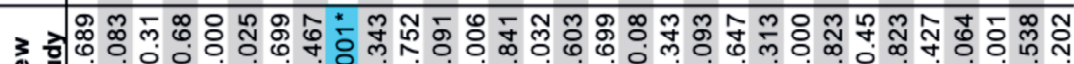

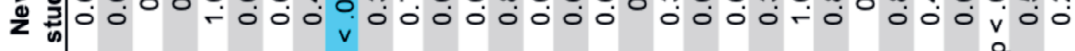
| 


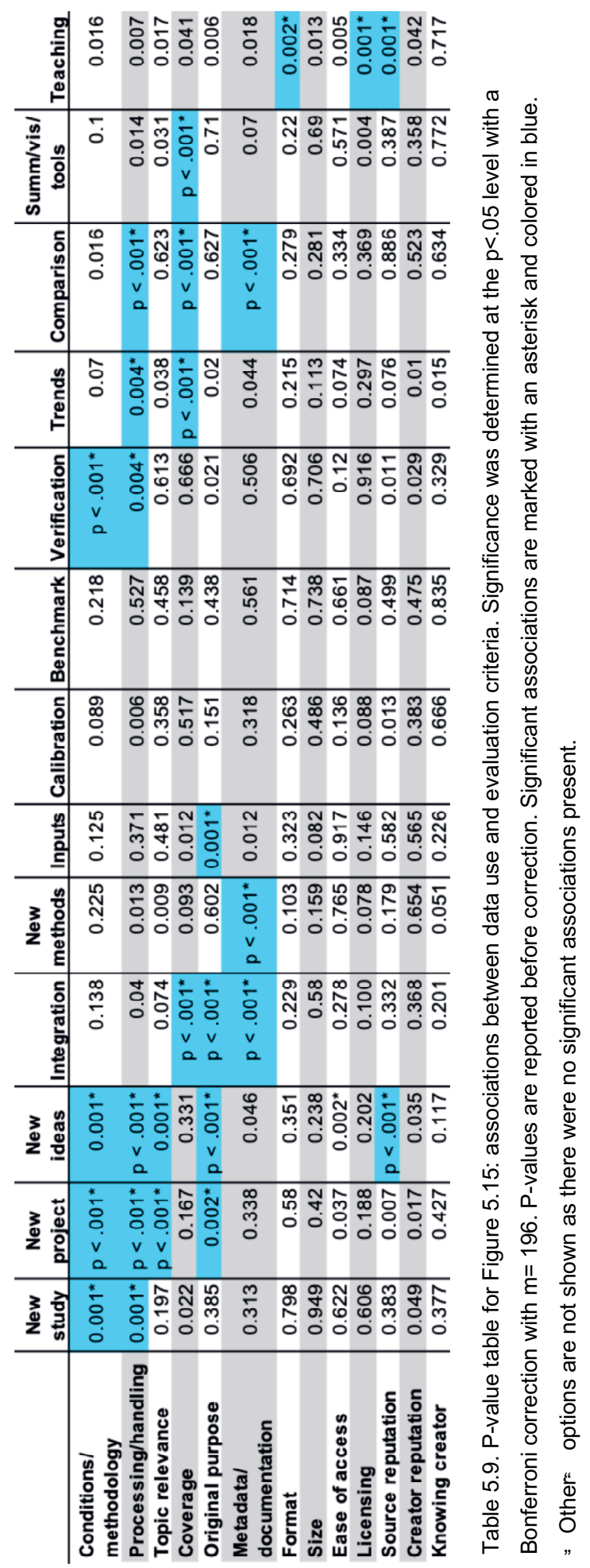


174 | Chapter 5

5.7.3. Appendix C: Sources used in disciplinary subset
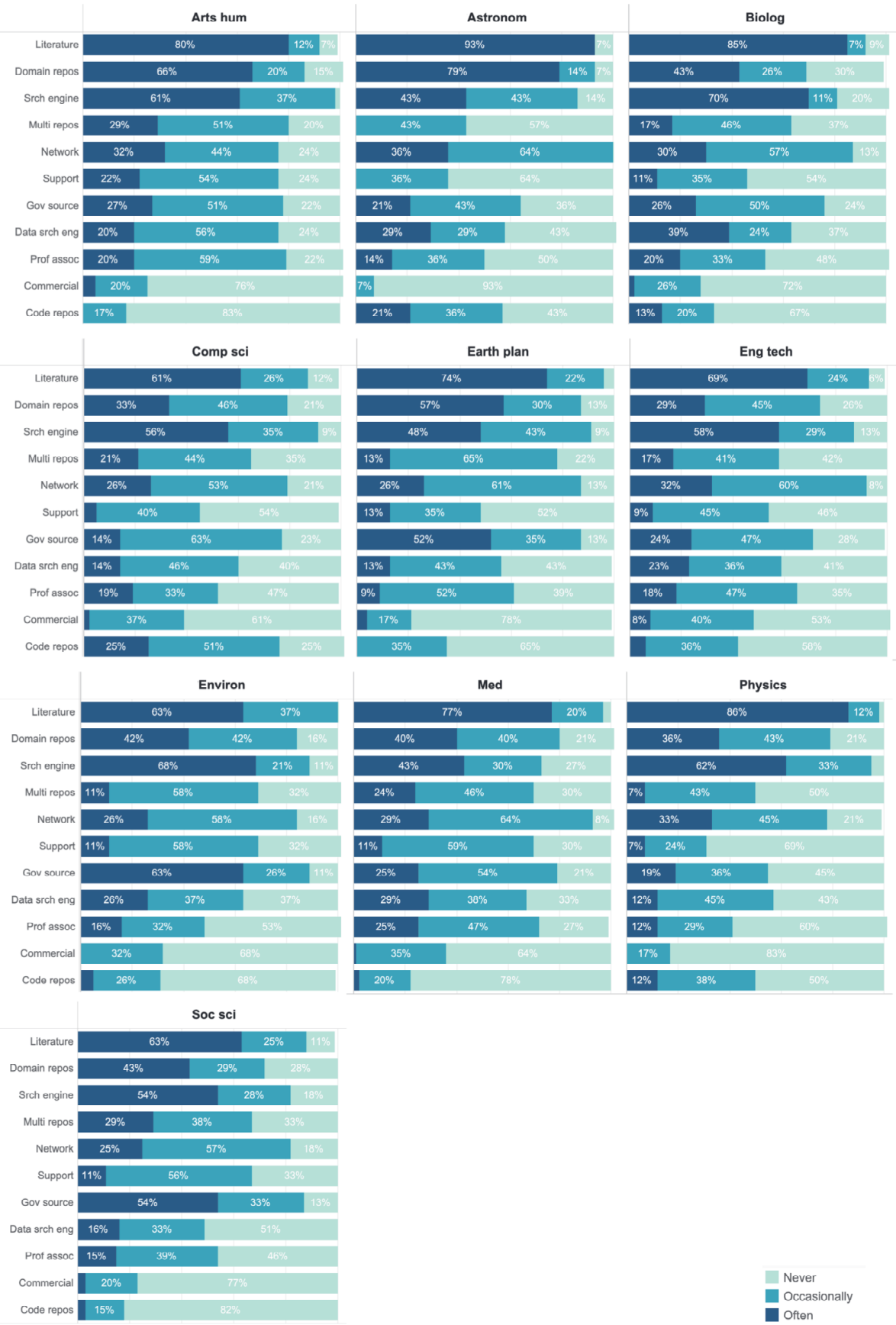

$$
\begin{aligned}
& \text { Never } \\
& \text { Occasionally } \\
& \text { Often }
\end{aligned}
$$

Figure 5.17. Sources used in the disciplinary subset for respondents selecting only one discipline. Percents are percent respondents. Arts \& humanities $(n=43)$; astronomy $(n=14)$; biological science $(n=46)$; computer science $(n=57)$; earth \& planetary science $(n=24)$; engineering \& technology $(n=80)$; environmental science $(n=22)$; medicine $(n=91)$; physics $(n=42)$; social science $(n=81)$. 


\section{CHAPTER 6}




\section{A dataset describing data discovery and reuse practices in research}

Originally published as: Gregory, K. (2020). A dataset describing data discovery and reuse practices in research. Scientific Data, 7(232).

https://doi.org/10.1038/s41597-020-0569-5 


\section{8 | Chapter 6}
Abstract
This paper presents a dataset produced from the largest known survey examining how researchers and support professionals discover, make sense of and reuse secondary research data. 1677 respondents in 105 countries representing a variety of disciplinary domains, professional roles and stages in their academic careers completed the survey. The results represent the data needs, sources and strategies used to locate data, and the criteria employed in data evaluation of these respondents. The data detailed in this paper have the potential to be reused to inform the development of data discovery systems, data repositories, training activities and policies for a variety of general and specific user communities. 


\subsection{Background \& summary}

Reusing data created by others, so-called secondary data (Allen, 2017), holds great promise in research (Wilkinson et al., 2016). This is reflected in the creation of policies (European Commission, 2019); platforms, i.e. the European Open Science Cloud (European Commission, 2017); metadata schemas, i.e. the DataCite schema (DataCite Metadata Working Group, 2019) and search tools, i.e. Google Dataset Search ${ }^{15}$ or DataSearch $^{16}$, to facilitate the discovery and reuse of data. Despite the emergence of these systems and tools, not much is known about how users interact with data in search scenarios (Noy et al., 2019) or the particulars of how such data are used in research (Pasquetto et al., 2017).

This paper describes a dataset first analyzed in the article Lost or Found? Discovering data needed for research (Gregory et al., 2020). The dataset includes quantitative and qualitative responses from a global survey, with 1677 complete responses, designed to learn more about data needs, data discovery behaviors, and criteria and strategies important in evaluating data for reuse. This survey was conducted as part of a project investigating contextual data search undertaken by two universities, a data archive, and an academic publisher, Elsevier. The involvement of Elsevier enabled the recruitment strategy, namely drawing the survey sample from academic authors who have published an article in the past three years that is indexed in the Scopus literature database. ${ }^{17}$ This recruitment strategy helped to ensure that the sample consisted of individuals active in research, across disciplines and geographic locations.

The data themselves are presented in two data files, according to the professional role of respondents. The dataset as a whole consists of these two data files, one for researchers (with 165 variables) and one for research support professionals (with 167 variables), the survey questionnaire and detailed descriptions of the data variables (Gregory, 2020a). The survey questionnaire contains universal questions which could be applicable to similar studies; publishing the questionnaire along with the data files not only facilitates understanding the data, but it also fosters possible harmonization with other survey-based studies.

The dataset has the potential to answer future research questions, some of which are outlined in the usage notes of this paper, and to be applied at a practical level. Designers of

\footnotetext{
${ }^{15} \mathrm{https}: / /$ datasetsearch.research.google.com

16 https://datasearch.elsevier.com

17 https://www.scopus.com
} 
$180,187,217,219,221,228,236,251$ both general and specific data repositories and data discovery systems could use this dataset as a starting point to develop and enhance search and sensemaking interfaces. Data metrics could be informed by information about evaluation criteria and data uses present in the dataset, and educators and research support professionals could build on the dataset to design training activities.

\subsection{Methods}

The description below of the methods used to design the questionnaire and to collect the data, as well as the description of potential biases in the technical validation section, all build on those presented in the author's previous work (Gregory et al., 2020).

\subsubsection{Questionnaire design}

The author's past empirical work investigating data search practices (Gregory, Groth, et al., 2019; Gregory, Cousijn, et al., 2019; see also Figure 6.1), combined with established models of interactive information retrieval (i.e. Ingwersen, 1992; 1996; Belkin, 1993; 1996) and information seeking (Blandford \& Attfield, 2010; Adams \& Blandford, 2005) and other studies of data practices (i.e. Borgman, 2015a; Faniel \& Yakel, 2017), were used to design questions examining the categories identified in Table 6.1. Specifically, questions explored respondents' data needs, their data discovery practices, and their methods for evaluating and making sense of secondary data.

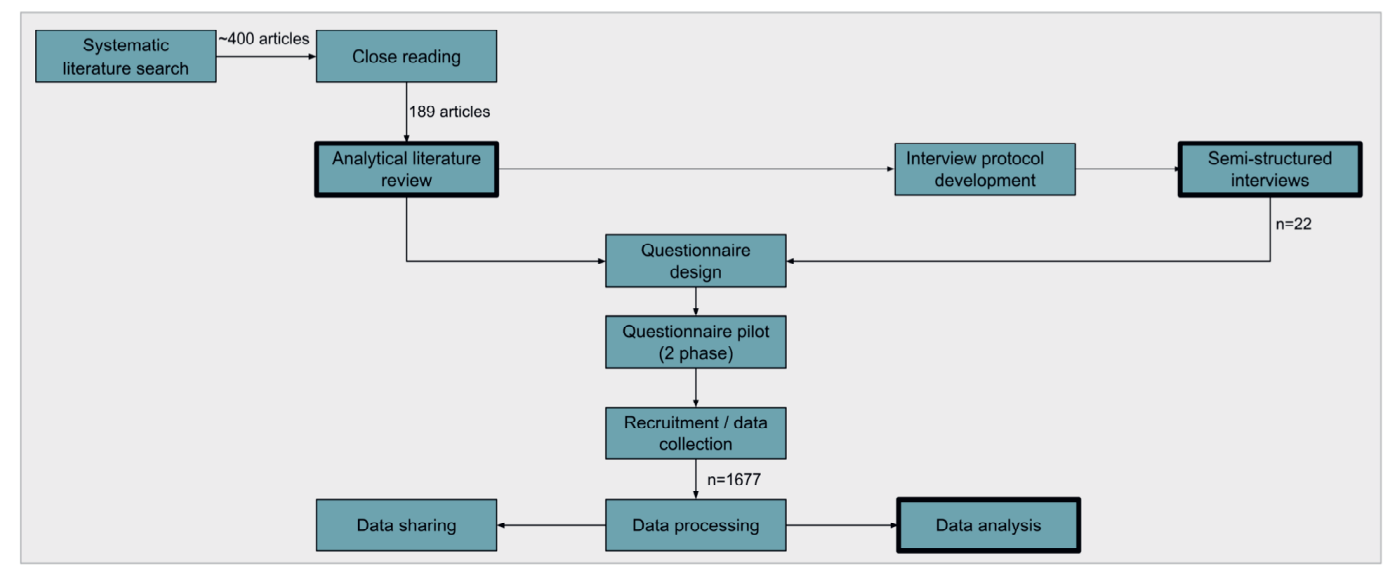

Figure 6.1. Creation of dataset in relation to prior empirical work by the author. Bolded rectangles indicate steps with associated publications, resulting from an analytical literature review (Gregory, Groth, et al., 2019), semi-structured interviews (Gregory, Cousijn, et al., 2019) and an analysis of the survey data (Gregory et al., 2020). 
The questionnaire used a branching design, consisting of a maximum of 28 primarily multiple choice items (Table 6.1). The final question of the survey, which provided space for respondents to provide additional comments in an open text field is not represented in Table 6.1. The individual items were constructed in accordance with best practices in questionnaire design, with special attention given to conventions for wording questions and the construction of Likert scale questions (de Vaus, 2013; Robson \& McCartan, 2016). Nine of the multiple choice questions were constructed to allow multiple responses. There were a maximum of three optional open response questions. The majority of multiple choice questions also included the possibility for participants to write-in an "other" response.

The first branch in the questionnaire design was based on respondents' professional role. Respondents selecting "librarians, archivists or research/data support providers," a group referred to here as research support professionals, answered a slightly different version of the questionnaire. The items in this version of the questionnaire were worded to reflect possible differences in roles, i.e. whether respondents seek data for their own use or to support other individuals. Four additional questions were asked to research support professionals in order to further probe their professional responsibilities; four questions were also removed from this version of the questionnaire. This was done in order to maintain a reasonable completion time for the survey and because the removed questions were deemed to be more pertinent to respondents with other professional roles, i.e. researchers.

The questionnaire is available in its entirety with the rest of the dataset (Gregory, 2020a).

\begin{tabular}{|c|c|c|}
\hline Category & Content & Branches \\
\hline Demographics & $\begin{array}{l}\text { Role } \\
\text { Discipline* } \\
\text { Years professional experience } \\
\text { Country of employment } \\
\text { Organization type } \\
\text { Perceptions (own, others) about data sharing } \\
\text { Perceptions (own, others) about data reuse } \\
\text { Ever shared data (R only) }\end{array}$ & $\begin{array}{l}\text { If research support professional (RSP) - asked all questions } \\
\text { plus those marked } R S P \text { only; not asked questions marked } R \\
\text { only } \\
\text { If not RSP - asked all questions plus those marked } R \text { only; } \\
\text { not asked questioned marked } R S P \text { only }\end{array}$ \\
\hline Data needs & $\begin{array}{l}\text { Describe neөded data } \\
\text { Select data type needed* } \\
\text { Purpose for using secondary data* } \\
\text { Need data outside of discipline ( } R \text { only) } \\
\text { Need data to support others/own research (RSP only) }\end{array}$ & $\begin{array}{l}\text { If yes - how find these data (R only) } \\
\text { If support others - Who do you support* (RSP only) } \\
\qquad \begin{array}{l}\text { - How do you provide support* (RSP only) }\end{array}\end{array}$ \\
\hline & $\begin{array}{l}\text { Who finds data for you* (R only) } \\
\text { Sources used to find data - frequency of use }\end{array}$ & $\begin{array}{l}\text { If academic literature - how use literature?* } \\
\text { If search engine - how successful? }\end{array}$ \\
\hline Discovering & $\begin{array}{l}\text { Active vs serendipitous data discovery } \\
\text { Social interactions in discovery and access }{ }^{\star \star} \\
\text { Discover literature differently than data } \\
\text { Ease of finding data ( } R \text { only) }\end{array}$ & $\begin{array}{l}\text { If not no - describe differences } \\
\text { If not easy - what are the challenges?* }\end{array}$ \\
\hline $\begin{array}{l}\text { Evaluating } / \\
\text { sensemaking }\end{array}$ & $\begin{array}{l}\text { Social interactions in evaluating/sensemaking }{ }^{* *} \\
\text { Important information about data when evaluating } \\
\text { Differences between self and those support (RSP only) } \\
\text { Evaluation/sensemaking strategies } \\
\text { Important aspects in establishing trust } \\
\text { Important aspects in establishing quality }\end{array}$ & \\
\hline
\end{tabular}


Table 6.1. Content and branches of questionnaire items listed according to questionnaire section. Questions only asked to research support professionals are marked with RSP; those only asked to other respondents are marked with $\mathrm{R}$. Items that allowed multiple responses are marked with an asterisk. Items marked with a double asterisk correspond to the same multiple response question.

\subsubsection{Sampling, recruitment and administration}

Individuals involved in research, across disciplines, who seek and reuse secondary data comprised the population of interest. This is a challenging population to target, as it is difficult to trace instances of data reuse, particularly given the fact that data citation, and other forms of indexing, are still in their infancy (Park et al., 2018). The data reuse practices of individuals in certain disciplines have been better studied than others (Borgman et al., 2020), in part because of the existence of established data repositories within these disciplines (Leonelli, 2013). In order to recruit individuals active in research across many disciplinary domains, a broad recruitment strategy was adopted.

Recruitment emails were sent to a random sample of 150,000 authors who are indexed in Elsevier's Scopus database and who have published in the past three years. The recruitment sample was created to reflect the distribution of published authors by country within Scopus. Two batches of recruitment emails were sent: one of 100,000 and the other of 50,000. One reminder email was sent two weeks after the initial email. A member of the Elsevier Research and Academic Relations team created the sample and sent the recruitment letter, as access to the email addresses was not available to the investigator due to privacy regulations. The questionnaire was scripted and administered using the Confirmit software. ${ }^{18}$

1637 complete responses were received during a four-week survey period between September and October 2018 using this methodology. Only seven of the 1637 responses came from research support professionals. In a second round of recruitment in October 2018, messages were posted to discussion lists in research data management and library science to further recruit support professionals. Individuals active in these lists spontaneously posted notices about the survey on their own Twitter feeds. These methods resulted in an additional 40 responses, yielding a total of 1677 complete responses.

\footnotetext{
${ }^{18}$ https://www.confirmit.com
} 


\subsubsection{Ethical review and informed consent}

This study was approved by the Ethical Review Committee Inner City faculties (ERCIC) at Maastricht University, Netherlands, on 17 May 2018 under the protocol number ERCIC_078_01_05_2018.

Prior to beginning the study, participants had the opportunity to review the informed consent form. They indicated their consent by clicking on the button to proceed to the first page of survey questions. Respondents were informed about the purpose of the study, its funding sources, the types of questions which would be asked, how the survey data would be managed and any foreseen risks of participation.

Specifically, respondents were shown the text below, which also states that the data would be made available in the DANS-EASY data repository, ${ }^{19}$ which is further described in the Data Records section of this paper.

Your responses will be recorded anonymously, although the survey asks optional questions about demographic data which could potentially be used to identify respondents. The data will be pseudonymized (e.g. grouping participants within broad age groups rather than giving specific ages) in order to prevent identification of participants. The results from the survey may be compiled into presentations, reports and publications. The anonymized data will be made publicly available in the DANSEASY data repository.

Respondents were also notified that participation was voluntary, and that withdrawal from the survey was possible at any time. They were further provided with the name and contact information of the primary investigator.

\subsection{Data records}

\subsubsection{Preparation of data files}

The data were downloaded from the survey administration system as csv files by the employee from Elsevier and were sent to the author. The downloads were performed in two batches: the 1637 responses received before the additional recruiting of research support professionals, and the 40 responses received after this second stage of recruitment. The

\footnotetext{
${ }^{19}$ https://easy.dans.knaw.nl
} 
seven responses from research support professionals from the first round of recruitment were extracted and added to the csv file from the second batch. This produced separate files for research support professionals and the remainder of respondents, who are referred to as researchers in this description. This terminology is appropriate as the first recruitment strategy ensured that respondents were published academic authors, making it likely that they had been involved in conducting research at some point in the past three years.

The following formatting changes were made to the data files in order to enhance understandability for future data reusers. All changes were made using the analysis program R (R Core Team, 2017).

- Open responses were checked for any personally identifiable information, particularly email addresses. This was done by searching for symbols and domains commonly used in email addresses (i.e. “@”; “.com,” and “.edu”). Two email addresses were identified in the final question recording additional comments about the survey. In consultation with an expert at the DANS-EASY data repository, all responses from this final question were removed from both data files as a precautionary measure.

- Variables representing questions asked only to research support professionals were removed from datadiscovery_researchers.csv. Variables representing questions asked only to researchers were removed from datadiscovery_supportprof.csv.

- Variables were renamed using mnemonic names to facilitate understanding and analysis. Variable names for questions asked to both research support professionals and researchers have the same name in both data files.

- Variables were re-ordered to match the order of the questions presented in the questionnaire. Demographic variables, including role, were grouped together at the end of the data files.

- Multiple choice options which were not chosen by respondents were recorded by the survey system as zeros. If a respondent was not asked a question, this is coded as "Not asked." If a respondent wrote "NA" or a similar phrase in the open response questions, this was left unchanged to reflect the respondent's engagement with the survey. If a respondent did not complete an optional open response question, this was recorded as a space, which appears as an empty cell. In the analysis program R, e.g., this empty space is represented as " ". 


\subsubsection{Description of data and documentation files}

The dataset described here consists of one text readme file, four csv files, and one pdf file with the survey questionnaire. These files should be used in conjunction with each other in order to appropriately use the data. Table 6.2 provides a summary and description of the files included in the dataset.

\begin{tabular}{|c|c|c|c|}
\hline Data file name & Description & No. cases & No. variables \\
\hline GREGORY_DATA_DISCOVERY_Readme.txt & Provides guidance to the dataset & $N A$ & $N A$ \\
\hline datadiscovery_researchers.csv & $\begin{array}{l}\text { Contains data for respondents identifying } \\
\text { themselves as researchers, students, } \\
\text { managers or other types of professionals }\end{array}$ & 1630 & 165 \\
\hline datadiscovery_supportprof.csv & $\begin{array}{l}\text { Contains data for respondents identifying } \\
\text { themselves as librarians, archivists or } \\
\text { research/data support providers }\end{array}$ & 47 & 167 \\
\hline variable_labels_researchers.csv & $\begin{array}{l}\text { Contains a description of the variable } \\
\text { names in the } \\
\text { datadiscovery_researchers.csv file }\end{array}$ & $N A$ & $N A$ \\
\hline variable_labels_supportprof.csv & $\begin{array}{l}\text { Contains a description of the variable } \\
\text { names in the } \\
\text { datadiscovery supportprof.csv }\end{array}$ & NA & $N A$ \\
\hline datadiscovery_questionnaire.pdf & $\begin{array}{l}\text { Contains the questionnaire for both } \\
\text { researchers and research support } \\
\text { professionals }\end{array}$ & NA & NA \\
\hline
\end{tabular}

Table 6.2. Description of files composing the dataset.

Descriptions of the variable names are provided in two files (Table 6.2). Variables were named following a scheme that matches the structure of the questionnaire; each variable name begins with a mnemonic code representing the related research aim. The primary codes are summarized in Table 6.3. The values of the variables for multiple choice items are represented as either a "0" for non-selected options, as described above, or with a textual string representing the selected option.

\begin{tabular}{|c|c|}
\hline Mnemonic code & Associated research aim \\
\hline need_ & Data needs \\
\hline use_ & Purposes for which data are used \\
\hline find_ & Data search and discovery practices \\
\hline strategy_ & $\begin{array}{l}\text { Data discovery strategies } \\
\text { Related to find }\end{array}$ \\
\hline source_ & $\begin{array}{l}\text { Sources used to discover data } \\
\text { Related to find_ }\end{array}$ \\
\hline eval__ & Data evaluation and sense-making \\
\hline accss_ & Data access \\
\hline disc_ & Discipline chosen \\
\hline dem_ & Demographic information \\
\hline
\end{tabular}


Table 6.3. Description of primary mnemonic codes used to preface variable names

The dataset is available at the DANS-EASY data repository. DANS-EASY is a principal component of the federated national data infrastructure of the Netherlands (Dillo \& Doorn, 2014) and is operated by the Data Archive and Networked Services (DANS), an institution of the Royal Netherlands Academy for Arts and Sciences and the Dutch Research Council. DANS-EASY has a strong history of providing secure long-term storage and access to data in the social sciences Doorn, 2020). The repository has been awarded a CoreTrustSeal certification for data repositories, ${ }^{20}$ which assesses the trustworthiness of repositories according to sixteen requirements. These requirements focus on organizational infrastructure (e.g. licences, continuity of access and sustainability), digital object management (e.g. integrity, authenticity, preservation, and re-use) and technology (e.g. technical infrastructure and security).

\subsubsection{Sample characteristics}

Respondents identified their disciplinary domains of specialization from a list of 31 possible domains developed after the list used by Berghmans, et al (2017). Participants could select multiple responses for this question. The domain selected most often was engineering and technology, followed by the biological, environmental and social sciences (Figure 6.2a) Approximately half of the respondents selected two or more domains, with one quarter selecting more than three.

Forty percent of respondents have been professionally active for 6-15 years (Figure 6.2b). The majority identified as being researchers $(82 \%)$ and are employed at universities $(69 \%)$ or research institutions (17\%). Respondents work in 105 countries; the most represented countries include the United States, Italy, Brazil and the United Kingdom (Figure 6.2c).

${ }^{20} \mathrm{https}: / / \mathrm{www}$. coretrustseal.org 

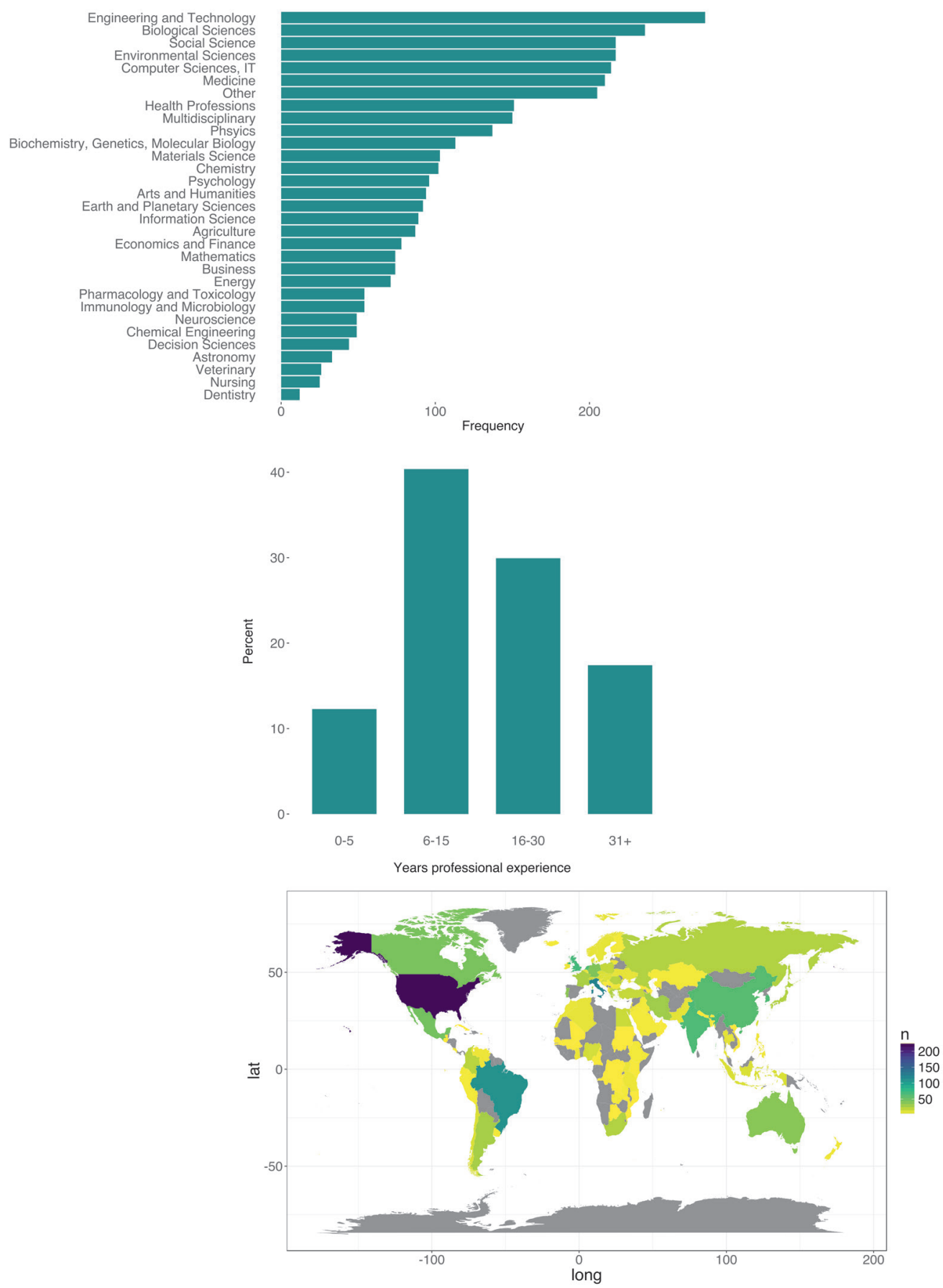

Figure 6.2. a) Disciplinary domains selected by respondents; multiple responses possible ( $n=3431)$. b) Respondents' years of professional experience; percentages denote percent of respondents $(n=1677)$. c) Number of respondents by country of employment $(n=1677)$. 


\subsection{Technical validation}

Several measures were performed to ensure the validity of the data, both before and after data collection. Sources of uncertainty and potential bias in the data are also outlined below in order to facilitate understanding and data reuse.

\subsubsection{Questionnaire development}

The questionnaire items were developed after extensively reviewing relevant literature (Gregory, Groth, et al., 2019; Kim \& Yoon, 2017; Kratz \& Strasser, 2015; Schmidt et al., 2016; Tenopir et al., 2015) and conducting semi-structured interviews to test the validity of our guiding constructs. To test the validity and usability of the questionnaire itself, a twophase pilot study was conducted. In the first phase, four researchers, recruited using convenience sampling, were observed as they completed the online survey. During these observations, the researchers "thought out loud" as they completed the survey; they were encouraged to ask questions and to make remarks about the clarity of wording and the structure of the survey. Based on these comments, the wording of questions was finetuned and additional options were added to two multiple choice items.

In the second pilot phase, an initial sample of 10,000 participants was recruited, using the primary recruitment methodology detailed in the methods section of this paper. After 102 participants interacted with the survey, the overall completion rate $(41 \%)$ was measured and points where individuals stopped completing the survey were noted. Based on this information, option-intensive demographic questions (i.e. country of employment, discipline of specialization) were moved to the final section of the survey in order to minimize survey fatigue. The number of open-ended questions were also reduced and open-response questions were made optional.

The online presentation of the survey questions also helped to counter survey fatigue. Only one question was displayed at a time; the branching logic of the survey ensured that respondents were only shown the questions which were relevant to them, based on their previous answers.

\subsubsection{Questionnaire completion}

1677 complete responses to the survey questionnaire were received. Using the total number of recruitment emails in the denominator, this yields a response rate of $1.1 \%$. Taking into account the number of non-delivery reports which were received $(29,913)$, the number of 
invalid emails which were reported (81) and the number of recruited participants who elected to opt-out of the survey (448) yields a slightly higher response rate of $1.4 \%$. It is likely that not all of the 150,000 individuals who received recruitment emails match our targeted population of data seekers and reusers. Knowledge about the individuals who did not respond to the survey and about the frequency of data discovery and reuse within research as a whole, is limited; this complicates the calculation of a more accurate response rate, such as the methodology described in (American Association for Public Opinion Research, 2016).

A total of 2,306 individuals clicked on the survey link, but did not complete it, yielding a completion rate of $42 \%$. Of the non-complete responses, fifty percent stopped responding after viewing the introduction page with the informed consent statement. This point of disengagement could be due to a variety of reasons, including a lack of interest in the content of the survey or a disagreement with the information in the consent form. The majority of individuals who did not complete the survey stopped responding within the first section of the survey ( $75 \%$ of non-complete responses). Only data from complete responses are included in this dataset.

Of the 1677 complete responses, there was a high level of engagement with the optional open response questions. Seventy-eight percent of all respondents answered Q2 regarding their data needs; $92 \%$ of respondents who were asked Q5a provided an answer; and $69 \%$ of respondents shown Q10a described how their processes for finding academic literature and data differ.

\subsubsection{Data quality and completeness}

Checks for missing values and NAs were performed using standard checks in R. As detailed in the section on data preparation, multiple choice responses not selected by respondents were recorded as a zero. If a respondent was not asked a question, this was coded as "Not asked." If a respondent wrote "NA" or a similar phrase in the open response questions, this was left unchanged to reflect the respondent's engagement with the survey. If a respondent did not complete an optional open response question, this was recorded as a space, which appears as an empty cell. In the analysis program R, e.g., this empty space is represented as " ".

Due to the limited available information about non-responders to the survey and about the frequency of data seeking and discovery behaviors across domains in general, the data as 
they stand are representative only of the behaviors of our nearly 1700 respondents - a group of data-aware people already active in data sharing and reuse and confident in their ability to respond to an English-language survey. Surveys in general tend to attract a more active, communicative part of the targeted population and do not cover non-users at all (Wyatt, 2003). While not generalizable to broader populations, the data could be transferable (Lincoln \& Guba, 1985; Firestone, 1993) to similar situations or communities. Creating subsets of the data, i.e. by discipline, may provide insights that can be applied to particular disciplinary communities.

There are potential sources of bias in the data. The recruited sample was drawn to mirror the distribution of published authors by country in Scopus; the geographic distribution of respondents does not match that of the recruited sample (Table 6.4). This is especially noticeable for Chinese participants, who comprised $15 \%$ of the recruited sample, but only $4 \%$ of respondents. This difference could be due to a number of factors, including language differences, perceived power differences (Harzing, 2006), or the possibility that data seeking is not a common practice.

\begin{tabular}{lc|lc}
\hline \multicolumn{2}{c|}{ Percent of recruited sample } & \multicolumn{2}{c}{ Percent of respondents } \\
\hline United States & $19 \%$ & United States & $13 \%$ \\
China & $15 \%$ & Italy & $7 \%$ \\
United Kingdom & $5 \%$ & Brazil & $7 \%$ \\
Germany & $5 \%$ & United Kingdom & $4 \%$ \\
Japan & $4 \%$ & India & $4 \%$ \\
France & $4 \%$ & South Korea & $4 \%$ \\
India & $4 \%$ & Netherlands & $4 \%$ \\
Italy & $3 \%$ & China & $4 \%$ \\
Canada & $3 \%$ & Mexico & $3 \%$ \\
Spain & $3 \%$ & Canada & $3 \%$ \\
Australia & $3 \%$ & Germany & $3 \%$ \\
South Korea & $3 \%$ & Australia & $2 \%$ \\
Brazil & $2 \%$ & Portugal & $2 \%$ \\
Netherlands & $2 \%$ & Iran & $2 \%$ \\
Russian Federation & $1 \%$ & Argentina & $2 \%$ \\
Taiwan & $1 \%$ & France & $2 \%$ \\
Iran & $1 \%$ & Greece & $1 \%$ \\
Swizerland & $1 \%$ & Japan & $1 \%$ \\
Turkey & $1 \%$ & Russian Federation & $1 \%$ \\
Poland & $1 \%$ & South Africa & $1 \%$ \\
Other & $19 \%$ & Romania & $1 \%$ \\
\hline
\end{tabular}

Table 6.4. Percentage of recruited participants by geographic location compared to percentage of respondents providing complete responses.

Our respondents were primarily drawn from the pool of published authors in the Scopus database. Some disciplinary domains are under-represented within Scopus, most notably 
the arts and humanities (Mongeon \& Paul-Hus, 2016; Vera-Baceta et al., 2019). Subject indexing within Scopus occurs at the journal or source level. As of January $2020,30.4 \%$ of titles in Scopus are from the health sciences; $15.4 \%$ from the life sciences; $28 \%$ from the physical sciences and $26.2 \%$ from the social sciences ${ }^{45}$. Scopus has an extensive and welldefined review process for journal inclusion; $10 \%$ of the approximately 25,000 sources indexed in Scopus are published by Elsevier (Elsevier, 2020).

Self-reported responses also tend to be pro-attitudinal, influenced by a respondent's desire to provide a socially acceptable answer. Survey responses can also be influenced by the question presentation, wording and multiple choice options provided. The pilot studies and the provision of write-in options for individual items helped to mitigate this source of error.

\subsection{Usage notes}

\subsubsection{Notes for data analysis}

- It is key to note which questions were designed to allow for multiple responses. This will impact the type of analysis which can be performed and the interpretation of the data. These nine questions are marked with an asterisk in Table 6.1; the names of the variables related to these questions are summarized in Table 6.5.

\begin{tabular}{|c|c|}
\hline Question content & Variable names \\
\hline Discipline & all variables beginning with code disc \\
\hline Data type needed & need_obs, need_exp, need_sim, neèd_deriv, \\
\hline Purpose for using secondary data & $\begin{array}{l}\text { use_calb, use_bmk, use_vrf, use_inpt, use_idea, } \\
\text { use_tch, use_nwprj, use_nwmth, use_tnd, } \\
\text { use_cmp, use_smvs, use_intg, use_oth }\end{array}$ \\
\hline Who do you support? (RSP) & $\begin{array}{l}\text { whosupprt_stud, whosupprt_res, whosupprt_indus, } \\
\text { whosupprt_oth }\end{array}$ \\
\hline How do you support? (RSP) & $\begin{array}{l}\text { supprt_teachdmp, supprt_teachsklls, } \\
\text { supprt_finddta, supprt_curate, supprt_findlit, }\end{array}$ \\
\hline Who finds data for you? $(R)$ & $\begin{array}{l}\text { find_whoself, find_whograd, find_whosuppt, } \\
\text { find_whonetwk, find_whooth }\end{array}$ \\
\hline $\begin{array}{l}\text { How do you use academic } \\
\text { literature? }\end{array}$ & $\begin{array}{l}\text { strategy_goal, strategy_serend, strategy_cit, } \\
\text { strategy_extrct, strategy_oth }\end{array}$ \\
\hline What are challenges? & $\begin{array}{l}\text { find_chalaccs, find_chalskill, find_chaldistrb, } \\
\text { find_chaldigtl, find_chaltools, find_chalnetwk, } \\
\text { find_chaloth }\end{array}$ \\
\hline $\begin{array}{l}\text { Social interactions in finding, } \\
\text { accessing, evaluation/sensemaking }\end{array}$ & $\begin{array}{l}\text { find_netwk, find_creatr, find_collab, find_conf, } \\
\text { find_list, accss_netwk, accss_creatr, accss_collab, } \\
\text { accss_conf, accss_list, eval_netwk, eval_creatr, } \\
\text { eval_collab, eval conf, eval list }\end{array}$ \\
\hline
\end{tabular}


Table 6.5. Content of questions which were designed to allow multiple responses and their associated variables. RSP indicates questions only asked to research support professionals. R indicates questions only present in the researcher data file.

- The data are available in standard csv formats and may be imported into a variety of analysis programs, including $\mathrm{R}$ and Python. The data are well-suited in their current form to be treated as factors or categories in these programs, with the exception of open response questions and the write-in responses to the "other" selection options, which should be treated as character strings. An example of the code needed to load the data into $\mathrm{R}$ and Python, as well as how to change the open and other response variables to character strings, is provided in the section on code availability. To further demonstrate potential analyses approaches, the code used to create Figure $6.2 \mathrm{a}$ in $\mathrm{R}$ is also provided.

- Certain analysis programs, i.e. SPSS, may require that the data be represented numerically; responses in the data files are currently represented in textual strings. The survey questionnaire, which is available with the data files, contains numerical codes for each response which may be useful in assigning codes for these variables.

- Future users may wish to integrate the two data files to examine the data from all survey respondents together. This can easily be done by creating subsets of the variables of interest from each data file (i.e. by using the subset and select commands in R) and combining the data into a single data frame (i.e. using the rbind command in R). Variables that are common between both of the data files have the same name, facilitating this type of integration. An example of the code needed to do this is provided in the code for creating Figure 6.2a.

- Open and write-in responses are included in the same data file with the quantitative data. These variables can be removed and analyzed separately, if desired.

- To ease computational processing, the data do not include embedded information about the question number or the detailed meaning of each variable name. This information is found in the separate variable_labels csv file associated with each data file. 


\subsubsection{Potential questions and applications}

The data have the potential to answer many interesting questions, including those identified below.

1) How do the identified practices vary by demographic variables? The data could be sub-setted to examine practices along the lines of:

- Country of employment

- Career stage, e.g. early career researchers

- Disciplinary domain

2.) What correlations exist among the different variables, particularly the variables allowing for multiple responses? Such questions could examine:

- Possible correlations between the frequency of use of particular sources and the type of data needed or uses of data

- Possible correlations between particular challenges for data discovery and needed data or data use

3.) How representative are these data of the behaviors of broader populations?

4.) How will these behaviors change as new technologies are developed? The data could serve as a baseline for comparison for future studies.

5.) How do practices within a particular domain relate to the existence of data repositories and infrastructures within a domain? Given the practices identified in this survey, how can repositories and infrastructures better support data seekers and reusers?

\subsection{Code Availability}

All R scripts used in data preparation and technical validation, along with the un-prepared data, are available upon request from the corresponding author. Examples of how to load the data and how to change factor/category columns to character columns in R (Box 6.1) and Python (Box 6.2) are provided below. Additionally, the code used to create Figure 6.2a 
in $\mathrm{R}$ (Box 6.3) is listed as an example of how to combine data from both data files into a single plot.

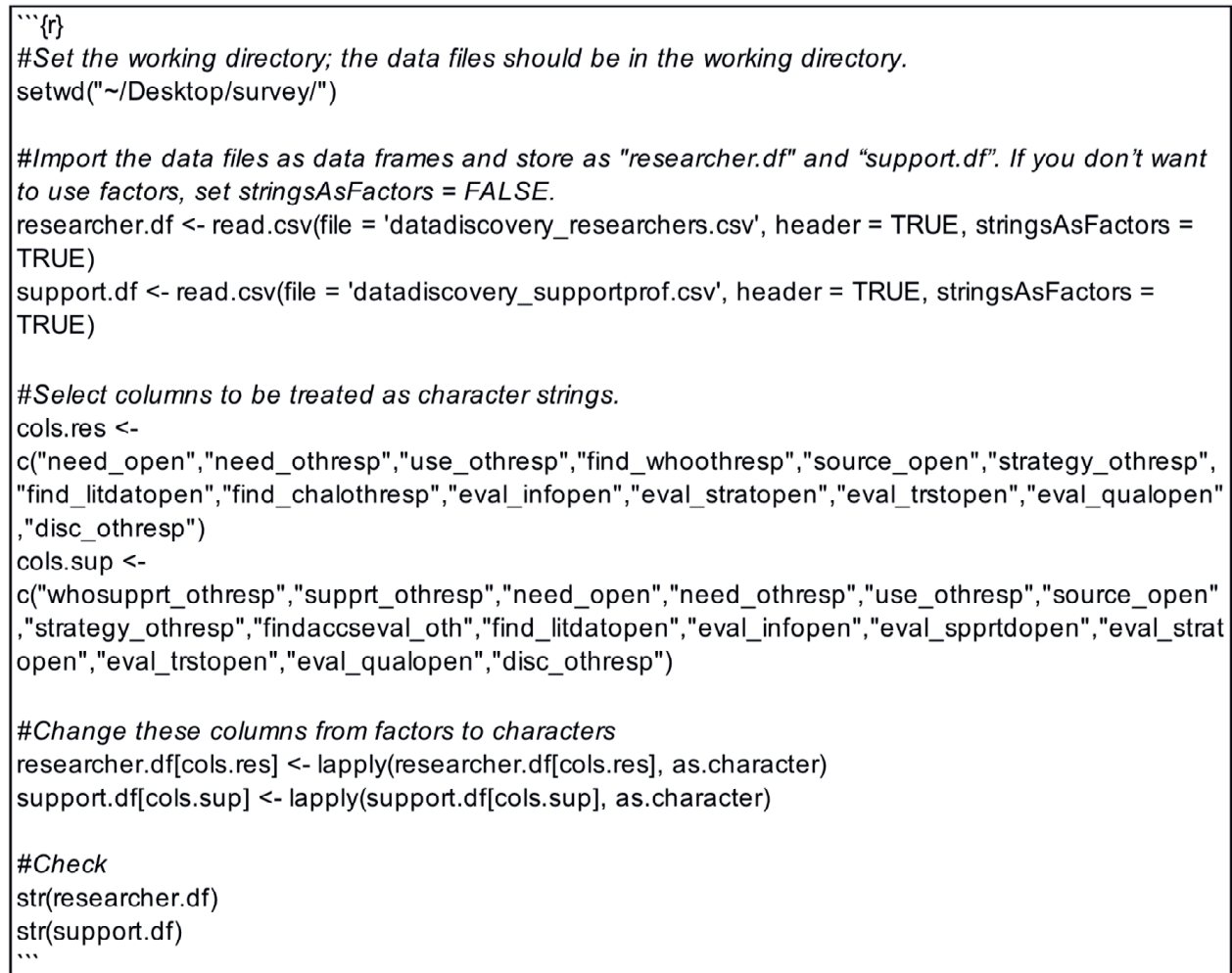

Box 6.1. $\mathrm{R}$ code for loading data and changing selected columns to character strings. 


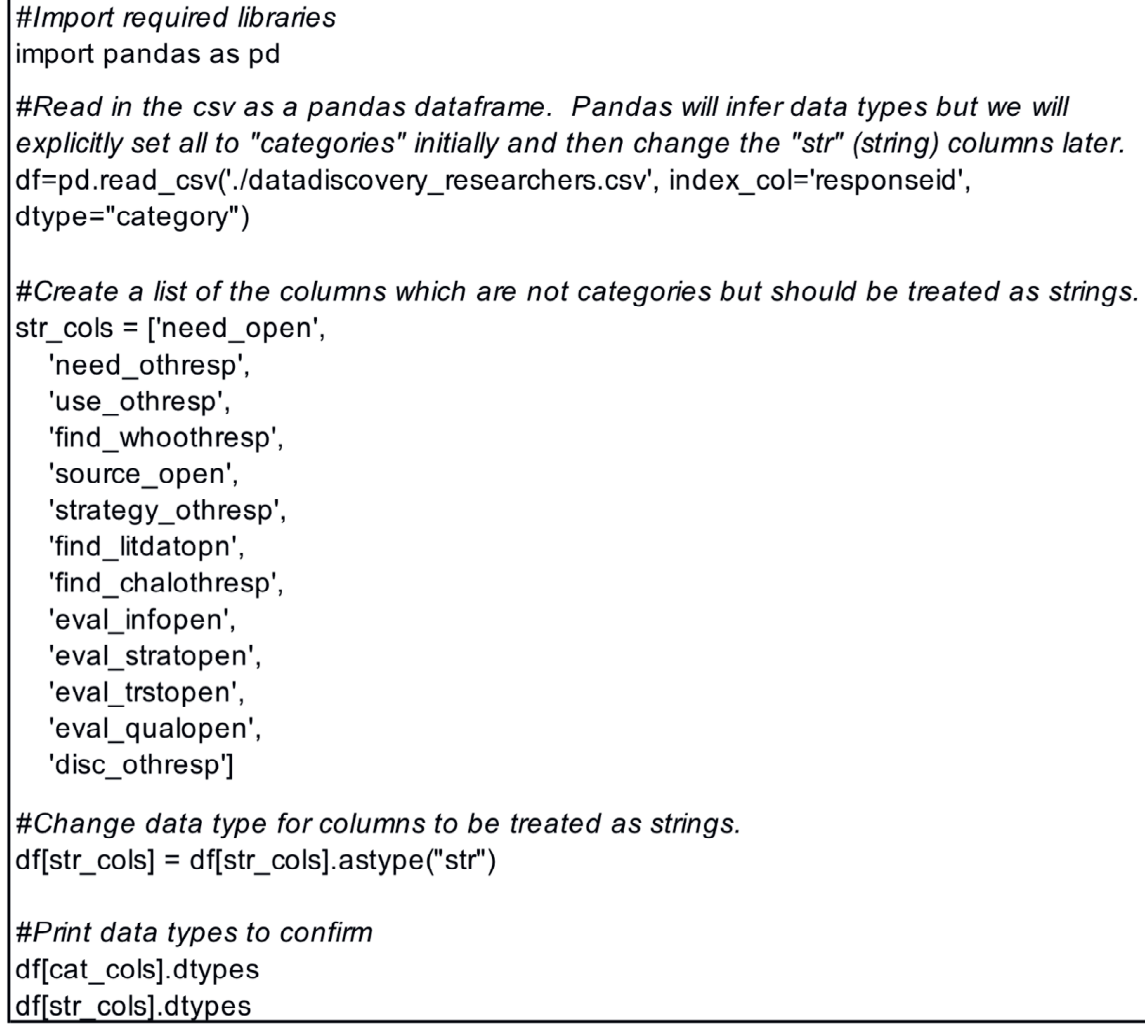

Box 6.2. Python code for loading data and changing selected columns to character strings. 


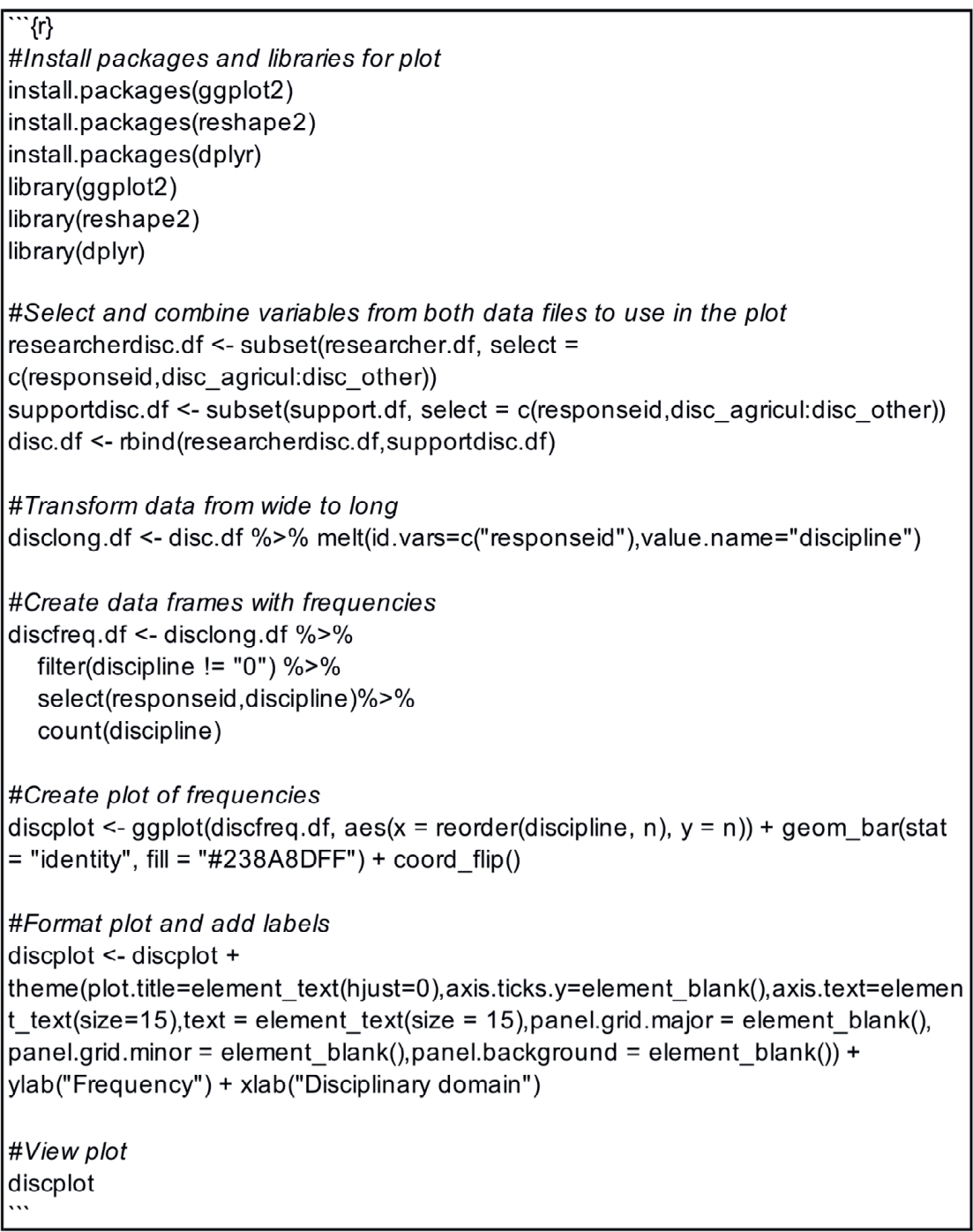

Box 6.3. $R$ code for creating Figure 6.2a.

\section{Acknowledgements}

Paul Groth, Andrea Scharnhorst and Sally Wyatt provided valuable feedback and comments on this paper. I also wish to acknowledge Ricardo Moreira for his assistance in creating the sample and distributing the survey, Wouter Haak for his organizational support, Helena Cousijn for her advice in designing the survey, and Emilie Kraaikamp for her advice regarding personally identifiable information.

This work is part of the project Re-SEARCH: Contextual Search for Research Data and was funded by the NWO Grant 652.001.002. 


\subsection{Reflection: On data papers: Toil, duplication and}

\section{standardization}

Data publishing is not a new phenomenon. Data have been published within handbooks in the chemical and material sciences for over a hundred years, and more recently, as supplements to journal articles. Amidst the development of new technologies, policies, and ways of doing science, new possibilities for making data public are emerging, including the creation of so-called data papers. In this section I briefly explain the structure of data papers and their proposed benefits, before reflecting on my experience of writing the data paper reprinted in this chapter.

\subsubsection{The what and why of data papers}

A data paper is, first and foremost, a paper. It is published in a journal, and aims to document the "what, where, why, how and who" of a particular dataset (Callaghan et al., 2012, p.112). Although data papers are published in journals, the data which they describe are hosted in a repository where they are (hopefully) securely archived for the long term (Figure 6.3).

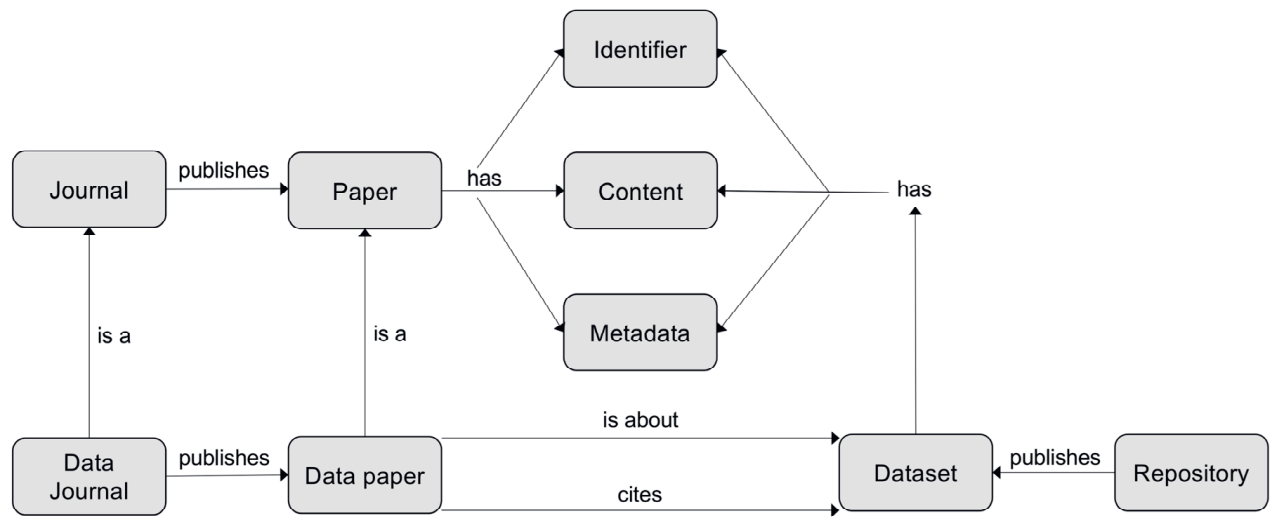

Figure 6.3. Data papers concept map, adapted from Candela et al., 2015.

Both the data and the data paper have associated metadata and persistent identifiers, providing the underlying structure for some of the proposed benefits of this format (see Kratz \& Strasser, 2014 for an overview). The metadata and identifiers of the data paper should facilitate search and discovery within common literature databases and resources; they should also enable standardized, stable data citation. Data papers have the potential to facilitate reuse, not only by making data more findable, but also by providing rich 
descriptions, beyond those in associated articles or metadata. Data papers are peerreviewed, providing another potential benefit, as a positive decision in the peer review process could be taken as an indication of the quality of the data themselves.

Both the number of data papers and data journals are increasing, as is the variety of ways in which they are implemented (Candela et al., 2015; Schöpfel et al., 2019). Papers are formatted according to different templates, requiring different contextual information about the described data (Kim, 2020). Data journals are organized and hosted not only by traditional publishers but also in collaboration with data repositories, i.e. the Research Data Journal for the Humanities and Social Sciences, ${ }^{21}$ and umbrella organizations, i.e. CODATA's Data Science Journal22. Some data journals publish only data papers, others offer a mix of traditional articles alongside data papers.

The data paper reprinted in this chapter was published in a mixed data journal, which publishes both data descriptors as well as articles addressing topics relevant to research and scientific data. My reflections about the process of writing this paper fall along three themes: toil, where I discuss the work required to prepare and share data in this format; the affordances and duplication problems data papers present; and the drawbacks and benefits of implementing standard formats.

\subsubsection{Toil}

This chapter represents the end of a much longer process, which involved preparing, documenting and depositing data. The work required to go from data preparation to data paper was often tedious and highly detailed, although not highly interesting. It was a messy process, involving difficult questions which did not have clear-cut answers.

Which data should I share? Should I share the data as they were recorded by the survey system? Or should I share the data I had cleaned and used in my analysis? Should I change the variable names to make them more sensical to a human user? Or should I focus on machine readability and encode textual responses as numerical responses? Should I separate the qualitative data from the quantitative data? Should I embed the survey questions within the data?

${ }^{21}$ The Research Data Journal for the Humanities and Social Sciences is a collaboration between the publisher brill and DANS, a data repository. https://brill.com/view/journals/rdj/rdj-overview.xml ${ }^{22} \mathrm{https}$ ://datascience.codata.org 
A variety of guidelines and best practices have been developed for specific data types and within disciplines to help address similar questions (i.e. CESSDA, 2020). It took work, however, to locate appropriate guidelines, to sift through them and decide what pieces applied to my data and which were irrelevant. It also took work to follow up on the questions not addressed in these guidelines, by tracking down and consulting with data management experts. The onus ultimately lay with me, the researcher, to perform the mental gymnastics necessary to figure out which descriptions and practices were best suited to my particular data.

I ultimately decided to share the data used in the analysis presented in Chapter 5 and to rely on extensive data documentation to explain the cleaning process and the data themselves. Preparing the data for deposit and drafting this documentation - both a README file and the data paper - were not linear, disjointed processes, but occurred co-incidentally. If I made a change to the data, i.e. altering a variable name or correcting a spelling mistake, I also needed to modify the README file or change the tables which I had prepared for the data paper. The smallest change necessitated a string of alterations to ensure that all documentation and related files were in sync with each other.

Composing the README file itself was a welcome, straightforward process. To-the-point guidelines, such as those produced by the Cornell University Research Data Management Group (n.d.), helped to create a structured, succinct document describing the data collection, preparation and documentation, as well as to list references for related articles. If potential reusers had access to this README file and the related article (Chapter 5), I felt that they would be equipped to understand and use my data. What, then, would a data paper afford that a detailed, well-written README file did not?

\subsubsection{Affordances and duplication}

Potential benefits of data papers include providing space for extra contextual information, facilitating data discovery, indicating quality and enabling data citation. Some of these benefits are likely greater for data which do not have other associated publications. Both my methodology and potential biases were already detailed in the article presenting the survey results (Chapter 5); this article, which cites the shared survey data, is also already discoverable through search systems and will eventually be indexed in literature databases. 
The data paper does, however, include contextual information not presented in Chapter 5 or in the README file. The journal guidelines for the "usage notes" section in the data paper encourages authors to discuss critical data characteristics or to propose potential research questions for the data. This one-way communication between authors and data reusers may include its own set of biases. It can be difficult for data creators to imagine novel reuses for their own data (see Chapter 7). The questions which data authors propose may also be constructed to enable or obscure their own future research agendas, or to support analyses which have already been conducted.

The peer review of data papers in data journals has been suggested to provide an indication of data quality (Mayernik et al., 2015). The reviews which I received commented on the extent of the documentation presented in the paper, rather than on the data themselves. I had no indication if the reviewers had attempted to access or experiment with the actual data. The review processes for data papers with associated articles may also be different for those without. It is possible that other proxies for quality, i.e. the reputation of the journal where original results were published, may influence decisions made during data paper review.

Data papers may enable data citation, but they may also confuse it, particularly when other associated articles exist. It may be unclear to data reusers which publication to cite - the dataset, the data paper, the associated paper, or perhaps all three. In a world of metricladen research evaluation, this confusion will likely have repercussions for the calculation of citation counts and bibliometric indicators. This is also reminiscent of other practices which can be used to impact metrics, i.e. "salami-slicing," splitting research into the smallest possible publishable units, in order to increase publication counts.

Reporting related research in separate publications also makes it challenging to avoid selfplagiarism or text recycling. When writing the data paper, I spent significant time making small changes in wording or slightly modifying figures and tables to minimize possible duplication. The duplication of information and the variety of related publications requires more synthesis and harmonization work for potential data reusers. Individuals need to closely read and piece together contextual information distributed between metadata, data papers, README files and associated articles. This work is likely even more challenging when descriptions are very similar, as important differences may be harder to uncover. More information and publications may not always lead to better understanding. 
Duplicative work is also visible in the economic sphere. Authors and institutions often foot the bill for making data papers open access, for hosting data in a repository and for making related publications freely available. Editorial staff, publishers and referees also spend extra time, effort and money to review and prepare both data papers and related articles. Some of this duplication may be the result of applying formats and publishing practices standardized for journal articles to data papers.

\subsubsection{Standard formats}

Data journals aim for standardization. The majority require authors to use templates which, to a point, follow the overall structure of traditional journal articles, with sections for an abstract, background, methodology and references. Many also require a summary box with fields describing the data characteristics using controlled vocabularies, to be included at the beginning of the paper, i.e. SpringerNature's Scientific Data ${ }^{23}$ or Elsevier's Data in Brief ${ }^{24}$. This sort of standardization has potential benefits for machine readability and indexing, as well as for improving the readability of the paper. Such templates and descriptions may not be suitable for all data, however.

The section "technical validation" in this chapter, where authors are instructed to document experiments and analyses supporting the data's "technical quality," serves as a good example. This framing privileges one way of determining data quality, and also one way of describing it. Writing this section was an exercise in selling my data, using language which I would not normally use, in an attempt to prove their quality. This also made me question my own data. If I were unsuccessful in proving the (technical) quality of my data (that is, if the data paper was not published), what would it say about the quality of the already peerreviewed and published article reporting the results of my analysis?

The idea of "data journals" and "data papers" is itself adapting to a standard means of scholarly communication. Data journals are used to bring together data distributed in diverse repositories, using infrastructures built primarily for journal articles. The infrastructures required for article publishing, however, may not be the same infrastructures required to facilitate data reuse (Groth et al., 2020). There are also signs that the traditional format of the scientific journal may be changing, as journals, such as Quantitative Science Studies, ${ }^{25}$

\footnotetext{
${ }^{23} \mathrm{https}: / / \mathrm{www}$. nature.com/sdata

${ }^{24} \mathrm{https} / / / \mathrm{www}$.journals.elsevier.com/data-in-brief

${ }^{25} \mathrm{https}: / /$ www.mitpressjournals.org
} 
are experimenting with decoupling and outsourcing publishing and editing tasks and articles are increasingly posted on preprint servers.

\subsubsection{Closing}

It has been said that people tend to do what is easy, interesting or beneficial. Preparing, documenting and sharing data is not easy or straightforward, nor is it particular interesting. The process involves thorny choices and duplicative work, which may result in a cluttered landscape of scholarly artefacts, difficult for data reusers to navigate and understand. Data papers offer some benefits, particularly for data which do not have associated publications, but their overall benefit to data authors remains unclear. 
A dataset describing data discovery and reuse practices | 203 


\section{CHAPTER 7}

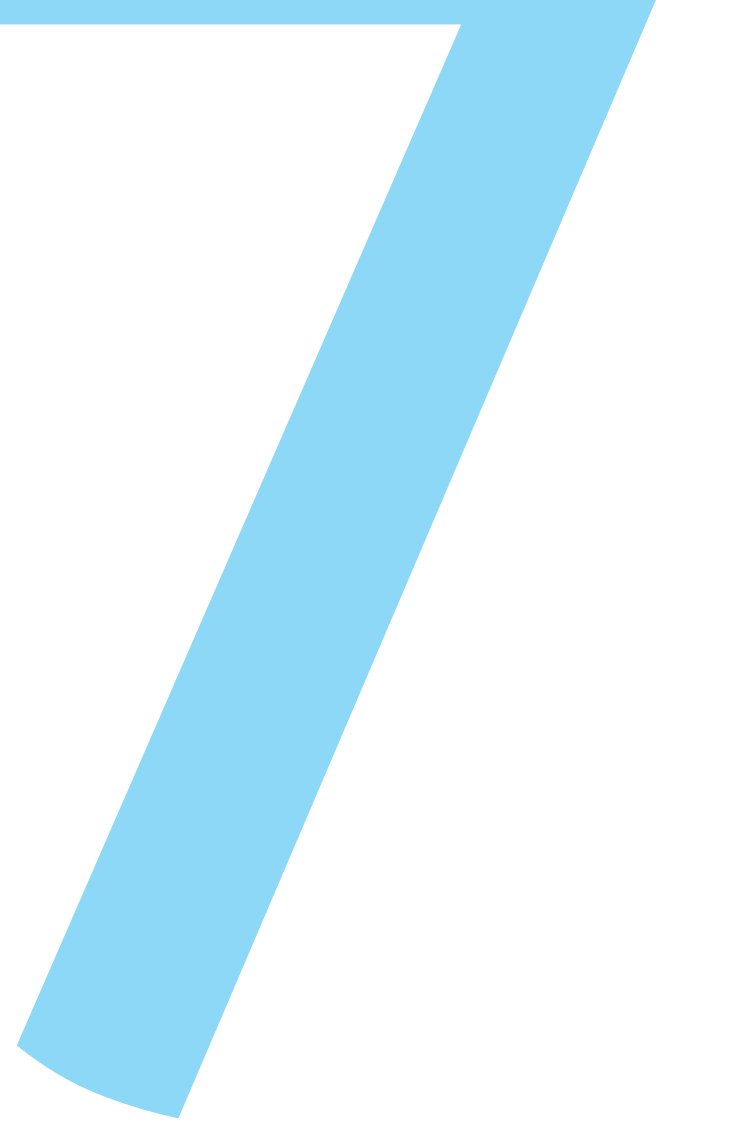




\section{Talking datasets: Understanding data sensemaking behaviors}

Originally published as: Koesten, L.*, Gregory, K.*, Groth, P., Simperl, E. (2020). Talking datasets: Understanding data sensemaking behaviors. International Journal of HumanComputer Studies. https://doi.org/10.1016/j.jhcs.2020.102562.

${ }^{*}$ Equal contributions. 


\title{
206 | Chapter 7
}

\begin{abstract}
The sharing and reuse of data are seen as critical to solving the most complex problems of today. Despite this potential, relatively little attention has been paid to a key step in data reuse: the behaviors involved in data-centric sensemaking. We aim to address this gap by presenting a mixed-methods study combining in-depth interviews, a think-aloud task and a screen recording analysis with 31 researchers from different disciplines as they summarized and interacted with both familiar and unfamiliar data. We use our findings to identify and detail common patterns of data-centric sensemaking across three clusters of activities that we present as a framework: inspecting data, engaging with content, and placing data within broader contexts. Additionally, we propose design recommendations for tools and documentation practices, which can be used to facilitate sensemaking and subsequent data reuse.
\end{abstract}




\section{1. Introduction}

Climate change; poverty; global hunger - all have been dubbed wicked problems (Peters, 2017 ) that have the best chance of being tackled by bringing together and using crossdisciplinary data in new ways (Walshe et al., 2020; CODATA, n.d.; United Nations, n.d.). Although data reuse is increasingly encouraged (European Commission, 2018c), it involves a host of challenges, such as providing rich, standardized metadata adequate for interoperability and reuse (Wilkinson et al., 2016). Fundamentally, reusing data also requires that data consumers make sense of data that others have created.

Even within their own disciplinary domains, understanding and making sense of data is a difficult and time- intensive process for researchers and data professionals (Kern \& Mathiak, 2015; Muller et al., 2019) which is heightened by the demands of navigating an increasing amount of digital information (Eppler \& Mengis, 2004). Also contributing to this difficulty is the fact that data do not speak for themselves, but require supporting structures - both social and technical - to convey the meaning necessary for reuse (Borgman, 2015a). The effort and costs involved in sensemaking can potentially be reduced through the development of automated tools and systems (Russell et al., 1993). Designing such tools is contingent upon first understanding and describing the behaviors involved in data-centric sensemaking Rogers et al. (2012).

Here, we identify and detail patterns of activities involved in data exploration and sensemaking. In the context of this work, data can be thought of as collections of related observations organized and formatted for a particular purpose, reflecting the variety of concepts different actors have of data, see (Borgman, 2015a). In order to identify these patterns, we draw on verbal summarizations as a method of uncovering sensemaking processes and build on the following ideas: (1) the act of summarizing is a form of sensemaking, (2) verbal summarization represents unique cognitive processes and (3) it is possible to identify common patterns in sensemaking activities when people describe data that they are familiar with and data that are unknown to them. We used these ideas to develop the following research questions:

- RQ1: What are common patterns of activities, both for known and for unknown data, in the initial phases of data-centric sensemaking?

- RQ2: How do patterns of data-centric sensemaking afford potential data reuse?

To explore these questions, we combined in-depth interviews with researchers, in which they performed a think-aloud task, and a screen recording analysis. During the interviews, 
researchers interacted with and verbally summarized an example of their own research data and a dataset that was unknown to them. We present the results from this study and use our findings to identify activity patterns and data attributes which are important across three clusters of sensemaking activities: inspecting data, engaging with data content more deeply and placing data within broader contexts. Finally, we detail design recommendations for tools and documentation practices to facilitate sensemaking and subsequent data reuse.

The key contributions of this work are identifying: (i) patterns of data-centric sensemaking activities; (ii) a framework for these activity patterns and their related data attributes; (iii) user needs for data reuse; and (iv) a set of design recommendations to support data-centric sensemaking.

\subsection{Background}

Sensemaking has been studied across a range of disciplines, including psychology (e.g. Klein et al., 2006), decision making (e.g. Klein et al., 1993; Malakis \& Kontogiannis, 2013), organizational behavior (e.g. see Maitlis \& Christianson, 2014 for a review), information seeking (Dervin, 1997; Marchionini \& White, 2007), and human computer interaction $(\mathrm{HCl})$ (e.g. Russell et al., 1993). In this work, we focus on sensemaking as discussed in information science and $\mathrm{HCl}$. In these domains, sensemaking is defined as the process of constructing meaning from information (Blandford \& Attfield, 2010), and is recognized as being an iterative process that involves linking different pieces of information into a single conceptual representation (Hearst, 2009; Russell, 2003).

\subsubsection{Sensemaking and information seeking}

Models of information seeking behavior often present sensemaking as a key component. Traditional models detail the specific steps involved in sensemaking during information seeking as a sequential, yet evolving, process (Sutcliffe \& Ennis, 1998; Hearst, 2009; Kuhlthau, 2004). While traditional models tend to be static, many of their authors emphasize that people's behavior is complex and changes when being presented with new information. More recent, dynamic models acknowledge a variety of influencing factors in finding and making sense of information, e.g. skills, knowledge, preconceptions, culture or motivation (Kelly, 2009; Klein et al., 2007). Other work examines the cognitive mechanisms involved, framing sensemaking as a series of different information processing components taking data as input and producing conceptual changes as an output (Bechtel, 2008; Zhang \& Soergel, 2020). 


\subsubsection{Data-centric sensemaking}

While sensemaking of textual information has been well-explored, there is a relative gap in research that aims to understand the strategies involved in making sense of data.

Compounding this is the fact that the very definition of "data", particularly "research data" has itself been the subject of much debate. An increasingly common conceptualization of research data is that proposed by Borgman (2015a): data are representations of observations, objects, or other entities that are used as evidence for the purposes of research or scholarship. This definition does not distinguish between data formats or qualitative or quantitative data, recognizing that what serves as data in one situation for one individual may not act as data in another situation for another individual (see also Pasquetto et al., 2017). Similarly, in their data frame theory, Klein et al. (2007) emphasize how the perspective (or frame) of the data consumer shapes the data in terms of how they are perceived, interpreted and even acquired. Through engaging with data, preexisting frames either change or get reinforced, which can be seen as an aspect of sensemaking. Critical data studies also describe this as a collective process, due to interpretative layers built into the creation and use of data (Neff et al., 2017)

Studies in $\mathrm{HCl}$ tend to focus on quantitative data, addressing, e.g., the role that visualization plays in identifying patterns in data (Furnas \& Russell, 2005; ah Kang \& Stasko, 2012); this focus reflects the emergence of bespoke visual exploration environments (Yalçin et al., 2018; Marchionini et al., 2005). Other work proposes tools to aid in sensemaking activities, such as a visual analytics system tailored for particular groups of data analysts (Stasko et al., 2008) or agile display mechanisms for users accessing government statistics (Marchionini et al., 2005). Investigations of exploratory data analysis (EDA) strategies, where new data are explored with a set series of procedures until a high-level story emerges, are also of relevance. Common EDA techniques include performing rough statistical checks and analyses (e.g. calculating descriptive statistics) or looking for general trends or outliers in the data (Baker et al., 2009; Marchionini, 2006). Many EDA techniques are graphical in nature and are undertaken to help assess the quality of the data.

The first phase of getting to know data, which can involve exploratory data analysis techniques, has been shown to involve a high level of cognitive effort (Zhang \& Soergel, 2014). Existing categories can prompt users to activate related memory content, resulting in converging categorization and verbalization processes; this influences how information is interpreted and potentially eases sensemaking efforts (Ley \& Seitlinger, 2015; Fiore et al., 2003). 
Engagement and sensemaking with data is also determined by the purpose of the engagement activity, usually connected to a task, which can range in specificity. The importance of quality indicators and uncertainty attached to data is task dependent (Boukhelifa et al., 2017; Koesten et al., 2017). While there are a variety of task classifications in the information seeking literature (e.g. Freund, 2013; Li \& Belkin, 2008), to this date there is no established taxonomy for data-centric work tasks, which might reflect rapidly changing work practices with data.

\subsubsection{Evaluating data for reuse}

There is a growing amount of literature, particularly within information science, that examines the reuse of research data. Key studies question and explore the definitions and types of data reuse within and across disciplines (Pasquetto et al., 2019; van de Sandt et al., 2019). Many studies characterize the contextual information required to make decisions about using (or not using) data within particular disciplinary fields (i.e. Faniel \& Yakel, 2017; Kriesberg et al., 2013). Although a set definition of context remains challenging (Faniel et al., 2019), there is an overall agreement that data reuse without any contextual reference is almost impossible to do well (Birnholtz \& Bietz, 2003; Borgman, 2015a).

Building on studies of researchers in three disciplinary domains, Faniel et al. (2019) propose a typology of the information needed to support data evaluation, finding that information about data production, data repositories, and data usage are key in making decisions about reusing data. Similarly, Gregory et al. (2020) find that researchers across disciplines rely on information about data collection conditions, data processing, topic relevance and accessibility when evaluating data. This aligns to a large degree with Koesten et al.'s findings on dataset-specific selection criteria covering different aspects of relevance, quality and usability (Koesten, Simperl, et al., 2019).

Other work looks specifically at how researchers develop trust in data. Yoon (2017a), for instance, draws on interviews with quantitative social scientists to explore the social, multistage processes involved in trust development. She identifies data characteristics which can aid in building trust, such as the quality of documentation and the reputation of the data publisher. Passi and Jackson (2018) describe perceived trustworthiness of data or a data science system as a task dependent and collaborative accomplishment that involves assessing different types of uncertainties. While the criteria used for both data evaluation and trust building likely play important roles in data-centric sensemaking, several authors highlight that much of the knowledge needed to make sense of data is tacit and not included in data documentation (Birnholtz \& Bietz, 2003; Rolland \& Lee, 2013). 


\subsubsection{Summarization as a way to understand sensemaking}

We adopt a study design that builds on work using summarization as a way of exploring cognitive processes (Hidi \& Anderson, 1986). Summarization tasks, as studied in psychology, are described as involving three distinct cognitive activities: selection of which aspects should be included in the summary; condensation of source material to higher-level ideas or more specific lower-level concepts; and transformation by integrating and combining ideas from the source (Hidi \& Anderson, 1986). As comprehension is viewed as a prerequisite for summarization, text summarization tasks have been used to assess recall and language abilities (Kintsch \& Van Dijk, 1978). We build on these ideas and use summarization as a way of exploring the cognitive processes involved in comprehending data as an information source.

In a recent study, Koesten et al. (Koesten, Simperl, et al., 2019) had participants produce written dataset summaries in order to better understand selection criteria for datasets. While these summaries provide insights into the conceptualization of datasets, written summaries do not always capture the complex verbal sensemaking that precedes their creation (Mayernik, 2011). Verbal, or spoken, summarizations often reflect deeper, more spontaneous cognitive processes (Crestani \& Du, 2006), but they have yet to be used to understand data sensemaking behaviors.

\subsubsection{Summary of key points}

We argue that in order to reuse data, data consumers must first be able to understand and make sense of those data. While we hypothesize that these sensemaking processes will include attributes similar to those identified in the above literature, we also postulate that data-centric sensemaking involves particular cognitive processes and social and technical interactions resulting in common patterns of sensemaking activities. Our work therefore takes into account not just attributes and categories related to engagement with data, but also considers the wider social, disciplinary and communication contexts existing in data work and their impact on consumer engagement with data.

Our argument builds on the assumption that sensemaking affords specific activities when engaging with data opposed to other information objects (e.g. textual sources), which is mirrored in the literature. However, the sensemaking and information seeking literature often either focuses on textual sources or does not clearly differentiate which source is addressed. 
It is also worth noting that there is a significant amount of literature on dataset reuse that focuses on operational problems, machine readability and data interoperability (Koesten et al., 2020). We do not review this literature in detail here, as our purpose is to focus on the less-studied practices and patterns involved in understanding data. We connect our work to these discussions regarding technical solutions for facilitating data reuse by providing empirical evidence of data-centric sensemaking and by identifying common patterns of sensemaking activities to enable design efforts.

\subsection{Methodology}

Our past work in textual data summarization (Koesten, Simperl, et al., 2019) and the reuse of research data (Gregory, Cousijn, et al., 2019) informed the creation of a semi-structured interview design examining how people verbally summarize and make sense of both familiar and unfamiliar data.

\subsubsection{Study design}

All participants were asked to bring data that they had used or were familiar with to share during the interview. We refer to this data as the known data in this paper. We left the decision about what constitutes "data" up to participants. The majority $(n=27)$ chose to bring data which they had created themselves. Most of the data brought by participants were spreadsheets ( $n=19$ ); other data included textual data (e.g. interview transcripts), images, videos or other artefacts. We did not ask for any documentation, supporting information or metadata from participants to see what they brought when not prompted.

We also prepared a dataset to share with participants; we refer to this data as the unknown data in this paper. This dataset was a modified version of a spreadsheet from a popular news source in the UK, the Guardian Data Blog, ${ }^{26}$ which was used in a previous study (Koesten, Simperl, et al., 2019). (See Figure 7.1; the entire spreadsheet is available on a GitHub repository ${ }^{27}$ associated with this work). This dataset met specific selection criteria: it included numerical and textual data, missing values, inconsistencies in formatting and some ambiguous variables. At the same time, the data were understandable and not specific to a particular domain.

\footnotetext{
${ }^{26} \mathrm{https}: / /$ www.theguardian.com

${ }^{27} \mathrm{https}$ //github.com/laurakoesten/talkingdatasets
} 


\begin{tabular}{|c|c|c|c|c|c|c|c|c|c|c|c|}
\hline & A & B & c & D & E & $\mathrm{F}$ & G & H & 1 & $\mathrm{~J}$ & k \\
\hline 1 & ID & Country & $\begin{array}{l}\text { Deaths, } \\
\text { confirmed } \\
\text { swine flu }\end{array}$ & $\begin{array}{l}\text { Deaths per } \\
\text { million } \\
\text { population }\end{array}$ & $\begin{array}{l}\text { Confirmed cases } \\
\text { (last updated } \\
\text { 26/01/2010) }\end{array}$ & $\begin{array}{l}\text { Infection rate } \\
\text { per million } \\
\text { people }\end{array}$ & LONG & LAT & $\begin{array}{l}\text { POP } \\
\text { (source: } \\
\text { UN) }\end{array}$ & $\begin{array}{l}\text { SOURCES (where no link, } \\
\text { based on agency reports) }\end{array}$ & $\begin{array}{l}\text { Second source, if } \\
\text { needed }\end{array}$ \\
\hline 2 & & Afghanistan & 17 & 0.6 & 837 & 29.73 & 65 & 33 & 28150000 & ntrp://mwwe amro who int/osr/h1n $1 / \mathrm{m}$ & In1 update htm \\
\hline 3 & & Albania & 6 & 1.89 & 13 & 4.1 & 20 & 41 & 3169000 & Ittpi/lecdc.europa.eu/en/activities/s & urveillance/EISN/Pages/EISN \\
\hline 4 & & Algeria & 57 & 1.63 & 470 & 13.64 & 3 & 28 & 34895000 & hitp://www. afro. who.intdddcinfluenz & e http://www.ands.dz/grippe-pe \\
\hline 5 & & Andorra & & 0 & 1 & 11.63 & 1.5 & 42.5 & 86000 & hittp://wwww.who int/csr/don/2009 07 & 27/en/index.html \\
\hline 6 & & Angola & 0 & & 37 & 2 & 18.5 & -12.6 & 18498000 & http://www.afro, who.intdddolinfluenz & :aa/updates/ \\
\hline 7 & & Antigua and Barbuda & 0 & 0 & 4 & 45.45 & -61.8 & 17.05 & 88000 & http://how.paho.org/ho/index.php?o & ption $=$ com contentstask $=$ blog \\
\hline 8 & & Armenia & 3 & 0.97 & & & & & 3090000 & ntrp:/lecdce europa eulen/activities/s & Urveillance/EISN/Pages/EISN \\
\hline 9 & & Australla & 191 & 8.97 & 37553 & 1.763 .63 & $-2 T$ & 133 & 21293000 & http://lecdc.europa.eu/en/activities/s & Urvelllance/EISN/Pages/EISN \\
\hline 10 & & Austria ${ }^{*}$ & 0 & 0 & 361 & 43.16 & 47.3333 & 13.3333 & 8364000 & http:l/lecdc.europa.eu/en/activities/s & Urveillance/EISN/Pages/EISN \\
\hline 11 & & Argentina & 626 & 15.54 & 10209 & 253.48 & -34 & -64 & 40276000 & http://new.paho.org/ho/index.php?o & otion $=$ com contentstask $=b l o g$ \\
\hline 12 & & Azerbajan & 2 & 0.22 & 2 & 0.22 & 47.5 & 40.5 & 8934000 & http://mwwwwho intoerldon/200908 08 & 04/onlindex.htm! \\
\hline 13 & & Bahamas & 4 & 11.7 & 24 & 70.18 & -76 & 24 & 342000 & http://new.paho.org/ho/index.php?o & ption $=$ com contentstask=blog \\
\hline
\end{tabular}

Figure 7.1: Excerpt of the provided or "unknown" dataset describing the global occurrence and mortality rate of swine flu

\subsubsection{Data collection}

The interview protocol (available on GitHub) consisted of two primary sections: questions about the known data, and questions about the unknown data. Interviews lasted $30-60$ minutes and were held using the web conferencing application Zoom. All interviews were audio-recorded; screen recordings capturing participants' interactions with the data were obtained for 26 interviews. (Five recordings were not created due to technical problems). Both sections of the interview began with the verbal summarization task. The task was for participants to provide a general summary description of the data to someone who is trying to decide whether to use the data, but who is unable to see it. We formulated the task in this fashion in order to elicit rich descriptions of the data, not aimed at a particular use case.

In the first section of the interview, after summarizing the known data, participants were asked questions about data reuse and their data creation and documentation practices. In the second section of the interview, we shared our dataset and asked participants to perform the same verbal summarization task, this time on the unknown data. We then asked them to describe and discuss specific areas of the unknown dataset and posed follow-up questions about data reuse, data sharing and data search (examples can be seen in Table 7.1). 


\begin{tabular}{|c|c|}
\hline Section & Examples \\
\hline 0 : Background & $\begin{array}{l}\text { Demographics, job role, discipline or research area, experience of } \\
\text { working with data }\end{array}$ \\
\hline 1: Summary of known data & Verbal summarisation task \\
\hline \multirow[t]{2}{*}{ 1: Context of known data } & Information structures needed for reuse \\
\hline & Describe for colleagues vs someone outside your domain \\
\hline 2: Summary of unknown data & Verbal summarisation task \\
\hline 2: Context of unknown data & Anything missing that you would like to know about the dataset \\
\hline 3: Specific areas of unknown data & $\begin{array}{l}\text { Rows / columns with missing / ambiguous data, different variable } \\
\text { types }\end{array}$ \\
\hline
\end{tabular}

Table 7.1: Overview of interview schedule including the summarization task and example topics for the different interview sections

Two pilot interviews were conducted in October 2018. This allowed us to determine the interview duration, to finetune the interview questions and the set-up of the summarization task. The remaining interviews were held between November 2018 and January 2019 and were transcribed by a professional transcription firm.

\section{Recruitment}

Our primary sample was drawn from a pool of individuals, past respondents to a large scale survey study conducted by (Gregory et al., 2020), who had published at least one article indexed in Elsevier's Scopus literature database ${ }^{28}$ in the last three years.

We sent recruitment emails $(n=1000)$ in November-December 2018 in two batches and received 47 positive responses. From those, we selected 27 participants who represented a range of disciplines and nationalities and were proficient in English. We recruited an additional four participants via convenience and purposive sampling, for a total of 31 participants. Participants for our pilot interviews were identified using purposive sampling. We did not offer incentives for participation in this study.

\section{Participants}

Participants ranged from age 26 to age 73 , with the majority being between 30 and 45 years old (Median 40.6). They reported 19 different countries of residence worldwide, with a skew towards the Netherlands $(n=5)$ and the UK/USA $(n=3) .13$ out of the 19 countries are in Europe; 20 of our 31 participants live in European countries.

Although participants work in multiple countries, the majority were fluent in English; minor problems with language or internet connectivity were experienced in two of the interviews.

${ }^{28}$ https://www.scopus.com 
Over half of the participants $(n=18)$ worked at a university or college at the time of the study, with six working in research institutions. Participants' disciplinary domains and roles are described in Table 7.2. All participants have previously published research papers. The majority were experienced with quantitative research; others categorized themselves as predominantly qualitative researchers, or used both quantitative and qualitative methods.

\begin{tabular}{cll}
\hline $\mathbf{P}$ & Domain & Role \\
\hline 1 & Biological sciences & Project manager \\
2 & Life sciences, Paleontology & Project acquisition manager \\
3 & Biblical studies, Information Technology & Researcher \\
4 & Musicology, Humanities & Project leader, project manager \\
5 & Geophysics & Data curator \\
6 & Physics, Chemistry & Post doctoral associate \\
7 & Analytical Chemistry & Researcher \\
8 & Material Science and Engineering & Professor emeritus, researcher \\
9 & Social Science (Social Care, Social Work) & Senior research fellow \\
10 & Social sciences, Computer science & Director of Research Services \\
11 & Social justice, Socioeconomic Justice & Professor \\
12 & Geology, Earth Sciences & Research scientist \\
13 & Earth Sciences & PhD student \\
14 & Fluid Mechanics & Researcher \\
15 & Molecular Biology & Researcher \\
16 & Tourism, Social Psychology & Senior lecturer \\
17 & Mathematical education & Assistant professor \\
18 & Telecommunications, Computer science & Associate professor \\
19 & Biological anthropology & Postdoctoral research fellow \\
20 & Medicine, Biomedicine & Researcher and teacher \\
21 & Agriculture, Food science & PhD Student \\
22 & Medicine & Surgeon, PhD student \\
23 & Entomology (Biological Sciences) & Researcher, curator \\
24 & Environmental sciences, agriculture & Lecturer \\
25 & Biostatistics, Epidemiology & Associate professor, biostatistician \\
26 & Material Science & Researcher \\
27 & Psychology & Researcher, PhD student \\
28 & Veterinarian, Obstetric Clinician & Assistant professor \\
29 & Information science, Medicine & Associate director \\
30 & Environmental sciences & Researcher \\
31 & Medicine, Mental Health & Head of research group in a hospital \\
\hline & & \\
\hline
\end{tabular}

Table 7.2: Description of participants $(\mathrm{P})$ with their disciplinary domains and professional roles

\section{Ethics}

The study was approved by the University of Southampton's Ethical Advisory Committee under ERGO Number 45874. Informed written consent was given by the participants prior to the interview. 


\subsubsection{Data analysis}

\section{Coding strategy}

The coding strategy for thematic analysis was developed through a multi-step process of independent parallel coding (Thomas, 2006), using the qualitative data analysis program NVivo. Two authors independently analyzed a sample of seven interview transcripts and developed an initial codebook with supporting examples, employing a combination of deductive and inductive thematic analysis (Robson \& McCartan, 2016). Codes developed through deductive analysis were oriented on the different sections of the interview protocol and on existing literature in data summarization (Koesten, Simperl, et al., 2019) and data reuse (Faniel et al., 2019; Faniel \& Yakel, 2017).

Within these high-level themes, the authors iteratively developed codes based on a general inductive approach (Thomas, 2006) through sequential readings of the transcripts. The independently developed codebooks were compared for similarities and differences and combined and modified to create a single unified codebook which was then used to re-code the sample transcripts, which were divided evenly between the two researchers. To further enhance the reliability of the coding scheme, two senior researchers checked and discussed the unified codebook for a sample of the data.

Based on this analysis, we made further modifications, resulting in a nested coding tree consisting of three primary codes with a total of 30 child codes (see Figure 7.2 for the most used codes). We consolidated these codes through axial coding (Straus \& Corbin, 1990) drawing out those links which allowed us to answer our research questions. The themes identified through axial coding are used to structure the Findings section and form the basis of the synthesis presented in Figure 7.7. 


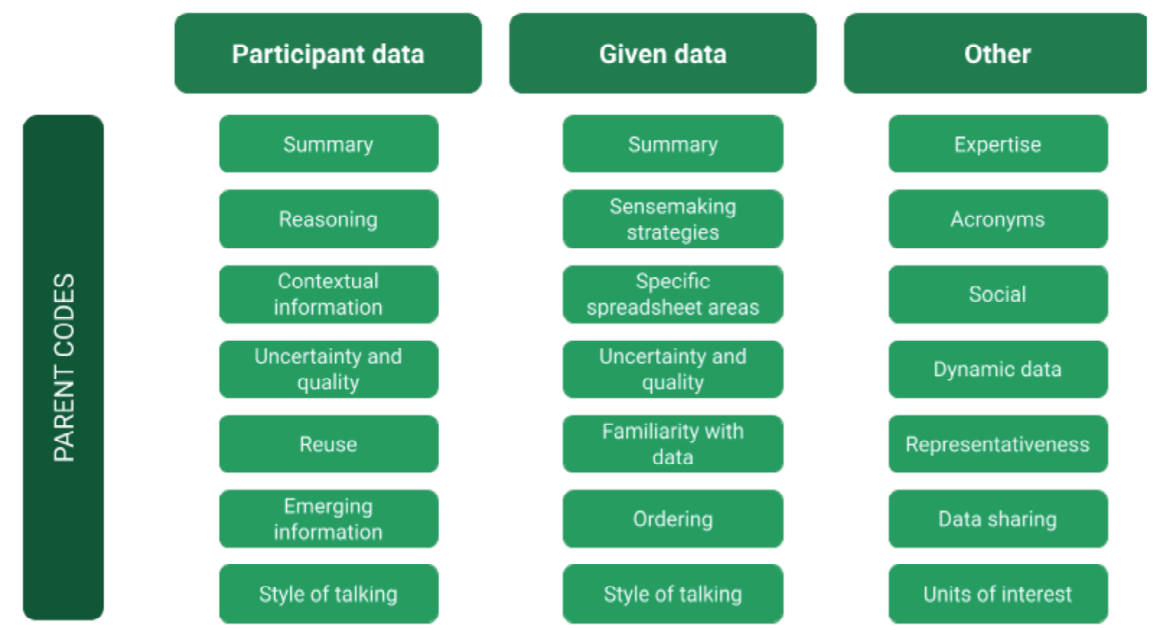

Figure 7.2: Primary codes

\section{Screen recording analysis}

We analyzed the 26 captured screen recordings to identify common interactions with the unknown dataset. We examined participants' actions during the general summarization task. We did not analyze the screen recordings of the summarization task for the known data, due to the diversity of participants' data, participants' privacy concerns and in order to encourage deeper descriptions of their data. Two of the authors independently viewed a sample of these screen recordings to identify common interactions. The authors then discussed the list of interactions and iteratively developed a list of 15 interactions to use in the video analysis. We used this list to identify the first occurrence of each possible action; we did not consider the duration of each action in our analysis. The coding of the screen recordings was done by one researcher only. Even then, a sample of the coded data was checked by the other researcher. Via the screen recordings, we could document how much of the spreadsheet participants could visibly access on their screens without scrolling. This allowed us to control for larger screens.

\section{Visual analysis}

All plots were created using the statistical analysis program R. We used the color palette "viridis", as it has been shown to be more accessible than other comparable color schemes (Rudis et al., 2018). 


\subsection{Findings}

We present our results along two dimensions: the research questions identified in the introduction section and the clusters of sensemaking activities which we identified via axial coding, namely inspecting the data, engaging with the content and placing data in broader contexts. Although we divide this section by research question to improve readability, the evidence we present often spans these divisions.

We pay special attention to both activity patterns, which we define as common physical and cognitive actions undertaken by participants when engaging with the data, and data attributes, or characteristics of the data with which participants interacted. We examine the findings in light of data reuse and synthesize them in the Discussion section to provide an overview of the patterns we identify.

\subsubsection{RQ1: What are common patterns in sensemaking activities, both for known and for unknown data, in the initial phases of data-centric sensemaking?}

\section{Inspecting}

When participants were first shown our dataset, we asked them to perform the verbal summarization task - to provide a general summary description, after taking a few minutes to explore the data silently. In this section, we examine both the order of how participants discussed attributes of the data (see Figure 7.3 and 7.4) and their actions in the spreadsheet during these verbal summarizations (Figure 7.5).

\section{Order of verbal summarization.}

We observed two approaches when completing the verbal summarization task: participants took either a linear or an interwoven approach. In linear summaries of the unknown data $(n=$ 24), participants addressed the data attributes identified in Figure 7.3 (e.g. time, location, format) one-by-one before proceeding to the next attribute. In the interwoven summaries, participants interspersed descriptions of individual attributes with their analyses and comments $(n=7)$. 


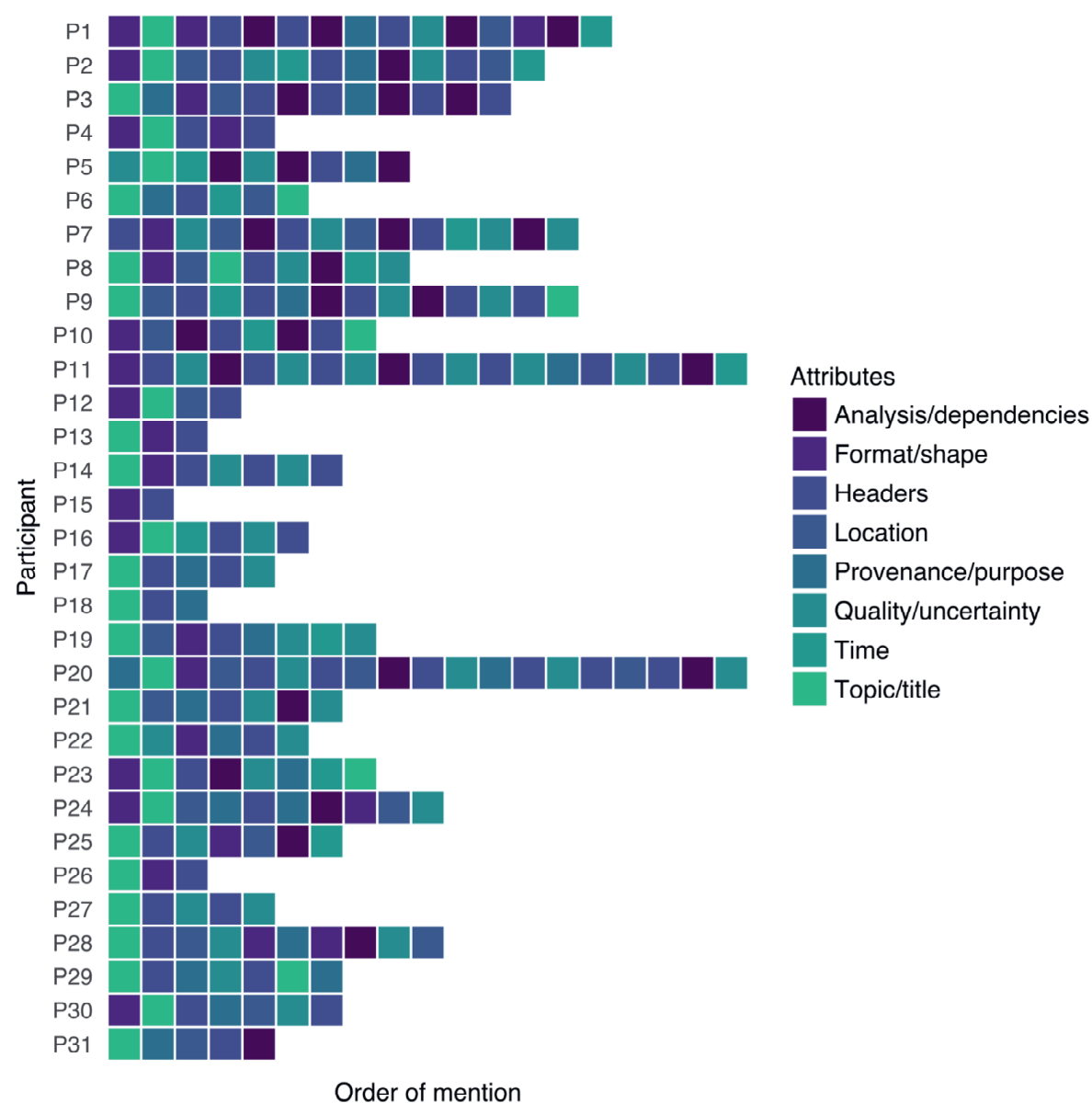

Figure 7.3: The order in which participants discussed certain attributes in the unknown dataset

Figure 7.3 shows the attributes headers $(n=64)$, quality/uncertainty $(n=42)$, topic/title $(n=$ $33)$, and analysis/dependencies $(n=30)$ were most frequently mentioned in the summarization of the unknown dataset. The majority of participants mentioned the overall topic or title as one of the first two attributes $(n=24)$; roughly half of participants mentioned the format or shape of the data (e.g. the number of columns, rows or observations) either first or second $(n=15)$. The discussions of other attributes were likely influenced by the structure of the dataset itself. Location information was a prominent part of the dataset, e.g., as the data were ordered by country and the four columns containing geographic information were positioned on both the left and right sides of the spreadsheet. The data included only minimal temporal information. The majority of general summaries mentioned location $(n=$ 
22) toward either the beginning or end of the description, while temporal information was mentioned in just under half of the general summaries $(n=13)$.

In the linear general summaries, time and location were discussed or questioned at a general level:

This communication shows us the deaths from swine flu in the countries around the world, Afghanistan, Albania, Columbia, Bolivia. (P31)

The one thing that is not apparent immediately from the data is the time span. (P19)

Participants taking an interwoven approach to summarization engaged in more initial analysis, repeatedly seeking relationships and dependencies between the spreadsheet columns or expressing uncertainty about meaning or the quality of the dataset.

I don't see any date or year, for purposes of comparison then it's a bit problematic, I can for example only do comparison charts for those with an asterisk for Austria and Bulgaria, for example, because they all have the data from 2009 but for number of deaths recorded in that country, then this data is useful, infection rate per population. (P5)

We observed similar attributes within the general summaries of participants' own data, but participants also mentioned additional attributes, i.e. details of their own field of research, methodology and details of the particular study, data availability and access restrictions, and the existence of additional information or documents needed to describe and understand their data (Figure 7.4). 


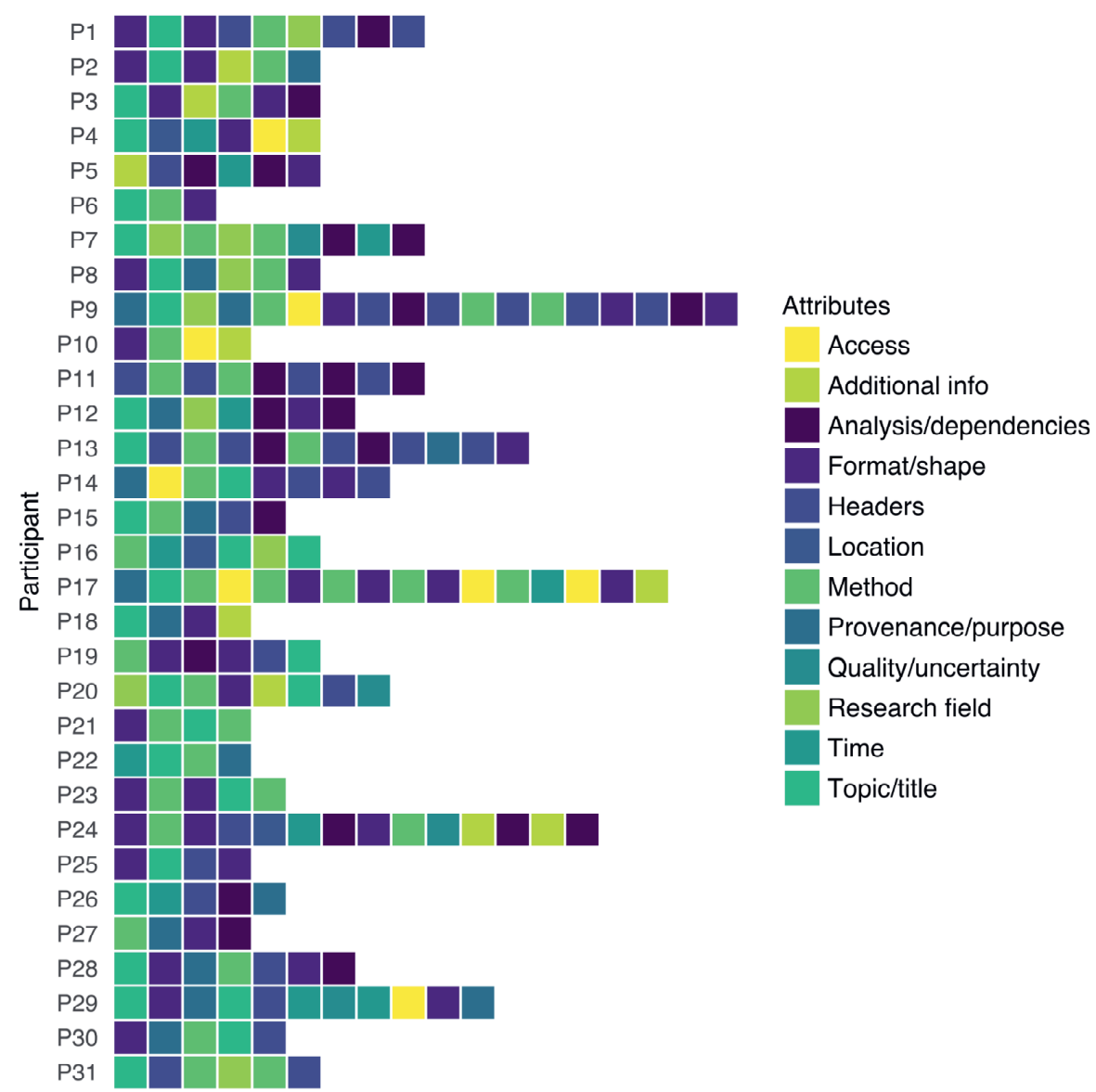

Order of mention

Figure 7.4: The order in which participants discussed certain attributes in their own data (the known data).

Most of the general summaries of participants' data followed a linear pattern $(n=24)$. This could be because participants were not working to understand their own data, but were rather aiming to make their data understood. They were also to some extent better prepared for the requirements of the task, having already had experience working with and discussing their data. In interwoven summaries of their data $(n=7)$, participants mixed descriptions of study methodologies with descriptions of headers and data format; some relied on methodological descriptions to communicate the general topic of their data.

These are experiments from a 50 meter long indoor set up that we have, where we ran gas and oil through the pipeline, through a 60 meter long pipeline, and we 
measured the average values - so pressure drop and build-up. And we did that for different gas and liquid velocities, and they also changed the type of oil, so we did this with one oil with a quite low viscosity and one with oil with a quite high viscosity. (P14)

\section{Actions in the spreadsheet.}

The actions captured in the screen recordings of the verbal summarization task for the unknown data support the attributes identified in Figure 7.3 and 7.4. Figure 7.5 shows the total number of actions observed, as well as the frequency of their order of occurrence. Scrolling right $(n=24)$ was the most frequently observed action, followed by scrolling down $(n=23)$. Participants also clicked on or indicated column headers and specific values. Clicking on both headers $(n=18)$ and particular cells $(n=17)$ occurred more often than other forms of indicating these areas of the spreadsheet. Four participants indicated headers in other ways, i.e. hovering over or circling them, yielding a total of 22 participants who either clicked on or indicated headers. Four participants also pointed out particular cell values using these alternative actions, resulting in a total of 21 participants who either clicked on or indicated cell values.

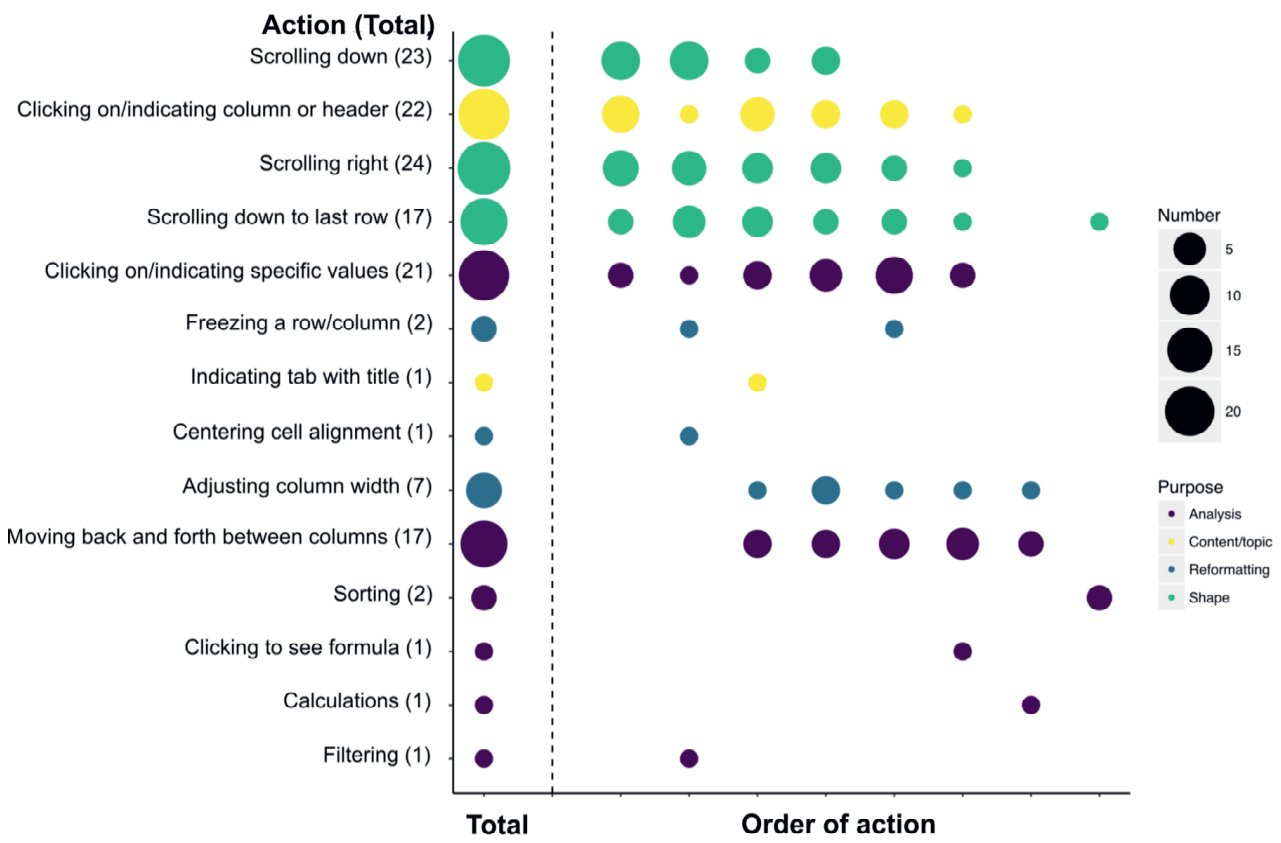

Figure 7.5: Total number of actions and order of actions observed in screen recordings of the unknown dataset. Size of circle represents number of participants engaging in activity. Figure is arranged according to which activity most frequently occurred first. Color represents purpose of action. 
Analyzing the order of these actions show that the majority of participants began by determining the length, breadth and general topic of the unknown dataset. Nine participants first scrolled down, while eight clicked on headers and seven initially scrolled to the far right of the spreadsheet. Once participants established the general shape of the data, more analysis-related actions were observed, most noticeably examining specific cell values by clicking or indicating and moving back and forth between different columns. One example of left and right scrolling was switching between the different types of geospatial columns which were not located in close proximity to one another.

Some participants prepared for analysis by reformatting the spreadsheet $(n=10)$, i.e. by adjusting the column width or freezing columns or rows. (For three recordings, the width of one of the columns was not optimized to allow reading one of the header names). Analysis features of the spreadsheet were only used in four instances, for actions such as sorting, filtering, or performing calculations. This reflects the nature of the think aloud task and the time limitations of our study.

We began examining screen recordings for participants' data after the general summarization task. These screen recordings provide a different type of insight, revealing actions that participants took to ensure that the interviewer adequately understood their data. The actions that we observed ranged in complexity. Participants with spreadsheet data often clicked on each column header, as they provided more detail about each column. Others demonstrated how they would analyze the data, showing unique functions of their analysis software or creating sample spreadsheets and plots.

\section{Engaging with the content}

Participants engaged with data content in more depth as they worked to explain and understand the data. This stage of deeper interrogation sometimes began during the scanning phase; it occurred both when interacting with the unknown dataset and with the known data. Table 7.3, which is further discussed throughout this section, presents quotations demonstrating similarities and differences in how they engaged with both known and unknown data. This table is organized along three themes: encodings, or codes developed to understand and interact with data, acronyms and abbreviations and identifying "strange things" within the data. 


\begin{tabular}{l}
\hline Known data \\
\hline Encodings \\
(P23): Because of the way it's set up, while it may appear on \\
screen in words, it's actually all in zero, one, two, three, four \\
up to nine. But you can present it yourself in words, and that's \\
really helpful if you're scoring, be- cause you can actually \\
click from one cell to the next, and down the base it will \\
actually tell you what that char- acter is in that cell and \\
whether you just code a zero or a one. \\
(P9): Age band and then I grouped the age bands into adult \\
and older people, and that was one of the issues of each of \\
our journeys, a different way of categorising people, so I \\
ended up with a very broad age range really.
\end{tabular}

Acronyms and abbreviations

Identifiying "strange things"
(P22): That is a classic abbreviation in the field of hepatic surgery. AFP is alpha feto protein. It is a marker. It's very well known by everybody...the AFP score is a criterion for liver trans plantation.

(P28): So if there is strange code that people cannot understand, I make a legend. Normally the colleagues I'm working with, we use our terms, so I tend to use the most user terms like LD; like SEM is typical for...everyone in my field.

(P7): Let's say from previous experiments and or runs, you know that repeating the experiment, you would get within an error of say $5 \%$ or $2 \%$, whatever the case maybe. So obviously these three [indicating error bars] are huge, and it would mean that you will have to repeat. So either some- thing is wrong in your system, or you get something wrong during the sample preparation, or the system's not stable, or something else is going on. Or that you're just not planning enough repetitions to get to the true value, so I think it is an important measure to determine if you've got reliable data.
Unknown data

(P28): I don't know if it could be interesting to be banded in categories like, I don't know, continents... it depends.

(P19): It looks like they started to code for if there are no deaths, then it's coded as a zero, but there are some instances where there are missing data.
(P20): If I would make this assumption, I would say this is like geographical location of the countries, but I have no idea what is 'Long' and 'Lat'. In my work, I have never encountered these kind of acronyms, so it's currently hard for me to assume what would this mean in the context of swine flu.

(P7): I'm not sure what 'long' means. I wonder if it's not something to do with longevity. On the other hand, no, it's got negative numbers. I can't make sense of this.

(P20): If I would not go into those cases, like with these discrepancies. I would just assume that this column indicates only the optimised data about whether they're aware or they're not, that [deaths are] due to swine flu in these countries."

(P14): That is simply a column saying if there are any deaths at all or not for a certain country related to the swine flu. I see there is a formula here, just simply checking if Column $L$ is larger than zero. So exactly using this information...so then that means there is something wrong with the formula or I completely misunderstood what Column $L$ is.

Table 7.3: Exemplary quotes illustrating participants' interactions with both their own and the unknown data.

In this phase, participants identified patterns and trends (e.g., via simple analyses or discovering relationships between columns) and discussed encodings, often related to categorizations, expressed within the data. They also explored uncertainties attached to the data and the data's overall integrity. In addition to further discussing these approaches, we point out two other particular instances observed in this level of engagement with the data: understanding strange things and collaborative sensemaking.

\section{Data analysis, encodings and tools.}

When discussing their data, participants demonstrated how they seek patterns and relationships by creating plots, switching between layers on geospatial images, and developing scales and formulas. Participants also expressed a desire to create plots to visualize the unknown data to identify trends and sought anchor variables as they investigated individual columns and described sample rows. They further drew attention to 
columns with limited value ranges in their summary descriptions, e.g. columns with binary variables or those with only a few categorical variables; fewer analyzed the range of values in columns with continuous variables.

Participants "encode" their own data in ways that help them more easily identify trends and generate findings by, e.g., converting categorical variables to numerical values and vice versa. These encodings are often influenced by the specifications of the analysis tools and software which participants use, such as SPSS, R, or domain-specific programs; which can also influence how participants structure their data, at times increasing the data's machine readability.

I use this data to create variables in SPSS. The one I'm looking at now has still got all the labels as words; I thought it would be easier to look at as a spreadsheet. There's another process I went through to translate the words into numbers. For SPSS, you really need numbers in the value labels. That was a whole process, to go through of coding the written, the categories, but just adapting those into numbers that I use. (P9)

[We are] working in $\mathrm{R}$ and our supervisor wrote a package which can easily work on it, but the main aspect is that you have to have grouping variables and independent variables which are the sensor signs. Then you have to separate the data to these different types, so the grouping variables and the independent variables because the $\mathrm{PC}$ and the IDA in the R can work in this structure. (P21)

Other forms of encoding included developing broad categories or groupings to describe and analyze data, such as differentiating between raw data and derived data, or numerical and non-numerical data. Participants also created groups of certain columns according to their semantic meaning; demographic variables were mentioned together, as were descriptive attributes for the same instance, e.g. "columns with sources" or "socioeconomic measures". These types of encodings were observed when participants discussed both their own and the unknown data. When working with our data, participants also searched for how null values were encoded and represented (see Table 7.3).

While the majority of participants reported using spreadsheets or Microsoft Excel at some point in their data workflows, very few actually made use of the built-in analysis tools in our spreadsheet at any time during the task. This could be due to time limitations during the interviews or to the fact that participants were not familiar with the Google Sheets environment which we used. It could also be a result of the fact that some participants do not 
use spreadsheets to analyze data directly, but rather reported using them for other purposes, such as recording and organizing data or cleaning and preparing data for analysis. Spreadsheets are also used by participants to specifically enable sharing data in a way that is easily accessible or compatible with a variety of analysis programs, facilitating data reuse.

Expressing uncertainty, seeking quality and understanding strange things.

Both when discussing their own data as well as when engaging with our data, they expressed concerns about potential misinterpretations, focusing on questions that could arise due to misunderstandings about how data were cleaned and processed. For both quantitative and qualitative data, participants viewed the encodings and categories that they had constructed as major risk points for correctly interpreting their data. The encodings that facilitated their own use of the data (as a data producer) may not be helpful or be explained well enough to enable appropriate data reuse by potential consumers of their data.

Although we've tried really hard, because we've put in a coding frame and how we manipulate all the data, I'm sure that there are things in there which we haven't recorded in terms of, well, what exactly does this mean? I hope we've covered it all but l'm sure we haven't. (P10)

They also questioned and critiqued the meaning of the known data, highlighting the lack of contextual information about how the dataset was created and the use of unexplained abbreviations in the dataset. When discussing their own data, however, participants often referred to unexplained acronyms or abbreviations common in their own disciplinary domains (see Table 7.3).

Participants combined their interpretations about the meaning of our dataset along with analyses of its completeness and how missing values are reported to make quality determinations. They also used missing values as checkpoints to identify relationships between columns and to identify potential errors or anomalies in the data.

So the data is fairly complete with really limited missing values, so the quality of data looks good. (P29)

It's got some blanks, which I presume means no data has been given. Although that's interesting...there's some missing data which shouldn't be missing. Because Armenia, for number seven say, it reports three deaths and yet the swine flu deaths is blank, so that's a bit of an anomaly, and there are quite a few blanks actually. (P9) 
Participants looked for other unexpected values (e.g. outliers) or inconsistencies in formatting or standard ways of reporting to assess the precision and accuracy of the unknown dataset. Wrestling with these strange things often served as the entry point to a deeper engagement and understanding of the data, allowing participants to question their assumptions and initial understandings (see Table 7.3).

Now that sounds quite high for the Falklands. I wouldn't have thought the population was all that great...and yet it's only one confirmed case. Okay [laughs]. So yes...one might need to actually examine that a little bit more carefully, because the population of the Falklands doesn't reach a million, so therefore you end up with this huge number of deaths per million population [laughs], but only one case and one death. (P23)

Some of them have decimals, like a lot of decimals, and some don't have any decimals. So I don't know whether that means that those are supposed to be measured more precisely...or that there is an inconsistency of using the amount of decimals per cell. (P1)

Encountering the unexpected in their own data is a critical and normal part of participants' research processes (see Table 7.3). While anomalies can be indicative of possible mistakes or points for improvement in the study design, they can also reflect unexpected external changes to the study environment, e.g. people withdrawing from a study, or new technologies that have been adopted over the course of long-running studies. Participants repeatedly emphasized the need to communicate information about these changes or potential sources of error to possible data reusers:

When I'm explaining the dataset by sharing a screen or showing them the file or to someone who would probably understand the data, from a dataset perspective, I would basically talk about the implausible values and the missing values and if I'm aware of the issues related to the data, I would like to point them out. (P29)

\section{Sensemaking through "collaborations".}

Working with team members is key to making decisions about study design and analysis, i.e. deciding which data are important to record and analyze, how to develop scales, clarifying study details and making sense of mistakes or unexpected values in the data. 
I know roughly what it consists of, but I didn't know precisely, and I had to go back to the person who generated it and say "What does column D mean? And where is the location of the thermocouple whose temperature is measured in column E?". (P8)

We have a table with...almost 30 columns with variables that were collected, including the names of the people who went into the field and collected each of the samples. So we are keeping track of who's responsible for each of the samples, then if we find any error, any mistake, then we can contact those people. (P24)

During the interviews, participants also collaborated with the interviewer to ensure that the interviewer correctly understood their data. Often, important details crucial to understanding the data emerged only when both the interviewer and the participant could see and interact with the data together. We saw this, e.g., in the case of learning about the importance of temporal information in coral reef imaging data or highlighting a key variable (inflammation) in a study about bipolar individuals. For some, it was nearly impossible to explain their data without being able to indicate specific areas of an image or demonstrating how error analysis was conducted.

\section{Placing}

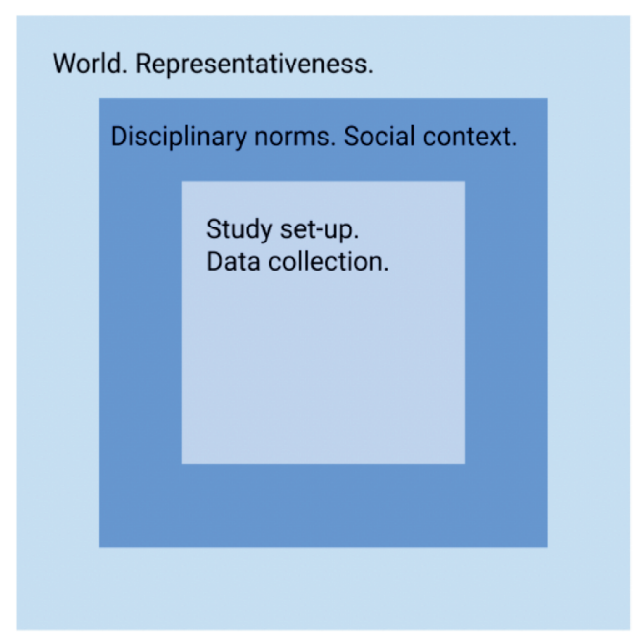

Figure 7.6. Placing data in contexts

As they engaged more deeply with data, participants placed data into existing contexts, practices and knowledges; this process of "placing" occurred at different scales (Figure 7.6). Data were placed within their immediate contexts of creation, e.g., when participants detailed 
study designs, experimental setups or the conditions surrounding data collection, including broader temporal or geographic details.

And it describes, or rather it comprises the results of a laboratory experiment lasting about an hour in which the experimenter,[..], is inducing the crystallization of a salt in a porous rock sample. And as the crystallization proceeds, two things happen. One is that the heat is evolved and so the temperature changes and the rock sample slightly increases in temperature, and we measure that temperature at three different positions. In addition there is a very slight expansion of the rock which we detect from the output of a very sensitive mechanical gauge. And then these four measurements, the three temperatures and the mechanical strain are measured at intervals of one second over a period of a few hours and so the dataset consists of this set of numbers. (P8)

These types of contextual details have the potential to impact the meaning which the values themselves carry.

Error bars depend a lot on the experimental conditions and on the condition of the material. So, for example, if it was used on powder samples then the error bars would be bigger than the ones that were obtained on single crystal data. (P6)

At a broader level, participants conceptually placed data within the norms of their disciplinary domains, referencing discipline-specific methodologies and limitations, ways of analyzing and verifying data or common data formats. They also recognized that broader social contexts can influence the sensemaking process.

Finally, participants attempted to place data within the world, gauging how representative data are of a particular phenomenon. These judgements reflect assumptions about how much the data reflect reality, as data themselves are usually samples which are hardly ever complete, unbiased or without conflict or ambiguity.

It's a pretty large sample size, again, 1, 260. We have equal numbers of males and females. We have three ethnicities: Caucasian, African American and Hispanic, equal numbers of each of those. So it's a well-balanced data set and, because of that, if you were to be interested in how these different cultural values vary or not based on ethnicity, it would be an excellent dataset. (P19) 
One simple example we observed in our dataset was a contention about the representativeness of the countries, which showed a range of interpretations and was expressed in a variety of ways. Participants questioned both the completeness of the list of countries and also whether the data represented the entirety of each country.

P2: It's listing the countries for which data are available, not sure if this is truly all countries we know of...

P8: It includes essentially every country in the world

P29: Global data

P30: I would like to know whether it's complete...it says 212 rows representing countries, whether I have data from all countries or only from $25 \%$ or something because then it's not really representative.

P7: If it was the whole country that was affected or not, affecting the northern part, the western, eastern, southern parts

P24: Was it sampled and then estimated for the whole country? Or is it the exact number of deaths that were got from hospitals and health agencies, for example? So is it a census or is it an estimate?

During placing activities, participants commonly reported the need to know the original purpose for which the data was created. Descriptions of their own data's original purpose were often complex, as they were intermingled with descriptions of the field of research. Participants floundered in their attempts to place our data, in part because the original study objective was unknown.

Although important across all dimensions of sensemaking, disciplinary and data expertise were key to placing data. Most participants felt that it was easier to describe their data than to summarize and try to understand our data.

My data are much more easier, for sure, because I knew what I was talking about. I didn't have to go through, to understand, which was the quality of the data; I didn't have to understand what it was, the kind of information that this data was giving to me. If I have to go to a database that l've never seen normally and also that is not in my field, it is absolutely much more difficult. (P28) 


\subsubsection{RQ2: How do patterns of data-centric sensemaking afford potential data reuse?}

Some participants believed that only experts from within their same discipline could reuse their data meaningfully, citing the specificity of their data or the need to analyze the data using specific programs. Others stated that appropriate reuse would require a deep understanding of evolving domain research practices; many had difficulty imagining alternative uses for their data outside of their area of research.

I don't think it would be used for a radically different purpose, but I could imagine somebody taking the data and reanalyzing it in relation to a different model of the underlying process, for example. Or confirming the interpretation that we've placed upon the data using our own model...But they would be people who'd be very close to the topic. (P8)

\section{Structures needed for sensemaking and reuse}

A few participants believed that the use of common data structures, terminologies and methodologies within their domains made it possible for their data to "speak for themselves" to others with similar expertise.

I probably wouldn't have to describe it [the data]. Probably they would just get it. (P1)

We observed procedural reasons why data do not speak for themselves, but require additional structures to convey meaning. Participants did not always include column names in their data in order to make them more machine readable, e.g., or they divided datasets into various sub-sheets to ease processing. We also observed that additional information structures, i.e. documents and codebooks, are needed to support reuse for data consumers regardless of domain, as well as to support future (re)use by the original data producer.

Ten years makes a big difference in my memory, too. So, even at the time when I was working in it, I didn't have to refer to the code book, I knew it all by heart. I would have to go back and look at the code book now myself, and that's why it's important to keep the notes on what you're doing with the variables and keep a copy of the survey that was used, the research instrument, those sorts of things. (P11)

Participants described a large variety of documentation and knowledge transfer practices surrounding datasets (Table 7.4). These practices and the formats used to provide additional information are shaped by journal restrictions, metadata schemas and repository 
requirements, and by the perceived usability of the information structures themselves.

Sometimes this additional information is separate from the data; other times it is embedded within the data, i.e. in the case of annotations or descriptions of codes within a spreadsheet. Different data consumers may require different information structures for the same data.

If they're using a different program, I can direct them to a character set, which you can get from this matrix, but the publication of that character set is quite separate but available online. (P23)

So if you start with the README here, then we can take several directions. So, you can delve into the features, what they mean, and you can delve into the feature documentation. You can delve into ways to query it, and do that for yourself, and then you go to all kinds of programming documentation. And then, here I also pointed to tutorials, [..]. And you can read some papers about it and they're also cited...We also have a Slack community with 120 people, and if they have really hard questions, we invite them to Slack, and they are being answered by either me or people who know more about it. (P3)

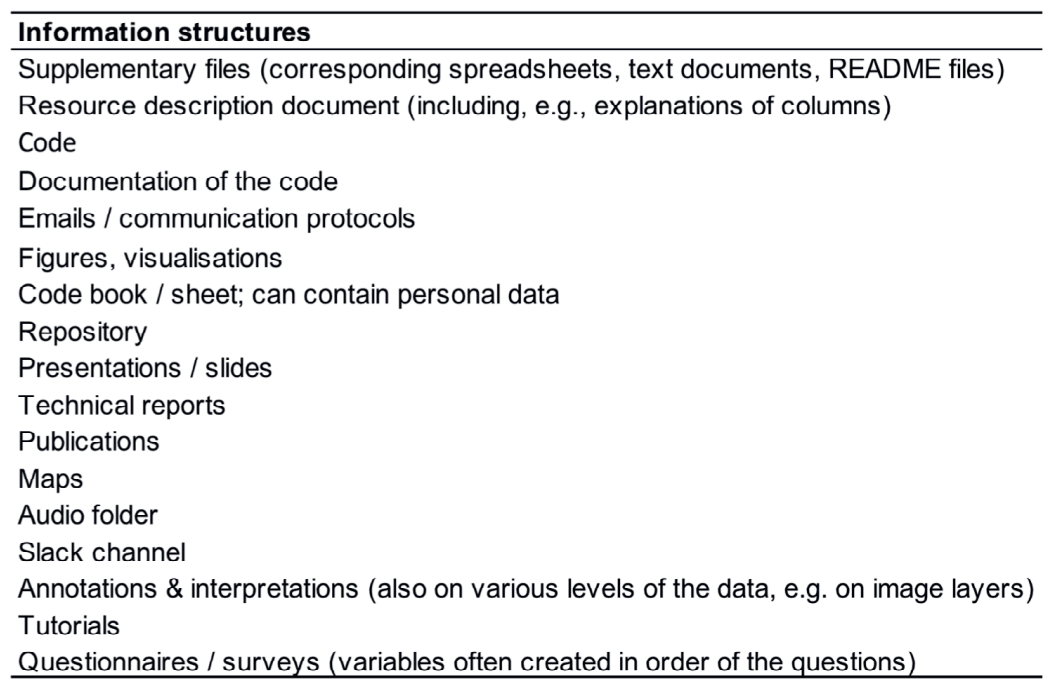

Table 7.4: Information structures supporting sensemaking

The study also revealed attributes which should be present in information structures to avoid losing meaning and to enable data reuse. We present these attributes according to two perspectives which emerged in the interviews: the data consumer's distance to the data and the methodological approach of the original study in Table 7.5 and 7.6. We define "distance 
to the data" in terms of a data consumer's familiarity and expertise with particular data.

Someone "close to the data" will have more knowledge of the data and how they were created; someone more distant from the data will not have this knowledge. In Table 7.6, we focus on two broad approaches to data collection: quantitative and qualitative methodologies. These tables do not aim to present a comprehensive list, but rather reflect the specific work scenarios of our participants.

We asked participants if they would describe their data differently to a colleague with similar expertise, i.e. someone close to the data. Rather than needing less information about the data due to prior knowledge, many participants believe that individuals with similar expertise need more granular information about data creation conditions, prior work which the data builds on, and the potential uses of individual variables. Some participants said that they would not describe their data differently to someone close to the data, emphasizing instead common attributes that would be important, regardless of a data consumer's distance to the data (Table 7.5, Column 3).

\begin{tabular}{|c|c|c|}
\hline Close to the data & Far from the data & No difference by distance to the data \\
\hline $\begin{array}{l}\text { More granular information about conditions, } \\
\text { assumptions, errors, trends, possible questions } \\
\text { the data can answer, variable types, analysis / } \\
\text { programming details, sample creation details, } \\
\text { study objective }\end{array}$ & $\begin{array}{l}\text { More granular information about research } \\
\text { explanation, explanation of all abbreviations / } \\
\text { acronyms, how ratios / errors / columns derived }\end{array}$ & Supplemental materials \\
\hline Benefits / problems of data & Less technical language & Study objective and expected outcome \\
\hline $\begin{array}{l}\text { Previous work that this data builds on, relation to } \\
\text { standards in discipline, out-of-discipline } \\
\text { abbreviation }\end{array}$ & Research explanation & Data collection details \\
\hline \multirow[t]{4}{*}{$\begin{array}{l}\text { Less granular information about field of research, } \\
\text { common abbreviations, data format / structure }\end{array}$} & Tailor to field of interest of the data consumer & Sample details \\
\hline & More general data presentation & Potential use of data \\
\hline & Calculation of ratios/ standard deviation & Usage restrictions, confidentiality concerns \\
\hline & & Explanations of codes, categories, scores \\
\hline
\end{tabular}

Table 7.5: PERSPECTIVE: Information needs related to distance to the data. Someone "close to the data" will have more knowledge of the data and how they were created. Someone "far from the data" will not have this knowledge. Certain attributes did not seem to be affected by distance to the data (Column 3).

So if I'm talking to somebody who is data agnostic or who has not worked in a data science field, my description would be limited to the basic variables, the fields that are of interest to the person...If I'm talking to a data science person or a data scientist who's going to use the data, my description would be more granular. My description 


\section{4 | Chapter 7}

would be more helping the person understand the benefits as well as the problems associated with the data. (P29)

I would maybe shorten up some things and focus on some others. For instance, I would expect that everyone l'm working with expects to code BMI in kilograms and to have birth weight in grams because it's a standard unit for those things in Danish health research...I would tell them more about the study design, because often people I work with are epidemiologists. So there one of the main things would be, where do these 2,000 women come from? Is it data from Denmark or from somewhere else? Is this from last year or from 30 years ago? Things like that, so more complex information so that they can decide if it's relevant for their interests. (P25)

Different methodological approaches also elicited particular details, although these details were not mutually exclusive of each other (Table 7.6). For quantitative data, participants reported needing extensive information about an experimental setup, including how experimental designs differed from the real world environment.

Well I would perhaps mention the size of the pipe diameter. That is something that they're often interested in, because in real pipelines, the pipe diameter is perhaps 12 inch and more, quite large, while in typical labs, you don't have this possibility. (P14) 


\begin{tabular}{|c|c|}
\hline Quantitative & Qualitative \\
\hline $\begin{array}{l}\text { Detailed experimental set-up: } \\
\text { including time period, instrument settings, location, etc.; where } \\
\text { the test conditions differ from real world settings or from } \\
\text { standard procedures }\end{array}$ & $\begin{array}{l}\text { Detailed study set-up: } \\
\text { including time period, description of participant sample, } \\
\text { sample size, mode of interaction (e.g., online or in person) }\end{array}$ \\
\hline $\begin{array}{l}\text { Who did which work (data collection, quality co trol, data } \\
\text { cleaning, code, analysis) }\end{array}$ & $\begin{array}{l}\text { Who did the research; researcher's relationship to } \\
\text { participants and how this was mitigated (e.g., professional role } \\
\text { of researcher / context of recruitment) }\end{array}$ \\
\hline $\begin{array}{l}\text { Are measurements individual measurements or multiple } \\
\text { measurements of the same thing that were aggregated }\end{array}$ & $\begin{array}{l}\text { Questions or schedule for surveys or interviews (including } \\
\text { information about answer modes (e.g., predefined answers or } \\
\text { free text) }\end{array}$ \\
\hline $\begin{array}{l}\text { Which section of the object was measured, on how much } \\
\text { material a measurement was made }\end{array}$ & $\begin{array}{l}\text { Analysis (e.g., type, coding strategy, groupings and narrative } \\
\text { of categorisation) }\end{array}$ \\
\hline Standard error, precision of measurements, uncertainties & $\begin{array}{l}\text { How sample was chosen (inclusion / exclusion criteria), } \\
\text { created, scope and characteristics of sample (e.g., age of } \\
\text { participants) }\end{array}$ \\
\hline $\begin{array}{l}\text { Influencing factors (seasonal differences, external events, } \\
\text { etc.) }\end{array}$ & $\begin{array}{l}\text { How categories were chosen, how scores were created, } \\
\text { variables of focus }\end{array}$ \\
\hline $\begin{array}{l}\text { Standard units of measurement in a field / setting of study } \\
\text { (e.g., instruments - specifications, reliability, how calibrated, } \\
\text { how they work and how they create the data output, software } \\
\text { format used to capture or analyze data) }\end{array}$ & Social context \\
\hline Number of repetitions of experiment & Description of labels / codebook / account of \\
\hline
\end{tabular}

Table 7.6. PERSPECTIVE: Methodological narrative (characteristics are not necessarily unique to either approach)

Key findings from the qualitative perspective include the choice of categories, questions of representativeness and details of the study set-up that influence the data, such as whether participants are required to answer a survey question. Social context also influences how study participants communicate, e.g. in the case of interview participants in conflict areas who may not feel safe enough to respond truthfully to questions.

\subsection{Discussion}

We bring together different perspectives in this study, drawing together our findings about participants' summaries of familiar and unfamiliar data and our observations of how participants engaged with these data. We now synthesize our findings, identifying different patterns of activities and their related data attributes involved in data-centric sensemaking. The sensemaking efforts which we observed can be synthesized into three clusters of activities: inspecting the data, engaging with the data content more deeply and placing data within broader contexts (Figure 7.7). We also examine the relation of the clusters of sensemaking activities to information structures needed for reuse and discuss three emergent themes in the context of this synthesis. Here, we define: 
- Activity patterns as the actions, both physical and cognitive, which people undertake when making sense of data

- Data attributes as characteristics of the data which people interact with as they perform a set of activities

- Clusters as the activity patterns, with their related attributes, which tend to occur together

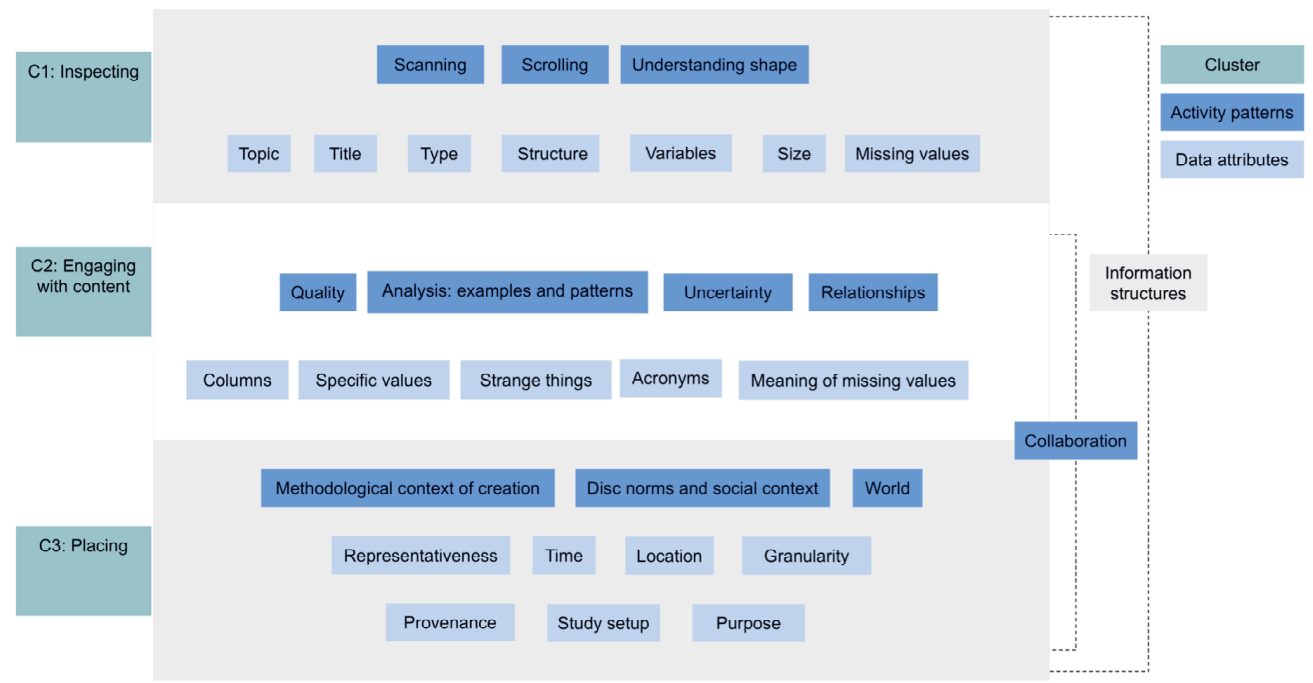

Figure 7.7. Activity patterns and attributes in data-centric sensemaking

C1, inspecting, contains activities and attributes that provide participants with a broad overview of the data, such as understanding the data's general topic, title, structure and format. In the unknown data, we observed that most participants scanned the spreadsheet first vertically to look at the number of rows and to get an idea of missing values and then horizontally to look at the headers.

C2 represents a deeper engagement with the content of the data, including activities such as establishing relationships between columns, performing simple analyses, picking out examples of particular values, conducting quality assessments and trying to understand uncertainties attached to the data, by questioning, e.g. the meaning of missing values or abbreviations and acronyms.

In C3, we observed participants placing data in relation to the world and different contexts. They worked to understand how the data were related to study designs, to disciplinary norms as well as to temporal and geographic considerations to understand the 
representativeness of the content. They questioned, e.g., the level of detail (granularity) presented in the data as well as the data's original purpose.

Our findings show that level one (C1) of Figure 7.7 was mostly done alone; level two and three $(\mathrm{C} 2, \mathrm{C} 3)$ were often solved in collaboration. When participants described their own as well as our data, critical details emerged only after the initial summary description, when both the interviewer and the participant could see and interact with the data together. These conversations moved away from objective descriptions towards describing the complexity of qualitative judgements behind the (quantitative) variables, as well as to rich descriptions of factors influencing the origination of data. This echoes literature in critical data studies, conceptualizing data as the product of sociotechnical arrangements but also as a medium through which conversation and negotiation can occur (Neff et al., 2017).

When discussing their data, participants made use of the information structures identified in Table 7.4 and their related qualities (Table 7.5 and 7.6) across all dimensions as they worked to make their data understood. Many also referenced the lack of contextual information (e.g. purpose, collection methods) in the unknown data as being a stumbling block to understanding.

The importance of needing contextual information to support data reuse, at both the level of the data (Borgman, 2015a; Faniel, Kansa, et al., 2013; Faniel et al., 2012) and of digital collections (Baker \& Yarmey, 2009; Chin \& Lansing, 2004; Lee, 2011), has been extensively noted in the literature. Our work is in line with these findings, particularly in noting the importance of information describing data collection conditions and methodological details. Recent work (Faniel et al., 2019) also draws attention to descriptions of what we term "encodings" in Table 7.3, to describe the codes that participants create and use when working with data.

Koesten et al. (2020) provide a summary of the literature examining particular data attributes for reuse, listing which papers document the importance of certain data and documentation characteristics. While our findings add to this literature, particularly by presenting important attributes along the lines of an individual's knowledge of the data and its creation process (Table 7.5) and the methodological narrative (Table 7.6), our primary aim is not to isolate data attributes needed for reuse, as much work has already investigated this problem. Rather, the work we present here takes the lens of analyzing how those attributes are brought together by the activities they afford, which we group to patterns of sensemaking activities. 
Translating these findings into interaction guidance and subsequently into tools supporting reuse presents a challenge, in part because of the dynamics and context-specific nature of working with data (Kross \& Guo, 2019; Muller et al., 2019). The in-depth descriptions of study set-ups, purposes of data collection and domain specific knowledge brought by our participants underscores this problem. As a way to address this challenge, Figure 7.7 can be viewed in the context of work using design patterns in areas such as software engineering (Gamma, 1995), user interface design (Granlund et al., 2001), or ontology design (Gangemi \& Presutti, 2009).

This approach identifies high-level patterns as a way to provide repeatable solutions to recurring design problems. This creates possibilities for a level of formalization that enables the development of flexible designs and tools. Our results are in line with (Boukhelifa et al., 2017; Koesten, Kacprzak, et al., 2019; Marchionini et al., 2005), who see flexibility as being key to supporting real-world data workflows. Figure 7.7 therefore represents a patternsbased approach to conceptualizing the processes involved in the initial stages of data-centric sensemaking.

To further contextualize Figure 7.7 and to illustrate how our findings could spur design efforts, we discuss three specific themes that emerged in our research at the level of each identified cluster. For each theme, we present design recommendations. The recommendations we propose exist in parallel to research in information visualization (Shneiderman, 1996), which suggests visual support for a high-level taxonomy of data tasks and types. Our study brings a deeper perspective to understanding information needs focusing on structured data, suggesting a wider variety of data-related tasks undertaken by users, which may or may not be supported through visual exploration. Our recommendations build on what we have learned about researchers' sensemaking activities and workflows; our aim is to disrupt these workflows as little as possible. We therefore propose functionalities and approaches to support sensemaking that could be integrated within analysis tools already used by researchers, such as common programming languages or libraries.

\subsubsection{Understanding shape}

When inspecting a dataset for the first time, see Cluster 1, participants either discussed the data in a linear fashion, addressing each attribute individually before moving to the next, or they took a more interwoven approach, mixing descriptions of dataset attributes with analyses and questions. This interwoven approach also has overlaps with activities in the second cluster of Figure 7.7. 
As they engaged in inspecting activities, participants aimed to arrive at an overview, to create a high-level representation of the entire dataset in their head while engaging with it (see also (Koesten et al., 2017). We observed different levels of focus in this process. Participants alternated between "zooming out" to describe the data at the level of the entire spreadsheet, e.g. the number of observations or format of the data, and "zooming in" to look at specific cell values or individual parts of the data. Participants adopting a more interwoven approach tended to engage in the process of zooming in and out more often than those using a linear approach.

This desire to understand the data as a whole has parallels in the information science literature, where the need to understand an entire information collection at a high level has been mentioned (Rieh et al., 2016). Discussing the visual aspects of sensemaking, Russell (2003) also mentions the need to understand what is in a whole collection. White and Roth (2009) recommends allowing users to filter, sort and explore different views of information on demand for complex search tasks. In our study, the information is distributed among the cells of the dataset, the structure and organization of the data, as well as any related information structures.

\section{Recommendations}

Understanding the shape of a dataset can be supported through interface design and functionalities in a number of ways. Our results show that data needs to be understood as a whole, on the level of the entire dataset. This suggests summarization methods, which can be of textual, visual or statistical nature, that provide a zoomed-out view of the data (e.g. Holland et al., 2018). At the same time, participants also engaged with subsets of the data, particularly individual columns; these patterns could be supported through zooming in via column level summaries, including interactive plots and visualizations at the column level (e.g., this idea is partially realized by Kaggle in their dataset previews ${ }^{29}$ ). Future research could look at different ways of expressing a column-based notion of provenance, such as where the data in a column comes from, how it was created or from where it was derived. Given the importance of scanning and zooming in and out (as mentioned in literature such as Shneiderman, 1996), data search engines and displays should optimize this functionality to make these processes as fast as possible; including horizontal scrolling to accommodate spreadsheets with more columns.

\footnotetext{
${ }^{29}$ https://www.kaggle.com
} 
Similarly, certain types of information structures attached to the dataset facilitate particular sensemaking patterns over others. A README file with a summary of the dataset's size and format may provide the information necessary for a zoomed out inspection of the data; an interactive map of the area where a specimen was collected may be more suitable to a zoomed in approach, as well as enabling the activities described in Cluster 2.

\subsection{2. "Strange things" as an entry point, not an obstacle}

Participants repeatedly encountered and dealt with "strange things" in both data sources, i.e. outliers, errors, missing data, and inconsistencies in formatting. As they wrestled with the unexpected in the data, they engaged in the patterns identified in Cluster 2, such as expressing uncertainty, seeking relationships or performing analyses.

Whereas (Zhang \& Soergel, 2020) describe dealing with conflicts as a barrier to sensemaking, our findings suggest that conflict is a useful and accelerating moment in the exploration of data. The concept that real data is usually messy and complex was internalized by our participants. Participants were neither surprised nor alienated by conflicting data; in contrast, errors and uncertainties were expected and participants applied different analytical strategies to overcome them, a finding also in line with recent literature (Boukhelifa et al., 2017; Neff et al., 2017; Koesten et al., 2017). Participants repeatedly emphasized the need to communicate information about sources of error and possible uncertainties to potential data consumers, although there were a variety of communication methods used to do so, some of which are detailed in Table 7.4. Methods for communicating information about strange things in the data were sometimes chosen arbitrarily or convenience-based. Some of this information was embedded within data themselves, leading to potential problems in machine readability. Others were not linked to the data in a sustainable way, making them unsuitable for long-term preservation of meaning.

\section{Recommendations}

Our findings suggest that errors can be seen as an entry point to sensemaking, as flags to investigate further. This provides an interesting direction to explore for sensemaking functionalities in tools. Rather than flattening out data by making it cleaner, tools could instead flag and highlight strange things to make users more aware of their presence. Column summaries, as mentioned in 5.1.1, could include explanations of abbreviations and missing values, metrics or links to other information structures necessary for understanding the column's content. Datasets should include links to basic concepts (used in the data or in the documentation) such as common practices in code documentation or "the web" (i.e. in Wikipedia / Wikidata) to provide context. Documentation about the narrative surrounding 
these strange things should also be more standardized and linked directly to these flags in a sustainable way.

Other sensemaking patterns identified in $\mathrm{C} 2$ can be supported by customized interactive visualizations. Displaying the entire data, as described in 5.1.1, but highlighting relationships between columns or entities could allow users to more easily pick up relationships between columns. Tools could also display trends and patterns extracted from the dataset and allow users to select those attributes of the data that are of interest. Following this idea, data producers could identify anchor variables, those which they consider most important in their dataset; this could further aid sensemaking activities by focusing summarization efforts.

\subsubsection{Perspectives in placing}

Participants place data and their representativeness in a range of broader contexts (the world, disciplinary norms, methodological contexts of creation). While we present these placing activities separately in Cluster 3 , they can in fact be closely related. We saw this particularly in how participants placed data in terms of a study's methods and their own disciplinary expertise.

Details about data creation are often implicit within a domain's epistemic norms (Leonelli, 2016). Even with the best documentation, this complicates cross-disciplinary data reuse. A data consumer from another domain may not have the experience necessary to understand or evaluate the appropriateness of a particular methodological approach. Additionally, our participants' concept of the details needed for reuse encompassed much more than just a step-by-step process of how a study was conducted. Rather, for both quantitative and qualitative data (see Table 7.6), participants needed details about the entire narrative surrounding data creation, i.e. why a certain method was chosen or the unique, local aspects about a study's set-up and their attached constraints. This need for expanded and robust methodological narratives mirrors recent calls for details beyond those provided by standardized metadata and reporting conventions, particularly for the reuse of qualitative data (Poth, 2019).

We also found that the granularity of these narratives is related to a potential data consumer's expertise or distance to the data, with experts needing more detailed information about study descriptions. Table 7.5 also shows common attributes, aside from methodology, that are important in facilitating understanding, independent of a data consumer's expertise with data, i.e. needing information about study objectives, usage restrictions, and explanations of categories and acronyms. 


\section{Recommendations}

Our findings highlight the need for flexible designs to support placing activities across the three identified levels of placing: the world, disciplinary norms and the study set-up. Rather than designing for a specific type of user, tools should be designed to embrace different levels of expertise, allowing a potential data consumer to drill down to the desired level of detail. Semantic technologies (Balog, 2018) also could be used to link to standardized definitions of disciplinary acronyms or terms, mirroring our recommendation in 5.1.1 to link to external knowledge bases. Geographic information could be linked to a map or country registry to allow judgements of representativeness; a similar approach could be taken for certain disciplinary standards and study set-ups, such as standard experiment conditions, expected result ranges or commonly used confidence levels. Data citations, in particular their associated metadata, can contain detailed provenance information needed for sensemaking, offering another emerging possibility for providing the necessary context for data reuse (Groth et al., 2020).

Our findings across all dimensions emphasize the collaborative nature of data-centric sensemaking and the omnipresent role of information structures throughout the sensemaking and reuse process. It has been suggested that the production and consumption of academic writing can be conceptualized as a form of dialogue (e.g. Lillis, 2011); the broader practice of reusing data could itself be seen as a form of collaboration or conversation between data producers and consumers. The data producer must communicate the many (often collaborative) decisions which influence the creation of a dataset (Mahyar \& Tory, 2014; Neff et al., 2017) to potential data reusers.

A combination of focused documentation practices integrating different media types, together with prescribed interaction flows tailored to the sensemaking practices of both data producers and consumers, could facilitate the conversation implicit in reusing data. These could include solutions with adaptable data representations suited to varying levels of expertise and needs.

\subsection{Study Limitations}

Although they were working in a wide range of disciplinary domains and research related roles, our sample population consisted of a particular type of professional: researchers who have published an article indexed in the Scopus database.

Scopus comes with a skew towards certain research fields; the Arts \& Humanities, for instance, are not as well represented. Scopus has an extensive review process for the 
journals which it selects for inclusion (Elsevier, 2020); and there are roughly an equivalent number of journals from the broadly defined fields of social sciences, health sciences and physical sciences (Elsevier, 2020). While the limitations of Scopus could lead to a potential bias in our sample, the selection criteria we applied also ensured that the sample population met our study requirement of speaking with different types of researchers with data experience.

As the study was conducted with researchers, our findings may not be directly applicable to other individuals. Focusing on researchers met the goals of our study, particularly our aim of examining sensemaking in light of reuse. However, we believe the general sensemaking patterns emerging from this study are to some extent transferable between different skill sets; simply the execution of how these goals are achieved might look different for people with a higher or lower level of data literacy. Nonetheless, the study would need to be repeated with different populations in order to apply our findings more broadly.

Our participants work in a variety of countries; English was not the native language for all. To account for this, we selected our sample from those responding to our recruitment messages carefully to ensure that participants had a high degree of English fluency. While we see the global spread and disciplinary diversity of our sample as a strength of the study, we also recognize that data, research, and sensemaking practices are influenced by social, legal, and economic contexts unique to both country and disciplinary domains.

The sensemaking patterns which we identify could also be limited by the data themselves. Different data may have surfaced different data attributes. By including participants' data, as well as the unknown dataset in the study, we attempted to balance this potential bias. Another potential limitation is that describing their data first might have primed participants for performing the verbal summarization task with the unknown data, influencing the way that they performed the second task. Given that any data description will be based on a participants' prior experience, we believe this is a natural side effect of these types of studies.

Finally, it is important to note that we intentionally did not ask participants to bring metadata for their known data as we wanted to see what types of data, metadata and other contextual information they felt that they needed to bring without being prompted. Similarly, the study design allowed us to capture what participants felt was missing from the unknown data and to identify what additional information was needed. This is especially relevant as data does not always come with complete or accurate or meaningful metadata or enough information for reuse, as detailed in the background section. 


\section{4 | Chapter 7}

\subsection{Conclusions}

In this study, we investigated common patterns in sensemaking activities in initial encounters with data, particularly in light of potential data reuse.

We identified three clusters of activities involved in initial data-centric sensemaking (inspecting, engaging with content, and placing in context), and detailed the observed activities and data attributes relevant in these clusters. This approach provides an avenue to bring focus to design efforts, narrowing down the many technologically feasible solutions to those specifically supporting the sensemaking needs of data consumers. To summarize, the contributions of the paper are:

- activity patterns for data-centric sensemaking;

- a framework of these patterns and their associated data attributes; • user needs for data reuse;

- design recommendations to support the identified activity patterns.

Our work illustrates the large space for future research trajectories in this area to validate and apply insights within different contexts of data-centric work practices. This could include investigating the identified activity patterns with different data or with individuals working outside of research. Other work could focus on how to apply the detailed insights and recommendations to existing user workflows. Such work could focus on determining the best way to present and allow interaction with data to facilitate sensemaking. Similarly, such work could explore the integration of our findings into existing services and platforms, particularly with regard to multidisciplinary data.

Sensemaking allows individuals to create rational accounts of the world which enable action (Maitlis, 2005). In this work, data-centric sensemaking enables a particular type of action: the reuse of data in research. Understanding how people make sense of data, and exploring designs to support these practices, therefore, plays a key role in realizing the potential of data reuse.

\section{Acknowledgements}

This research is partially supported by the Data Stories project, funded by EPSRC research grant No. EP/P025676/1 and by the NWO Grant 652.001.002 Re-search: Contextual search for scientific research data. 
Talking datasets: Understanding data sensemaking behaviors | 245 


\section{CHAPTER 8}




\section{Conclusions}


248 | Chapter 8 


\subsection{Introduction}

This dissertation adopted a theoretical approach integrating user-centered models of information seeking and core STS concepts to present evidence of practices of data discovery, to examine how researchers across disciplines discover, make sense of and use data which they do not create themselves. It proposed recommendations for systems development and design, which are further synthesized in Chapter 9, and described in detail an openly-available dataset which can be used as the basis for future research. This chapter summarizes the work presented in the preceding chapters and discusses recurring themes according to the theoretical framework. It closes by considering potential limitations of the research as a whole and proposing directions for future research.

\subsection{Review of chapters}

Chapter 2 presented the foundations and details of the theoretical framework used in Chapters 3-5. The chapter opened by addressing how information seeking has been studied in both information science and science and technology studies. It then reviewed influential user-centered models and discussed how STS has approached key elements implicated in these models, namely users, communities and context. The chapter brought together two cornerstones drawn from these perspectives to structure the framework - a modified version of the information journey model (Blandford \& Attfield, 2010) and a series of questions developed to investigate common themes drawn from the reviewed STS literature.

Chapter 3 applied an early iteration of this framework to review the documented data discovery practices of users of observational data across five broad disciplinary groups: astronomy, earth \& environmental sciences, biomedicine, field archeology and the social sciences. The review was undertaken to find commonalities, but it also documented a diversity of data needs and practices, highlighted the absence of particular communities in the literature and concluded that a theoretical framework based on information retrieval alone is insufficient for deeply understanding practices of data discovery.

Chapter 4 brought together bibliometrics and semi-structured interviews to further explore data discovery practices. A bibliometric analysis of the literature corpus collected in the third chapter revealed the technical bias and distributed nature of the discourse surrounding data search. Interviews with data seekers connected two different perspectives - that of data seekers and systems designers, whose input informed the development of the interview protocol. The chapter called for a broader understanding of both the individuals seeking and 
using data, as well as the data needed in research. It also recognized the liminality of practice, finding that data discovery spans the thresholds of other practices, occurring, for example during the course of data sharing and data management.

The study presented in Chapter 5 employed a broader approach - a globally distributed multidisciplinary survey, with nearly 1700 respondents - to explore data discovery practices at a larger scale. The survey questionnaire was designed to probe the role of social interactions in seeking data and to explore relations to other practices, such as searching for academic literature. An initial quantitative analysis and a more extensive exploration of the qualitative data was used to consider commonalities in practices, as well as to examine differences, looking at how data needs and search practices vary not only by disciplinary domain but also by types of data uses. It proposed a typology for data (re)use and considered how communities of data seekers can be conceptualized in new ways.

Chapter 6, published as a data descriptor paper, provided detailed contextual information about the data collected in the survey. The chapter was followed by a reflective piece in which I considered the work needed to prepare and share data, the affordances and duplication problems data papers present; and the drawbacks and benefits of implementing standard formats.

The final empirical chapter focused on a particular part of the data discovery process: datacentric sensemaking. This collaborative study combined in-depth interviews, a think-aloud task and a screen recording analysis with researchers, as they summarized and interacted with both familiar and unfamiliar data. We identified and detailed common patterns of datacentric sensemaking across three clusters of activities: inspecting data, engaging with content, and placing data within broader contexts. The chapter also explored the role of contextual information structures and collaborative practices and proposed specific design recommendations to facilitate sensemaking and subsequent data reuse.

\subsection{Recurring themes}

Principle findings and themes recurring across this body of work are now synthesized and presented according to the theoretical framework detailed in Chapter 2, which is visualized again in Figure 8.1. The framework combines elements of a modified version of the information journey model - users and their needs, discovering, evaluating/sensemaking and reusing - with guiding questions developed to tease out concepts drawn from the STS literature regarding users, communities and context. 


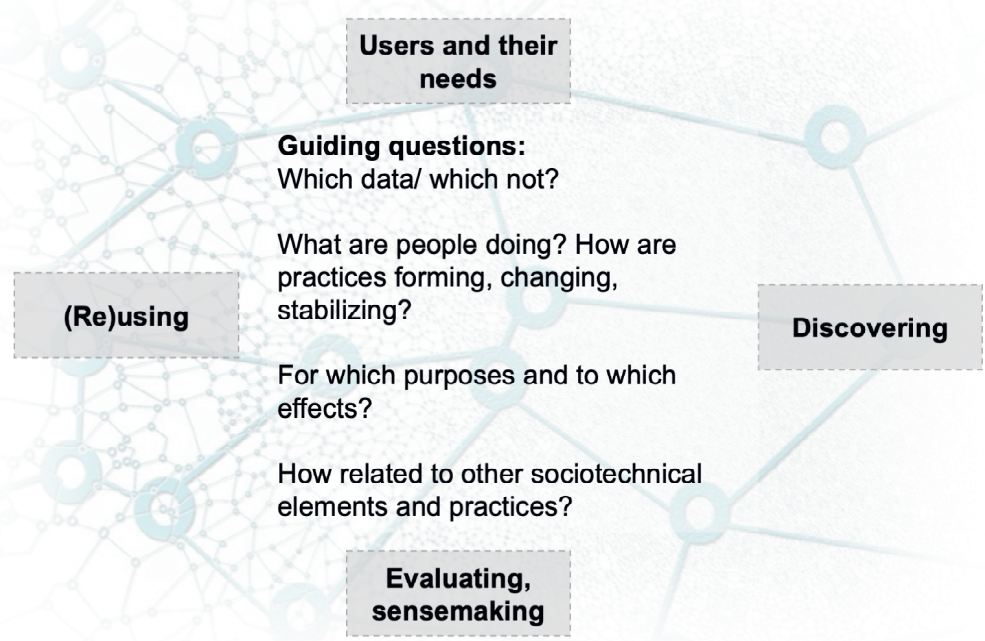

Figure 8.1. Visualization of the theoretical framework for studying data discovery practices presented in Chapter 2.

These STS-inspired themes, highlighted again in Box 8.1, emphasize that examining objects of research from different perspectives helps to uncover actual practices of use, to reveal the diversity and multiplicity of practice and to explore relationships between dynamic, sociotechnical elements.

(1) The importance of examining objects of research from different perspectives.

(2) Diversity and multiplicity are woven throughout scientific and technological practice.

(3) Users, communities and context are reciprocally shaped by dynamic elements in relation with each other.

Box 8.1. Unifying themes drawn from STS literature reviewed in Chapter 2.

Mirroring the organization of the majority of the empirical chapters of this thesis, the following synthesis is structured according to the elements of the modified information journey model. The themes and questions drawn from STS perspectives are present in the analysis and discussion below and are further explicitly discussed in Section 8.3.5, using specific examples drawn from the work. 


\subsubsection{Users and data needs}

\section{Users are not homogenous}

Researchers are not the only individuals seeking and using secondary data; multiple individuals - librarians, literature managers, students, people working in industry - also engage in data discovery (Chapter 4). Nor are data seekers always the end users of data, as evidenced in both Chapter 4 and in the results of the survey, where respondents report relying on both graduate students and support professionals to find data.

Researchers act in multiple roles: as data seekers and data reusers, but also as data collectors, data describers and data sharers. This multiplicity influences how they and others discover and use data. When describing data, researchers do not always follow standardized metadata conventions, even within communities with specific description schemas (Chapters 4,7 ); these practices negatively impact both discovery and evaluation efforts. When documenting their own data, researchers include encodings which may support their own analysis, but which are not always helpful, or even sensical, for future data reusers (Chapter 7). Research support professionals, who also conduct their own research, appear to use a greater variety of data sources more frequently than do individuals who identify as researchers (Chapter 5).

Disciplinary domains are diverse, although this diversity is often overlooked, as researchers are grouped together in broad disciplinary categories (Chapter 3 ). Classifying a researcher's disciplinary domain is complicated, even for researchers themselves; more than $50 \%$ of survey respondents identify as belonging to two or more disciplinary domains. This could indicate that a single domain does not adequately capture the complexity of respondents' expertise or it could reflect interdisciplinarity in research.

\section{Communities}

The research presented across this dissertation encourages a multi-dimensional way of thinking about research and data communities. Data practices are situated within and shaped by disciplinary norms, but communities are not defined by discipline alone (see Chapter 2). Other recent work suggests that data communities can form around shared data (Cooper \& Springer, 2019), or, as suggested in Chapter 3, potentially around broad types of data. Researchers need multiple types of data for their work; it follows that they also simultaneously belong to multiple data communities. 
Communities are dynamic, and can have different norms regarding data sharing, data ownership, data collection and data description; individuals juggle these different norms as they act within different community contexts (Chapter 4). Communities may also form around common uses for data or around common research methodologies, such as those incorporating data science. Chapter 5 suggests that these communities of use may have similar data needs, where specific contextual information supports particular data uses and research stages.

\section{Non-users}

The focus of this dissertation is on the practices of people who discover and use data in research; some conclusions, and some questions, about non-use also arise. The practices of researchers in certain disciplinary domains are over-represented in the literature, while other disciplines are discussed at varying levels of detail or not at all (Chapter 3). This could indicate that individuals in other disciplines are not seeking and reusing data, perhaps because data reuse does not match community or research norms. It could also mean that the practices of these other disciplines are not as well documented or that they are so seamlessly integrated with other practices that they have become invisible.

The challenges researchers face when seeking and using data can shed some light on the non-use of both systems and data. Not all data are accessible or findable; nor are all data available digitally (Chapter 4 ). These challenges are one of the reasons research participants engage in strategies which do not involve interacting with a system. However, even within domains which have well-established infrastructures to support data discovery, researchers do not use them exclusively (Chapters 3,5$)$. This could be because other sources and strategies offer affordances not supported by these systems, such as collaboratively understanding data (Chapters 5,7 ) or growing social networks and forming collaborations (Chapter 4).

Work in STS proposes that use and non-use exist on a sliding scale (Wyatt, 2003). Researchers use multiple data sources at varying frequencies and need multiple types of data (Chapter 5). This suggests that although an individual may at times be a user (of a system or of data), he or she is also, at times, a non-user.

\section{Specificity, multiplicity and diversity of data needs}

All chapters highlight both the specificity and diversity of data used in research. Chapter 3 , for example, details the specific requirements of observational data users, who need data from particular locations (geographic, anatomical or astronomical), at particular resolutions 
or collected with particular instruments. Specific information about data, provided either through metadata or other information structures, helps to support sensemaking (Chapter 7) and particular data uses (Chapter 5).

Researchers need multiple data for multiple purposes; approximately half of survey respondents selected needing more than one type of data. Some bring together various types of data centering on a particular subject; others need data with similar structures and formats, regardless of the topic (Chapter 5 ).

The diversity of needed data is also apparent in the interviews described in Chapter 7 and Chapter 4 . Some researchers brought entire packages of codebooks, spreadsheets and documents to the interview, while others brought a single spreadsheet (Chapter 7 ) or considered only the metadata describing other datasets to be data (Chapter 4). This diversity underscores the relationality of data, emphasizing that what serves as data for one individual within a particular epistemic community will not count as data for another (Leonelli, 2015; Borgman, 2015a).

\subsubsection{Discovering}

\section{Success}

Data discovery is challenging. Success depends on a variety of sociotechnical factors, including being able to access data so that they can be downloaded and analyzed (Chapters $3,4,5)$. Determining who can access data, under which rights and restrictions, is a complex process, involving legal considerations, as well as considerations about researchers' future projects and opportunities for professional advancement (Levin \& Leonelli, 2017).

Data discovery is also complicated by the fact that data are distributed across sources, including general and domain-specific data repositories, government portals, project websites and museum archives. Data seekers must combine strategies and become fluent with a variety of online interfaces, the more general of which may not support the specificity of data needs (Chapters 4, 5). Although some researchers are aware that incomplete and unstandardized metadata complicate data discovery (Chapter 4), the majority focus on the challenges such metadata pose for adequately understanding and evaluating data.

Despite these challenges, and a recognition that the process is difficult, interview participants in Chapter 4 believe that their attempts to find data are, on the whole, eventually successful. If they are not, participants believe that the data don't exist, they make do with 
what data they can find, they give up searching, or they create their own data. This is in line with other findings (Faniel et al., 2016) which report satisficing behaviors, where researchers settle for the data they can find and access, rather than ideal data.

\section{Diversity of sources and strategies}

Mirroring the distributed data landscape, researchers make use of a variety of sources to discover data. Some sources are specific to particular disciplinary or research communities, i.e. journals or domain repositories, and some have wider audiences, i.e. general search engines. Even within disciplines which have well-established resources, researchers use an array of data sources to discover and access data (Chapters $3,4,5$ ).

Three sources emerged to be particularly important in Chapter 5, across disciplines: the academic literature, general search engines, and disciplinary data repositories. Although selected most frequently (Chapter 5 ), researchers use the academic literature in perhaps unanticipated ways, such as plucking data from figures, tables and graphs (Chapters 3, 4, 5). Although they encounter data serendipitously when reading the literature, they also turn to the literature with the goal of discovering data (Chapter 5).

General search engines, such as Google, are also used in numerous ways: to conduct broad searches, but also to locate known data repositories and to find well-known and commonlyused data (Chapters 4,5). Part of the allure of general search engines could be their familiarity and ease of use or the ubiquity and freely-available nature of such tools. Even though general search engines are frequently used, it does not mean that they always retrieve the desired results. Both Chapters 4 and 5 report mixed success with Google, perhaps because of a lack of specificity in search options or quality in content (Chapter 4).

Disciplinary data repositories are another key source, especially within domains which have well-developed infrastructures, such as astronomy and the environmental sciences. Chapter 5 finds that researchers may be more likely to view data and literature discovery as a single, seamless experience, when domain infrastructures link literature and data search.

\section{Interwoven with other practices}

Other chapters also surface the interwoven nature of data discovery with research and search practices. Researchers discover data serendipitously within the course of managing and sharing their own data (Chapters 4,5 ) and through other common research practices, such as attending conferences. Data search strategies are also interwoven with and reflective of common literature searching strategies, such as citation chaining (Chapter 5). 
Data citation is a fairly immature practice in many disciplines (Robinson-Garcia et al., 2016), and standards and best practices for citing data are still being developed. In addition to technical challenges, such as citing data at varying levels of granularity (Pröll \& Rauber, 2013), little is known about why people actually cite, or do not cite, data (Silvello, 2018). Despite these unknowns, nearly eighty percent of survey respondents who use literature as a data source report following citation trails from the literature to data. This strategy has many possible failure points, ranging from authors not citing data which they use to unstable infrastructures for data storage or links between data and publications.

\section{Social interactions}

Across studies, the importance of consulting with personal networks and engaging in various social interactions to discover and access data is clear. Researchers find data through serendipitous and strategic interactions with colleagues, collaborators, supervisors, data authors and support staff (Chapter 4); only $15 \%$ of researchers in the survey report never making use of personal networks to discover data. Relying solely on personal networks may pose its own risk - creating social "filter bubbles" which potentially limit the discovery of novel data (Chapter 4).

Certain interactions appear to have different affordances and be more relevant for particular domains. Chapter 5 suggests that contacting data creators is an especially important strategy in accessing data, for example, while other activities, such as forming new collaborations or attending conferences, are more discipline-dependent in both accessing and discovering data.

\subsubsection{Evaluating / Sensemaking}

\section{Contextual information}

Researchers bring together contextual information from various sources, both external and internal to the data, to evaluate and understand data for reuse. This information describes not only how data are created, but it also explains how data are documented and represented, by detailing, i.e., the meaning of missing values or of specific abbreviations. Details about methodologies, data collection conditions, data provenance, access restrictions and the reputation of data sources are important considerations for researchers across all studies. 
The necessary contextual information may vary according to data uses or levels of expertise. Chapter 5 reports initial signals that content-related information, such as the original purpose of the data or topical relevance, is important in the project preparation stage of research. Chapter 7 suggests that experienced researchers may need more detailed contextual information than individuals with less disciplinary or data expertise. Particular ways of packaging this information may also support particular sensemaking activities. README files may be enough to support high-level inspections of data, whereas more granular representations of trends or patterns support more detailed understandings.

\section{Quality and trust}

Researchers combine contextual information, examinations of data characteristics and their own engagements with data to establish data quality and trustworthiness. A lack of errors and the overall completeness of data, as well as the reputation of both the data creator and source, are important factors in determining quality (Chapters 4,5 ). Detailed explanations of possible sources of error and potential biases are key to understanding anomalies or "strange things" about data (Chapter 7).

Work across chapters finds that researchers are not necessarily surprised or alienated when they encounter the unexpected in secondary data. Researchers tend to know that real data are messy and complex. They realize that while anomalies can be indicative of possible mistakes or methodological weak points, they can also stem from different causes, such as modifications to long-running experimental designs (Chapter 7). To understand and detect anomalies or uncertainties, researchers apply different strategies, such as performing statistical checks or calculating ratios between variables (Chapter 4).

Errors also play a role in trust development. Small errors make some wary of data (Chapter 4), while for others, a complete lack of errors could indicate that the data have been tampered with or modified (Chapter 5). As when establishing quality, researchers like to have access to the data, to be able to examine and tinker with them, in order to develop trust. Coming to trust data is a social endeavor (Yoon, 2017b), as is mirrored here in the importance of the reputation and transparency of the data source (Chapters $3,4,5)$ or in the perceived trustworthiness of gold-standard datasets which have been accepted and endorsed by particular communities (Chapter 4).

\section{Social interactions}

Researchers consult their personal networks, contact data creators, attend conferences, interact on discussion forums and forge new collaborations as they work to make sense of 
data. Some of these strategies appear to be more important than others, influenced by disciplinary norms regarding collaboration and by communication infrastructures (Chapter 5); the importance of interacting with personal networks remains strong across studies and disciplines, however.

Chapter 7 describes the role of social interactions through a different lens, highlighting the conversations and questioning that occur - in this case between research participants and interviewers - during sensemaking activities. These types of collaborative efforts appear to be more important when researchers work to develop deeper understandings of data, rather than during initial data inspections.

\section{Clusters of sensemaking activities}

Making sense of data involves combinations of people, actions, contextual information and data attributes. All chapters show that researchers consult sources such as the literature, data documentation and their own personal networks to understand data for reuse; they also engage in exploratory data analysis or triangulate data from multiple sources. Chapter 7 identifies clusters of researchers' common activities when working to understand data inspecting, engaging with content more deeply, and placing data in context. It then situates these activities in relation to common data attributes, as well as to contextual information and collaborative actions.

When inspecting data, researchers rely on data characteristics, information and activities that provide a broad overview. They need information about the data's general topic, structure and format; they engage in actions such as clicking on cell values, sorting and reformatting data. When engaging more deeply with data, researchers establish relationships between variables, provide examples and perform numerical checks, as they question the meaning of anomalies or unknown abbreviations. As they place data in relation to broader contexts, researchers try to understand how data are related to study designs and disciplinary norms; they also make use of temporal and geographic information to establish the data's representativeness.

\subsubsection{Reusing}

\section{Complex and changing data uses}

Chapters 3 and 4 distinguish between background uses of data, undertaken to support research, and foreground uses, which drive new research (Wynholds et al., 2012; Wallis et al., 2013). Classifying data uses according to this typology presents challenges. $A$ 
background data use for one community, such as benchmarking, could be construed as a foreground use in another. Although data uses may be common, their enactment within particular communities will not be uniform or have the same meaning (Chapter 5).

One example of this complexity can be seen in data integration. Chapter 3 emphasizes that researchers who use observational data integrate data across sources and contexts; the challenges involved in data integration are often mirrored within discussions about data interoperability (i.e. Wilkinson et al., 2016). Data integration did not arise as a common use in the interviews in Chapter 4, however; nor was it a frequently selected use in the survey study.

This could be explained in different ways. Perhaps the survey question was not worded clearly enough, or perhaps researchers do not call their work of bringing together diverse data "integration." Another possibility is that integrating data is a use that is only relevant for particular data communities or types of data, or that it does not occur as frequently as other data uses.

The research in this dissertation adds to the list of previously well-documented uses of data in research, identifying practices such as using data to obtain an overview of a topic, to experiment with new data science or visualization methods, to prepare grant proposals or in teaching. Chapter 3 finds that background uses for data are more commonly documented than foreground uses, and that other data uses, such as using data in teaching, are not well studied.

Yet the majority of survey respondents $(71 \%)$ report using data as the basis for new research, and more than half report using data in teaching. While these findings are reflective of the behaviors of the survey respondents, this could also indicate a broader change in data practices. Chapter 4 suggests that this could be the case, as interview participants report needing data due to engaging in new projects, finding new jobs, teaching new classes or wanting to experiment with data visualization, given the increasing amount of openly available data. Changing uses for data could also feasibly be the result of changes in funding calls, which increasingly emphasize using data science methodologies (i.e. National Science Foundation, 2020), or policies encouraging data sharing and reuse.

\section{Changing classifications for data use}

Classifications of data use are also changing. Rather than conceptualizing data uses as background or foreground, Pasquetto and colleagues propose a typology that places types 
of data use on a continuum between comparative uses, i.e. ground truthing or instrument calibration, and integrative uses, i.e. bringing together data for new analysis or to identify new patterns (Pasquetto et al., 2019). They find that integrative uses occur very rarely, perhaps once in a research career, whereas comparative uses occur fairly frequently. Integrative uses, which they classify as emergent practices, require more expertise, often necessitating collaborations with data creators, as opposed to more routine comparative data uses.

This classification could provide another explanation for the fact that survey respondents in Chapter 5 selected data integration at a lower percentage than other uses. Data integration may still be an emergent practice in some communities; it could also require specific expertise and collaborations that are not easy to acquire for all researchers. Pasquetto et al.'s work also supports the idea discussed here of understanding use and non-use on a continuum of engagement, rather than as binary, either-or actions.

Drawing on the findings and analysis from the survey, Chapter 5 proposes another typology for data uses, emphasizing that researchers make use of data in multiple ways, throughout phases of research and academic work. This typology also stresses the interwoven nature of practice, finding that data analysis tasks are likely to occur throughout all phases of research. The typology shows that certain types of data are associated in the survey results with certain data uses. This resonates with the idea that data are defined as such by their use at particular points of time in the inquiry process (Leonelli, 2015).

The viewpoint represented in this typology is very task-oriented. Other work also demonstrates that data discovery is shaped by particular tasks (Koesten et al., 2017), and work across the dissertation shows that the uses presented in Chapter 5 are prevalent. Such typologies do not account for other roles that data play, however, such as those identified in Chapter 4, where data were found to act as hubs for collaboration or creativity, or the role that data play in binding together various communities.

\subsubsection{Revisiting STS perspectives}

The identified themes drawn from the STS literature which informed the development of the theoretical framework (Box 8.1), are apparent in the above synthesis. Here, I draw out specific examples of how these themes are present in this work. 
First, the approach taken here arguably provides a different perspective for examining practices of data discovery. It is not based on a particular user-centered model; nor is it entirely rooted in a single theory from STS. The collaborative work presented in Chapter 7 also provides another viewpoint for understanding data-centric sensemaking, both methodologically as well as theoretically.

By taking the perspective of the data seeker, this work also emphasizes views of objects, technologies and practice that may not be given as much import from viewpoints more external to the practice. Differing perspectives regarding the role of metadata provide an example. Implementing standardized metadata is often central to discussions regarding data discovery amongst systems developers, as a way to enhance data findability (i.e. Noy et al., 2019). Researchers are also cognizant about the need for standardized and rich metadata creation, although they emphasize the impact of non-uniform or incomplete metadata on sensemaking, rather than its influence on the correct retrieval of data in a search setting.

The second and third themes shown in Box 8.1 are also apparent throughout the dissertation. All studies find that communities, data, contextual information, data uses, and discovery practices are both diverse and multiple. They are dynamic, and co-shape each other, as well as the data infrastructures, policies and other (re)search and data practices which surround them.

The role of citations in locating relevant data provides a good example of the reciprocal nature of discovery practices. Researchers apply, or attempt to apply, a tried-and-true strategy for literature searching to finding data: citation chaining. They do this, despite the fact that data citation is not a common practice in many disciplinary fields. At the same time, repository and infrastructure managers hasten to develop standards and technologies to facilitate data citation. These actions could further support and normalize the practice of citing data, although the exact meanings of data citations, particularly regarding aspects such as credit and attribution, are unclear and emergent (see Borgman, 2016).

\subsection{Limitations}

Most of the chapters include individual sections or comments regarding the limitations of particular studies. Here, I discuss limitations which arise when considering the work in its entirety, many of which center on issues of scope. 
The collaboration with Elsevier facilitated recruiting participants across studies; it could also have led to potential bias. In Chapter 4, participants were selected from a pool of individuals who had visited the DataSearch website and indicated an interest in providing feedback. It is possible that interview participants may have taken part in the study in order to engage more with Elsevier regarding the design of DataSearch. As this search engine was still in development, it is also possible that it was only well known in certain disciplines or communities. However, perhaps because DataSearch is a freely available tool which indexes a variety of data, participants ranged in their professional roles, disciplinary domains and geographic locations.

Research participants for the studies described in Chapters 5 and 7 were recruited from a list of authors who have published an article within the last 3 years that is indexed in Elsevier's Scopus literature database. While Scopus has a disciplinary skew towards certain disciplinary domains (Mongeon \& Paul-Hus, 2016), it also has an extensive review process for the journals which it indexes. Ten percent of the 22,800 source titles indexed in Scopus are published by Elsevier; the remainder are produced by other publishers across the world (Elsevier, 2020).

Research participants themselves are likely to represent an actively-engaged portion of the general population. Participants were confident in their English-speaking abilities and were able to take part in research conducted in-person, via video-conferencing technologies, or in an online survey environment. Limited information is available about people who did not respond to requests for participation, complicating analyses of under-represented populations. The findings presented across studies are descriptive, not predictive, and represent the practices of participants themselves.

Individual studies built on and informed each other. In general, this is a strength of the research; a drawback is that any bias present in an earlier study would also be present in subsequent work. The literature corpus collected in Chapter 3 , for example, was used in the bibliometric analysis in Chapter 4. Any bias in the collected literature, due to the content coverage of literature databases or the keywords and search strategies used, would be present in both of these studies. Similarly, interview participants for the sensemaking study in Chapter 7 were recruited from the broader pool of survey respondents. While this recruitment strategy enabled us to reach our targeted population, it could also have limited the diversity of voices present in the research in Chapter 7. 
The above points suggest the possibility of a limited scope of the work. Focusing on practices of researchers, rather than simultaneously investigating practices of other actors, i.e. systems developers, could also be another limitation. Conversely, the analytical approach taken here could have been too broad. Although I highlight differences, as well as commonalities, throughout the chapters, it is possible that some of the complexities involved in participants' practices were missed or not analyzed in depth with this approach.

\subsection{Future research and open questions}

While the work presented in this thesis addresses the research questions posed in Chapter 1 , it also raises open questions and possibilities for future work, which could be addressed using a range of methodologies.

First, there is room to further explore the diversity and differences between the data discovery practices of particular groups. Given the emergence and evolution of data-related roles in research, i.e. data stewards, data managers and archivists, the practices and roles of "support" professionals should be further investigated. The survey data, described in Chapter 6, offers some insight into these differences; this could be used as a starting point to inform such future research. The practices of early career researchers or of researchers in less quantitative disciplines, i.e. arts \& humanities, could also add to the discourse surrounding data discovery and reuse, as could teasing out within-group differences, such as differences within a broad disciplinary group, i.e. the social sciences.

Future work could also explore and test the idea of different types of data communities proposed here. How do communities form around shared data, common research methodologies or data uses? Where are the differences and overlaps in belonging to data communities which are more loosely defined, i.e. users of observational data (Chapter 3 ), and more intense, structured data communities? Case studies and interviews, particularly focusing on the development of shared feelings of identity, could help to answer these questions.

The list of data uses proposed in Chapter 5 also needs to be tested and potentially broadened to see what other types of data uses are relevant in research. Conceptual work could also be undertaken to further explore how these uses align with other recently proposed typologies for data reuse (i.e. Pasquetto et al., 2019). 
Although this dissertation has contextualized both use and non-use, bringing focus to instances of non-use, of either secondary data or of discovery systems, offers another possible avenue for study. Further understanding the reasons behind non-use within various communities could help to reflect the complexities of using and searching for data.

Qualitative methods could help to explore non-use in depth, although identifying populations of non-users may be challenging.

The openly-available survey data also has the potential to answer future questions.

Differences in the survey data could be explored along demographic variables, such as geographical location or career stage. Multi-level models could be used to further explore the influence of data uses, data types, or disciplinary domains or to test for correlations between, i.e. the frequency of using a particular data source and the type of data needed or various data uses. An open question is to explore how representative the survey data are of the behaviors of broader populations. The data could also serve as a baseline for comparison in the future, as the data discovery landscape continues to evolve.

There is also room for deeper understandings about how data seekers interact with particular technologies and systems. As in Chapter 7, screen recordings, observations, (and potentially query log analyses) could be used in conjunction with interviews or case studies to learn more about how individuals search for data and why they do so.

Finally, different theoretical approaches could also complement the approach taken in this dissertation. Examining data discovery through an infrastructural approach (as in Edwards, 2010) could help to further uncover the hidden work of various actors in both discovery and reuse. Alternatively, taking metadata as a starting point for exploring the sociotechnical networks involved in data discovery could provide yet another perspective to that of the researcher.

\subsection{Closing thoughts: Findable and reusable?}

This dissertation has presented evidence of practices of data discovery across disciplines, exploring how researchers discover, make sense of and reuse data in their work. It has detailed a dataset that can be used to further explore these practices and has provided recommendations for systems development, as further discussed in the following chapter. As a whole, this research has contributed to the broader discourse on data discovery, sharing and reuse and the position of these practices within scholarly communication and knowledge production. 
This research has shown that discovering and reusing data involve webs of interwoven practices, actors and technologies. Researchers make use of social connections - with data authors, peer networks and broader communities - and interact with various (digital) technologies to locate and understand data. Their discovery practices are shaped by data characteristics, as well as the hidden work involved in creating data and making them portable, i.e. processes of data description, sharing and standardization. Their practices both influence and are influenced by discovery systems and data infrastructures, including interface designs and how data are linked to other objects to provide context.

Finding and reusing data are therefore not reducible to performing keyword searches online or pressing a button to download data. Rather, data discovery and reuse are deeply sociotechnical practices which rely on dynamic relationships between people, technologies, materials and policy. 


\section{CHAPTER 9}


Recommendations and

Valorization 
268 | Chapter 9 


\subsection{Introduction}

This final chapter discusses the practical applications and reach of the work presented in the dissertation. First, recommendations made throughout the thesis, primarily for systems development and design, are brought together and synthesized. I then consider potential applications for policy development and end with a valorization statement, as required by the Maastricht University $\mathrm{PhD}$ guidelines, where I reflect on the reach and uptake of this research.

\subsection{Recommendations}

\subsubsection{Design recommendations}

\section{Recommendations to support data discovery and reuse}

Box 9.1 synthesizes the recommendations made for systems designers and repository managers proposed in Chapters 4 and 5 . The recommendations can be classified along three lines: suggestions centering on data supplies and metadata; those which require community engagement and facilitate social interactions; and those targeted toward the design of user interfaces. The boundaries between these categories are porous. Italicized recommendations in Box 9.1 in particular rely on community engagement and are designed to encourage communication, although they are categorized as metadata recommendations.

The first section in Box 9.1, data supplies and metadata, builds on the recommendation made in Chapter 4 to support the broad landscape of researchers' data needs. To do this, designers of discovery systems could index data created both within and external to academia, as well as data from both disciplinary and multidisciplinary sources. This recommendation is echoed in Chapter 5, where I highlight the need for repositories and general data search engines to leverage flexible, interlinked designs with varying levels of specificity. Partially in response to encouragement from search engine developers, i.e. Google Dataset Search (see Noy \& Benjelloun, 2020), many repositories are already taking on the work of describing data using general metadata schemas such as schema.org. ${ }^{30}$ This is an important step in facilitating the findability of data by search engines, but thought also needs to be given to how to continue to support or to distribute some of this work.

30 https://schema.org/ 


\section{Data supplies / metadata}

Index both disciplinary and multidisciplinary repositories

Index / point to data not created in academia

Work together to describe data using metadata which supports discovery by general data search systems

Engage with disciplinary communities and repository managers to improve metadata standardization

Implement automatic metadata enrichment techniques

Develop and implement metadata / documentation which includes common evaluation criteria

Develop layered metadata records (discovery, evaluation \& communication layers)

Develop spaces for co-evolution of metadata by both communities and repository managers

\section{Engagement / social interactions}

Identify communities of use at the repository level

Support ways to contact / interact with data creators

Integrate offline and online interactions around data, i.e. links to trainings or forums

Consider ranking by social signals

\section{Interface development / design}

Support keyword searching, browsing, APIs, graphical interfaces

Experiment with operationalizing principles, i.e. FAIR guidelines

Create differentiated search interfaces for user groups or based on level of expertise

Implement filters for common data uses

Experiment with visual overviews or maps of repository content

Implement tools supporting more specific search and sensemaking activities at the repository level

Rethink disciplinary categories for data within systems

Integrate data search tools with literature databases and data management tools

Box 9.1. Recommendations for systems designers and repository managers drawn from Chapters 4 and 5 . Items in italics could also be classified in the engagement / social interactions level.

The recommendations for metadata in the first section of Box 9.1 also draw on observations of the diverse ways which data, and metadata, are created and made available. Chapter 4 recommends that repository managers in particular engage with (disciplinary) communities to improve standardized metadata creation. While standardization is critical, both for discovery and evaluation, some of the recommendations advocate experimental ways of 
thinking about and implementing metadata. Chapter 5, for example, proposes a "layered" metadata model, which consists of three levels: general metadata to support discovery; more detailed metadata to support common evaluation and sensemaking criteria and activities; and a space for communication between data producers and consumers within the context of data themselves.

These communications could serve as the basis for identifying emergent terms and concepts for inclusion in schemas or for identifying local communities of use at the repository level, a recommendation presented in the second section of Box 9.1, engagement and social interactions. This section also summarizes other suggestions for facilitating the social interactions important in data discovery, such as providing mechanisms for exchanges between data creators and consumers, to integrate online and offline interactions around data and to consider ranking data results by social signals.

The final section of Box 9.1 includes recommendations for interface development and design; this section reflects the need for designs supporting adaptability and serendipity, as first discussed in Chapter 4. Discovery systems and repository interfaces should support a range of ways to discover data, i.e., keyword searching, graphical interfaces, browsing and APIs. Differentiated search interfaces or filters, i.e. for particular user groups, expertise levels or data uses, could also be implemented. In a discovery ecosystem linking repositories and general search engines, repositories face extra work to develop specialized features supporting both search and sensemaking which may not be offered by more general discovery tools.

Both repositories and general data discovery systems may need to rethink existing disciplinary categorizations for data to better account for the diversity of disciplines identified among researchers (Chapter 5) and the interdisciplinarity of research (Chapter 4). Finally, to support the interwoven nature of data discovery and other practices, data discovery tools could be linked with other systems, i.e. literature databases or data management tools, as proposed in Chapter 5.

\section{Recommendations to support data-centric sensemaking}

Chapter 7 proposes design recommendations to support data-centric sensemaking across three identified clusters of activities: inspecting data, engaging more deeply with content and placing data within broader contexts. Figure 9.1 summarizes these recommendations and places them in relation to contextual information and broader standards. 


\section{2 | Chapter 9}

Contextual information may be provided through a variety of formats, including metadata, README files or codebooks, and contain a range of details about data creation and documentation. Broader standards, which define common terms or abbreviations, experimental protocols or geographic names and locations, can be found, for example, in ontologies, thesauri or documented codes for best practice.

The recommendations for each cluster of sensemaking activities are provided the level of the data in Figure 9.1. Arrows are used to indicate where links between the data and contextual information/broader standards could be used to support these suggestions. Many of these recommendations are tailored to structured data, i.e. data in spreadsheet form, but could be extrapolated to other data as well. 


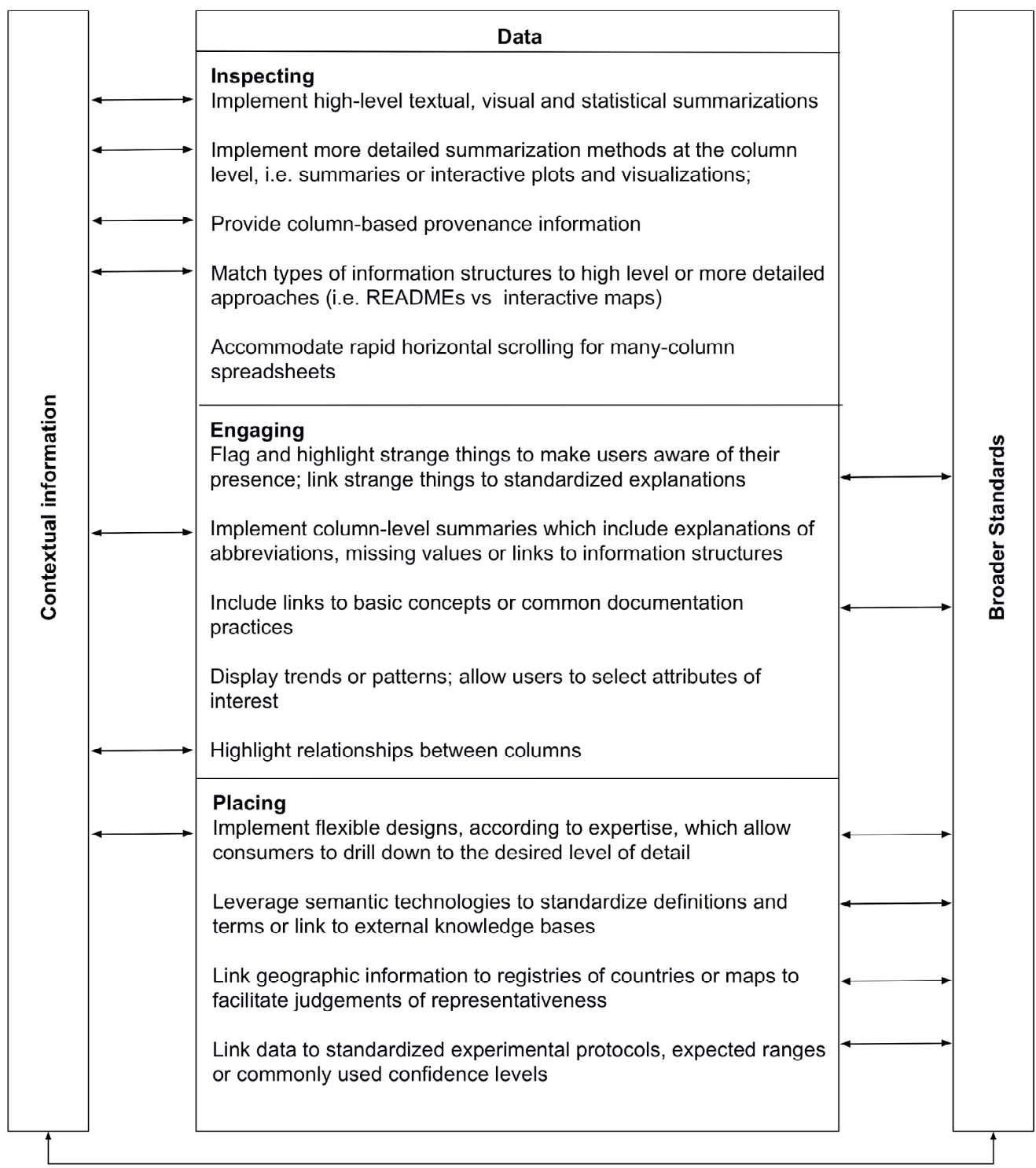

Figure 9.1. Recommendations for systems designers to support data-centric sensemaking activities. Arrows indicate where links to contextual information or broader standards are necessary.

Implementing high-level and more granular textual, visual or statistical summaries could be one way to facilitate inspecting activities. Column level summaries, drawing on relevant contextual information, could also describe the provenance of a column, i.e. how those data were collected or derived. Designers could also experiment with matching high-level 


\section{4 | Chapter 9}

inspecting activities to contextual information, such as README files, which provide a broad overview of the data.

Flagging and linking "strange things" within data to standard abbreviations or defined guidelines for best practices could aid researchers as they engage more deeply with data. Incorporating contextual information, column level summaries could also be helpful in explaining non-standard abbreviations or the meaning of documentation particular to the data, i.e. the handling of missing values. Patterns or trends which exist within the data themselves could also be displayed using interactive visualizations.

Designers could support placing activities by creating flexible designs, which allow researchers to drill down to necessary levels of detail to accommodate varying levels of expertise. Data could also be linked to external sources, such as maps, country registries, or standardized experimental protocols, to help researchers determine representativeness, i.e. of geographic elements within the data. Links to broader standards from relevant terms and concepts within contextual information could also help potential data users to place and understand data.

Although the majority of recommendations posed throughout the dissertation focus on systems development and design, I also suggest possible actions for other actors. Data creators, for example, could identify core, or anchor, variables to aid others in navigating their data. Data citation developers could consider implementing formats and standards which indicate how data have been used (Chapter 5) and document information about data provenance (Chapter 7 ).

\subsubsection{Policy recommendations}

In addition to having applications for design, this dissertation has the potential to inform policy development. Although there are an increasing number of policies regulating open data, data sharing and open science (see European Commission, 2019), there is often a mismatch between this discourse and the practices of various communities (Noorman et al., 2018). The following points, drawn from the entirety of work in this thesis, can aid the creation of policies reflective of actual practice.

\section{Data discovery and reuse are costly.}

Discovering and reusing data are costly practices for researchers; it takes time and effort to locate, understand and use data. Data discovery and reuse also involve infrastructural costs. 
Repository software and hardware must be purchased, developed or maintained. Archivists need to ensure that data are accurately described in standardized ways, and data must be stored for the long-term and be stably linked to contextual information and literature in order to be found and understood.

Policy makers should recognize the costs implicit in both discovering and reusing data. Funders could provide additional support for the often hidden work required by data repositories, i.e. metadata creation and experimentation, that is necessary to create a landscape integrating general and specific search systems and which supports various levels of sensemaking.

\section{Data discovery and reuse are situated within diverse communities.}

The word "community" is often used indiscriminately (Borgman, 2012) or to refer to disciplinary domains (see Wilkinson et al., 2016), although there is a great diversity of practice even within disciplines. Practices are also situated within other data communities, which form around common data, common data uses, or common research methodologies. Policies which equate communities with broad disciplinary domains may not be as relevant or sustainable as those taking a more nuanced perspective.

\section{Data discovery and reuse are not always relevant.}

Data discovery and reuse are not relevant for every research community or for every research question. The increased number of policies encouraging data sharing and reuse or of funding calls encouraging data science methodologies may privilege one way of conducting research. Similarly, as certain data become easier to seamlessly and automatically access, other data, those that are more challenging to access, may not be used as often. Policy makers should view data reuse as existing on a continuum and encourage a diversity of research approaches.

\section{Co-design of policies for data discovery and reuse}

Survey respondents in Chapter 5 perceive that their institutions support data sharing and reuse less than they or their colleagues do (Figure 5.2). This suggests a potential mismatch between the messages being communicated in institutional policies and their reception among researchers. Institutions could work to clarify policies or to increase outreach efforts. Another possibility would be to develop policies in conjunction with researchers, who may already have strong feelings about data sharing and reuse. 


\section{Flexible policies for data discovery and reuse}

Data discovery and reuse are not static or uniform practices. Relevant policies should allow for adaptation and flexibility. A layered approach to policy design, such as that described in (Cruz et al., 2019), provides one possibility. In this model, a general policy provides an overarching framework. More specific policies can be co-designed with communities to reflect norms and practices; these specific policies are then linked together under the umbrella of the broader framework. Data discovery and reuse are also evolving practices; policies should be living documents which are regularly revisited and easily modified.

\subsection{Valorization statement}

The synthesis and discussion presented in the previous section has the potential to inform the development of both systems and policy. There is also evidence that the work presented in this dissertation is already being taken up and applied within various areas of research.

The papers presented in Chapters 3 through 5 have been cited within a variety of journals and conference proceedings within information science (i.e. JASIST, the Journal of Documentation and the ACM Joint Conference on Digital Libraries); computer science (i.e. the VLDB Journal, the ACM SIGIR conference, and the Web conference); and STS (i.e. Big Data \& Society). ${ }^{31}$ It has also been referenced in outlets which cater to practitioners in library and information science, i.e. LIBER Quarterly and eLucidate, the journal of the UK electronic information group, as well as in doctoral dissertations and masters' theses. Citing authors are affiliated with institutions primarily across Europe and the United States, but also more globally, i.e. with institutions in Brazil and Malaysia.

The work has been cited in interdisciplinary (i.e. PLOS ONE) and discipline-specific journals (i.e. the Journal of Mechanical Design) in examinations of disciplinary practices of data discovery, sharing, analysis and management. It has also been referenced within the context of work focusing on systems development, data stewardship, linked open data and the development of FAIR metrics.

\footnotetext{
${ }^{31}$ Analysis was conducted using GoogleScholar on 14 October, 2020. The articles stemming from this dissertation have accrued a total of 52 citations, excluding self-citations, during the period of 2017 2020.
}

Some of these citations are to pre-prints of the published articles. However, It is likely that some of the observed citations stem from collaborations formed during the $\mathrm{PhD}$ thesis. 
This diversity reflects various engagements and collaborations developed over the course of four years. During this time, I have been an active member of the Data Discovery Paradigms Interest Group within the Research Data Alliance (Research Data Alliance, n.d.). Here, I was involved in a collaboration which resulted in a practical guide to help individuals locate data for research (Gregory, Khalsa, et al., 2018). This article, which has been downloaded over 2000 times, has since been integrated into online training material published by CESSDA, the Consortium for European Social Science Data Archives (CESSDA Training Team, 2020).

As a result of my research, I was invited to participate in various projects where data discovery was implicated, i.e. the Dagstuhl Perspectives Workshop on Implementing FAIR Data Infrastructures (Schloss Dagstuhl, 2018) and the GO FAIR implementation network focusing on semantic interoperability (GO Inter, n.d.). Building on this interest, a team of colleagues and I worked together to create a best practices guide for creating linked open data to enhance interoperability (Siebes et al., 2019), as part of the annual Mozilla-Library Carpentry Global Sprint series (Library Carpentry, 2019).

Citations and collaborations provide one way to think about the reach of this research; another is to consider how it has been integrated into the development of data search systems, particularly DataSearch, Elsevier's data search engine. I regularly presented the results of my research to the DataSearch team at internal project meetings as well as to Elsevier's broader research data management team. During these presentations, we discussed the recommendations summarized in Box 9.1, particularly suggestions to index diverse data supplies, to support social interactions and to rethink disciplinary classifications for data.

Since the beginning of this project in 2017, DataSearch has increased the diversity of data repositories which it indexes. It has also been integrated into an existing research data tool, Mendeley Data, and work is proceeding to link it with the Scopus literature database. Although considering how to support social interactions around data does not appear to be a high priority for development, assigning meaningful disciplinary classifications for data is a known problem that the design team is continuing to address. Although these changes align with some of my recommendations, it is difficult to say whether these developments were always planned, or whether my research directly influenced the design of the system.

This research used a theoretical framework developed, in part, to facilitate communication with systems designers. Although the recommendations made in Box 9.1 may not be realized in DataSearch (immediately), the communication of my findings to different 


\section{8 | Chapter 9}

stakeholders has been made easier using this approach. It has also become apparent that systems development and design is itself a sociotechnical process. It requires time and adjustment and is shaped by factors such as changes in organizational structure, staffing and competition - all of which influenced the DataSearch team at some point in the project.

The course of my research was also impacted by larger changes in the world, as my final year of work was conducted primarily during the COVID-19 pandemic. Fortunately, I had finished collecting data before the pandemic began. However, speaking engagements and new collaborations, such as a planned research stay at the Institute for Quantitative Social Sciences at Harvard University, were postponed or cancelled.

The collaborations, both realized and potential, that this research has stimulated are one of its most valuable outcomes. This research has brought together individuals with diverse expertise, motivations and approaches to studying data practices and for designing practical solutions to support them. Despite current global challenges, I remain optimistic that such collaborations will continue to stem from and build on this research, providing the opportunity to further trace the relation of data discovery and reuse with other scholarly practices, technologies, and communities. 
Recommendations and valorization | 279 

References 
282 
Adams, A., \& Blandford, A. (2005). Digital libraries' support for the user's "information journey." Proceedings of the 5th ACM/IEEE-CS Joint Conference on Digital Libraries - JCDL '05, 160. https://doi.org/10.1145/1065385.1065424

Agarwal, N. K. (2018). Exploring context in information behavior: Seeker, situation, surroundings, and shared identities. Morgan \& Claypool Publishers.

ah Kang, Y., \& Stasko, J. T. (2012). Examining the use of a visual analytics system for sensemaking tasks: Case studies with domain experts. IEEE Transactions on Visualization and Computer Graphics, 18(12), 2869-2878. https://doi.org/10.1109/TVCG.2012.224

Allen, M. (2017). Secondary data. In The SAGE Encyclopedia of Communication Research Methods. SAGE Publications, Inc. https://doi.org/10.4135/9781483381411

Allmendinger, J. (2015). Quests for interdisciplinarity: A challenge for the ERA and HORIZON 2020 (Policy Brief by the Research, Innovation, and Science Policy Experts (RISE) EUR 27370). https://ec.europa.eu/research/openvision/pdf/rise/allmendinger-interdisciplinarity.pdf

Aloia, N., Binding, C., Cuy, S., Doerr, M., Felicetti, A., Finn, J., Gavrilis, D., Nurra, F., Papatheodorou, C., \& Richards, J. (2017). Enabling European archaeological research: The ARIADNE e-Infrastructure. Internet Archaeology, 43, 1-21.

American Association for Public Opinion Research. (2016). Standard definitions: Final dispositions of case codes and outcome rates for surveys (9th ed.). American Association for Public Opinion Research.

Ames, D. P., Horsburgh, J. S., Cao, Y., Kadlec, J., Whiteaker, T., \& Valentine, D. (2012). HydroDesktop: Web services-based software for hydrologic data discovery, download, visualization, and analysis. Environmental Modelling and Software, 37, 146-156. https://doi.org/10.1016/j.envsoft.2012.03.013

Arbuckle, B. S., Kansa, S. W., Kansa, E., Orton, D., Çakirlar, C., Gourichon, L., Atici, L., Galik, A., Marciniak, A., Mulville, J., Buitenhuis, H., Carruthers, D., De Cupere, B., Demirergi, A., Frame, S., Helmer, D., Martin, L., Peters, J., Pöllath, N., ... Würtenberger, D. (2014). Data sharing reveals complexity in the westward spread of domestic animals across Neolithic Turkey. PLOS ONE, 9(6). https://doi.org/10.1371/journal.pone.0099845 
Atici, L., Birch, S. E. P., \& Erdoğu, B. (2017). Spread of domestic animals across Neolithic western Anatolia: New zooarchaeological evidence from Uğurlu Höyük, the island of Gökçeada, Turkey. PLOS ONE, 12(10), e0186519.

\section{https://doi.org/10.1371/journal.pone.0186519}

Atici, L., Kansa, S. W., Lev-Tov, J., \& Kansa, E. C. (2013). Other people's data: A demonstration of the imperative of publishing primary data. Journal of Archaeological Method and Theory, 20(4), 663-681. https://doi.org/10.1007/s10816-012-9132-9

Bahls, D., \& Tochtermann, K. (2013). Semantic retrieval interface for statistical research data. Proceedings of the 3rd International Workshop on Semantic Digital Archives, 1091, 93-103.

Baker, J., Jones, D. R., \& Burkman, J. (2009). Using visual representations of data to enhance sensemaking in data exploration task. Journal of the Association for Information Systems, 10(7), 2.

Baker, K. S., Duerr, R. E., \& Parsons, M. A. (2015). Scientific knowledge mobilization: Coevolution of data products and designated communities. International Journal of Digital Curation, 10(2), 110-135. https://doi.org/10.2218/ijdc.v10i2.346

Baker, K. S., \& Yarmey, L. (2009). Data stewardship: Environmental data curation and a web of repositories. International Journal of Digital Curation, 4(2), 12-27. https://doi.org/10.2218/ijdc.v4i2.90

Baker, M. (2016). 1,500 scientists lift the lid on reproducibility: Nature News \& Comment. https://www.nature.com/news/1-500-scientists-lift-the-lid-on-reproducibility-1.19970

Balog, K. (2018). Entity-oriented search (Vol. 39). Springer. https://doi.org/10.1007/978-3-31993935-3

Bates, M. (1989). The design of browsing and berrypicking techniques for the online search interface. Online Review, 13(5), 407-424.

Bates, M. (1990). Where should the person stop and the information search start? Information, Processing and Management, 26(5), 575-591. 
Beaulieu, A. (2004). From brainbank to database: The informational turn in the study of the brain. Studies in History and Philosophy of Biological and Biomedical Sciences, 35(2), 367-390. https://doi.org/10.1016/j.shpsc.2004.03.011

Bechtel, W. (2008). Mechanisms in cognitive psychology: What are the operations? Philosophy of Science, 75(5), 983-994. https://doi.org/10.1086/594540

Belkin, N. J. (1993). Interaction with texts: Information retrieval as information-seeking behavior. Information Retrieval, 55-66.

Belkin, N. J. (1996). Intelligent information retrieval: Whose intelligence? ISI '96: Proceedings of the Fifth International Symposium for Information Science, 25-31.

Benjelloun, O., Chen, S., \& Noy, N. (2020). Google Dataset Search by the numbers. ArXiv:2006.06894 [Cs]. http://arxiv.org/abs/2006.06894

Beran, B., Cox, S. J. D., Valentine, D., Zaslavsky, I., \& McGee, J. (2009). Web services solutions for hydrologic data access and cross-domain interoperability. International Journal on Advances in Intelligent Systems, $2(2 \& 3)$.

Berghmans, S., Cousijn, H., Deakin, G., Meijer, I., Mulligan, A., Plume, A., \& Waltman, L. (2017). Open data: The researcher perspective. Elsevier; Leiden University's Centre for Science and Technology Studies (CWTS). https://www.elsevier.com/about/open-science/researchdata/open-data-report

Bijker, W. E. (1993). Do not despair: There is life after constructivism. Science, Technology, \& Human Values, 18(1), 113-138. https://doi.org/10.1177/016224399301800107

Bijker, W. E. (1995). Sociohistorical technology studies. In S. Jasanoff, G. Markle, J. Peterson, \& T. Pinch (Eds.), Handbook of science and technology studies (pp. 229-256). SAGE Publications, Inc. https://doi.org/10.4135/9781412990127.n11

Bijker, W. E., \& Pinch, T. J. (1987). The social construction of facts and artefacts: Or how the sociology of science and the sociology of technology might benefit each other. In W. E. Bijker, T. P. Hughes, \& T. J. Pinch (Eds.), The social construction of technological systems: New directions in sociology and history of technology. MIT Press.

Bilder, C. R., \& Loughin, T. M. (2015). Analysis of categorical data with R. CRC Press. 
Birnholtz, J. P., \& Bietz, M. J. (2003). Data at work: Supporting sharing in science and engineering. In K. Schmidt, M. Pendergast, M. Tremaine, \& C. Simone (Eds.), Proceedings of the 2003 International ACM SIGGROUP Conference on Supporting Group Work, GROUP 2003 (pp. 339-348). ACM. https://doi.org/10.1145/958160.958215

Blandford, A., \& Attfield, S. (2010). Interacting with information. Morgan \& Claypool Publishers. https://doi.org/10.2200/S00227ED1V01Y200911HCI006

Bloor, D. (1991). Knowledge and social imagery (2nd ed.). University of Chicago Press.

Borgman, C. L. (2012). The conundrum of sharing research data. Journal of the American Society for Information Science and Technology, 63(6), 1059-1078.

https://doi.org/10.1002/asi.22634

Borgman, C. L. (2015a). Big data, little data, no data: Scholarship in the networked world. MIT Press.

Borgman, C. L. (2015b). If data sharing is the answer, what is the question? ERCIM NEWS, 100, $15-16$.

Borgman, C. L. (2016). Data citation as a bibliometric oxymoron. In C. R. Sugimoto (Ed.), Theories of informetrics and scholarly communication (pp. 93-116). De Gruyter. https://doi.org/10.1515/9783110308464-008

Borgman, C. L. (2019). The lives and after lives of data. Harvard Data Science Review, 1(1). https://doi.org/10.1162/99608f92.9a36bdb6

Borgman, C. L., Darch, P. T., Sands, A. E., \& Golshan, M. S. (2016). The durability and fragility of knowledge infrastructures: Lessons learned from astronomy. Proceedings of the Association for Information Science and Technology, 53, 1-10.

https://doi.org/10.1002/pra2.2016.14505301057

Borgman, C. L., Darch, P. T., Sands, A. E., Pasquetto, I. V., Golshan, M. S., Wallis, J. C., \& Traweek, S. (2015). Knowledge infrastructures in science: Data, diversity, and digital libraries. International Journal on Digital Libraries, 16(3-4), 207-227.

https://doi.org/10.1007/s00799-015-0157-z 
Borgman, C. L., Hirsh, S. G., \& Hiller, J. (1996). Rethinking online monitoring methods for information retrieval systems: From search product to search process. Journal of the American Society for Information Science, 47(7), 568-583.

Borgman, C. L., Scharnhorst, A., \& Golshan, M. S. (2018). Digital data archives as knowledge infrastructures: Mediating data sharing and reuse. ArXiv:1802.02689 [Cs]. http://arxiv.org/abs/1802.02689

Borgman, C. L., Scharnhorst, A., \& Golshan, M. S. (2019). Digital data archives as knowledge infrastructures: Mediating data sharing and reuse. Journal of the Association for Information Science and Technology, 70(8), 888-904. https://doi.org/10.1002/asi.24172

Borgman, C. L., Smart, L. J., Millwood, K. A., Finley, J. R., Champeny, L., Gilliland, A. J., \& Leazer, G. H. (2005). Comparing faculty information seeking in teaching and research: Implications for the design of digital libraries. Journal of the American Society for Information Science and Technology, 56(6), 636-657. https://doi.org/10.1002/asi.20154

Borgman, C. L., Van de Sompel, H., Scharnhorst, A., van den Berg, H., \& Treloar, A. (2015). Who uses the digital data archive? An exploratory study of DANS. Proceedings of the Association for Information Science and Technology, 52, 1-5.

https://doi.org/10.1002/pra2.2015.145052010096

Borgman, C. L., Wallis, J. C., \& Enyedy, N. (2007). Little science confronts the data deluge: Habitat ecology, embedded sensor networks, and digital libraries. International Journal on Digital Libraries, 7(1-2), 17-30. https://doi.org/10.1007/s00799-007-0022-9

Borgman, C. L., Wallis, J. C., Mayernik, M. S., \& Pepe, A. (2007). Drowning in data: Digital library architecture to support scientific use of embedded sensor networks. Proceedings of the 7th ACM/IEEE-CS Joint Conference on Digital Libraries, 269-277.

https://doi.org/10.1145/1255175.1255228

Borgman, C. L., Wofford, M. F., Golshan, M. S., Darch, P. T., \& Scroggins, M. J. (2019). Collaborative ethnography at scale: Reflections on 20 years of data integration. [preprint]. https://escholarship.org/uc/item/5bb8b1tn\#article main 
Borgman, C. L., Wofford, M. F., Golshan, M. S., Darch, P. T., \& Scroggins, M. J. (2020).

Collaborative ethnography at scale: Reflections on 20 years of data integration. [revised preprint]. https://escholarship.org/uc/item/5bb8b1tn\#article main

Börner, K., \& Record, E. (2017). Macroscopes for making sense of science. Proceedings of the Practice and Experience in Advanced Research Computing 2017 on Sustainability, Success and Impact, article no. 64.

Boukhelifa, N., Perrin, M.-E., Huron, S., \& Eagan, J. (2017). How data workers cope with uncertainty: A task characterisation study. Proceedings of the $2017 \mathrm{CHI}$ Conference on Human Factors in Computing Systems, 3645-3656.

https://doi.org/10.1145/3025453.3025738

Bowker, G. C. (2000a). Biodiversity datadiversity. Social Studies of Science, 30(5), 643-683.

Bowker, G. C. (2000b). Work and information practices in the sciences of biodiversity. Proceedings of the 26th International Conference on Very Large Data Bases.

Bowker, G. C., \& Star, S. L. (1999). Sorting things out: Classification and its consequences. The MIT Press.

Bradford, S. C. (1950). Documentation. Public Affairs Press.

Broom, A., Cheshire, L., \& Emmison, M. (2009). Qualitative researchers' understandings of their practice and the implications for data archiving and sharing. Sociology, 43(6), 1163-1180. https://doi.org/10.1177/0038038509345704

Brown, C. (2003). The changing face of scientific discourse: Analysis of genomic and proteomic database usage and acceptance. Journal of the American Society for Information Science and Technology, 54(10), 926-938. https://doi.org/10.1002/asi.10289

Buckland, M. K. (1991). Information as thing. Journal of the American Society for Information Science, 42(5), 351-360. https://doi.org/10.1002/(SICI)1097-4571(199106)42:5<351::AID-

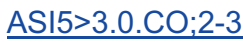

Burnett, G., Burnett, K., Kazmer, M. M., Marty, P. F., Worrall, A., Knop, B., Hinnant, C. C., Stvilia, B., \& Wu, S. (2014). Don't tap on the glass, you'll anger the fish! The information worlds of 
distributed scientific teams. In H. Rosenbaum \& P. Fichman (Eds.), Social informatics: Past, present and future (pp. 118-133). Cambridge Scholars Publishing.

Cafarella, M. J., Halevy, A., \& Madhavan, J. (2011). Structured data on the web. Communications of the ACM, 54(2), 72-79. https://doi.org/10.1145/1897816.1897839

Callaghan, S., Donegan, S., Pepler, S., Thorley, M., Cunningham, N., Kirsch, P., Ault, L., Bell, P., Bowie, R., Leadbetter, A., Lowry, R., Moncoiffé, G., Harrison, K., Smith-Haddon, B., Weatherby, A., \& Wright, D. (2012). Making data a first class scientific output: Data citation and publication by NERC's environmental data centres. International Journal of Digital Curation, 7(1), 107-113. https://doi.org/10.2218/ijdc.v7i1.218

Candela, L., Castelli, D., Manghi, P., \& Tani, A. (2015). Data journals: A survey. Journal of the Association for Information Science and Technology, 66(9), 1747-1762. https://doi.org/10.1002/asi.23358

Carlson, J., \& Stowel-Bracke, M. (2013). Data management and sharing from the perspective of graduate students: An examination of the culture and practice at the water quality field station. Libraries Faculty and Staff Scholarship and Research, 13(4), 343-361. https://doi.org/10.1353/pla.2013.0034

Case, D. O., \& Given, L. M. (2016). Looking for information: A survey of research on information seeking, needs, and behavior (Fourth edition). Emerald Group Publishing Limited.

Cash, D. W., Clark, W. C., Alcock, F., Dickson, N. M., Eckley, N., Guston, D. H., Jäger, J., \& Mitchell, R. B. (2003). Knowledge systems for sustainable development. Proceedings of the National Academy of Sciences of the United States of America, 100(14), 8086-8091. https://doi.org/10.1073/pnas.1231332100

CESSDA Training Team. (2020). CESSDA Data Management Expert Guide. Zenodo. https://zenodo.org/record/3820473

Chapman, A., Simperl, E., Koesten, L., Konstantinidis, G., Ibáñez, L.-D., Kacprzak, E., \& Groth, P. (2020). Dataset search: A survey. The VLDB Journal, 29(1), 251-272. https://doi.org/10.1007/s00778-019-00564-x 
Chatman, E. A. (1999). A theory of life in the round. Journal of the American Society for Information Science, 50(3), 207-217. https://doi.org/10.1002/(SICl)10974571(1999)50:3<207::AID-ASI3>3.0.CO;2-8

Chin, G., Jr., \& Lansing, C. S. (2004). Capturing and supporting contexts for scientific data sharing via the biological sciences collaboratory. Proceedings of the 2004 ACM Conference on Computer Supported Cooperative Work, 409-418.

https://doi.org/10.1145/1031607.1031677

Choo, C. W. (2007). Information seeking in organizations: Epistemic contexts and contests. Information Research, 12(2), 15.

Choudhury, S., Fishman, J. R., McGowan, M. L., \& Juengst, E. T. (2014). Big data, open science and the brain: Lessons learned from genomics. Frontiers in Human Neuroscience, 8, 1-10. https://doi.org/10.3389/fnhum.2014.00239

Clough, P. D. D., Sedghi, S., \& Sanderson, M. (2008). A study on the relevance criteria for medical images. Pattern Recognition Letters, 29(15), 2046-2057. https://doi.org/10.1186/1742-7622-5-2

CODATA. (n.d.). Decadal programme: Making data work for cross-domain grand challenges. Retrieved July 20, 2020, from https://codata.org/initiatives/strategic-programme/decadalprogramme/

Cooper, D., \& Springer, R. (2019). Data communities: A new model for supporting STEM data sharing. Ithaka S+R. https://doi.org/10.18665/sr.311396

Cornell University Research Data Management Service Group. (n.d.). Guide to writing "readme" style metadata. Guide to Writing "Readme" Style Metadata. Retrieved October 19, 2020, from https://data.research.cornell.edu/content/readme

Corti, L. (2007). Re-using archived qualitative data-Where, how, why? Archival Science, 7(1), 37-54. https://doi.org/10.1007/s10502-006-9038-y

Courtright, C. (2007). Context in information behavior research. Annual Review of Information Science and Technology, 41(1), 273-306. https://doi.org/10.1002/aris.2007.1440410113 
Cowan, R. S. (1987). The consumption junction: A proposal for research strategies in the sociology of technology. In W. E. Bijker, T. P. Hughes, \& T. J. Pinch (Eds.), The social construction of technological systems: New directions in the sociology and history of technology. MIT Press.

Cox, A. M., \& Wan Ting Tam, W. (2018). A critical analysis of lifecycle models of the research process and research data management. Aslib Journal of Information Management, 70(2), 142-157. https://doi.org/10.1108/AJIM-11-2017-0251

Cragin, M. H., Chao, T. C., \& Palmer, C. L. (2011). Units of evidence for analyzing subdisciplinary difference in data practice studies. Proceedings of the 11th ACM/IEEE-CS Joint Conference on Digital Libraries. https://doi.org/10.1145/1998076.1998175

Crestani, F., \& Du, H. (2006). Written versus spoken queries: A qualitative and quantitative comparative analysis. Journal of the American Society for Information Science and Technology, 57(7), 881-890. https://doi.org/10.1002/asi.20350

Cruz, M., Dintzner, N., Dunning, A., Kuil, A. van der, Plomp, E., Teperek, M., Velden, Y. T. der, \& Versteeg, A. (2019). Policy needs to go hand in hand with practice: The learning and listening approach to data management. Data Science Journal, 18(1), 45. https://doi.org/10.5334/dsj-2019-045

Curty, R. (2016). Factors influencing research data reuse in the social sciences: An exploratory study. International Journal of Digital Curation, 11(1), 96-117. https://doi.org/10.2218/ijdc.v11i1.401

Curty, R., Yoon, A., Jeng, W., \& Qin, J. (2016). Untangling data sharing and reuse in social sciences. Proceedings of the Association for Information Science and Technology, 53, 1-5. https://doi.org/10.1002/pra2.2016.14505301025

Dallmeier-Tiessen, S., \& Mele, S. (2014). Integrating data in the scholarly record: Communitydriven digital libraries in high-energy physics. Zeitschrift Fur Bibliothekswesen Und Bibliographie, 61(4-5), 220-223. https://doi.org/10.3196/1864295014614574 
DataCite Metadata Working Group. (2019). DataCite Metadata Schema documentation for the publication and citation of research data v4.3 (4.3, p. 73 pages) [Application/pdf]. DataCite. https://schema.datacite.org/meta/kernel-4.3/

Davis, M. L. E. S., Tenopir, C., Allar, S., \& Frame, M. T. (2014). Facilitating access to biodiversity information: A survey of users' needs and practices. Environmental Management, 53(3), 690-701. https://doi.org/10.1007/s00267-014-0229-7

de Vaus, D. (2013). Surveys In Social Research. Routledge.

De-Arteaga, M., Eggel, I., Do, B., Rubin, D., Kahn, C. E., \& Müller, H. (2015). Comparing image search behavior in the ARRS GoldMiner search engine and a clinical PACS/RIS. Journal of Biomedical Informatics, 56, 57-64. https://doi.org/10.1016/j.jbi.2015.04.013

Dervin, B. (1983). An overview of sense-making: Concepts, methods, and results to date. Annual Meeting of International Communication Association, Dallas. https://www.ideals.illinois.edu/bitstream/handle/2142/2281/Dervin83a.htm

Dervin, B. (1992). From the mind's eye of the user: The sense-making qualitative-quantitative methodology. In J. D. Glazier \& R. R. Powell (Eds.), Qualitative Research in Information Management (pp. 61-84). Libraries Unlimited. https://ci.nii.ac.jp/naid/10020996723/

Dervin, B. (1997). Given a context by any other name: Methodological tools for taming the unruly beast. In P. Vakkari, R. Savolainen, \& B. Dervin (Eds.), Information seeking in context (pp. 13-38). Taylor Graham.

Dervin, B. (1998). Sense-making theory and practice: An overview of user interests in knowledge seeking and use. Journal of Knowledge Management, 2(2), 36-46.

Dervin, B., \& Nilan, M. (1986). Information needs and uses. Annual Review of Information Science and Technology, 21, 3-33.

Devarakonda, R., Palanisamy, G., Green, J. M., \& Wilson, B. E. (2011). Data sharing and retrieval using OAI-PMH. Earth Science Informatics, 4(1), 1-5. https://doi.org/10.1007/s12145-010-0073-0

Digital Curation Centre. (n.d.). Overview of funders' data policies. Retrieved June 28, 2018, from http://www.dcc.ac.uk/resources/policy-and-legal/overview-funders-data-policies. 
Digital Science, Fane, B., Ayris, P., Hahnel, M., Hrynaszkiewicz, I., Baynes, G., \& Farrell, E. (2019). The state of open data report 2019 [Report]. Digital Science.

https://doi.org/10.6084/m9.figshare.9980783.v2

Digital Science, Hahnel, M., Fane, B., Treadway, J., Baynes, G., Wilkinson, R., Mons, B., Schultes, E., Olavo Bonino da Silva Santos, L., Arefiev, P., \& Osipov, I. (2018). The state of open data report 2018.

https://digitalscience.figshare.com/articles/The State of Open Data Report 2018/7195058

Dillo, I., \& Doorn, P. (2014). The front office-back office model: Supporting research data management in the Netherlands. International Journal of Digital Curation, 9(2), 39-46. https://doi.org/10.2218/ijdc.v9i2.333

Doorn, P., Dijk, E., \& Grootveld, M. (2017, September). FAIR metrics—Starring your data sets: A lightweight FAIR data assessment tool. Open Science FAIR, Athens, Greece. https://eoscpilot.eu/content/osfair-2017-fair-metrics---starring-your-data-sets-lightweight-fairdata-assessment-tool

Doorn, P. (2020). Archiving and managing research data. Archivar, 73(01), 44-50.

Dourish, P. (2004). What we talk about when we talk about context. Personal and Ubiquitous Computing, 8(1), 19-30. https://doi.org/10.1007/s00779-003-0253-8

Dow, A. K., Dow, E. M., Fitzsimmons, T. D., \& Materise, M. M. (2015). Harnessing the environmental data flood: A comparative analysis of hydrologic, oceanographic, and meteorological informatics platforms. Bulletin of the American Meteorological Society, 96(5), 725-736. https://doi.org/10.1175/BAMS-D-13-00178.1

Dulisch, N., Kempf, A. O., \& Schaer, P. (2015). Query expansion for survey question retrieval in the social sciences. In S. Kapidakis, C. Mazurek, \& M. Werla (Eds.), Research and advanced technology for digital libraries. (Vol. 9316, pp. 28-39). Springer, Cham. https://doi.org/10.1007/978-3-319-24592-8 3

Dunn, O. J. (1961). Multiple comparisons among means. Journal of the American Statistical Association, 56(293), 52-64. https://doi.org/10.1080/01621459.1961.10482090 
Edwards, P. N. (2010). A vast machine: Computer models, climate data, and the politics of global warming. MIT Press. https://mitpress.mit.edu/books/vast-machine

Edwards, P. N., Mayernik, M. S., Batcheller, A. L., Bowker, G. C., \& Borgman, C. L. (2011). Science friction: Data, metadata, and collaboration. Social Studies of Science, 41(5), 667690. https://doi.org/10.1177/0306312711413314

Ellis, D., Cox, D., \& Hall, K. (1993). A comparison of the information seeking patterns of researchers in the physical and social sciences. Journal of Documentation, 49(4), 356-369. https://doi.org/10.1108/eb026919

Ellis, D., \& Haugan, M. (1997). Modelling the information seeking patterns of engineers and research scientists in an industrial environment. Journal of Documentation, 53(4), 384-403. https://doi.org/10.1108/EUM0000000007204

Elsevier. (2020). Scopus content coverage guide. Amsterdam: Elsevier BV. https://www.elsevier.com/ data/assets/pdf file/0007/69451/0597-Scopus-Content-

\section{Coverage-Guide-US-LETTER-v4-HI-singles-no-ticks.pdf}

Eppler, M. J., \& Mengis, J. (2004). The concept of information overload-a review of literature from organization science, accounting, marketing, MIS, and related disciplines. The Information Society, 20(5), 325-344. https://doi.org/10.1080/01972240490507974

Erinjeri, J. P., Picus, D., Prior, F. W., Rubin, D. A., \& Koppel, P. (2009). Development of a Google-based search engine for data mining radiology reports. Journal of Digital Imaging, 22(4), 348-356. https://doi.org/10.1007/s10278-008-9110-7

European Commission. (2017). EOSC declaration: European Open Science Cloud: New research \& innovation opportunities. https://ec.europa.eu/research/openscience/index.cfm?pg=openscience-cloud

European Commission. (2018a). Cost-benefit analysis for FAIR research data: Cost of not having FAIR research data. https://op.europa.eu/en/publication-detail/-/publication/d375368c-1a0a11e9-8d04-01aa75ed71a1

European Commission. (2018b). Ethics \& data protection. 
European Commission. (2018c). Turning FAIR into reality. Final report and action plan from the European Commission Expert Group on FAIR data. European Commission. Directorate General for Research and Innovation. Directorate B Open Innovation and Open Science. Unit B2 Open Science. https://ec.europa.eu/info/sites/info/files/turning fair into reality 1.pdf European Commission. (2019). Facts and figures for open research data [Text]. https://ec.europa.eu/info/research-and-innovation/strategy/goals-research-and-innovationpolicy/open-science/open-science-monitor/facts-and-figures-open-research-data en

Faniel, I. M., Barrera-Gomez, J., Kriesberg, A., \& Yakel, E. (2013). A comparative study of data reuse among quantitative social scientists and archaeologists. IConference 2013 Proceedings, 797-800. https://doi.org/10.9776/13391

Faniel, I. M., Frank, R. D., \& Yakel, E. (2019). Context from the data reuser's point of view. Journal of Documentation, 75(6), 1274-1297. https://doi.org/10.1108/JD-08-2018-0133

Faniel, I. M., Kansa, E., Kansa, S. W., Barrera-Gomez, J., \& Yakel, E. (2013). The challenges of digging data: A study of context in archaeological data reuse. JCDL '13: Proceedings of the 13th ACM/IEEE-CS Joint Conference on Digital Libraries, 295-304.

https://doi.org/10.1145/2467696.2467712

Faniel, I. M., Kriesberg, A., \& Yakel, E. (2012). Data reuse and sensemaking among novice social scientists. Proceedings of the American Society for Information Science and Technology, 49, 1-10. https://doi.org/10.1002/meet.14504901068

Faniel, I. M., Kriesberg, A., \& Yakel, E. (2016). Social scientists' satisfaction with data reuse. Journal of the Association for Information Science and Technology, 67(6), 1404-1416. https://doi.org/10.1002/asi.23480

Faniel, I. M., \& Yakel, E. (2017). Practices do not make perfect: Disciplinary data sharing and reuse practices and their implications for repository data curation. In Curating research data: Practical strategies for your digital repository (Vol. 1, pp. 103-126). Association of College and Research Libraries. https://www.oclc.org/research/publications/2017/practices-do-not$\underline{\text { make-perfect.html }}$ 
Faniel, I. M., \& Zimmerman, A. (2011). Beyond the data deluge: A research agenda for largescale data sharing and reuse. International Journal of Digital Curation, 6(1), 58-69. https://doi.org/10.2218/ijdc.v6i1.172

Fear, K. (2013). Measuring and anticipating the impact of data reuse [University of Michigan]. https://deepblue.lib.umich.edu/handle/2027.42/102481

Fielding, N. G., \& Fielding, J. L. (2008). Resistance and adaptation to criminal identity: Using secondary analysis to evaluate classic studies of crime and deviance. Historical Social Research, 33(3), 75-93. https://doi.org/10.1177/S0038038500000419

Fiore, S. M., Cuevas, H. M., \& Oser, R. L. (2003). A picture is worth a thousand connections: The facilitative effects of diagrams on mental model development and task performance. Computers in Human Behavior, 19(2), 185-199. https://doi.org/10.1016/S0747$\underline{5632(02) 00054-7}$

Firestone, W. (1993). Alternative arguments for generalizing from data as applied to qualitative research. Educational Researcher, 22(4), 16-23.

\section{https://doi.org/10.3102/0013189X022004016}

Floridi, L. (2010). Information: A very short introduction (Issue Vol. 225). Oxford University Press.

Freund, L. (2013). A cross-domain analysis of task and genre effects on perceptions of usefulness. Information Processing \& Management, 49(5), 1108-1121. https://doi.org/10.1016/j.ipm.2012.08.007

Furnas, G. W., \& Russell, D. M. (2005). Making sense of sensemaking. Extended Abstracts Proceedings of the 2005 Conference on Human Factors in Computing Systems, CHI 2005, 2115-2116. https://doi.org/10.1145/1056808.1057113

Gamma, E. (1995). Design patterns: Elements of reusable object-oriented software. Pearson Education India.

Gangemi, A., \& Presutti, V. (2009). Ontology design patterns. In S. Staab \& R. Studer (Eds.), Handbook on Ontologies (pp. 221-243). Springer. https://doi.org/10.1007/978-3-540-92673$\underline{310}$ 
Garnett, E. (2017). Air pollution in the making: Multiplicity and difference in interdisciplinary data practices. Science Technology and Human Values.

\section{https://doi.org/10.1177/0162243917699974}

Geertz, C. (1973). Thick description: Toward an interpretive theory of culture. In The interpretation of cultures: Selected essays (Vol. 3, pp. 1-33). Basic Books.

Gil, Y., David, C. H., Demir, I., Essawy, B. T., Fulweiler, R. W., Goodall, J. L., Karlstrom, L., Lee, H., Mills, H. J., Oh, J.-H., Pierce, S. A., Pope, A., Tzeng, M. W., Villamizar, S. R., \& Yu, X. (2016). Toward the geoscience paper of the future: Best practices for documenting and sharing research from data to software to provenance. Earth and Space Science, 3(10), 388-415. https://doi.org/10.1002/2015EA000136

GO Inter. (n.d.). GO FAIR. Retrieved October 14, 2020, from https://www.gofair.org/implementation-networks/overview/go-inter/

Granlund, A., Lafrenière, D., \& Carr, D. A. (2001). A pattern-supported approach to the user interface design process. International Conference on Human-Computer Interaction.

Gray, A. J. G., Goble, C., \& Jimenez, R. C. (2017). From potato salad to protein annotation. International Semantic Web Conference, 4. https://bioschemas.org

Gray, J. (2009). Jim Gray on eScience: A transformed scientific method. In T. Hey, S. Tansley, \& K. Tolle (Eds.), The fourth paradigm: Data-intensive scientific discovery (pp. xvii-xxxi). Microsoft Research. https://www.microsoft.com/en-us/research/publication/fourth-paradigmdata-intensive-scientific-discovery/.

Gregory, K. (2020a). Data discovery and reuse practices in research [dataset]. Data Archiving and Networked Services (DANS). https://doi.org/10.17026/dans-xsw-kkeq

Gregory, K. (2020b). A dataset describing data discovery and reuse practices in research. Scientific Data, 7(232). https://doi.org/10.1038/s41597-020-0569-5

Gregory, K., Cousijn, H., Groth, P., Scharnhorst, A., \& Wyatt, S. (2018). Understanding data retrieval practices: A social informatics perspective. [preprint]. arXiv:1801.04971. 
Gregory, K., Cousijn, H., Groth, P., Scharnhorst, A., \& Wyatt, S. (2019). Understanding data search as a socio-technical practice. Journal of Information Science.

https://doi.org/10.1177/0165551519837182

Gregory, K., Groth, P., Cousijn, H., Scharnhorst, A., \& Wyatt, S. (2019). Searching data: A review of observational data retrieval practices in selected disciplines. Journal of the Association for Information Science and Technology, 70, 419-432. https://doi.org/10.1002/asi.24165

Gregory, K., Groth, P., Scharnhorst, A., \& Wyatt, S. (2020). Lost or Found? Discovering data needed for research. Harvard Data Science Review.

\section{https://doi.org/10.1162/99608f92.e38165eb}

Gregory, K., Khalsa, S. J., Michener, W. K., Psomopoulos, F. E., de Waard, A., \& Wu, M. (2018). Eleven quick tips for finding research data. PLOS Computational Biology, 14(4), e1006038. https://doi.org/10.1371/journal.pcbi.1006038

Groth, P., Cousijn, H., Clark, T., \& Goble, C. (2020). FAIR Data Reuse - The path through data citation. Data Intelligence, 78-86. https://doi.org/10.1162/dint a 00030

Groth, P., Koesten, L., Mayr, P., de Rijke, M., \& Simperl, E. (2018). DATA SEARCH'18Searching data on the web. ArXiv:1805.11883 [Cs]. http://arxiv.org/abs/1805.11883

Hand, M., Shove, E., \& Southerton, D. (2005). Explaining showering: A discussion of the material, conventional, and temporal dimensions of practice. Sociological Research Online, 10(2), 113. https://doi.org/10.5153/sro.1100

Harzing, A.-W. (2006). Response styles in cross-national survey research: A 26-country study. International Journal of Cross Cultural Management, 6(2), 243-266.

\section{https://doi.org/10.1177/1470595806066332}

Hearst, M. (2009). Search user interfaces. Cambridge University Press.

Heckel, F., Arlt, F., Geisler, B., Zidowitz, S., \& Neumuth, T. (2016). Evaluation of image quality of MRI data for brain tumor surgery. Proc. SPIE 9787, Medical Imaging 2016: Image Perception, Observer Performance, and Technology Assessment, 9787. https://doi.org/10.1117/12.2214944 
Hersh, W., Müller, H., Gorman, P., \& Jensen, J. (2005). Task analysis for evaluating image retrieval systems in the ImageCLEF biomedical image retrieval task. Slice of Life Conference on Multimedia in Medical Education.

Hidi, S., \& Anderson, V. (1986). Producing written summaries: Task demands, cognitive operations, and implications for instruction. Review of Educational Research, 56(4), 473493.

Hine, C. (2007). Multi-sited ethnography as a middle range methodology for contemporary STS. Science, Technology, \& Human Values, 32(6), 21.

Hoeppe, G. (2014). Working data together: The accountability and reflexivity of digital astronomical practice. Social Studies of Science, 44(2), 243-270. https://doi.org/10.1177/0306312713509705

Holland, S., Hosny, A., Newman, S., Joseph, J., \& Chmielinski, K. (2018). The dataset nutrition label: A framework to drive higher data quality standards. CoRR, abs/1805.03677. http://arxiv.org/abs/1805.03677

Holtzblatt, K., \& Beyer, H. (2017). User data drives design. In K. Holtzblatt \& H. Beyer (Eds.), Contextual design (2nd ed., pp. 31-41). Morgan Kaufmann. https://doi.org/10.1016/B978-0$\underline{12-800894-2.00002-8}$

Honor, L. B., Haselgrove, C., Frazier, J. A. J. A., \& Kennedy, D. N. D. N. (2016). Data citation in neuroimaging: Proposed best practices for data identification and attribution. Frontiers in Neuroinformatics, 10(AUG), 1-12. https://doi.org/10.3389/fninf.2016.00034 Ingwersen, P. (1992). Information retrieval interaction. Taylor Graham. Ingwersen, P. (1996). Cognitive perspectives of information retrieval interaction: Elements of a cognitive IR theory. Journal of Documentation, 52(1), 3-50.

Janse, G. (2006). Information search behavior of European forest policy decision-makers. Forest Policy and Economics, 8(6), 579-592.

Jansen, B. J., \& Rieh, S. Y. (2010). The seventeen theoretical constructs of information searching and information retrieval. Journal of the American Society for Information Science and Technology, n/a-n/a. https://doi.org/10.1002/asi.21358 
Järvelin, K., \& Wilson, T. D. (2003). On conceptual models for information seeking and retrieval research. Information Research, 9(1), 1-23.

Jirotka, M., Procter, R., Hartswood, M., Slack, R., Simpson, A., Coopmans, C., Hinds, C., \& Voss, A. (2005). Collaboration and trust in healthcare innovation: The eDiaMoND case study. Computer Supported Cooperative Work, 14(4), 369-398. https://doi.org/10.1007/s10606$\underline{005-9001-0}$

Jisc. (2013). How Jisc is helping researchers: Research lifecycle diagram. https://webarchive.nationalarchives.gov.uk/20140702225057/http://www.jisc.ac.uk/whatwedo /campaigns/res3/jischelp.aspx\#simulate

Julien, H., \& Duggan, L. J. (2000). A longitudinal analysis of the information needs and uses literature. Library \& Information Science Research, 22(3), 291-309. https://doi.org/10.1016/S0740-8188(99)00057-2

Kacprzak, E., Koesten, L. M., Ibáñez, L.-D., Simperl, E., \& Tennison, J. (2017). A query log analysis of dataset search. In J. Cabot, R. De Virgilio, \& R. Torlone (Eds.), Web Engineering. ICWE 2017. (Vol. 10360, pp. 429-436). Springer, Cham.

Kalpathy-Cramer, J., de Herrera, A. G. S., Demner-Fushman, D., Antani, S., Bedrick, S., \& Müller, H. (2015). Evaluating performance of biomedical image retrieval systems - an overview of the medical image retrieval task at ImageCLEF 2004-2013. Computerized Medical Imaging and Graphics, 39, 55-61.

https://doi.org/10.1016/j.compmedimag.2014.03.004

Karcher, S., Kirilova, D., \& Weber, N. (2016). Beyond the matrix: Repository services for qualitative data. IFLA Journal, 42(4). https://doi.org/10.1177/0340035216672870

Kelly, D. (2009). Methods for evaluating interactive information retrieval systems with users. Foundations and Trends in Information Retrieval, 3(1-2), 1-224. https://doi.org/10.1561/1500000012

Kennan, M. A., \& Markauskaite, L. (2015). Research data management practices: A snapshot in time. International Journal of Digital Curation, 10(2), 69-95.

https://doi.org/10.2218/ijdc.v10i2.329 
Kern, D., \& Mathiak, B. (2015). Are there any differences in data set retrieval compared to wellknown literature retrieval? In S. Kapidakis, C. Mazurek, \& M. Werla (Eds.), Research and advanced technology for digital libraries (Vol. 9316, pp. 197-208). Springer, Cham. https://doi.org/10.1007/978-3-319-24592-8 15

Key Perspectives. (2010). Data dimensions: Disciplinary differences in research data sharing, reuse and long term viability. SCARP Synthesis Study. Digital Curation Centre. http://www.dcc.ac.uk/scarp

Khan, A., Tiropanis, T., \& Martin, D. (2016). Exploiting semantic annotation of content with Linked Open Data (LoD) to improve searching performance in web repositories of multi-disciplinary research data. In Braslavski P. et al. (Ed.), Information Retrieval. RuSSIR 2015. Communications in Computer and Information Science (Vol. 573, pp. 130-145).

Kim, J. (2020). An analysis of data paper templates and guidelines: Types of contextual information described by data journals. Science Editing, 7(1), 16-23.

\section{https://doi.org/10.6087/kcse.185}

Kim, S., \& Gilbertson, J. (2007). Information requirements of cancer center researchers focusing on human biological samples and associated data. Information Processing and Management, 43(5), 1383-1401. https://doi.org/10.1016/j.ipm.2006.10.012

Kim, Y., \& Yoon, A. (2017). Scientists' data reuse behaviors: A multilevel analysis. Journal of the Association for Information Science and Technology, 68(12), 2709-2719. https://doi.org/10.1002/asi.23892

Kim, Y., \& Zhang, P. (2015). Understanding data sharing behaviors of STEM researchers: The roles of attitudes, norms, and data repositories. Library \& Information Science Research, 37(3), 189-200. https://doi.org/10.1016/j.lisr.2015.04.006

Kintsch, W., \& Van Dijk, T. A. (1978). Toward a model of text comprehension and production. Psychological Review, 85(5), 363.

Klein, G. A., Orasanu, J., Calderwood, R., Zsambok, C. E., \& others. (1993). Decision making in action: Models and methods. Ablex. 
Klein, G., Moon, B. M., \& Hoffman, R. R. (2006). Making sense of sensemaking 1: Alternative perspectives. IEEE Intelligent Systems, 21(4), 70-73. https://doi.org/10.1109/MIS.2006.75

Klein, G., Phillips, J. K., Rall, E. L., \& Peluso, D. A. (2007). A data-frame theory of sensemaking. In R. R. Hoffman (Ed.), Expertise out of context: Proceedings of the Sixth International Conference on Naturalistic Decision Making (pp. 118-160). Lawrence Erlbaum Assoc Inc.

Kling, R., \& Star, S. L. (1997). Human centered systems in the perspective of organizational and social informatics. In T. Huang \& J. Flanigan (Eds.), Human centered systems, for the National Science Foundation (p. Chapter 5). http://hdl.handle.net/2022/1798

Knorr Cetina, K. (1999). Epistemic cultures: How the sciences make knowledge. Harvard University Press.

Koesten, L. ${ }^{*}$, Gregory, K.*, Groth, P., Simperl, E. (2020). Talking datasets - Understanding data sensemaking behaviours. International Journal of Human-Computer Studies. https://doi.org/10.1016/j.ijhcs.2020.102562. *Equal contributions

Koesten, L., Kacprzak, E., Tennison, J., \& Simperl, E. (2017). The trials and tribulations of working with structured data: A study on Information seeking behavior. Proceedings of the 2017 CHI Conference on Human Factors in Computing Systems, 1277-1289.

https://doi.org/10.1145/3025453.3025838

Koesten, L., Kacprzak, E., Tennison, J., \& Simperl, E. (2019). Collaborative practices with structured data: Do tools support what users need? Proceedings of the $2019 \mathrm{CHI}$ Conference on Human Factors in Computing Systems, CHI 2019, 100. https://doi.org/10.1145/3290605.3300330

Koesten, L., Mayr, P., Groth, P., Simperl, E., \& de Rijke, M. (2019). Report on the DATA: SEARCH'18 workshop: Searching data on the web. ACM SIGIR Forum, 52(2), 117-124. https://doi.org/10.1145/3308774.3308794

Koesten, L., Simperl, E., Blount, T., Kacprzak, E., \& Tennison, J. (2019). Everything you always wanted to know about a dataset: Studies in data summarization. International Journal of Human-Computer Studies, 135, 102367. 
Koesten, L., Vougiouklis, P., Simperl, E., \& Groth, P. (2020). Dataset reuse: Translating principles to practice. https://papers.ssrn.com/sol3/papers.cfm?abstract id=3589836

Koziol, N., A., \& Bilder, C., R. (2014a). MRCV: A package for analyzing categorical variables with multiple response options. The R Journal, 6(1), 144. https://doi.org/10.32614/RJ-2014-014

Koziol, N., A., \& Bilder, C. R. (2014b). MRCV: Methods for analyzing multiple response categorical variables (MRCVs) (0.3-3) [Computer software]. https://CRAN.Rproject.org/package $=\mathrm{MRCV}$

Kratz, J., \& Strasser, C. (2014). Data publication consensus and controversies. F1000Research, 3. https://www.ncbi.nlm.nih.gov/pmc/articles/PMC4097345/pdf/f1000research-3-5878.pdf

Kratz, J., \& Strasser, C. (2015). Making data count. Scientific Data, 2(1), 150039. https://doi.org/10.1038/sdata.2015.39

Kriesberg, A., Frank, R. D., Faniel, I. M., \& Yakel, E. (2013). The role of data reuse in the apprenticeship process. Proceedings of the American Society for Information Science and Technology, 50(1), 1-10.

Kross, S., \& Guo, P. J. (2019). Practitioners teaching data science in industry and academia: Expectations, workflows, and challenges. Proceedings of the $2019 \mathrm{CHI}$ Conference on Human Factors in Computing Systems, 263. https://doi.org/10.1145/3290605.3300493 Kuhlthau, C. (1991). Inside the search process: Information seeking from the user's perspective. Journal of the American Society for Information Science, 42(5), 361-371. https://doi.org/10.1002/(SICI)1097-4571(199106)42:5<361::AID-ASI6>3.0.CO;2-\#

Kuhlthau, C. (2004). Seeking meaning: A process approach to library and information services. Libraries Unlimited. https://books.google.at/books?id=feDgAAAAMAAJ

Kunze, S. R., \& Auer, S. (2013). Dataset retrieval. Proceedings - 2013 IEEE 7th International Conference on Semantic Computing, ICSC 2013, 1-8. https://doi.org/10.1109/ICSC.2013.12 Lamb, R., \& Kling, R. (2003). Reconceptualizing users as social actors in information systems research. MIS Quarterly, 27(2), 197-235.

Larsen, S., Hamilton, S., Lucido, J., Garner, B., \& Young, D. (2016). Supporting diverse data providers in the open water data initiative: Communicating water data quality and fitness of 
use. Journal of the American Water Resources Association, 52(4), 859-872.

\section{https://doi.org/10.1111/1752-1688.12406}

Latour, B. (1983). Give me a laboratory and I will raise the world. In K. Knorr-Cetina \& M. Mulkay (Eds.), Science observed: Perspectives on the social study of science (pp. 141-170). Sage Publications.

Lave, J., \& Wenger, E. (1991). Situated learning: Legitimate peripheral participation. Cambridge University Press.

Law, J. (1992). Notes on the theory of the actor-network: Ordering, strategy, and heterogeneity. Systems Practice, 5(4), 379-393. https://doi.org/10.1007/BF01059830

Lee, C. A. (2011). A framework for contextual information in digital collections. Journal of Documentation, 67(1), 95-143. https://doi.org/10.1108/00220411111105470

Leonelli, S. (2013). Integrating data to acquire new knowledge: Three modes of integration in plant science. Studies in History and Philosophy of Science Part C: Studies in History and Philosophy of Biological and Biomedical Sciences, 44(4, Part A), 503-514.

\section{https://doi.org/10.1016/j.shpsc.2013.03.020}

Leonelli, S. (2015). What counts as scientific data? A relational framework. Philosophy of Science, 82(5), 810-821. https://doi.org/10.1086/684083

Leonelli, S. (2016). Data-centric biology: A philosophical study. University of Chicago Press. Leonelli, S. (2019). Data governance is key to interpretation: Reconceptualizing data in data science. Harvard Data Science Review, 1(1). https://doi.org/10.1162/99608f92.17405bb6

Leonelli, S., \& Ankeny, R. A. (2015). Repertoires: How to transform a project into a research community. BioScience, 65(7), 701-708. https://doi.org/10.1093/biosci/biv061

Levallois, C., Steinmetz, S., \& Wouters, P. (2013). Sloppy data floods or precise social science methodologies?: Dilemmas in the transition to data-intensive research in sociology and economics. In Virtual knowledge: Experimenting in the humanities and the social sciences (pp. 151-182). MIT Press. 
Levin, N., \& Leonelli, S. (2017). How does one "open" science? Questions of value in biological research. Science, Technology, \& Human Values, 42(2), 280-305. https://doi.org/10.1177/0162243916672071

Ley, T., \& Seitlinger, P. (2015). Dynamics of human categorization in a collaborative tagging system: How social processes of semantic stabilization shape individual sensemaking. Computers in Human Behavior, 51, 140-151. https://doi.org/10.1016/j.chb.2015.04.053

Li, X., Schijvenaars, B. J. A., \& de Rijke, M. (2017). Investigating queries and search failures in academic search. Information Processing \& Management, 53(3), 666-683. https://doi.org/10.1016/j.ipm.2017.01.005

Li, Y., \& Belkin, N. J. (2008). A faceted approach to conceptualizing tasks in information seeking. Information Processing \& Management, 44(6), 1822-1837. https://doi.org/10.1016/j.ipm.2008.07.005

Library Carpentry. (2019). Mozilla-Library Carpentry Global Sprint, 30-31 May, 2019. https://librarycarpentry.org/blog/2019/03/lc-mozilla-global-sprint/

Lievrouw, L. A. (2001). New media and the pluralization of life-worlds': A role for information in social differentiation. New Media \& Society, 3(1), 7-28. https://doi.org/10.1177/1461444801003001002

Lievrouw, L. A., \& Farb, S. E. (2003). Information and equity. Annual Review of Information Science and Technology, 37(1), 499-540. https://doi.org/10.1002/aris.1440370112 Lillis, T. (2011). Legitimizing dialogue as textual and ideological goal in academic writing for assessment and publication. Arts and Humanities in Higher Education, 10(4), 401-432. https://doi.org/10.1177/1474022211398106

Lincoln, Y., \& Guba, E. (1985). Naturalistic inquiry. Sage Publications.

Lopez-Veyna, J. I., Sosa-Sosa, V. J., \& Lopez-Arevalo, I. (2012). KESOSD: keyword search over structured data. Proceedings of the Third International Workshop on Keyword Search on Structured Data, 23-31. 
Mahyar, N., \& Tory, M. (2014). Supporting communication and coordination in collaborative sensemaking. IEEE Transactions on Visualization and Computer Graphics, 20(12), 16331642. https://doi.org/10.1109/TVCG.2014.2346573

Maier, D., Megler, V. M., Baptista, A. M., Jaramillo, A., Seaton, C., \& Turner, P. J. (2012). Navigating oceans of data. In A. Ailamaki \& S. Bowers (Eds.), Scientific and statistical database management. SSDBM 2012. (Vol. 7338, pp. 1-19). Springer. https://doi.org/10.1007/978-3-642-31235-9 1

Maier, D., Megler, V. M., \& Tufte, K. (2014). Challenges for dataset search. In S. S. Bhowmick, C. E. Dyreson, C. S. Jensen, M. L. Lee, A. Muliantara, \& B. Thalheim (Eds.), Database Systems for Advanced Applications. DASFAA 2014. (Vol. 8421, pp. 1-15). Springer. https://doi.org/10.1007/978-3-319-05810-8 1

Maitlis, S. (2005). The social processes of organizational sensemaking. Academy of Management Journal, 48(1), 21-49.

Maitlis, S., \& Christianson, M. (2014). Sensemaking in organizations: Taking stock and moving forward. Academy of Management Annals, 8(1), 57-125.

Malakis, S., \& Kontogiannis, T. (2013). A sensemaking perspective on framing the mental picture of air traffic controllers. Applied Ergonomics, 44(2), 327-339.

Marchionini, G. (2006). Exploratory search: From finding to understanding. Communications of the ACM, 49(4), 41-46. https://doi.org/10.1145/1121949.1121979

Marchionini, G., Haas, S. W., Zhang, J., \& Elsas, J. L. (2005). Accessing government statistical information. Computer, 38(12), 52-61. https://doi.org/10.1109/MC.2005.393

Marchionini, G., \& Komlodi, A. (1998). Design of interfaces for information seeking. Annual Review of Information Science and Technology, 33, 89-130.

Marchionini, G., \& White, R. (2007). Find what you need, understand what you find. International Journal of Human Computer Interaction, 23(3), 205-237. https://doi.org/10.1080/10447310701702352

Marchionini, G., \& White, R. W. (2009). Information-seeking support systems. Computer, 42(3), 30-32. https://doi.org/10.1109/MC.2009.88 
Markonis, D, Holzer, M., Dungs, S., Vargas, A., Langs, G., Kriewel, S., \& Müller, H. (2012). A survey on visual information search behavior and requirements of radiologists. Methods of Information in Medicine, 51(6), 539-548. https://doi.org/10.3414/ME11-02-0025

Markonis, D., Schaer, R., de Herrera, A. G. S., \& Müller, H. (2017). The parallel distributed image search engine (ParaDISE). ArXiv: 1701.05596, 1-23.

Martín-Mora, E., Ellis, S., \& Page, L. M. (2020). Use of web-based species occurrence information systems by academics and government professionals. PLOS ONE, 15(7), e0236556. https://doi.org/10.1371/journal.pone.0236556

Mayernik, M. S. (2011). Metadata realities for cyberinfrastructure: Data authors as metadata creators. http://www.ssrn.com/abstract $=2042653$

Mayernik, M. S. (2016). Research data and metadata curation as institutional issues. Journal of the Association for Information Science and Technology, 67(4), 973-993. https://doi.org/10.1002/asi.23425

Mayernik, M. S., \& Acker, A. (2018). Tracing the traces: The critical role of metadata within networked communications. Journal of the Association for Information Science and Technology, 69(1), 177-180. https://doi.org/10.1002/asi.23927

Mayernik, M. S., Callaghan, S., Leigh, R., Tedds, J., \& Worley, S. (2015). Peer review of datasets: When, why, and how. Bulletin of the American Meteorological Society, 96(2), 191201. https://doi.org/10.1175/BAMS-D-13-00083.1

McDonald, J. H. (n.d.). Multiple Comparisons. In Handbook of Biological Statistics (3rd ed.). Sparky House Publishing. http://www.biostathandbook.com/multiplecomparisons.html McNie, E. C. (2007). Reconciling the supply of scientific information with user demands: An analysis of the problem and review of the literature. Environmental Science and Policy, 10(1), 17-38. https://doi.org/10.1016/j.envsci.2006.10.004

Megler, V. M., \& Maier, D. (2012). When big data leads to lost data. Proceedings of the 5th Ph.D. Workshop on Information and Knowledge, 1-8. https://doi.org/10.1145/2389686.2389688 
Megler, V. M., \& Maier, D. (2013). Data near here: Bringing relevant data closer to scientists. Computing in Science and Engineering, 15(3), 44-53.

\section{https://doi.org/10.1109/MCSE.2013.38}

Meyer, E. T. (2014). Examining the hyphen: The value of social informatics for research and teaching. In P. Fichman \& F. Rosenbaum (Eds.), Social informatics: Past, present and future (pp. 56-72). Cambridge Scholarly Publishers. http://ssrn.com/

Meyer, E. T., Bulger, M., Kyriakidou-Zacharoudiou, A., Power, L., Williams, P., Venters, W., Terras, M., \& Wyatt, S. (2011). Collaborative yet independent: Information practices in the physical sciences. Research Information Network.

Michener, W. K. (2015). Ecological data sharing. Ecological Informatics, 29, 33-44.

\section{https://doi.org/10.1016/j.ecoinf.2015.06.010}

Miller, J. R., Groom, M., Hess, G. R., Steelman, T., Stokes, D. L., Thompson, J., Bowman, T., Fricke, L., King, B., \& Marquardt, R. (2009). Biodiversity conservation in local planning. Conservation Biology, 23(1), 53-63.

Mongeon, P., \& Paul-Hus, A. (2016). The journal coverage of Web of Science and Scopus: A comparative analysis. Scientometrics, 106(1), 213-228. https://doi.org/10.1007/s11192-015$\underline{1765-5}$

Mons, B., Neylon, C., Velterop, J., Dumontier, M., da Silva Santos, L. O. B., \& Wilkinson, M. D. (2017). Cloudy, increasingly FAIR; revisiting the FAIR Data Guiding Principles for the European Open Science Cloud. Information Services \& Use, 37(1), 49-56.

\section{https://doi.org/10.3233/ISU-170824}

Müller, H., Despont-Gros, C., Hersh, W., Jensen, J., Lovisa, C., \& Antoine Geissbuhler. (2006). Health care professionals' image use and search behavior. Proceedings of the Medical Informatics Europe Conference (MIE 2006), 24-32.

Muller, M. J., Lange, I., Wang, D., Piorkowski, D., Tsay, J., Liao, Q. V., Dugan, C., \& Erickson, T. (2019). How data science workers work with data: Discovery, capture, curation, design, creation. Proceedings of the 2019 CHI Conference on Human Factors in Computing Systems, CHI 2019, 126. https://doi.org/10.1145/3290605.3300356 
Murillo, A. P. (2015). Examining data sharing and data reuse in the DataONE environment. Proceedings of the Association for Information Science and Technology, 51(1), 1-5. https://doi.org/10.1002/meet.2014.14505101155

Mutschke, P., Mayr, P., \& Scharnhorst, A. (2004). Editorial for the Proceedings of the Workshop Knowledge Maps and Information Retrieval (KMIR2014) at Digital Libraries 2014. In P. Mutschke, P. Mayr, \& A. Scharnhorst (Eds.), KMIR 2014—Knowledge Maps and Information Retrieval: Proceedings of the First Workshop on Knowledge Maps and Information Retrieval (Vol. 1311, p. 6). CEUR-WS.org. http://ceur-ws.org/Vol-1311/

Nardi, B. A., \& O’Day, V. (1999). Information ecologies: Using technology with heart. MIT Press. National Science Board. (2005). Long-lived digital data collections: Enabling research and education in the 21st century (No. NSB0540). National Science Foundation. https://www.nsf.gov/pubs/2005/nsb0540/nsb0540.pdf

National Science Foundation. (2007). Cyberinfrastructure vision for 21st century discovery. https://www.nsf.gov/pubs/2007/nsf0728/nsf0728.pdf

National Science Foundation. (2020). Harnessing the Data Revolution: Coordination Hub (HDR Central) | NSF - National Science Foundation.

https://www.nsf.gov/funding/pgm summ.jsp?pims id=505820

Neff, G., Tanweer, A., Fiore-Gartland, B., \& Osburn, L. (2017). Critique and contribute: A practicebased framework for improving critical data studies and data science. Big Data, 5(2), 85-97. https://doi.org/10.1089/big.2016.0050

Niccolucci, F., \& Richards, J. D. (2013). ARIADNE: Advanced Research Infrastructures for Archaeological Dataset Networking in Europe. International Journal of Humanities and Arts Computing, 7(1-2), 70-88. https://doi.org/10.3366/ijhac.2013.0082

Noorman, M., Wessel, B., Sveinsdottir, T., \& Wyatt, S. (2018). Understanding the 'open' in making research data open: Policy rhetoric and research practice. In A. R. Saetan, I. Schneider, \& N. Green (Eds.), The politics and policies of big data: Big data big brother (pp. 292-318). Taylor \& Francis (Routledge). 
Norman, D. A., \& Draper, S. W. (1986). User centered system design; New perspectives on human-computer interaction. L. Erlbaum Associates Inc.

Noy, N., \& Benjelloun, O. (2020, August 25). An analysis of online datasets using Dataset Search (Published, in Part, as a Dataset). Google Al Blog. http://ai.googleblog.com/2020/08/ananalysis-of-online-datasets-using.html

Noy, N., Burgess, M., \& Brickley, D. (2019). Google Dataset Search: Building a search engine for datasets in an open Web ecosystem. The World Wide Web Conference- WWW '19, 13651375. https://doi.org/10.1145/3308558.3313685

Ohno-Machado, L., Sansone, S., Alter, G., Fore, I., Grethe, J., Xu, H., Gonzalez-beltran, A., Rocca-serra, P., Gururaj, A. E., Bell, E., Soysal, E., Zong, N., \& Kim, H. (2017). Finding useful data across multiple biomedical data repositories using DataMed. Nature Genetics, 49(6), 4-7.

Oudshoorn, N. E.J., \& Pinch, T. (2003). Introduction: How users and non-users matter. In Oudshoorn, N.E.J. \& Pinch, T. (Eds.), How users matter. The co-construction of users and technology (pp. 1-25). MIT Press. https://research.utwente.nl/en/publications/introductionhow-users-and-nonusers-matter(21c93d5a-f51b-47dd-a388-78583c2f8904).html

Pallickara, S. L., Pallickara, S., \& Zupanski, M. (2012). Towards efficient data search and subsetting of large-scale atmospheric datasets. Future Generation Computer Systems, 28(1), 112-118. https://doi.org/10.1016/j.future.2011.05.010

Pallickara, S. L., Pallickara, S., Zupanski, M., \& Sullivan, S. (2010). Efficient metadata generation to enable interactive data discovery over large-scale scientific data collections. Proceedings of the 2010 IEEE Second International Conference on Cloud Computing Technology and Science, 573-580. https://doi.org/10.1109/CloudCom.2010.99

Palmer, C. L. (1999). Structures and strategies of interdisciplinary science. Journal of the American Society for Information Science, 50(3), 242-253. https://doi.org/10.1002/(SICI)1097-4571(1999)50:3<242::AID-ASI7>3.0.CO;2-7 
Palmer, C. L., Cragin, M. H., \& Hogan, T. P. (2004). Information at the intersections of discovery: Case studies in neuroscience. Proceedings of the American Society for Information Science and Technology, 41, 448-455. https://doi.org/10.1002/meet.1450410152

Park, H., \& Wolfram, D. (2017). An examination of research data sharing and re-use: Implications for data citation practice. Scientometrics, 111(1), 443-461. https://doi.org/10.1007/s11192$\underline{017-2240-2}$

Park, H., You, S., \& Wolfram, D. (2018). Informal data citation for data sharing and reuse is more common than formal data citation in biomedical fields. Journal of the Association for Information Science and Technology, 69(11), 1346-1354. https://doi.org/10.1002/asi.24049

Park, Y. M., Squizzato, S., Buso, N., Gur, T., \& Lopez, R. (2017). The EBI search engine: EBI search as a service-Making biological data accessible for all. Nucleic Acids Research, 45(W1), W545-W549. https://doi.org/10.1093/nar/gkx359

Parry, O., \& Mauthner, N. (2005). Back to basics: Who re-uses qualitative data and why? Sociology, 39(2), 337-342. https://doi.org/10.1177/0038038505050543

Parsons, M. A. (2011). Making data useful for modelers to understand complex earth systems. Earth Science Informatics, 4(4), 197-223. https://doi.org/10.1007/s12145-011-0089-0

Pasquetto, I. V., Borgman, C. L., \& Wofford, M. F. (2019). Uses and reuses of scientific data: The data creators' advantage. Harvard Data Science Review, 1(2). https://doi.org/10.1162/99608f92.fc14bf2d

Pasquetto, I. V., Randles, B. M., \& Borgman, C. L. (2017). On the reuse of scientific data. Data Science Journal, 16(8), 1-9. https://doi.org/10.5334/dsj-2017-008

Passi, S., \& Jackson, S. J. (2018). Trust in data science: Collaboration, translation, and accountability in corporate data science projects. Proceedings of the ACM on HumanComputer Interaction, 2(CSCW), 136. https://doi.org/10.1145/3274405

Pepe, A., Goodman, A., Muench, A., Crosas, M., \& Erdmann, C. (2014). How do astronomers share data? Reliability and persistence of datasets linked in AAS publications and a qualitative study of data practices among US astronomers. PLOS ONE, 9(8). https://doi.org/10.1371/journal.pone.0104798 
Peters, B. G. (2017). What is so wicked about wicked problems? A conceptual analysis and a research program. Policy and Society, 36(3), 385-396.

\section{https://doi.org/10.1080/14494035.2017.1361633}

Pettigrew, K. E. (1999). Waiting for chiropody: Contextual results from an ethnographic study of the information behavior among attendees at community clinics. Information Processing \& Management, 35(6), 801-817. https://doi.org/10.1016/S0306-4573(99)00027-8

Pigott, T. D. (2001). A review of methods for missing data. Educational Research and Evaluation, 7(4), 353-383.

Pinch, T. J., \& Bijker, W. E. (1984). The social construction of facts and artefacts: Or how the sociology of science and the sociology of technology might benefit each other. Social Studies of Science, 14(3), 399-441. https://doi.org/10.1177/030631284014003004

Pirolli, P., \& Card, S. (2005). The sensemaking process and leverage points for analyst technology as identified through cognitive task analysis. Proceedings of International Conference on Intelligence Analysis, 5, 2-4.

Poth, C. N. (2019). Rigorous and ethical qualitative data reuse: Potential perils and promising practices. International Journal of Qualitative Methods, 18, 1609406919868870. https://doi.org/10.1177/1609406919868870

Pröll, S., \& Rauber, A. (2013). Scalable data citation in dynamic, large databases: Model and reference implementation. 2013 IEEE International Conference on Big Data, 307-312. https://doi.org/10.1109/BigData.2013.6691588

Pullin, A., Knight, T., Stone, D., \& Charman, K. (2004). Do conservation managers use scientific evidence to support their decision making? Biological Conservation, 119(2), 245-252.

R Core Team. (2017). R: A language and environment for statistical computing. https://www.rproject.org

Research Data Alliance. (n.d.). RDA Data Discovery Paradigms Interest Group. Retrieved October 14, 2020, from https://www.rd-alliance.org/groups/data-discovery-paradigms-ig

Ribes, D., Hoffman, A. S., Slota, S. C., \& Bowker, G. C. (2019). The logic of domains. Social Studies of Science, 49(3), 281-309. https://doi.org/10.1177/0306312719849709 
Rieh, S. Y., Collins-Thompson, K., Hansen, P., \& Lee, H.-J. (2016). Towards searching as a learning process: A review of current perspectives and future directions. Journal of Information Science, 42(1), 19-34. https://doi.org/10.1177/0165551515615841

Rieh, S. Y., \& Xie, H. (2006). Analysis of multiple query reformulations on the web: The interactive information retrieval context. Information Processing and Management, 42(3), 751-768. https://doi.org/10.1016/j.ipm.2005.05.005

Robinson-García, N., Jiménez-Contreras, E., \& Torres-Salinas, D. (2016). Analyzing data citation practices using the data citation index. Journal of the Association for Information Science and Technology, 67(12), 2964-2975. https://doi.org/10.1002/asi.23529

Robson, C., \& McCartan, K. (2016). Real world research. John Wiley \& Sons.

Rogers, Y., Sharp, H., \& Preece, J. (2012). Interaction design: Beyond human-computer interaction (3rd ed.). Wiley. http://eu.wiley.com/WileyCDA/WileyTitle/productCdEHEP001645.html

Rolland, B., \& Lee, C. P. (2013). Beyond trust and reliability: Reusing data in collaborative cancer epidemiology research. In A. Bruckman, S. Counts, C. Lampe, \& L. G. Terveen (Eds.), Computer Supported Cooperative Work, CSCW 2013 (pp. 435-444). ACM. https://doi.org/10.1145/2441776.2441826

Rudis, B., Ross, N., \& Garnier, S. (2018). The viridis color palettes. https://cran.rproject.org/web/packages/viridis/vignettes/intro-to-viridis.html

Russell, D. M. (2003). Learning to see, seeing to learn: Visual aspects of sensemaking. Human Vision and Electronic Imaging VIII, 8-21. https://doi.org/10.1117/12.501132

Russell, D. M., Stefik, M. J., Pirolli, P., \& Card, S. K. (1993). The cost structure of sensemaking. Proceedings of the SIGCHI Conference on Human Factors in Computing Systems - CHI '93, 269-276. https://doi.org/10.1145/169059.169209

Ruthven, I. (2008). Interactive information retrieval. Annual Review of Information Science and Technology, 42(1), 43-91.

Sanderson, M., \& Croft, W. B. (2012). The history of information retrieval research. Proceedings of the IEEE, 100, 1444-1451. https://doi.org/10.1109/JPROC.2012.2189916 
Sands, A., Borgman, C. L., Wynholds, L., \& Traweek, S. (2012). Follow the data: How astronomers use and reuse data. Proceedings of the American Society for Information Science and Technology, 49, 1-3. https://doi.org/10.1002/meet.14504901341

Sansone, S. A., Gonzalez-Beltran, A., Rocca-Serra, P., Alter, G., Grethe, J. S., Xu, H., Fore, I. M., Lyle, J., Gururaj, A. E., Chen, X., Kim, H. E., Zong, N., Li, Y., Liu, R., Ozyurt, I. B., \& Ohno-Machado, L. (2017). DATS, the Data Tag Suite to enable discoverability of datasets. Scientific Data, 4, 1-8. https://doi.org/10.1038/sdata.2017.59

Saracevic, T. (1996). Modeling interaction in information retrieval (IR): A review and proposal. Proceedings of the 59th Annual Meeting of the American Society for Information Science, 39.

Saracevic, T. (1997). The stratified model of information retrieval interaction: Extension and applications. Proceedings of the 60th Annual Meeting of the American Society for Information Science, 313-327.

Savolainen, R. (1995). Everyday life information seeking: Approaching information seeking in the context of "way of life." Library \& Information Science Research, 17(3), 259-294. https://doi.org/10.1016/0740-8188(95)90048-9

Savolainen, R. (2007). Information behavior and information practice: Reviewing the "umbrella concepts" of information-seeking studies. The Library Quarterly, 77(2), 109-132. https://doi.org/10.1086/517840

Savolainen, R. (2018). Information-seeking processes as temporal developments: Comparison of stage-based and cyclic approaches. Journal of the Association for Information Science and Technology. https://doi.org/10.1002/jasist.24003

Scerri, A., Kuriakose, J., Deshmane, A. A., Stanger, M., Cotroneo, P., Moore, R., Raj Naik, \& de Waard, A. (2017). Elsevier's approach to the bioCADDIE 2016 Dataset Retrieval Challenge. Database, 1-12. https://doi.org/10.1093/tropej/fmw080

Scharnhorst, A. (2015). Walking through a library remotely. Why we need maps for collections and how KnoweScape can help us to make them. Les Cahiers Du Numérique, 11(1), 103127. https://doi.org/10.3166/lcn.11.1.103-127 
Schatzki, T. R. (2001). Introduction: Practice theory. In T. R. Schatzki, K. Knorr Cetina, \& E. von Savigny (Eds.), The practice turn in contemporary theory (pp. 10-23). Routledge.

Schatzki, T. R., Knorr Cetina, K., \& Savigny, E. von (Eds.). (2001). The Practice Turn in Contemporary Theory. Routledge.

Schloss Dagstuhl - Leibniz Center for Informatics. (2018). Seminar homepage: Implementing FAIR data infrastructures.

https://www.dagstuhl.de/no cache/en/program/calendar/semhp/?semnr=18472

Schmidt, B., Gemeinholzer, B., \& Treloar, A. (2016). Open data in global environmental research: The Belmont Forum's open data survey. PLOS ONE, 11(1).

https://doi.org/10.1371/journal.pone.0146695

Schöpfel, J., Farace, D., Prost, H., \& Zane, A. (2019). Data papers as a new form of knowledge organization in the field of research data. Knowledge Organization, 46(8), 622-638. https://doi.org/10.5771/0943-7444-2019-8-622

Sedghi, S, Sanderson, M., \& Clough, P. (2011). Medical image resources used by health care professionals. Aslib Proceedings, 63(6), 570-585. https://doi.org/10.1108/00012531111187225

Sedghi, S., Sanderson, M., \& Clough, P. (2012). How do health care professionals select medical images they need? Aslib Proceedings, 64(4), 437-456. https://doi.org/10.1108/00012531211244815

Shen, Y. (2007). Information seeking in academic research: A study of the sociology faculty at the university of Wisconsin-Madison. Information Technology and Libraries, 26(1), 4-13.

Shneiderman, B. (1996). The eyes have it: A task by data type taxonomy for information visualizations. Proceedings of the 1996 IEEE Symposium on Visual Languages, 336-343. https://doi.org/10.1109/VL.1996.545307

Shove, E., Pantzar, M., \& Watson, M. (2012). The dynamics of social practice: Everyday life and how it changes. Sage. 
Shove, E., Watson, M., \& Spurling, N. (2015). Conceptualizing connections: Energy demand, infrastructures and social practices. European Journal of Social Theory, 18(3), 274-287. https://doi.org/10.1177/1368431015579964

Siebes, R., Coen, G., Gregory, K., \& Scharnhorst, A. (2019). Linked Open Data-10 Things to reach the realm of LOD (Top 10 FAIR Data and Software Things). https://zenodo.org/record/3471806

Silvello, G. (2018). Theory and practice of data citation. Journal of the Association for Information Science and Technology, 69(1), 6-20. https://doi.org/10.1002/asi.23917

Sismondo, S. (2004). An introduction to science and technology studies (1st ed.). Blackwell Publishing Ltd.

Stasko, J. T., Görg, C., \& Liu, Z. (2008). Jigsaw: Supporting investigative analysis through interactive visualization. Information Visualization, 7(2), 118-132. https://doi.org/10.1057/palgrave.ivs.9500180

Stempfhuber, M., \& Zapilko, B. (2009). Integrated retrieval of research data and publications in digital libraries. 13th International Conference on Electronic Publishing, ELPUB 2009, 613620. http://www.scopus.com/inward/record.url?eid=2-s2.0$\underline{84869140181 \& \text { partnerlD }=40 \& \text { md5=94db97ee2ae263a102066c8537823a95 }}$

Stock, W. G., \& Stock, M. (2013). Handbook of information science. De Gruyter Saur.

Straus, A., \& Corbin, J. (1990). Basics of qualitative research: Grounded theory procedures and techniques. Newbury Park, CA: Sage.

Sutcliffe, A. G., \& Ennis, M. (1998). Towards a cognitive theory of information retrieval. Interacting with Computers, 10(3), 321-351. https://doi.org/10.1016/S0953-5438(98)00013-7

Swan, A., \& Brown, S. (2008). To share or not to share: Publication and quality assurance of research data outputs (pp. 56-56). http://www.rin.ac.uk/system/files/attachments/To-sharedata-outputs-report.pdf

Tabak, E. (2014). Jumping between context and users: A difficulty in tracing information practices. Journal of the Association for Information Science and Technology, 10. 
Talja, S., \& Hansen, P. (2006). Information sharing. In A. Spink \& C. Cole (Eds.), New directions in human information behavior (pp. 113-134). Springer Netherlands.

https://doi.org/10.1007/1-4020-3670-1 7

Talja, S., Keso, H., \& Pietiläinen, T. (1999). The production of 'context' in information seeking research: A metatheoretical view. Information Processing \& Management, 35(6), 751-763. https://doi.org/10.1016/S0306-4573(99)00024-2

Tang, R., Mehra, B., Du, J. T., \& Zhao, Y. (Chris). (2019). Paradigm shift in information research. Proceedings of the Association for Information Science and Technology, 56(1), 578-581. https://doi.org/10.1002/pra2.96

Tenopir, C., Christian, L., Allard, S., \& Borycz, J. (2018). Research data sharing: Practices and attitudes of geophysicists. Earth and Space Science. https://doi.org/10.1029/2018EA000461

Tenopir, C., Allard, S., Douglass, K., Aydinoglu, A. U., Wu, L., Read, E., Manoff, M., \& Frame, M. (2011). Data sharing by scientists: Practices and perceptions. PLOS ONE, 6(6), e21101e21101. https://doi.org/10.1371/journal.pone.0021101

Tenopir, C., Dalton, E. D., Allard, S., Frame, M., Pjesivac, I., Birch, B., Pollock, D., \& Dorsett, K. (2015). Changes in data sharing and data reuse practices and perceptions among scientists worldwide. PLOS ONE, 10(8), e0134826. https://doi.org/10.1371/journal.pone.0134826

Thomas, D. R. (2006). A general inductive approach for analyzing qualitative evaluation data. American Journal of Evaluation, 27(2), 237-246. https://doi.org/10.1177/1098214005283748

Tsoukala, V., Angelaki, M., Kalaitzi, V., Wessels, B., Price, L., Taylor, M. J., Smallwood, R., Linde, P., Sondervan, J., Reilly, S., Noorman, M., Wyatt, S., Bigagli, L., Finn, R., Sveinsdottir, T., \& Wadhwa, K. (2016). RECODE: Policy recommendations for open access to research data. https://doi.org/10.5281/zenodo.50863

Tuominen, K., Talja, S., \& Savolainen, R. (2005). The social constructionist viewpoint on information practices. In K. Fisher, S. Erdelez, \& L. McKechnie (Eds.), Theories of Information Behavior (pp. 328-333). Information Today.

UK Data Service. (2019). Research data lifecycle. https://www.ukdataservice.ac.uk/managedata/lifecycle.aspx 
Ünal, Y., Chowdhury, G., Kurbanoğlu, S., Boustany, J., \& Walton, G. (2019). Research data management and data sharing behavior of university researchers. Proceedings of ISIC, 24(1), paper isic1818. http://www.informationr.net/ir/24-1/isic2018/isic1818.html

United Nations. (n.d.). Big Data for sustainable development. Retrieved July 20, 2020, from https://www.un.org/en/sections/issues-depth/big-data-sustainable-development/

van de Sandt, S., Dallmeier-Tiessen, S., Lavasa, A., \& Petras, V. (2019). The definition of reuse. Data Science Journal, 18(22), 1-19. https://doi.org/10.5334/dsj-2019-022

Van Eck, N. J., \& Waltman, L. (2010). Software survey: VOSviewer, a computer program for bibliometric mapping. Scientometrics, 84(2), 523-538.

Van Horn, J. D., \& Gazzaniga, M. S. (2013). Why share data? Lessons learned from the fMRIDC. Neurolmage, 82, 677-682. https://doi.org/10.1016/j.neuroimage.2012.11.010

Van House, N. (2004). Science and Technology Studies and Information Studies. Annual Review of Information Science and Technology, 38, 3-86.

Van House, N. A., Butler, M. H., \& Schiff, L. R. (1998). Cooperative knowledge work and practices of trust: Sharing environmental planning data sets. Proceedings of the 1998 ACM Conference on Computer Supported Cooperative Work.

Vander Sande, M., Verborgh, R., Hochstenbach, P., \& van de Sompel, H. (2018). Toward sustainable publishing and querying of distributed Linked Data archives. Journal of Documentation, 74(1), 195-222. https://doi.org/10.1108/JD-03-2017-0040

Vera-Baceta, M.-A., Thelwall, M., \& Kousha, K. (2019). Web of Science and Scopus language coverage. Scientometrics, 121(3), 1803-1813. https://doi.org/10.1007/s11192-019-03264-z

Wallis, J.C., Borgman, C. L., Mayernik, M. S., Pepe, A., Ramanathan, N. c, \& Hansen, M. (2007). Know thy sensor: Trust, data quality, and data integrity in scientific digital libraries. In L. Kovács, N. Fuhr, \& C. Meghini (Eds.), Research and Advanced Technology for Digital Libraries. ECDL 2007. Lecture Notes in Computer Science (Vol. 4675, pp. 380-391). Springer, Berlin, Heidelberg. https://doi.org/10.1007/978-3-540-74851-9 32 
Wallis, J. C., Rolando, E., \& Borgman, C. L. (2013). If we share data, will anyone use them? Data sharing and reuse in the long tail of science and technology. PLOS ONE, 8(7), e67332. https://doi.org/10.1371/journal.pone.0067332

Walshe, R., Casey, K., Kernan, J., \& Fitzpatrick, D. (2020). Introduction to the special issue on: Big data/AI standardization. Journal of ICT Standardization, 8(2).

Wang, C. L., \& Saunders, M. N. (2012). Non-response in cross-cultural surveys: Reflections on telephone survey interviews with Chinese managers. In West meets east: Toward methodological exchange (pp. 213-237). Emerald Group Publishing Limited.

Weber, N. M., Baker, K. S., Thomer, A. K., Chao, T. C., \& Palmer, C. L. (2012). Value and context in data use: Domain analysis revisited. Proceedings of the American Society for Information Science and Technology, 49. https://doi.org/10.1002/meet.14504901168

Webster, A., \& Wyatt, S. (Eds.). (2020). Health, technology and society: Critical inquiries. Palgrave Macmillan. https://doi.org/10.1007/978-981-15-4354-8

Weller, T., \& Monroe-Gulick, A. (2014). Understanding methodological and disciplinary differences in the data practices of academic researchers. Library Hi Tech, 32(3), 467-467. https://doi.org/10.1108/LHT-02-2014-0021

Wenger, E. (1999). Communities of practice: Learning, meaning, and identity. Cambridge University Press.

White, R. W. (2016). Interactions with search systems. Cambridge University Press; Cambridge Core. https://doi.org/10.1017/CB09781139525305

White, R. W., \& Roth, R. A. (2009). Exploratory search: Beyond the query-response paradigm. Morgan \& Claypool Publishers. https://doi.org/10.2200/S00174ED1V01Y200901ICR003 Whitelaw, M. (2015). Generous interfaces for digital cultural collections. Digital Humanities Quarterly, 9(1). http://www.digitalhumanities.org/dhq/vol/9/1/000205/000205.html Wilkinson, M. D., Dumontier, M., Aalbersberg, Ij. J., Appleton, G., Axton, M., Baak, A., Blomberg, N., Boiten, J.-W., da Silva Santos, L. B., Bourne, P. E., Bouwman, J., Brookes, A. J., Clark, T., Crosas, M., Dillo, I., Dumon, O., Edmunds, S., Evelo, C. T., Finkers, R., ... Mons, B. 
(2016). The FAIR Guiding Principles for scientific data management and stewardship.

Scientific Data, 3, 160018. https://doi.org/10.1038/sdata.2016.18

Williams, R., Pryor, G., Bruce, A., Macdonald, S., Marsden, W., Calvert, J., Dozier, M., \& Neilson, C. (2009). Patterns of information use and exchange: Case studies of researchers in the life sciences (pp. 56-56). Research Information Network.

Wilson, T. D. (1999). Models in information behavior research. Journal Of Documentation, 55(3), 249-270.

Wolfram, D. (2015). The symbiotic relationship between information retrieval and informetrics. Scientometrics, 102(3), 2201-2214. https://doi.org/10.1007/s11192-014-1479-0

Wu, M., Psomopoulos, F., Khalsa, S. J., \& Waard, A. de. (2019). Data discovery paradigms: User requirements and recommendations for data repositories. Data Science Journal, 18(1), 3. https://doi.org/10.5334/dsj-2019-003

Wyatt, S. (2003). Non-users also matter: The construction of users and non-users of the Internet. In Oudshoorn, N. \& Pinch, T. (Eds.), How users matter: The co-construction of users and technology (pp. 67-79). MIT Press.

https://dare.uva.nl/personal/pure/en/publications/nonusers-also-matter-the-construction-ofusers-and-nonusers-of-the-internet(9fc15327-9657-4e65-9af3-b5def673f4e9).html

Wyatt, S. (2008). Technological determinism Is dead; Long live technological determinism. In E. J. Hackett, O. Amsterdamska, M. Lynch, \& J. Wajcman (Eds.), The handbook of science \& technology studies (Vol. 7, pp. 165-181). MIT Press.

https://www.dhi.ac.uk/san/waysofbeing/data/data-crone-wyatt-2007b.pdf\#page=181

Wyatt, S., \& Balmer, B. (2007). Home on the range: What and where is the middle in science and technology studies? Science, Technology, \& Human Values, 32(6), 619-626. https://doi.org/10.1177/0162243907306085

Wyatt, S., Milojević, S., Park, H. W., \& Leydesdorff, L. (2017). Intellectual and practical contributions of scientometrics to STS. In U. Felt, R. Fouché, C. A. Miller, \& L. Smith-Doerr (Eds.), The handbook of science and technology studies (Fourth edition). The MIT Press. 
Wynholds, L., Fearon, D. S., Borgman, C. L., Traweek, S., Fearon Jr., D. S., Borgman, C. L., \& Traweek, S. (2011). When use cases are not useful: Data practices, astronomy, and digital libraries. Proceedings of the 11th Annual International ACM/IEEE Joint Conference on Digital Libraries, 383-386. https://doi.org/10.1145/1998076.1998146

Wynholds, L., Wallis, J. C., Borgman, C. L., Sands, A., \& Traweek, S. (2012). Data, data use, and scientific inquiry: Two case studies of data practices. Proceedings of the 12th ACM/IEEE-CS Joint Conference on Digital Libraries, 19-22. https://doi.org/10.1145/2232817.2232822

Xie, I. (2008). Interactive information retrieval in digital environments. IGI Publishing.

Yalçin, M. A., Elmqvist, N., \& Bederson, B. B. (2018). Keshif: Rapid and expressive tabular data exploration for novices. IEEE Transactions on Visualization and Computer Graphics, 24(8), 2339-2352. https://doi.org/10.1109/TVCG.2017.2723393

Yan, X.-S. (2011). Information science: Its past, present and future. Information, 2(3), 510-527. https://doi.org/10.3390/info2030510

Yoon, A. (2014a). "Making a square fit into a circle": Researchers' experiences reusing qualitative data. Proceedings of the American Society for Information Science and Technology, 51(1), 1-4. https://doi.org/10.1002/meet.2014.14505101140

Yoon, A. (2014b). End users' trust in data repositories: Definition and influences on trust development. Archival Science, 14(1), 17-34. https://doi.org/10.1007/s10502-013-9207-8

Yoon, A. (2016). Red flags in data: Learning from failed data reuse experiences. Proceedings of the Association for Information Science and Technology, 53, 1-6. https://doi.org/10.1002/pra2.2016.14505301126

Yoon, A. (2017a). Data reusers' trust development. Journal of the Association for Information Science and Technology, 68(4), 946-956. https://doi.org/10.1002/asi.23730

Yoon, A. (2017b). Role of communication in data reuse. Proceedings of the Association for Information Science and Technology, 463-471. 
Zhang, P., \& Soergel, D. (2014). Towards a comprehensive model of the cognitive process and mechanisms of individual sensemaking. Journal of the Association for Information Science and Technology, 65(9), 1733-1756. https://doi.org/10.1002/asi.23125

Zhang, P., \& Soergel, D. (2020). Cognitive mechanisms in sensemaking: A qualitative user study. Journal of the Association for Information Science and Technology, 71(2), 158-171. https://doi.org/10.1002/asi.24221

Zhang, S., \& Balog, K. (2018). Ad hoc table retrieval using semantic similarity. Proceedings of the 2018 World Wide Web Conference, 1553-1562. https://doi.org/10.1145/3178876.3186067

Zimmerman, A. (2003). Data sharing and secondary use of scientific data: Experiences of ecologists [The University of Michigan]. https://deepblue.lib.umich.edu/handle/2027.42/61844

Zimmerman, A. (2007). Not by metadata alone: The use of diverse forms of knowledge to locate data for reuse. International Journal on Digital Libraries, 7(1-2), 5-16.

\section{https://doi.org/10.1007/s00799-007-0015-8}

Zimmerman, A. (2008). New knowledge from old data: The role of standards in the sharing and reuse of ecological data. Science Technology and Human Values, 33(5), 631-652. https://doi.org/10.1177/0162243907306704

Zinzi, A., Capria, M. T. ,. Palomba, E. ,. Giommi, P. ,. \&. Antonelli, L. A. (2016). MATISSE: A novel tool to access, visualize and analyze data from planetary exploration missions. Astronomy and Computing, 16, 16-28. 

Summary / Samenvatting 
326 


\section{Summary}

The reuse of research data is heralded as having the potential to increase effectiveness, productivity, and reproducibility in science. Stakeholders from funders to systems designers work to create policies, repositories and tools to support and encourage opening and sharing data. An assumption underlying this work is that data will be reused if they are shared. Another assumption predicating reuse is that data will be discovered by researchers, although relatively little empirical work exists to support this assumption.

This dissertation presents empirical research about the practices involved in discovering data, examining what researchers across disciplines are actually doing as they find, make sense of and use data which they have not created. It brings together perspectives from information science (IS), computer science (CS) and science and technology studies (STS) to examine these practices with the aim of informing and intervening into the design of search solutions for research data.

As detailed in Chapter 2, user-centered models of information seeking, common in IS and CS, provide an overarching theoretical framework for this research, while concepts from STS are used to engage with these models and explore the sociotechnical nature of data discovery. A range of qualitative and quantitative methods, including semi-structured interviews, observations, a bibliometric analysis and a large-scale survey, are used throughout the thesis to draw out the complexity of practice while also identifying patterns useful for the design of data search systems.

The first empirical study, presented in Chapter 3, analytically reviews the documented data discovery practices of users of observational data across five broad disciplinary groups: astronomy, earth \& environmental sciences, biomedicine, field archeology and the social sciences. The review seeks to identify commonalities in how researchers in these disciplines discover and evaluate observational data for reuse, but it also documents the diversity of data needs and practices which exist. This chapter highlights the absence of particular communities in the reviewed literature and concludes that a theoretical framework based on information retrieval alone is insufficient for deeply understanding practices of data discovery.

Chapter 4 brings together bibliometrics and semi-structured interviews to further explore data discovery practices. A bibliometric analysis of the literature corpus collected in the third chapter reveals the technical bias and distributed nature of the discourse surrounding data 
search. Interviews with data seekers connect two different perspectives - that of data seekers and systems designers, whose input informed the development of the interview protocol. The chapter calls for a broader understanding of both the individuals seeking and using data, as well as the data needed in research. It also recognizes the liminality of practice, finding that data discovery spans the thresholds of other practices, occurring, for example during the course of data sharing and data management. It explores how adopting a contextual, sociotechnical perspective can help to understand user practices and behavior and ultimately help to improve the design of data discovery systems.

The study presented in Chapter 5 employs a broader approach - a globally distributed multidisciplinary survey, with nearly 1700 respondents - to explore data discovery practices at a larger scale. The survey questionnaire was designed to probe the role of social interactions in seeking data and to explore relations to other practices, such as searching for academic literature. An initial quantitative analysis and a more extensive exploration of the qualitative data was used to consider commonalities in practices, as well as to examine differences, looking at how data needs and search practices vary not only by disciplinary domain but also by types of data uses. The chapter suggests how data communities can be conceptualized and proposes practical applications of the findings for designers of data discovery systems and repositories.

Chapter 6, which consists of two parts, represents my first-person experience with sharing and preparing data for reuse. The main body of the chapter, published as a data descriptor paper, details the openly-available data collected from the survey described in Chapter 5. This is followed by a reflection piece, in which I consider the process of sharing and describing my data; the affordances and duplication problems data papers present; and the role of data papers in the broader landscape of scholarly communication.

The final empirical chapter, Chapter 7, focuses on a particular part of the data discovery process: data-centric sensemaking. This study, the result of an intense international collaboration, combines a think-aloud task, a screen recording analysis and in-depth interviews with researchers, as they summarized and interacted with both familiar and unfamiliar data. The chapter identifies and details common patterns of data-centric sensemaking across three clusters of activities: inspecting data, engaging with content, and placing data within broader contexts. It also explores the role of contextual information and collaborative practices in understanding data and proposes specific design recommendations to facilitate sensemaking and subsequent data reuse. 
This dissertation presents evidence of the diversity and multiplicity of data needs, discovery strategies and data uses existing both across and within disciplines. It finds that discovery practices are interwoven with other data and (re)search practices, i.e. searching for academic literature. Evidence is found for the varying ways which researchers make use of social connections - with data authors, peer networks, collaborators and broader communities - to locate and understand data. This thesis also emphasizes that discovery practices are shaped by data characteristics, as well as the hidden work involved in creating data and making them portable, i.e. processes of data description, sharing and standardization.

As a whole, this research contributes to the broader discourse on data discovery, sharing and reuse, as well as making recommendations (summarized in the final chapter) for the development of technologies which take into account the actual practices of researchers. Ultimately, this dissertation concludes that finding and reusing data are not reducible to performing keyword searches online or pressing a button to download data, demonstrating that data discovery and reuse are deeply sociotechnical practices, reliant on dynamic relationships between people, technologies, materials and policy. 


\section{Samenvatting}

\section{Vindbaar en herbruikbaar? Onderzoeksdata ontdekken in de praktijk}

Hergebruik van onderzoeksdata wordt toegejuicht als mogelijkheid om de effectiviteit, productiviteit en reproduceerbaarheid van wetenschappelijk onderzoek te vergroten.

Belanghebbenden, van financiers tot systeemontwerpers, werken samen aan regels, repositories en tools die de toegankelijkheid en beschikbaarheid van data ondersteunen en bevorderen. Een onderliggende aanname is daarbij dat gegevens worden hergebruikt als ze worden gedeeld. Een andere aanname ten gunste van hergebruik is dat de gegevens ook gevonden worden door onderzoekers, hoewel hiervoor relatief weinig empirisch bewijs bestaat.

In deze dissertatie wordt empirisch onderzoek gepresenteerd over de praktijken met betrekking tot de ontdekking van data, waarbij wordt bestudeerd wat onderzoekers in verschillende disciplines daadwerkelijk doen wanneer ze data vinden, interpreteren en gebruiken die ze niet zelf hebben gemaakt. De dissertatie combineert perspectieven uit de 'information science' (IS), 'computer science' (CS) en 'science and technology studies' (STS) om deze praktijken te onderzoeken ten behoeve van het ontwerp van zoeksystemen voor onderzoeksdata.

Zoals wordt uiteengezet in hoofdstuk 2, bieden gebruikersgerichte informatiezoekmodellen, die veel voorkomen in IS en CS, een overkoepelend theoretisch kader voor dit onderzoek, terwijl begrippen uit STS worden gebruikt om met deze modellen aan de slag te gaan en de sociaal-technische aard van data-ontdekking te verkennen. Een scala van kwalitatieve en kwantitatieve methoden, inclusief semigestructureerde interviews, observaties, een bibliometrische analyse en een grootschalige enquête, wordt in dit promotieonderzoek ingezet om de complexiteit van de praktijk aan het licht te brengen en ook om patronen aan te wijzen die bruikbaar zijn voor het ontwerp van datazoeksystemen.

In de eerste empirische studie, gepresenteerd in hoofdstuk 3, worden de gedocumenteerde data-ontdekkingspraktijken van gebruikers van observatiedata in vijf brede disciplinegroepen geanalyseerd: astronomie, aard- en milieuwetenschappen, biogeneeskunde, veldarcheologie en sociale wetenschappen. In de analyse wordt gezocht naar gemeenschappelijkheden in de manier waarop onderzoekers in deze disciplines 
observatiedata ontdekken en beoordelen met het oog op hergebruik, maar wordt ook de diversiteit van de bestaande databehoeften en -praktijken in kaart gebracht. Het hoofdstuk belicht de afwezigheid van bepaalde gemeenschappen in de onderzochte literatuur en concludeert dat een theoretisch kader dat uitsluitend gebaseerd is op informatieontsluiting ontoereikend is voor een diep inzicht in de praktijk van de data-ontdekking.

In hoofdstuk 4 worden bibliometrische data gecombineerd met semigestructureerde interviews om nader onderzoek te doen naar de praktijk van het vinden van data. Een bibliometrische analyse van de in het derde hoofdstuk verzamelde literatuur brengt de technische bias en het gedistribueerde karakter van het discours rond datazoekopdrachten aan het licht. Interviews met datazoekers leggen een link tussen twee verschillende perspectieven, dat van de zoekers en dat van de systeemontwerpers wier input heeft bijgedragen aan de ontwikkeling van het interviewprotocol. In het hoofdstuk wordt gepleit voor een breder inzicht, niet alleen in de mensen die data zoeken en gebruiken maar ook in de benodigde data zelf. Ook wordt de liminaliteit van de praktijk erkend, in die zin dat het zoeken van data de drempels naar andere praktijken overschrijdt, en bijvoorbeeld plaatsvindt tijdens datasharing en datamanagement. Verder wordt onderzocht hoe de hantering van een contextueel, sociaal-technisch perspectief tot beter begrip kan leiden voor de praktijken en het gedrag van gebruikers en uiteindelijk kan bijdragen aan een beter ontwerp van datazoeksystemen.

De in hoofdstuk 5 beschreven studie kent een bredere benadering en betreft een mondiaal verspreide multidisciplinaire enquête met bijna 1700 respondenten, bedoeld om datazoekpraktijken op grotere schaal te onderzoeken. De vragenlijst voor deze enquête is opgesteld om na te gaan welke rol sociale interacties spelen bij het zoeken van data en het verband te onderzoeken met andere praktijken, zoals het zoeken naar academische literatuur. Met een initiële kwantitatieve analyse en een meer uitgebreide verkenning van de kwalitatieve data is gekeken naar gemeenschappelijke elementen in de verschillende praktijken, maar zijn ook de verschillen onderzocht, bijvoorbeeld door te kijken hoe databehoeften en zoekpraktijken niet alleen per vakdomein maar ook per type datagebruik variëren. In het hoofdstuk worden suggesties gedaan voor de wijze waarop datagemeenschappen kunnen worden geconceptualiseerd, en worden praktische toepassingen van de uitkomsten voorgesteld voor ontwerpers van dataontdekkingssystemen en repositories. 
In hoofdstuk 6, dat uit twee delen bestaat, beschrijf ik mijn eigen ervaringen met het delen en prepareren van data voor hergebruik. De bulk van het hoofdstuk, eerder gepubliceerd als data descriptor paper, beschrijft de openbaar beschikbare data die zijn verzameld op basis van de in hoofdstuk 5 beschreven enquête. Dit deel wordt gevolgd door een reflectief deel, waarin ik inga op het proces van delen en beschrijven van mijn data, de perceptie- en duplicatieproblemen die datapapers opleveren, en de rol van datapapers in het bredere landschap van de wetenschappelijke communicatie.

Het laatste empirische hoofdstuk, hoofdstuk 7 , is gericht op een bepaald onderdeel van het proces van data-ontdekking: datacentrische betekenisgeving. In deze studie, het resultaat van intensieve internationale samenwerking, worden een aantal elementen gecombineerd: een opdracht waarbij hardop gedacht wordt, een analyse van schermopnamen en diepteinterviews met onderzoekers terwijl zij bekende en onbekende data samenvatten en gebruikten. In het hoofdstuk worden veelvoorkomende patronen van datacentrische betekenisgeving ontdekt en beschreven voor drie clusters van activiteiten: inspecteren van data, aan de slag gaan met content en plaatsen van data in bredere contexten. Ook wordt onderzocht wat de rol is van contextuele informatie en samenwerkingspraktijken bij het begrijpen van data, en worden specifieke ontwerpaanbevelingen gedaan voor de bevordering van betekenisgeving en daaropvolgend hergebruik van data.

Deze dissertatie levert bewijzen voor de bestaande diversiteit en multipliciteit van databehoeften, ontdekkingsstrategieën en datagebruik tussen en binnen disciplines. Het blijkt dat ontdekkingspraktijken verweven zijn met andere data- en (onder)zoekpraktijken, namelijk bij het zoeken naar academische literatuur. Er worden bewijzen gevonden voor de verschillende manieren waarop onderzoekers gebruikmaken van sociale verbindingen - met auteurs, peer-netwerken, samenwerkingspartners en grotere gemeenschappen - om data te lokaliseren en te interpreteren. In deze dissertatie wordt tevens benadrukt dat ontdekkingspraktijken worden gevormd door datakenmerken, en wordt aandacht besteed aan het onzichtbare werk dat verricht wordt om data te creëren en overdraagbaar te maken, dat wil zeggen de beschrijving, het delen en de standaardisatie van data.

Dit onderzoek als geheel draagt bij aan het bredere discours over het ontdekken, delen en hergebruiken van data, en heeft geleid tot aanbevelingen (samengevat in het laatste hoofdstuk) voor de ontwikkeling van technologieën die rekening houden met reële onderzoekspraktijken. Uiteindelijk is de conclusie van deze dissertatie dat het vinden en hergebruiken van data niet kan worden gereduceerd tot het uitvoeren van een onlinezoekopdracht of een druk op de knop om data te downloaden, omdat is aangetoond dat data 
vinden en data hergebruiken zeer sociaal-technische praktijken zijn die afhangen van dynamische relaties tussen mensen, technologieën, materialen en beleidsregels. 

Publications list 


\section{Publications included in the dissertation}

Koesten, L.*, Gregory, K.*, Groth, P., Simperl, E. (2020). Talking datasets: Understanding data sensemaking behaviors. International Journal of Human-Computer Studies.

https://doi.org/10.1016/.ijhcs.2020.102562. *Equal contributions.

Gregory, K. (2020). A dataset describing data discovery and reuse practices in research. Scientific Data, 7(1), 1-11.https://doi.org/10.1038/s41597-020-0569-5

Gregory, K., Groth, P. Scharnhorst, A., Wyatt, S. (2020). Lost or found? Discovering data needed for research. Harvard Data Science Review.

https://doi.org/10.1162/99608f92.e38165eb

Gregory, K., Cousijn, H., Groth, P., Scharnhorst, A., Wyatt, S. (2019). Understanding data search as a socio-technical practice. Journal of Information Science.

https://doi.org/10.1177/0165551519837182

Gregory, K., Groth, P., Cousijn, H., Scharnhorst, A., Wyatt, S. (2019). Searching data: A review of observational data retrieval practices in selected disciplines. Journal of the Association of Information Science and Technology. https://doi.org/10.1002/asi.24165

\section{Other publications produced during the $\mathrm{PhD}$}

Manola, N.; Mutschke, P.; Scherp, G.; Tochterman, K.; Wittenburg, P.; Gregory, K.;

Hasselbring, W.; den Heijer, K.; Manghi, P.; Van Uytvanck, D. (2020). Implementing FAIR data infrastructures. Dagstuhl Perspectives Workshop 18472; 1-34.

https://drops.dagstuhl.de/opus/volltexte/2020/13237/pdf/DagMan-8-1-1.pdf

Siebes, R., Coen, G., Gregory, K., Scharnhorst, A. (2019). Linked Open Data - 10 Things to reach the realm of LOD. Part of Top 10 FAIR Data and Software Things. ${ }^{32}$

http://doi.org/10.5281/zenodo.3471806

Gregory K, Khalsa S.J, Michener W.K., Psomopoulos F.E, de Waard A., Wu, M. (2018) Eleven quick tips for finding research data. PLOS Computational Biology 14(4): e1006038. https://doi.org/10.1371/journal.pcbi.1006038

\section{Datasets}

Gregory, K. (2020). Data discovery and reuse practices in research [dataset]. Data Archiving and Networked Services (DANS). https://doi.org/10.17026/dans-xsw-kkeq

${ }^{32}$ A slightly edited version of this paper is also part of a forthcoming (2020) edited book, Linking Knowledge (ERGON publisher, editors R. Smiraglia \& A. Scharnhorst). 

Acknowledgements 
This dissertation, and the four years of work leading to its creation, were only possible through the support of numerous people, whom I would like to take a moment to formally thank.

First and foremost, I would like to thank my supportive, knowledgeable and altogether wonderful supervisors, Sally Wyatt, Paul Groth and Andrea Scharnhorst. While having three supervisors, with three different areas of expertise, could have been challenging, the collaboration, camaraderie and exchange of ideas which we have shared during the past four years has been one of the highlights of my doctoral journey.

Sally, thank you for your mentorship. I greatly value the way you nudged me toward ideas and connections, which you undoubtedly saw much sooner than I did, allowing me to discover them anew. Paul, I appreciate and admire your open mindedness, straightforwardness and ability to gracefully navigate complex projects; thank you for bringing those skills to our project. Andrea, thank you for being a connector, for helping me to make connections to new ways of thinking, to new communities and within DANS.

I would like to thank the members of the dissertation committee, Wiebe Bijker, Christine Borgman, Vivien Petras and Hans Schmeets. Thank you for your time, expertise and valuable comments, which helped to improve this work. Thank you also to Francesca Morselli, Mike Priddy and Laura Koesten, the first readers of the early chapters of this thesis, and to the DANS research group who provided invaluable feedback.

Members of the Re-Search project team were also key to successfully completing my research. Thank you to all members of the team, especially Maarten de Rijke and Frank van Harmelen, for your support, interest and keen questions. Wouter Haak was also instrumental to this work. Wouter, thank you for always being encouraging, responsive and willing to do whatever possible to support my work. I would also like to thank Helena Cousijn, who was part of the Re-Search project for two years, for facilitating my research on the side of Elsevier and also for interesting conversations throughout the project.

My sincerest thanks to the Dutch Research Council (NWO) for funding my research. Thank you also to the Netherlands Graduate School of Science, Technology and Culture (WTMC), who contributed funds toward the printing of this book.

During the past four years, I have had the privilege to work with a community of intelligent and motivated individuals at DANS. I would like to thank Peter Doorn, Ingrid Dillo and Henk 
Wals for supporting my research and welcoming me to DANS, as well as the numerous DANSers who helped me to test and think through interview protocols and survey questions. Francesca Morselli, Gerry Coen, Femmy Admiraal, Ricarda Braukmann and Cees Hof thank you for the conversations, brainstorming and laughter in our co-working space. Dirk Rorda, thank you for always providing a stimulating and unique perspective. I would also like to recognize the DANS Research \& Innovation group; I value the discussions, diversity and collegiality cultivated in our meetings.

I always enjoyed and looked forward to the WTMC summer schools and workshops in Ravenstein. The close reading sessions, early morning runs, the summer school with Elizabeth Shove and the three-course vegetarian dinners are among my many fond memories. Thank you, WTMC, for helping me feel at home in the world of STS.

I have had the opportunity to engage in numerous collaborations these past years. Thank you Mingfang Wu, Fotis Psomopoulos and Siri Jodha Khalsa for our enjoyable work in the Research Data Alliance; thanks as well to Peter Mutschke and the members of the GO INTER network for helping to diversify my interests. My special thanks to Laura Koesten for engaging in truly collaborative research, in all the best senses of the word.

Writing my dissertation in the midst of the COVID-19 pandemic and various lockdowns made me grateful for the many local cafés and the beautiful library in Haarlem, as well as for the people I met there when possible, who became colleagues of a different sort. These places and people gave me needed respite from writing in my living room, as did walks and chats with my friends, especially Andrea, Tiffany and Claudia.

It is impossible to name all of the ways that my parents, Tim and Linda Brady, have supported me throughout my life and in my numerous educational ventures. Thank you both for your constant encouragement to follow my curiosity and see opportunities. My sisters, Sara and Shannon, kept me laughing and connected with life at home; thank you both. Finally, thank you to my children, Kellan and Blake. Your patience helped me to complete work when it needed to be done; your impatience reminded me that there is more to life than data and articles. Thank you for taking the first steps into learning $R$ and Python together, and thanks to my husband, Mike, for patiently coaching us on the basics of programming. Mike, thank you for being my fellow adventurer, both during this journey and all the ones to come. 

About the author 
346 
Kathleen Gregory was born in Denver, Colorado, USA on May 10, 1977. She holds a Master of Science in Library and Information Science from Drexel University (2010) and a Master of Arts in Education from the University of Colorado (2002). She has worked as an educator and academic librarian in both Germany and the United States, specializing in scholarly communication, bibliometrics and scientific information literacy. During the course of her $\mathrm{PhD}$, she was educated in science and technology studies at the Netherlands Graduate Research School of Science, Technology and Modern Culture (WTMC). 



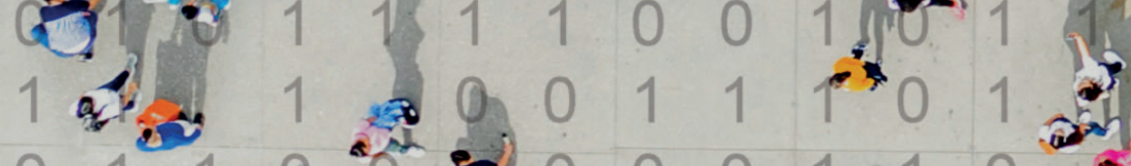

$\begin{array}{llllllllllll}0 & 1 & 1 & 0 & 0 & 3 & 0 & 0 & 0 & 1 & 1 & 0\end{array}$

$\begin{array}{lllllllllllllllll}1 & 0 & 1 & 1 & 1 & 1 & 1 & 0 & 1 & 1 & 1 & 1 & 1 & 1 & 1 & 0\end{array}$

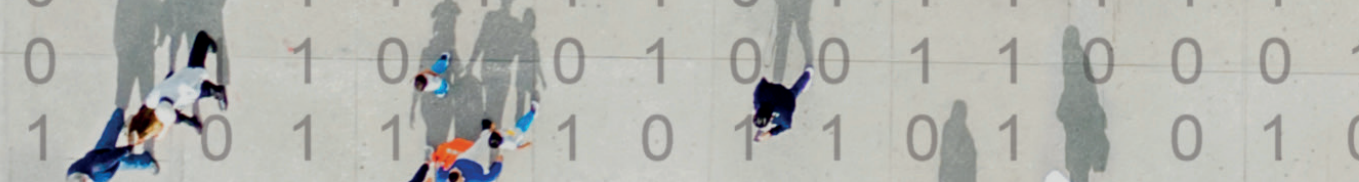

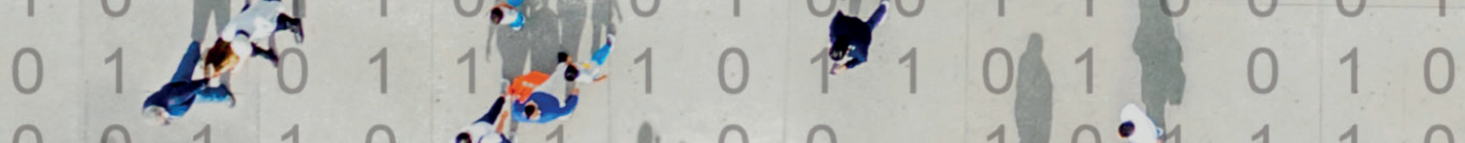

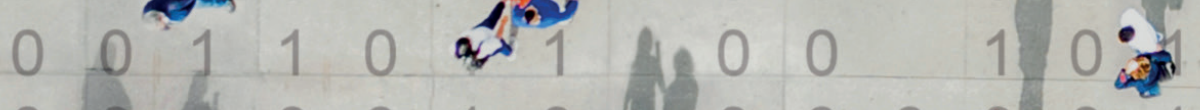

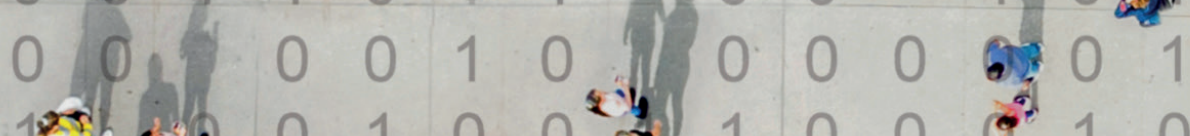

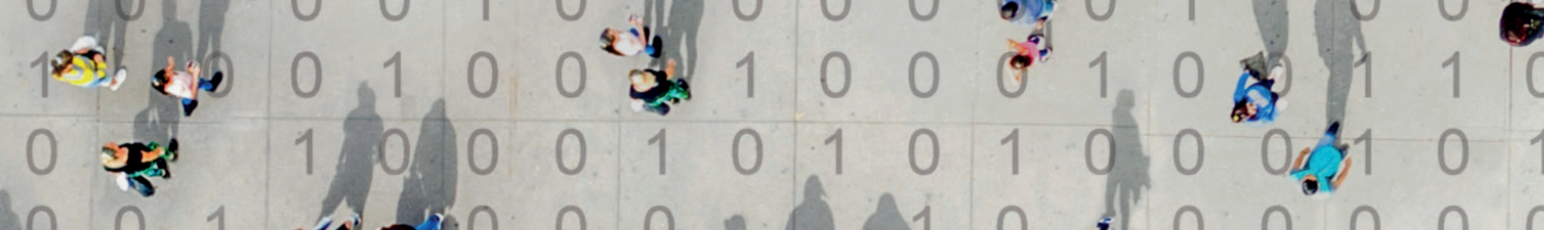

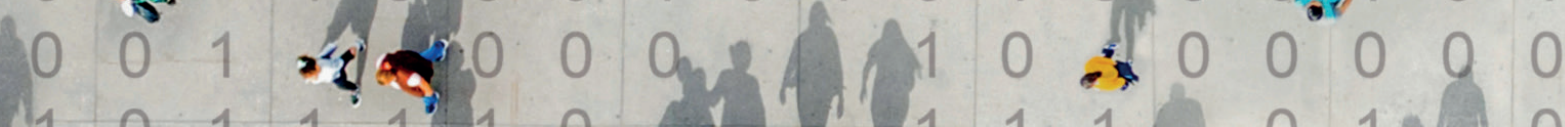

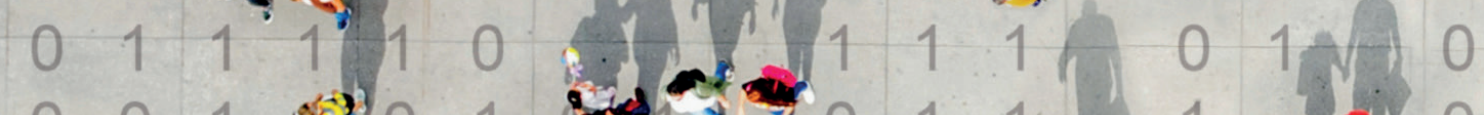

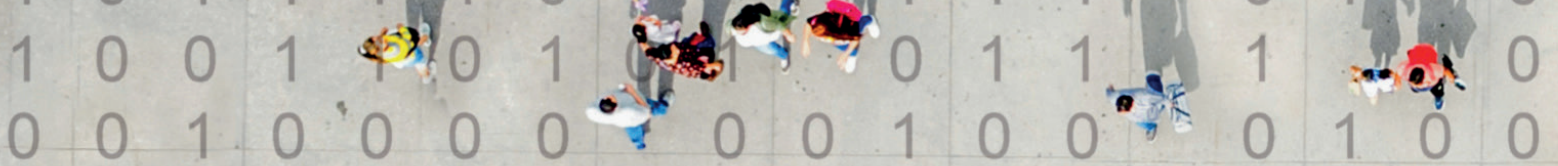
$\begin{array}{llllllllllllllllll}0 & 0 & 1 & 0 & 1 & 0 & 1 & 1 & 0 & 0 & 1 & 1 & 0 & 1 & 1 & 0 & 0\end{array}$ $\begin{array}{lllllllllllllllll}0 & 0 & 1 & 1 & 0 & 1 & 0 & 1 & 0 & 0 & 1 & 1 & 0 & 1 & 0 & 1 & 0\end{array}$ $\begin{array}{llllllllllllllll}1 & 1 & 0 & 0 & 0 & 0 & 0 & 1 & 1 & 0 & 0 & 0 & 0 & 0 & 0 & 1\end{array}$ $\begin{array}{llllllllllllllll}1 & 0 & 1 & 1 & 0 & 0 & 1 & 1 & 0 & 1 & 1 & 0 & 1 & 0 & 1 & 1\end{array}$ $\begin{array}{lllllllllllllll}0 & 0 & 0 & 0 & 1 & 1 & 0 & 0 & 0 & 0 & 0 & 1 & 1 & 0 & 0\end{array}$ $\begin{array}{lllllllllllllll}1 & 1 & 1 & 0 & 1 & 0 & 1 & 1 & 1 & 1 & 0 & 1 & 0 & 1 & 1\end{array}$ $\begin{array}{llllllllllllll}1 & 1 & 0 & 0 & 0 & 1 & 1 & 0 & 1 & 0 & 0 & 0 & 1 & 1\end{array}$ $\begin{array}{llllllllllll}1 & 1 & 1 & 0 & 1 & 1 & 1 & 1 & 1 & 0 & 1 & 1\end{array}$ $\begin{array}{lllllllll}1 & 0 & 0 & 0 & 1 & 0 & 1 & 1 & 1\end{array}$

$\begin{array}{llllll}0 & 1 & 0 & 0 & 0 & 1 \\ 1 & & 1 & 1 & 1 & 1 \\ 1 & & 0 & & 1 & 0 \\ 0 & & 0 & & 0 & \\ 0 & & 1 & & 0\end{array}$

